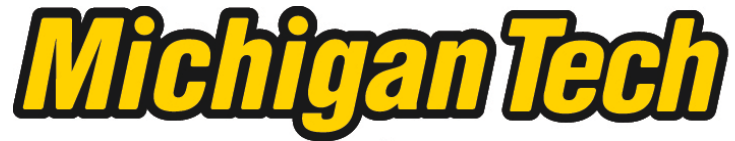 \\ Michigan Technological University Create the Future Digital Commons @ Michigan Tech
}

Dissertations, Master's Theses and Master's Reports - Open

Dissertations, Master's Theses and Master's

Reports

2007

\section{Effect of segmented anodes on the performance and plume of a hall thruster}

Alexander W. Kieckhafer

Michigan Technological University

Follow this and additional works at: https://digitalcommons.mtu.edu/etds

Part of the Mechanical Engineering Commons

Copyright 2007 Alexander W. Kieckhafer

\section{Recommended Citation}

Kieckhafer, Alexander W., "Effect of segmented anodes on the performance and plume of a hall thruster", Dissertation, Michigan Technological University, 2007.

https://doi.org/10.37099/mtu.dc.etds/375

Follow this and additional works at: https://digitalcommons.mtu.edu/etds

Part of the Mechanical Engineering Commons 


\title{
THE EFFECT OF SEGMENTED ANODES ON THE PERFORMANCE AND PLUME OF A HALL THRUSTER
}

By:

ALEXANDER W. KIECKHAFER

\author{
A DISSERTATION \\ Submitted in partial fulfillment of the requirements for the degree of: \\ DOCTOR OF PHILOSOPHY \\ (Mechanical Engineering-Engineering Mechanics) \\ MICHIGAN TECHNOLOGICAL UNIVERSITY \\ 2007
}

Copyright (C Alexander W. Kieckhafer 2007 
For my parents. 


\section{Abstract}

Development of alternative propellants for Hall thruster operation is an active area of research. Xenon is the current propellant of choice for Hall thrusters, but can be costly in large thrusters and for extended test periods. Condensible propellants may offer an alternative to xenon, as they will not require costly active pumping to remove from a test facility, and may be less expensive to purchase. A method has been developed which uses segmented electrodes in the discharge channel of a Hall thruster to divert discharge current to and from the main anode and thus control the anode temperature. By placing a propellant reservoir in the anode, the evaporation rate, and hence, mass flow of propellant can be controlled.

Segmented electrodes for thermal control of a Hall thruster represent a unique strategy of thruster design, and thus the performance of the thruster must be measured to determine the effect the electrodes have on the thruster. Furthermore, the source of any changes in thruster performance due to the adjustment of discharge current between the shims and the main anode must be characterized.

A Hall thruster was designed and constructed with segmented electrodes. It was then tested at anode voltages between 300 and $400 \mathrm{~V}$ and mass flows between 4 and $6 \mathrm{mg} / \mathrm{s}$, as well as 100\%, 75\%, 50\%, $25 \%$, and $<5 \%$ of the discharge current on the shim electrodes. The level of current on the shims was adjusted by changing the shim voltage. At each operating point, the thruster performance, plume divergence, ion energy, and multiply charged ion fraction were measured

Thruster performance exhibited a small change with the level of discharge current on the shim electrodes. Thrust and specific impulse increased by as much as 6\% and 7.7\%, respectively, as discharge current was shifted from the main anode to the shims at constant anode voltage. Thruster efficiency did not change. Plume divergence was reduced by approximately 4 degrees of half-angle at high levels of current on the shims and at all combinations of mass flow and anode voltage. The fraction of singly charged xenon in the thruster plume varied between approximately $80 \%$ and $95 \%$ as the anode voltage and mass flow were changed, but did not show a significant change with shim current. Doubly and triply charged xenon made up the remainder of the ions detected. Ion energy exhibited a mixed behavior. The highest voltage present in the thruster largely dictated the most probable energy; either shim or anode voltage, depending on which 
was higher. The overall change in most probable ion energy was $20-30 \mathrm{eV}$, the majority of which took place while the shim voltage was higher than the anode voltage. The thrust, specific impulse, plume divergence, and ion energy all indicate that the thruster is capable of a higher performance output at high levels of discharge current on the shims. The lack of a change in efficiency and fraction of multiply charged ions indicate that the thruster can be operated at any level of current on the shims without detrimental effect, and thus a condensible propellant thruster can control the anode temperature without a decrease in efficiency or a change in the multiply charged ion fraction. 


\section{Acknowledgements}

First, I'd like to acknowledge the contribution my parents made in the process of my education. They provided a fertile environment in my early years, assisted me in my schooling, and were always there if I needed assistance; moral or scholastic. I'd also like to recognize my sisters, Katherine and Margaret. I don’t say it nearly enough, but I love you all.

Next, to my advisor, Dr. King. From figuring out how to bolt a vacuum tank to the floor to the composition of this thesis, you were always available as a sounding board for my ideas, concerns, and questions. Your advice was invaluable, and I'll never forget all you did for me. I just wish we had pursued the Tungsten per square millimeter as an official unit of measure.

To my committee. You each provided a unique perspective to my thesis, and put up with the longer than anticipated process of editing and cleaning the document. Thanks for all your help.

To the ME-EM department staff: Marty, Jesse, Rob, Gerry, the staff of the main office, and anyone else who I've left out of this list. Your assistance made all those years in the basement easier. I'd like to offer a special thanks to Marty and Jesse, the machinists who tirelessly worked on all the screwy parts and materials I submitted to them. You worked miracles on a regular basis, and my work never would have been completed without it. Especially without high-purity magnet iron.

To the other students in the lab: Dean, Emily, Jason, Jason, and Jerry. You got in my way, used my tanks, my xenon, and my thrusters. But you also provided a collaborative environment that I truly will miss as I move into my life after school. And to Rob and Carrie, I regret that I didn’t get to work with you, but you have a great graduate career ahead of you. Enjoy it while it lasts, and don't leave any water lines open.

Last, but not least, to my friends. Matt, Nic, Rusty, John, Eric, and Mikey. Being able to sit around without a coherent thought in my head was invaluable, and I'll miss you all. And to all those with whom I've spoken over the past years, thanks for all the memories. It's been a blast. 


\section{Table of Contents}

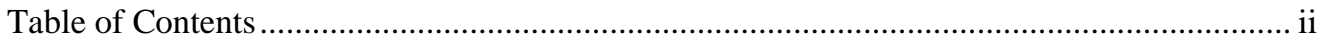

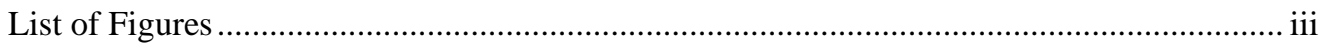

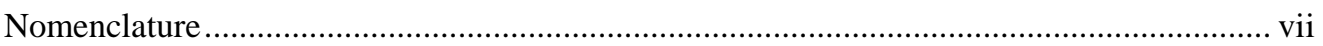

Chapter 1: $\quad$ Introduction ........................................................................................... 1

$1.1 \quad$ Motivation for Electric Propulsion........................................................................ 1

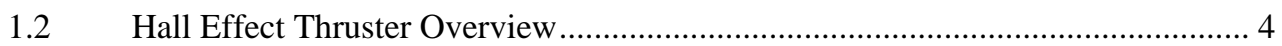

1.3 Motivation for Alternative Propellants .................................................................. 5

$1.4 \quad$ The Direct Evaporation Method............................................................................. 7

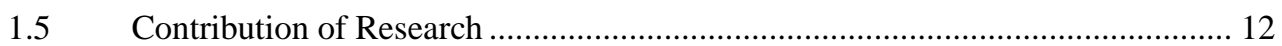

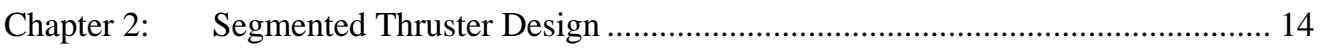

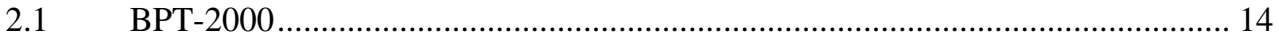

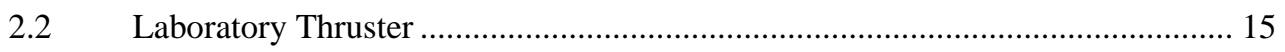

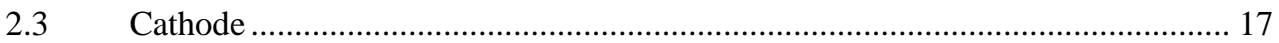

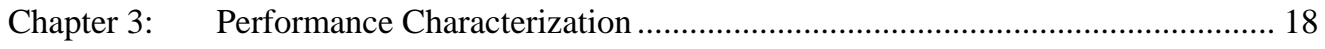

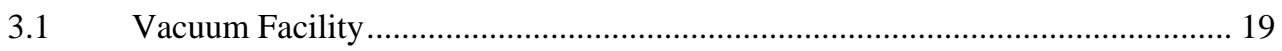

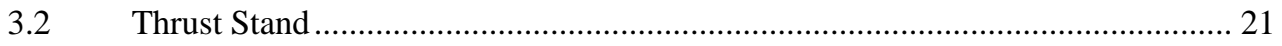

3.3 Thruster Operating Characteristics ...................................................................... 25

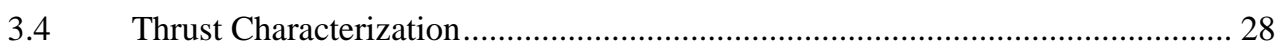

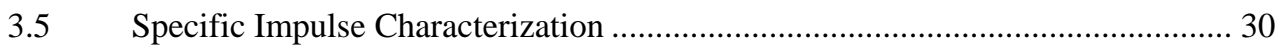

3.6 Efficiency Characterization............................................................................... 31

Chapter 4: $\quad$ Plume Divergence Characterization ................................................................... 34

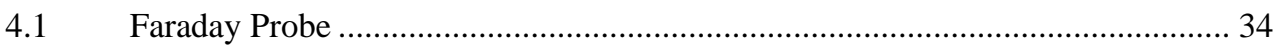

4.2 Faraday Probe Sweep Data ……….................................................................... 36

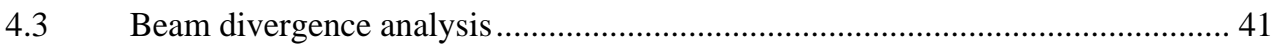

Chapter 5: $\quad$ Ion Energy Characterization............................................................................. 44

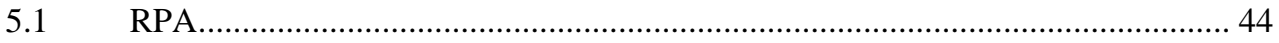




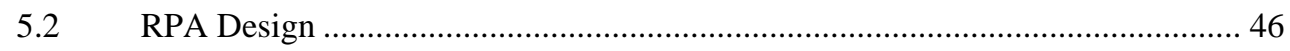

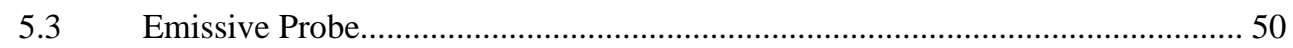

$5.4 \quad$ Determination of Plasma Potential.................................................................... 52

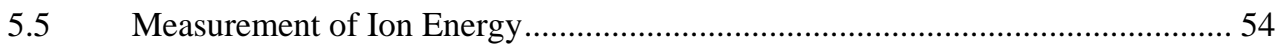

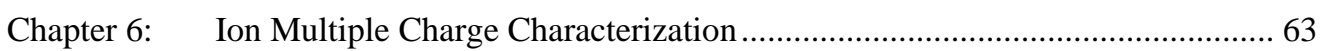

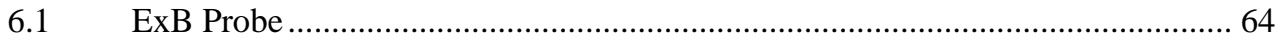

6.2 Ion Energy Error Analysis ................................................................................. 70

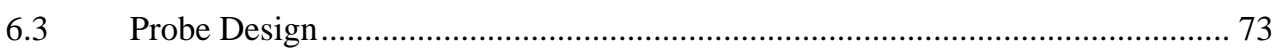

Chapter 7: Determination of the Source of Observed Thruster Performance Changes..... 96

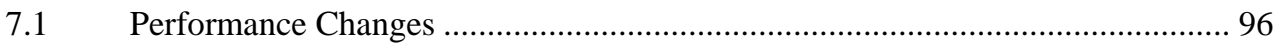

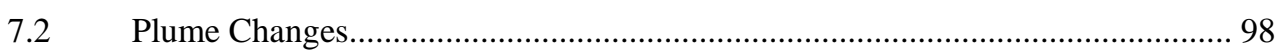

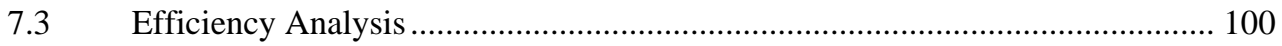

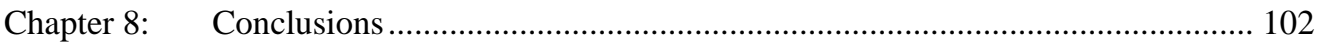

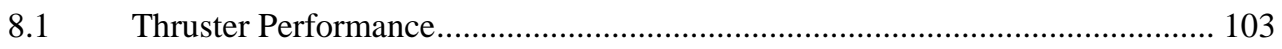

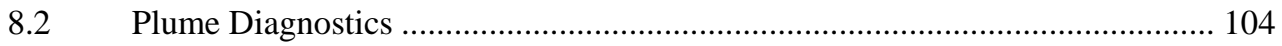

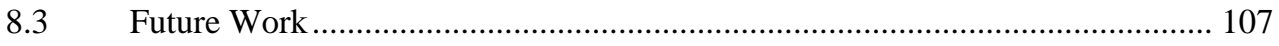

Appendix A: Motivation for Condensible Propellants ........................................................ 109

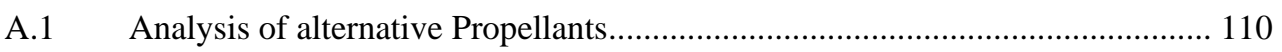

A.2 Energetics of propellant alternatives ................................................................... 111

A.2.1 Acceleration Kinetics ............................................................................... 111

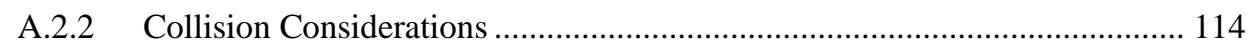

A.3 Analysis of spacecraft interactions for bismuth, xenon, and krypton ................. 118

A.4 Feed System for Condensible Propellant Operation .......................................... 121

A.5 Summary of analysis of propellant options........................................................ 121

Appendix B: Experimental and Statistical Analysis Methods........................................... 123 


\section{List of Figures}

Figure 1-1: Mass Ratios as a function of mission $\Delta \mathrm{v}$ for a chemical rocket and a Hall thruster .............. 2

Figure 1-2: Cross Sectional View of a typical Hall thruster.................................................................... 5

Figure 1-3: Cross-section view of the thruster with shim electrodes and main anode. …….................... 9

Figure 1-4: Temperature and current deposition on both the main and shim anodes as a function of time for

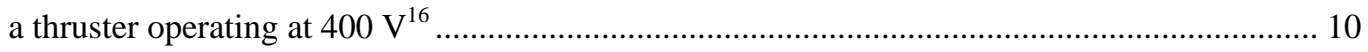

Figure 1-5: Diagram of a two-stage Hall thruster as used in Ref. 8 .................................................... 11

Figure 1-6: Observed changes in plume divergence for several segmented electrode configurations, from

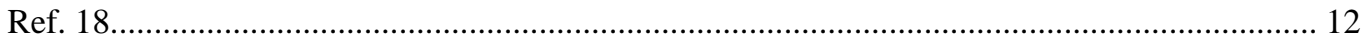

Figure 1-7: Photograph of the segmented anode thruster used in Ref. 18........................................... 12

Figure 2-1: Photograph of the BPT-2000 with cathode................................................................ 14

Figure 2-2: Cross section of the BPT-2000 Hall thruster ....................................................................... 15

Figure 2-3: Photograph of the segmented electrode hall thruster ............................................................. 16

Figure 2-4: Cross section of the segmented anode thruster ................................................................ 17

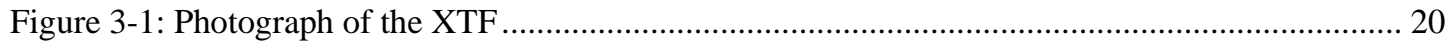

Figure 3-2: Photograph of the motion control table inside the XTF....................................................... 21

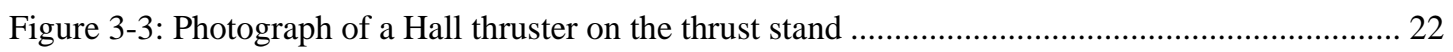

Figure 3-4: Inverted pendulum thrust stand. Green section is the movable platform which makes up the top of the inverted pendulum, violet is the tiltable supports held by leaf springs which support the movable platform, and red is the adjustable-tilt base.................................................................. 23

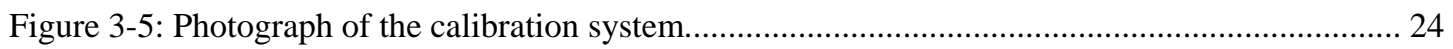

Figure 3-6: Characteristic shift in current on the shims and main anode as shim voltage was varied.

Thruster was operating at $350 \mathrm{~V}$ on the anode and $5 \mathrm{mg} / \mathrm{s}$ of mass flow...................................... 26

Figure 3-7: Shim Current as a function of shim voltage at all thruster operating points...................... 26

Figure 3-8: Total thruster current as a function of all three experimental factors ................................. 27

Figure 3-9: Discharge power as a function of all three experimental factors ....................................... 28 
Figure 3-10: Thrust measured as a function of all three experimental factors. Higher shim voltages for a given mass flow and anode voltage combination correspond to higher discharge heating acceptance by the shims. 29

Figure 3-11: Specific Impulse as a function of shim voltage for all thruster operating conditions tested. Higher shim voltage for a given anode voltage indicates higher discharge electron heating on the shims. 30

Figure 3-12: Thruster Efficiency as a function of all three experimental factors. Higher shim voltage for a given anode voltage indicates higher discharge heating on the shims 32

Figure 4-1: Photograph of the Faraday probe. 36

Figure 4-2: Beam Profiles with $300 \mathrm{~V}$ on the anode at all mass flows and levels of discharge current heating 38

Figure 4-3: Beam Profiles with $350 \mathrm{~V}$ on the anode at all mass flows and levels of discharge current heating

Figure 4-4: Beam Profile s with $400 \mathrm{~V}$ on the anode at all mass flows and levels of discharge current heating...... 40

Figure 4-5: Beam divergence as a function of all three experimental factors 42

Figure 5-1: Single-grid RPA 47

Figure 5-2: Four-grid RPA. 48

Figure 5-3: Photograph of an RPA in the vacuum facility ..... 49

Figure 5-4: Typical RPA I-V sweep and associated derivative of current with respect to voltage, with $350 \mathrm{~V}$ on the anode, $5 \mathrm{mg} / \mathrm{s}$ of propellant flow, and all discharge heating on the shims. 50

Figure 5-5: Typical emissive probe trace 52

Figure 5-6: Plasma Potential relative to ground as a function of all three experimental factors, measured on the axis of the thruster, $500 \mathrm{~mm}$ from the exit plane. 53

Figure 5-7: Derivatives of RPA current with at $4 \mathrm{mg} / \mathrm{s}$ of xenon, at all combinations of anode voltages and shim levels 56

Figure 5-8: Derivatives of RPA current with at $5 \mathrm{mg} / \mathrm{s}$ of xenon, at all combinations of anode voltages and shim levels 57 
Figure 5-9: Derivatives of RPA current with at $6 \mathrm{mg} / \mathrm{s}$ of xenon, at all combinations of anode voltages and shim levels ... 58

Figure 5-10: Most Probable Ion Energy as a function of all three experimental factors. Higher shim voltage indicates a higher proportion of discharge current on the shims.

Figure 5-11: Average ion energy as a function of all three experimental factors. Higher shim voltage for a given combination of anode voltage and mass flow indicates higher discharge heating on the shims

Figure 6-1: Major characteristics of an ExB probe 65

Figure 6-2: Typical normalized ExB probe distribution. 67

Figure 6-3: Schematic view of ExB probe showing detection of an off-axis ion. Plate C1 is the front plate of the probe, $\mathrm{C} 2$ is the filter entry or collimator exit plate, and $\mathrm{F}$ is the filter exit plate. 70

Figure 6-4: Photograph of the ExB probe with the top plate removed. 74

Figure 6-5: Resolution error of the ExB probe as a function of ion energy 75

Figure 6-6: Photograph of the ExB probe in the water-cooled shroud 76

Figure 6-7: typical set of ExB probe traces taken as the level of shim current was varied. Data are for 400

$\mathrm{V}$ on the anode and $4 \mathrm{mg} / \mathrm{s}$ of xenon. 78

Figure 6-8: ExB probe traces taken with $400 \mathrm{~V}$ on the main anode and $4 \mathrm{mg} / \mathrm{s}$ of xenon 80

Figure 6-9: Singly charged ion density fraction for all thruster operating points examined. High shim voltages indicate high amounts of discharge current attachment on the shims 81

Figure 6-10: Doubly charged ion density fraction for all thruster operating points examined. High shim voltages indicate high amounts of discharge current attachment on the shims 81

Figure 6-11: Triply charged ion density fraction for all thruster operating points examined. High shim voltages indicate high amounts of discharge current attachment on the shims 82

Figure 6-12: Average ion charge as a function of all three experimental factors. High shim voltage for indicates a higher fraction of discharge current heating on the shims. 84

Figure 6-13: ExB probe traces taken as the level of shim current was varied. Data are for $300 \mathrm{~V}$ on the anode and $4 \mathrm{mg} / \mathrm{s}$ of xenon 87 
Figure 6-14: ExB probe traces taken as the level of shim current was varied. Data are for $300 \mathrm{~V}$ on the anode and $5 \mathrm{mg} / \mathrm{s}$ of xenon

Figure 6-15: ExB probe traces taken as the level of shim current was varied. Data are for $300 \mathrm{~V}$ on the anode and $6 \mathrm{mg} / \mathrm{s}$ of xenon 89

Figure 6-16: ExB probe traces taken as the level of shim current was varied. Data are for $350 \mathrm{~V}$ on the anode and $4 \mathrm{mg} / \mathrm{s}$ of xenon 90

Figure 6-17: ExB probe traces taken as the level of shim current was varied. Data are for $350 \mathrm{~V}$ on the anode and $5 \mathrm{mg} / \mathrm{s}$ of xenon 91

Figure 6-18: ExB probe traces taken as the level of shim current was varied. Data are for $350 \mathrm{~V}$ on the anode and $6 \mathrm{mg} / \mathrm{s}$ of xenon 92

Figure 6-19: ExB probe traces taken as the level of shim current was varied. Data are for $400 \mathrm{~V}$ on the anode and $4 \mathrm{mg} / \mathrm{s}$ of xenon 93

Figure 6-20: ExB probe traces taken as the level of shim current was varied. Data are for $400 \mathrm{~V}$ on the anode and $5 \mathrm{mg} / \mathrm{s}$ of xenon 94

Figure 6-21: ExB probe traces taken as the level of shim current was varied. Data are for $400 \mathrm{~V}$ on the anode and $6 \mathrm{mg} / \mathrm{s}$ of xenon 95

Figure A-1: Change in thrust as a function of propellant ion mass, assuming constant acceleration voltage 113

Figure A-2: Fraction of thruster power required for ionization vs. $\mathrm{I}_{\mathrm{sp}}$ for all propellants examined.. 114 


\section{Nomenclature}

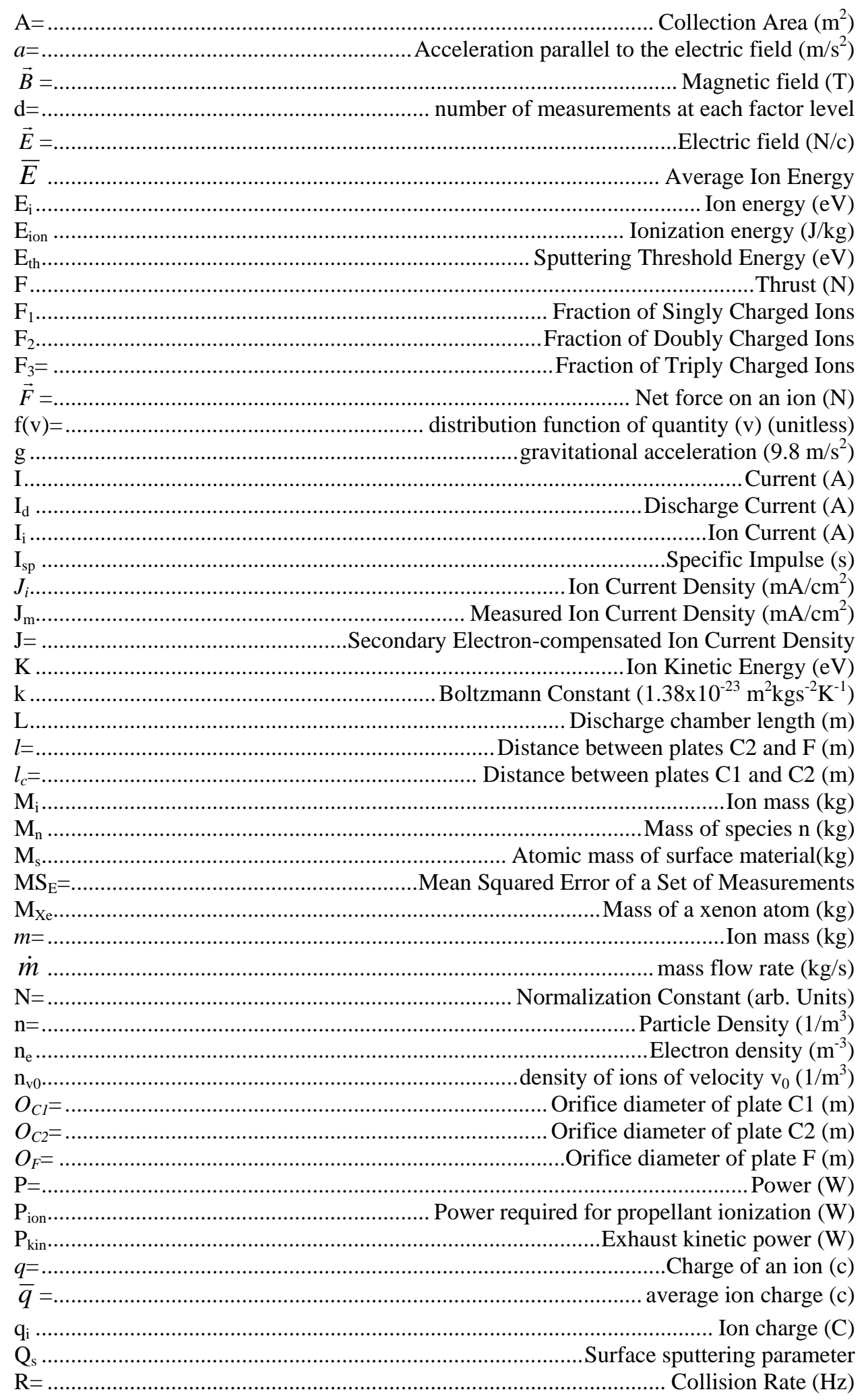




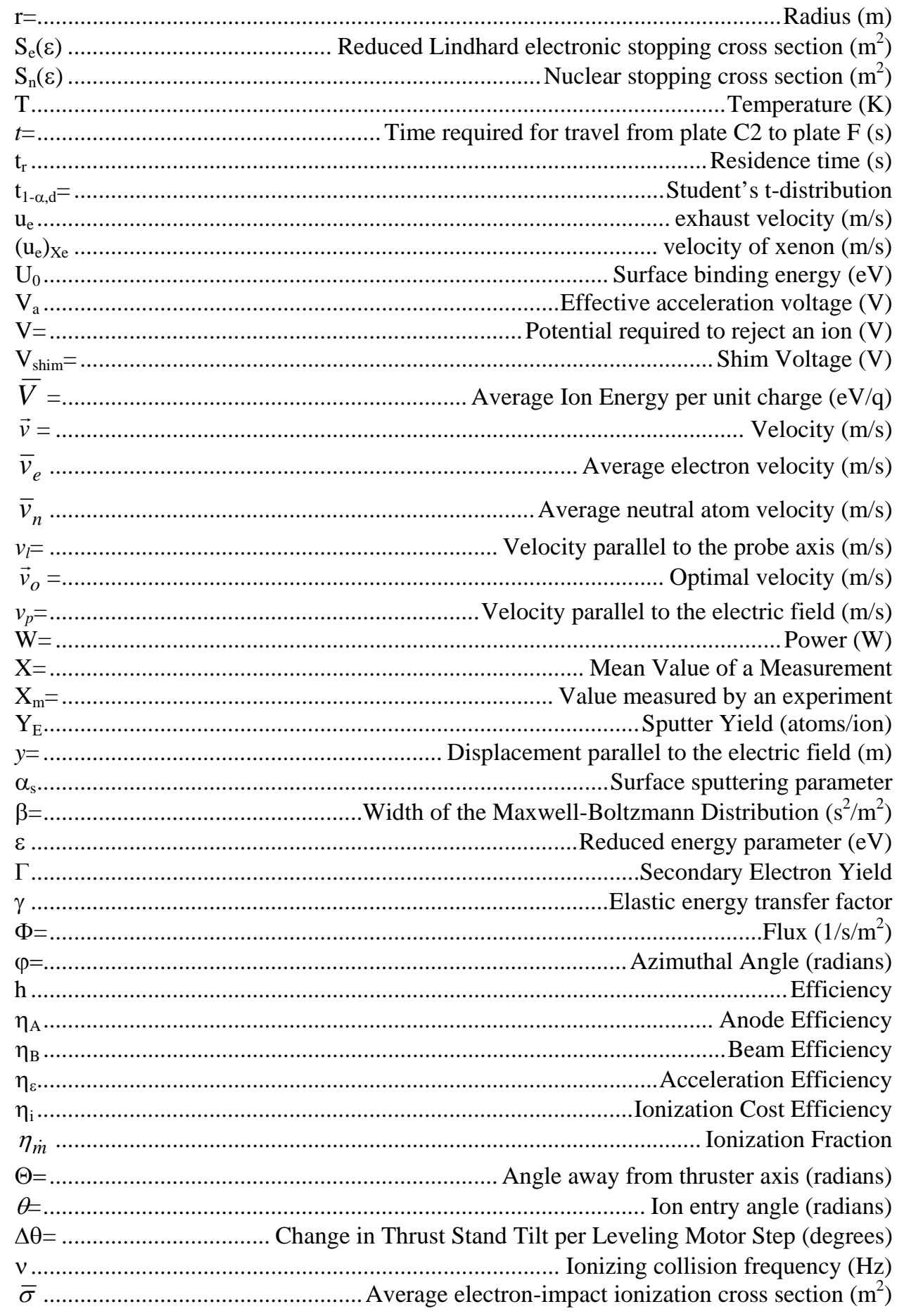




\section{Chapter 1: Introduction}

\subsection{Motivation for Electric Propulsion}

Electric Propulsion (EP) is a technology for in-space propulsion by which a propellant is accelerated by electrical means, rather than the purely thermal means utilized in chemical thrusters. ${ }^{1}$ Chemical rockets suffer from two fundamental limitations that EP is well suited to eliminate. There is a limited amount of power available in a given flow of chemical fuel. This energy limit is based on the enthalpies of formation of fuel, oxidizer (if any) and reaction products, and thus a given combination of fuel and oxidizer cannot provide more energy than the enthalpies allow. This limits the maximum temperature of combustion. In addition, the structure of a chemical rocket is limited in the amount of heat flux it can tolerate before failing. Increasing the temperature of combustion of the propellants directly increases the heat flux, thus materials considerations prevent chemical thrusters from operating at very high temperatures. Due to the fact that the acceleration mechanism in rockets is based on the temperature of combustion, this places a fundamental limit on how fast the reaction products can leave the rocket. Exhaust velocity has a direct effect on the amount of propellant required to accomplish a mission. This can be seen using the rocket equation:

$$
e^{\frac{\Delta v}{u_{e}}}=\frac{M_{o}}{M_{f}} .
$$

The $\Delta \mathrm{v}$ of a mission is directly related to the difficulty of the mission; the higher the $\Delta \mathrm{v}$, the higher the required total impulse from the propulsion system. Increasing the thruster exit velocity, then, will increase the ratio of initial to final mass, thus decreasing the amount of propellant necessary to apply a specified $\Delta \mathrm{v}$. The mass ratios required to perform a mission as a function of the mission $\Delta \mathrm{v}$ are plotted in Figure 1-1. $\Delta \mathrm{v}$ required to perform a low earth orbit to geosynchronous orbit (LEO-GEO) transfer and a mars ascent maneuver are included. 


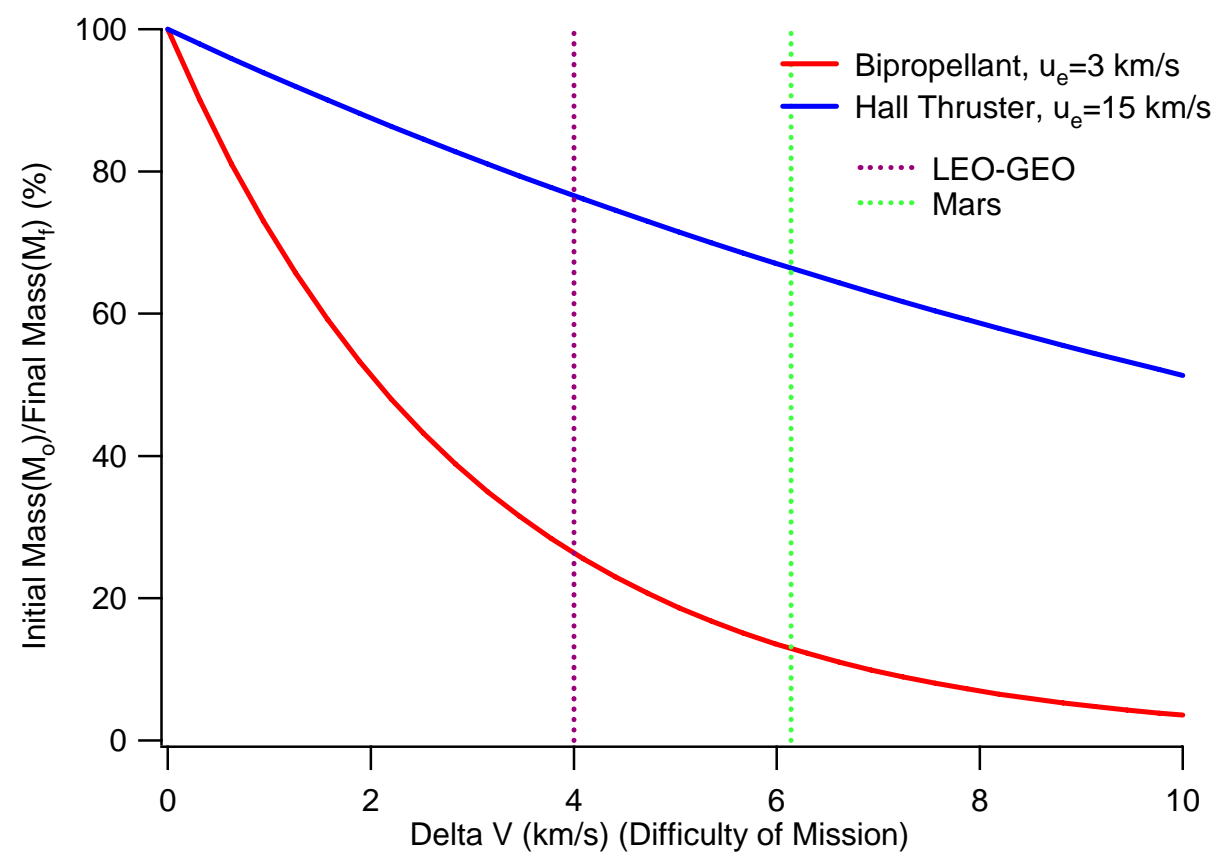

Figure 1-1: Mass Ratios as a function of mission $\Delta v$ for a chemical rocket and a Hall thruster

EP thrusters avoid the limitations of chemical rockets by decoupling the acceleration mechanism from the propellant. In EP thrusters, the propellant is accelerated through application of electrical power, either in the form of electro thermal heating or through ionization and acceleration by electric or magnetic forces. Use of electrical power for electrothermal heating of propellant suffers from the same materials constraints as chemical rockets, and the maximum exhaust velocity is limited. Electromagnetic means of acceleration, however, do not require physical constraint of high-temperature propellant flows, as in chemical rockets. Removing the dependence on material thermal properties allows very high acceleration of propellants. Simply increasing the electromagnetic field that accelerates the propellant ions may increase the exhaust velocity of the thruster. Thrusters that utilize electric or magnetic means have very high exhaust velocities; Hall thrusters typically exhibit exhaust velocities in the range of 15,000-20,000 $\mathrm{m} / \mathrm{s}$. Gridded ion thrusters and magnetoplasmadynamic thrusters have exhaust velocities in excess of $30,000 \mathrm{~m} / \mathrm{s}^{2,3}$

Chemical thrusters used for in-space propulsion typically have exhaust velocities in the 2,0003,000 m/s range. ${ }^{4}$ Large primary-propulsion rockets have achieved exhaust velocities of up to 4,300 m/s. Replacing a chemical rocket with an EP system can greatly decrease the amount of propellant required for a mission, allowing for either a smaller spacecraft or an increased payload mass fraction. Additionally, 
electric propulsion may be enabling for very long missions with a very high $\Delta \mathrm{v}$ requirement. This is because the propellant mass fraction for a system with a low exhaust velocity becomes prohibitive at very high $\Delta \mathrm{v}$, requiring that a propulsion system with a very high exhaust velocity be used.

A significant drawback to EP thrusters is that the thrust force is very low. This can be seen from the equation:

$$
T=\frac{2 \eta P}{u_{e}} .
$$

Thus for a given thruster power and energy efficiency, a high exhaust velocity will result in a lower thrust force than would be seen for a low exhaust velocity. Thus for a thruster using $2 \mathrm{~kW}$ of power and operating at $50 \%$ efficiency with an exhaust velocity of $15,000 \mathrm{~m} / \mathrm{s}$, the thrust will be $0.133 \mathrm{~N}$. The power available to the thruster cannot necessarily be increased. In chemical systems, thrust can be increased by increasing the rate at which fuel is consumed, thus increasing the power but reducing burn time. This means that maneuvers can be performed more quickly due to the large thrust forces involved, but does not remove the limitations of low exit velocity inherent to chemical systems. Furthermore, chemical rockets are energy limited. As the energy for production of thrust comes from the propellant itself, for a given propellant load a finite amount of energy is available. EP thrusters cannot be scaled up this simply. Spacecraft have limited amounts of power available, and thus a thruster cannot use the large amounts of power required for high thrust and high exhaust velocity. Thus EP is limited in how much power can be used at a time, unlike chemical systems where power is effectively unlimited. EP systems, however, have effectively unlimited energy. Due to the decoupling of propellant and the energy required for acceleration, EP can increase the energy imparted to the propellant without affecting total power. This does not eliminate the issue of limited power, however, and prevents EP from seeing use as a launch technology, as the thrust forces cannot overcome the pull of gravity at reasonable power levels. EP is extremely well suited to use in a space environment, however, as the thruster does not need to fight gravity directly and there is little or no atmospheric drag to overcome. 


\subsection{Hall Effect Thruster Overview}

A Hall effect thruster is a coaxial device that uses electrostatic forces to accelerate an ionized propellant, which typically uses xenon gas as propellant. ${ }^{5,6}$ A diagram of a typical thruster is in Figure 1-2. Thrusters of this type have been under development since the early 1960s. ${ }^{7}$ Thrust forces from a Hall thruster are typically in the tens to hundreds of millinewtons. ${ }^{8,9}$ Hall thrusters have since been used primarily for station keeping maneuvers on satellites, but have also been used for primary propulsion on scientific missions. ${ }^{10}$

The electric field in a Hall thruster is supplied by an anode, which is placed in the bottom of a ring-shaped ceramic discharge channel. Surrounding the discharge channel is a magnetic circuit, made up of electromagnet coils and ferromagnetic cores and poles which direct the magnetic field radially through the discharge channel. The magnetic field shape is such that the field strength is at maximum near or slightly upstream of the thruster exit plane. A charged particle entering this region is trapped by the fields and forced to move perpendicular to both; in this case circumferentially. Whether a particle will become trapped is dependant on the field strength and the particle mass. Particles trapped in a uniform magnetic field will move in a circular path with a radius defined as the Larmor radius. This radius can be calculated from the equation:

$$
r_{l}=\frac{m v}{q B}
$$

where the velocity used for the calculation is only the component of velocity perpendicular to the magnetic field. A condition for trapping a particle in a Hall thruster discharge, then, is that the Larmor radius of the particle is significantly smaller than the physical dimensions of the thruster. The magnetic field, then, is tuned such that the discharge electrons have a very small Larmor radius and are thus trapped, while the much more massive ions, with their correspondingly higher Larmor radii, will be able to escape relatively unimpeded. Electrons are provided to the thruster by an external cathode, which also functions as the beam neutralizer. The direction of the magnetic field is unimportant to thruster operation; reversing the field direction merely reverses the electron motion, and the effectiveness of the electron trap is unaffected. 
Propellant is supplied to the discharge channel through the anode. The neutral atoms experience no net force from the electric or magnetic fields, and diffuse from the anode towards the thruster exit. As the propellant atoms travel through the cloud of trapped electrons, the atoms are ionized by electron impact, producing positively charged ions and additional free electrons. The newly-formed propellant ions now feel a net force from both the electric and magnetic fields in the thruster. A xenon ion has roughly 240,000 times the mass of an electron, which means the magnetic field in the thruster is far too small to trap the ions, thus the ions are accelerated out of the thruster by the electric field. The electric field, then, presents a 'potential hill' for the propellant ions. Ions are created at the top of the hill, and effectively roll down this hill, becoming accelerated according to the height of the hill.

\subsection{Motivation for Alternative Propellants}

Xenon has many attractive qualities as a Hall thruster propellant. Some of these advantages are low ionization cost, high atomic mass, and ease of propellant supply. Ionization cost is of high importance to thruster operation, as any portion of thruster power required to ionize the propellant is

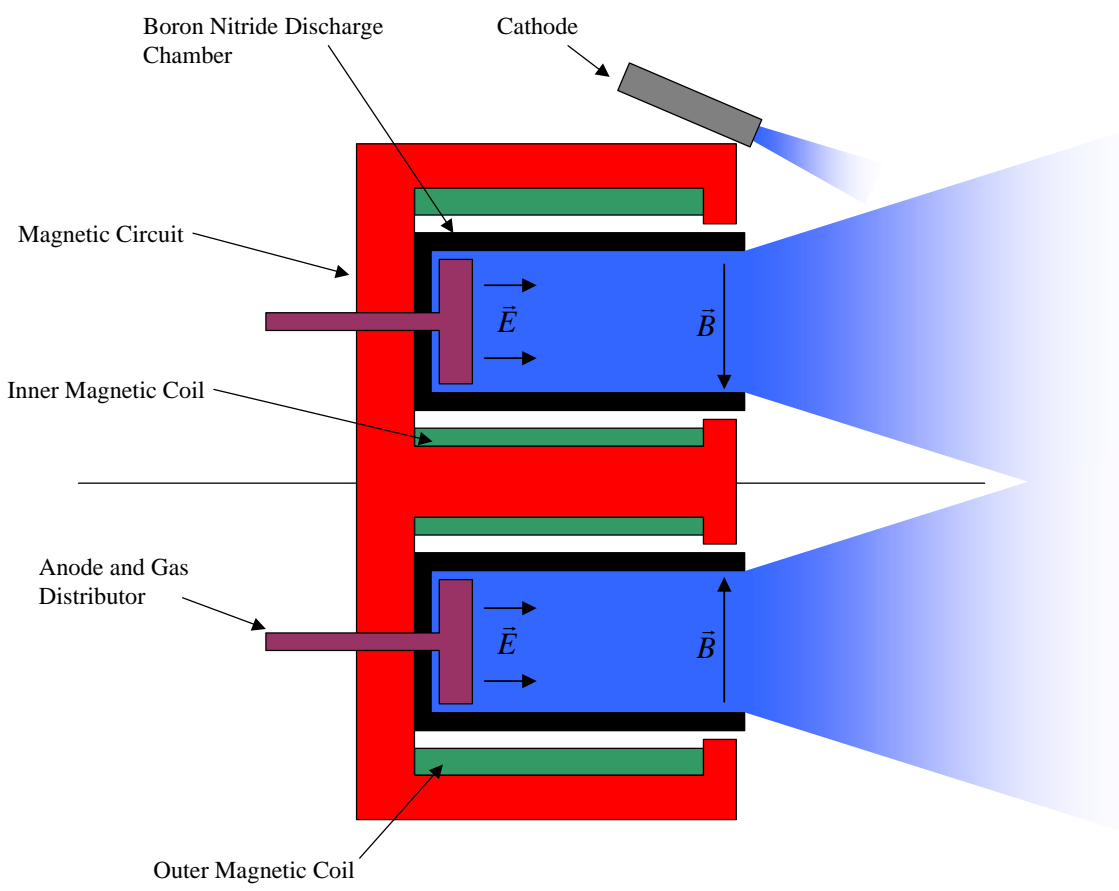

Figure 1-2: Cross Sectional View of a typical Hall thruster 
unavailable for propellant acceleration, and represents a direct efficiency loss. Thus xenon can achieve a much higher theoretical efficiency than other monatomic gases such as krypton or argon due to its low ionization energy. The use of molecular gases as a propellant can be problematic, as electron impact events may not ionize the propellant, rather break the chemical bond between atoms and be a further loss of efficiency, thus monatomic propellants are preferred. High propellant mass is advantageous in that heavy atoms will provide more thrust than small atoms, on a per-atom basis. This is based on the electrostatic acceleration in a thruster; under the assumption that ions gain the same energy rolling down the potential hill represented by the thruster's acceleration process, heavy atoms will move slower than light atoms. This relationship is based on the square root of the mass, however, while thrust is based on the product of mass and velocity. Thus the thrust is adjusted by the propellant mass divided by the square root of the propellant mass; a net increase for increasing mass. Xenon is the heaviest monatomic gas, thus it is the best gas for achieving high thrust. Xenon and other gases are very easy to supply to a thruster; propellant mass flow control is accomplished through commercially available mass flow controllers, and thus a precisely controlled flow of propellant can be maintained to the thruster with minimal effort. A complete analysis of this is presented in Appendix 1.

Xenon is not without drawbacks, however. The two most important issues with xenon use are propellant cost and testing facility cost. Xenon is a very expensive gas, thus testing of large-scale thrusters, extended lifetime tests, or long missions have significant propellant costs. These costs can become prohibitive for very long missions. The benefit of a less expensive propellant, then, is a direct cost savings in testing and flight of a thruster.

The gaseous nature of xenon, which makes it so favorable from a feed system standpoint, causes serious issues with ground testing. Thruster exhaust must be removed from the facility in order to maintain the facility at a space-like vacuum.. With gaseous propellants, this involves the use of large and costly pumping hardware. As the required pump throughput increases linearly with propellant mass flow (and hence, thruster power), testing of very large thrusters requires very large pumping systems. By using a condensible propellant, this problem can be avoided. Condensible propellants are those which are a solid or liquid at room temperature, and must be heated and evaporated before injection into a thruster discharge. Unlike gaseous propellants, a condensible propellant is in effect 'self pumping,' and does not require active 
removal from the vacuum facility. Rather, the propellant exhausted from the thruster will freeze upon impact with the walls of the facility. The only active pumping required, then, is only that which is necessary to achieve and maintain vacuum, and is independent of thruster operation.

\subsection{The Direct Evaporation Method}

Through the analysis of the energetics of several candidate propellant options, as presented in Appendix 1, bismuth has emerged as a propellant that is well suited to Hall thruster operation. Bismuth is a favorable propellant due to several factors, including ionization energy and testing costs. The ionization energy of bismuth is $7.2 \mathrm{eV}$ per atom, which is significantly less than the $12.1 \mathrm{eV}$ ionization energy of xenon. This will manifest as a direct energy savings to the thruster, and allow for higher theoretical efficiencies as less of the thruster's energy supply is required to ionize the propellant. The cost savings are manifested as savings in both facilities and the propellant itself. Since bismuth is a condensible propellant, the thruster exhaust does not need to be actively pumped from the test chamber, rather it will condense on the chamber walls. Due to the great expense of pumping in construction and operation of the vacuum facilities required for EP testing, elimination of the need to actively pump exhausted propellant will be a major cost savings. Additionally, the cost of bismuth is much less than that of xenon, allowing extended thruster testing at a much lower initial cost in propellant. Although immature as a Hall thruster propellant, the use of bismuth is not without precedent. ${ }^{11}$ Soviet work performed in the 1970's and 1980's evaluated bismuth anode layer thrusters. TsNIIMASH researchers reported thrusters with a power level up to $140 \mathrm{~kW}$ and exhaust velocities on the order of $80,000 \mathrm{~m} / \mathrm{s}$ at anode efficiencies exceeding $70 \%{ }^{12}$ The anode layer thrusters in these studies differ from the SPT-type thruster studied here in that the discharge channel of an

anode layer thruster is shallow and the walls are also the anode surface. SPT-type thrusters utilize an anode at base of a deeper channel than anode-layer thrusters, with the channel walls made up of ceramic. More recently, efforts under the Very High Impulse Thruster with an Anode Layer (VHITAL) program have made progress towards development of a very high specific impulse, high power thruster. ${ }^{12}$

Bismuth presents its own engineering difficulties, however. In order for the thruster to operate, there must be a precisely controlled source of bismuth vapor. Previous work used a heated bismuth reservoir that fed vapor directly into the thruster. The difficulty with this method was that the reservoir 
required significant resistive heating to maintain a temperature high enough to vaporize bismuth in sufficient quantities (more than 1,000 K), as the vapor pressure of bismuth is the driving factor in the evaporation rate and is dependent on temperature. These resistive heaters present a direct power loss to the thruster, as the power required for the heaters is nonpropulsive, and thus does not contribute to the performance of the thruster. In order to reduce or eliminate the need for resistive heating, a technique has been developed that utilizes the waste heat from the thruster to warm the bismuth reservoir to the required temperatures. ${ }^{13,14}$ In this design, the main anode of the thruster functions as the bismuth reservoir. This design has the potential to eliminate the need for resistive heaters during operation due to the fact that the plasma electrons heat the anode. This will allow the anode to be maintained at a temperature sufficient for bismuth evaporation purely from the discharge waste heat. The evaporation rate is controlled through the reservoir temperature and the permeated area through which the bismuth vapors diffuse into the discharge chamber. Since it is not practical to vary the vapor escape area through the reservoir mechanically, the mass flow rate, $\dot{m}$, is controlled by varying the reservoir temperature within the thruster. The evaporation rate is governed by the equilibrium vapor pressure of the liquid metal with the resultant goal of maintaining the appropriate evaporator temperature, which when combined with the open area produces sufficient quantities of bismuth vapor. Previous studies have found that for an anode with a $10 \%$ open-area fraction in a thruster of the type investigated in this paper, a temperature of $750{ }^{\circ} \mathrm{C}$ is sufficient to provide the necessary bismuth mass flow. ${ }^{14}$

Control of anode/reservoir temperature is necessary to control the flow of bismuth. If a traditional Hall thruster anode were replaced with a bismuth-carrying anode, any increase in temperature over the desired operation point would increase the bismuth flow. This increased flow would result in a higher discharge current, which in turn would increase electron flux to the anode, further increasing the anode temperature. This would in turn cause an even higher bismuth evaporation rate, which would continue in a positive feedback loop of increasing temperature, current, and heat into the anode. To maintain the anode at the desired temperature, an active control mechanism must be in place to either increase the heat loss from the anode or decrease the heat flux from the plasma electrons. Changing the heat loss from the anode is problematic, so adjustment of the incoming electron heat flux will be used to control the anode temperature. In order to control the temperature of the main anode, a pair of inert shim electrodes can be 
added to the discharge channel of the thruster, downstream of the main anode, as shown in Figure 1-3. By slightly varying the shim electrode voltage with respect to the main anode/reservoir, the discharge current can be shifted between the shim electrodes and the main anode. This shift in discharge current will then shift the discharge heating, with the result that shifting current to the shims will cool the main anode, and diverting current back to the anode will increase its temperature, and hence the bismuth evaporation rate. ${ }^{15}$ This thermal control strategy has been successfully implemented in a Hall thruster, and the temperatures achieved by the anode and the shims are plotted in Figure 1-4 for a typical experiment with the thruster. ${ }^{16}$

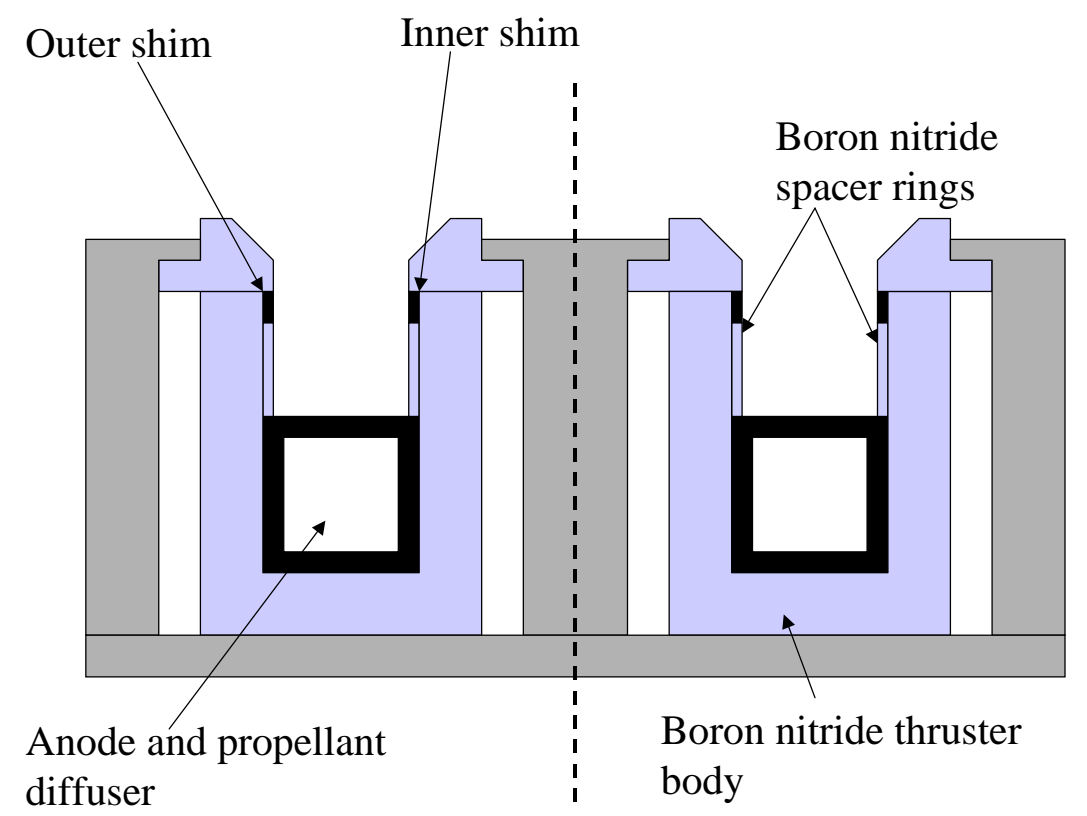

Figure 1-3: Cross-section view of the thruster with shim electrodes and main anode. 


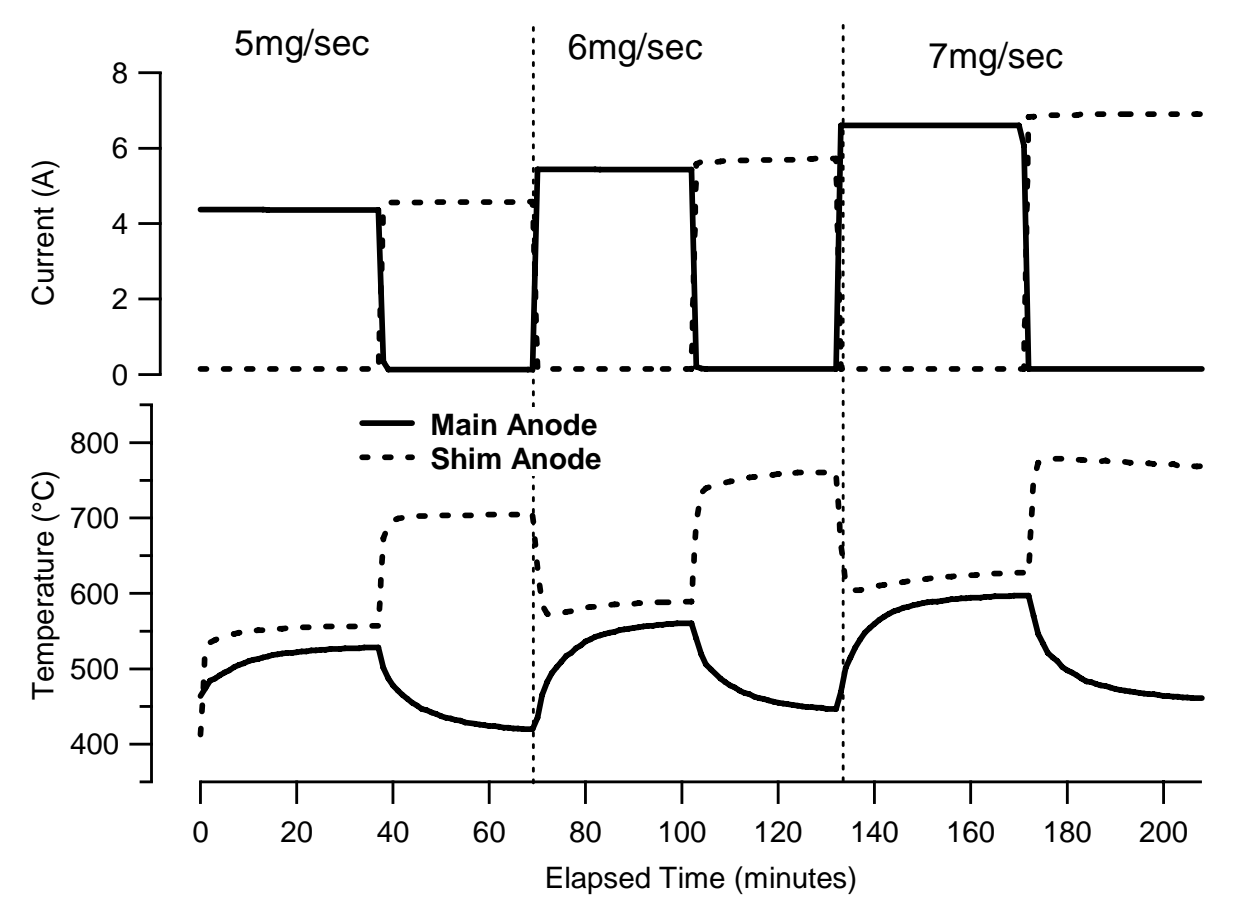

Figure 1-4: Temperature and current deposition on both the main and shim anodes as a function of
time for a thruster operating at $400 \mathrm{~V}$

The shim electrode configuration proposed here is part of a larger thruster design strategy

involving what are known as segmented anodes, where the thruster does not have a single main anode, rather two or more anodes which can be independently controlled for various purposes. Segmented anodes have been implemented in Hall thruster development for several purposes not related to thermal control. One use of segmented electrodes in previous studies was as a method of achieving two-stage operation, ${ }^{8,9}$ where the shim electrodes are maintained at a potential between the anode and the cathode, allowing for an acceleration-only stage which would allow the propellant to be accelerated to very high velocities. A diagram of one two-stage thruster design is in Figure 1-5. Other experimental investigations have been performed on segmented-electrode Hall thrusters which attempted to improve the performance of the thruster. ${ }^{17,18,19}$ These investigations showed that the divergence of the thruster plume could be improved through the use of segmented electrodes, as shown in Figure 1-6. The thruster used for the segmented electrode experiments is displayed in Figure 1-7. The investigations in these previous studies are very different in implementation of the segmented electrodes than the thermal control strategy proposed here. In all of the previous research on segmented electrodes, the goal was a performance increase; either through an increase in exhaust velocity from two-stage operation or improvement of the beam divergence and other 
characteristics. The effect of segmented anodes when used for thermal control of the main anode on the performance of the thruster is not fully known, and represents a significant area of interest for future bismuth thruster development. Experiments performed on a similar thruster design operated with segmented anodes as a thermal control mechanism showed little change in thruster performance or beam profile. ${ }^{16,20}$ Additionally, the experiments showed that while the thruster design used could not achieve the absolute temperatures expected to be necessary for bismuth evaporation, a control authority of nearly $100 \mathrm{C}$ was available on the main anode, allowing for significant throttling capability.

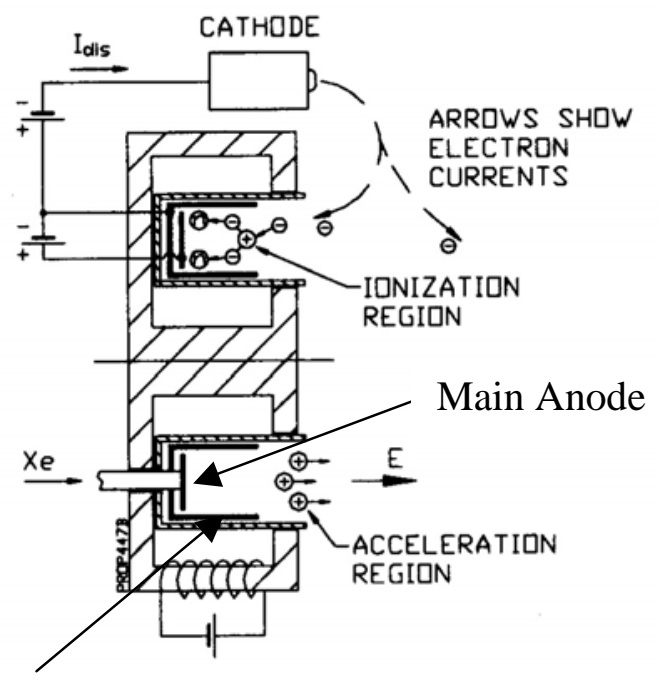

Shim Anodes

Figure 1-5: Diagram of a two-stage Hall thruster as used in Ref. 8 


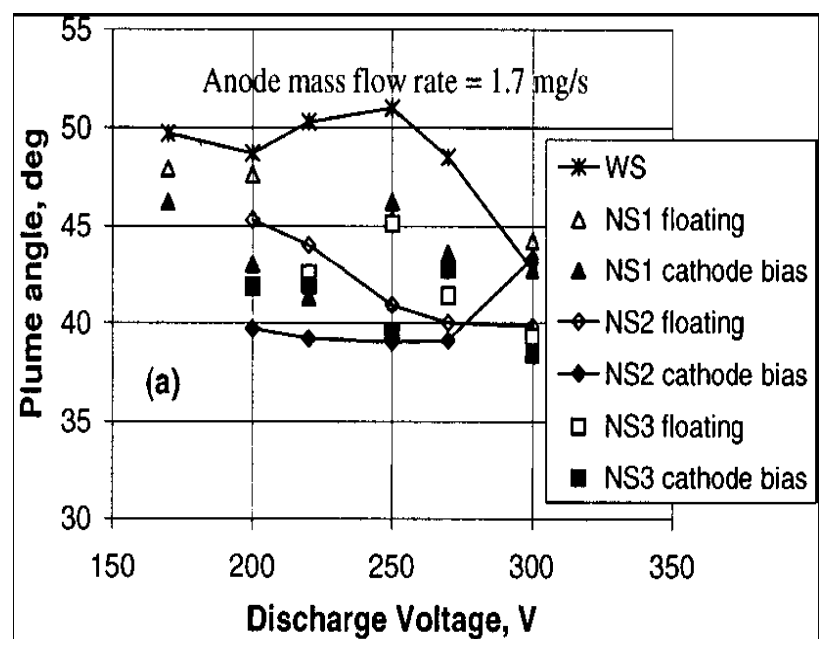

Figure 1-6: Observed changes in plume divergence for several segmented electrode configurations, from Ref. 18

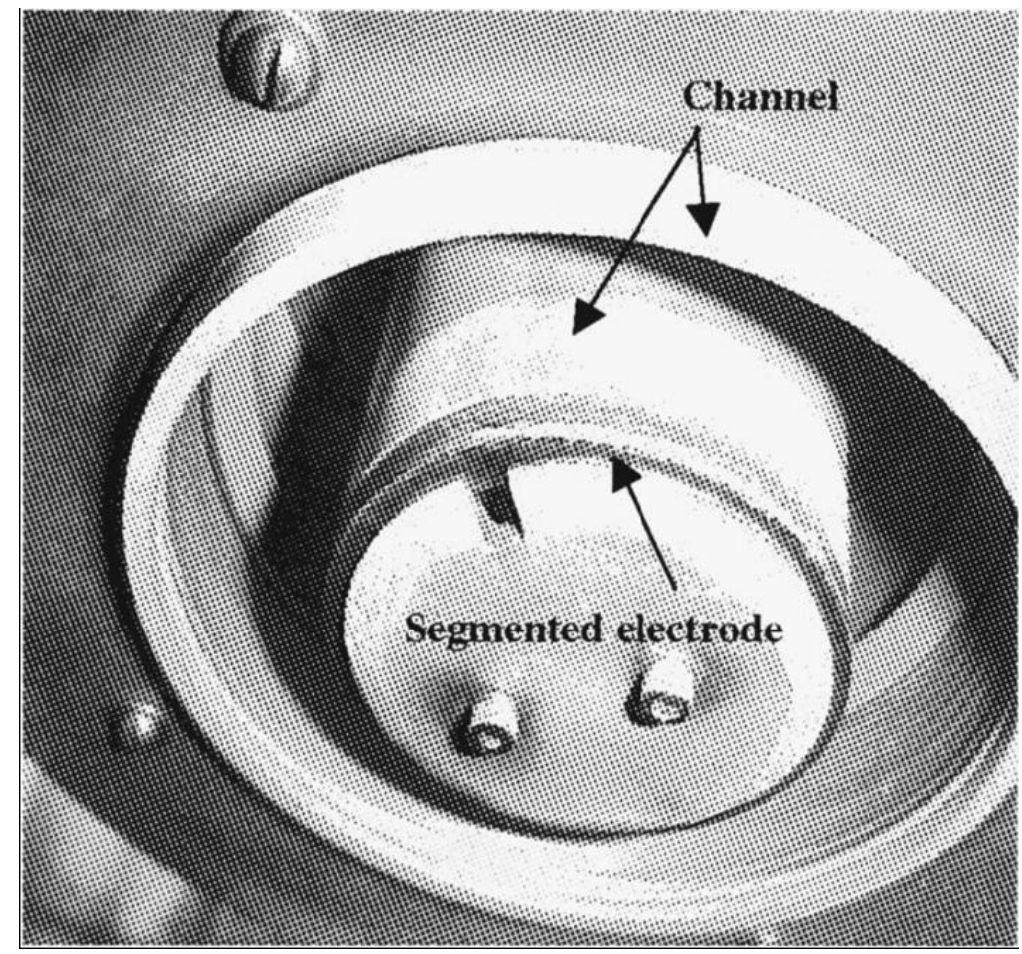

Figure 1-7: Photograph of the segmented anode thruster used in Ref. 18

\subsection{Contribution of Research}

The research reported here will contribute to the body of knowledge about Hall thrusters by determining the performance of a segmented electrode thruster as the discharge current is shifted between the main anode and the shims. This is unique in that previous efforts at utilizing segmented electrodes were oriented toward optimizing the plume characteristics of the thruster ${ }^{17-19}$ or increasing the total acceleration 
voltage through a two-stage acceleration method. ${ }^{8,9}$ The use of segmented anodes for thermal control thus represents a new thruster design and operation strategy, and must be thoroughly characterized from a performance and plume standpoint. The future development of a bismuth thruster using segmented anodes for thermal control requires thorough knowledge of the behavior of the thruster itself, using known and well-characterized propellants, so the performance change from switching to bismuth can be determined. The analysis of the behavior of a segmented electrode thruster will be performed using two methods. The first of these is to measure the thrust produced by the thruster and determine the effective exhaust velocity and energy efficiency of the thruster. Doing so will evaluate the 'black box' performance of the thruster, and hence whether the benefit to a spacecraft is changed by the addition of the segmented anode thermal control strategy. The second way to determine if the thruster's operating parameters have changed is to use plasma probes to measure the behavior of the ions in the plume of the thruster. Changes in the plume divergence (the direction of ion velocity), ion energy (ion velocity), and the number of multiply charged ions can all have an effect on the 'black box' performance of the thruster, and as such can provide insight into the reason for any performance change with the proportional control of the current acceptance by the segmented electrodes. Thus the goal of the research reported here is to:

- Determine if the performance of a 2-kW Hall-effect thruster is affected by adjustment of the location of discharge current attachment and, hence, discharge electron heating.

- Characterize the plasma in the thruster exhaust using several plasma probes, to attempt to determine the source, if any, of the performance change 


\section{Chapter 2: Segmented Thruster Design}

\subsection{BPT-2000}

The BPT-2000 is a 2-kW Hall thruster which was developed by Busek and Primex (now

Aerojet), ${ }^{21}$ as shown in Figure 2-1. The thruster utilizes four electromagnetic coils at the periphery of the thruster. There is no center magnetic coil. Rather, a large ( 25mm-diameter) soft iron pole is placed at the axis of the thruster, allowing the return of magnetic flux to the back plate without the need for a separate magnetic coil.

The body of the thruster is constructed from boron nitride ceramic, with a ring-shaped discharge channel machined on one side. The thruster anode has a U-shaped cross section, and fills the entire discharge channel, as shown in Figure 2-2. Welded to the bottom-inside of the anode is the propellant distributor, which has a conical cross section and extends approximately halfway up the anode.

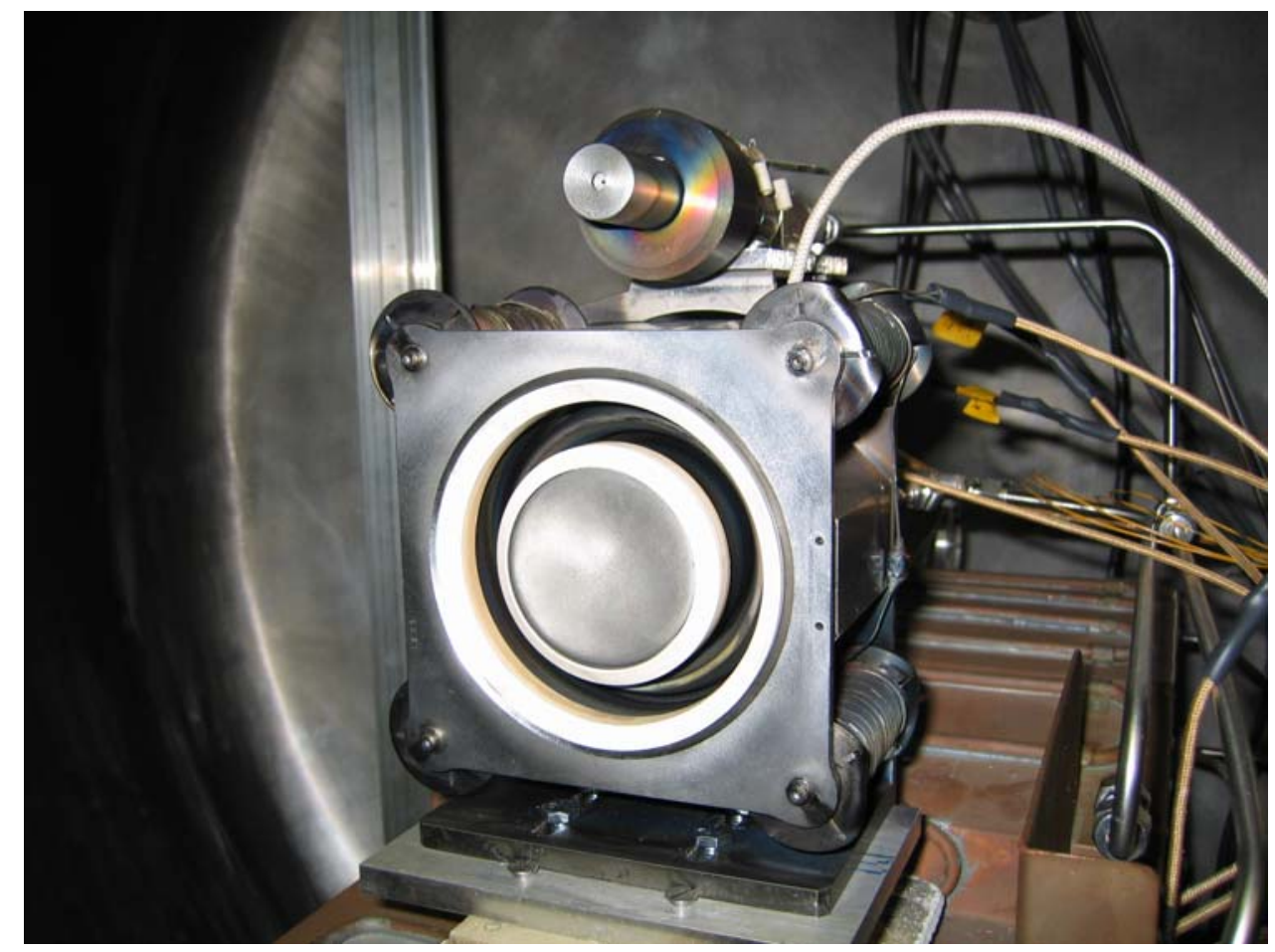

Figure 2-1: Photograph of the BPT-2000 with cathode 
The performance of the BPT-2000 has been characterized in Ref. 21 to be in the roughly $1750 \mathrm{~s}$ specific impulse range (varying between approximately 1450 and 1800 s) and approximately 45-50\% total energy efficiency over power ranges of 1,500-2,500 W and anode voltages of 300, 350, and $400 \mathrm{~V}$. The beam of the BPT-2000 thruster has been characterized to have a 52-55 degree half-angle required to enclose $90 \%$ of the beam current.

\subsection{Laboratory Thruster}

The thruster constructed for the experiments reported here is identical to the BPT-2000 in magnetic circuit and exterior dimensions. A photograph of the thruster is in Figure 2-3. The boron nitride body was manufactured to the same interior and exterior dimensions as the BPT-2000 body, such that the anode from a BPT-2000 could be inserted into the thruster without modification. The original anode was replaced with a much smaller main anode, which fills the center of the bottom half of the discharge channel, as shown in Figure 2-4. The new anode does not fill the entire width of the channel, rather it is significantly narrower to allow for a higher power density and thus higher anode temperatures. Shim electrodes were added at the end of the discharge channel, pressed against

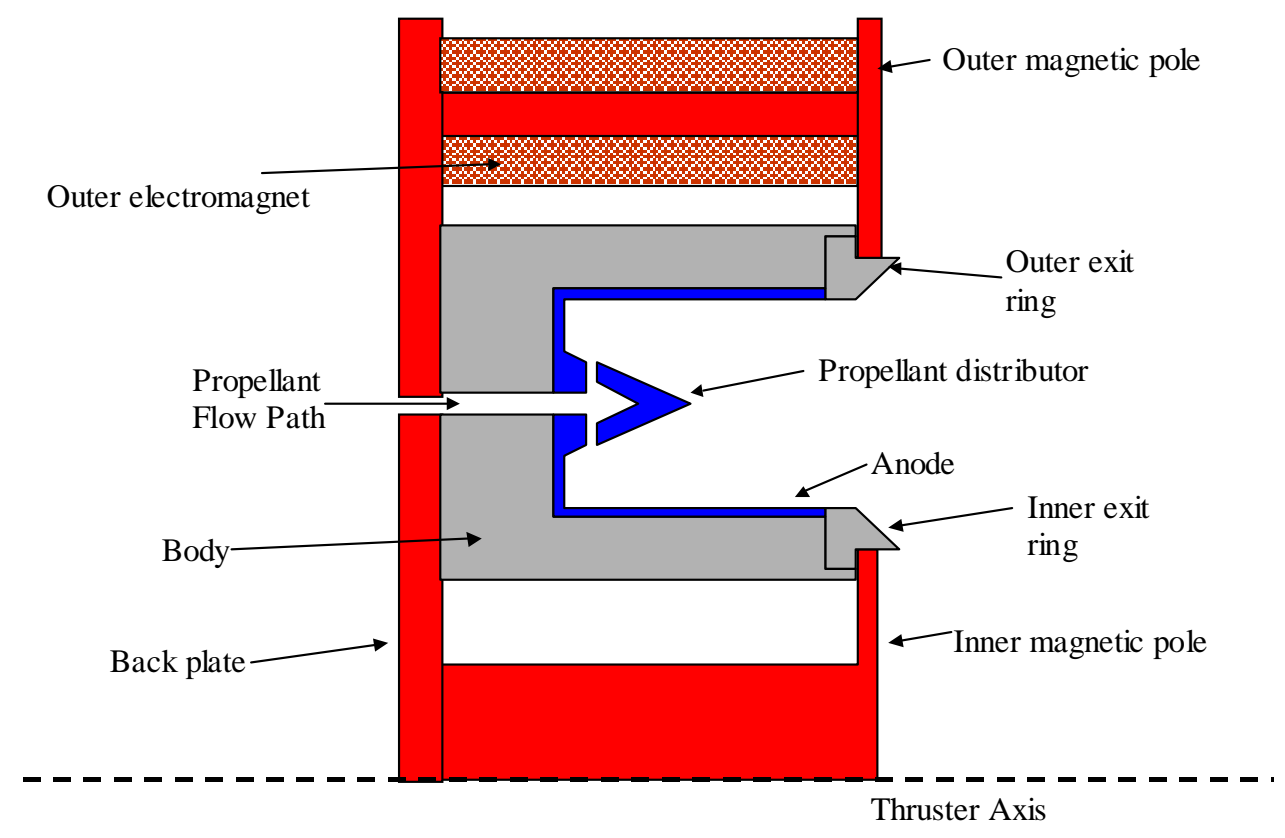

Figure 2-2: Cross section of the BPT-2000 Hall thruster 
the exit rings. Boron nitride spacers were used to fill the remaining space and hold the shims in a constant location away from the anode. The anode was fastened to the boron nitride body similarly to the original anode from the BPT-2000, and functioned as the propellant distributor.

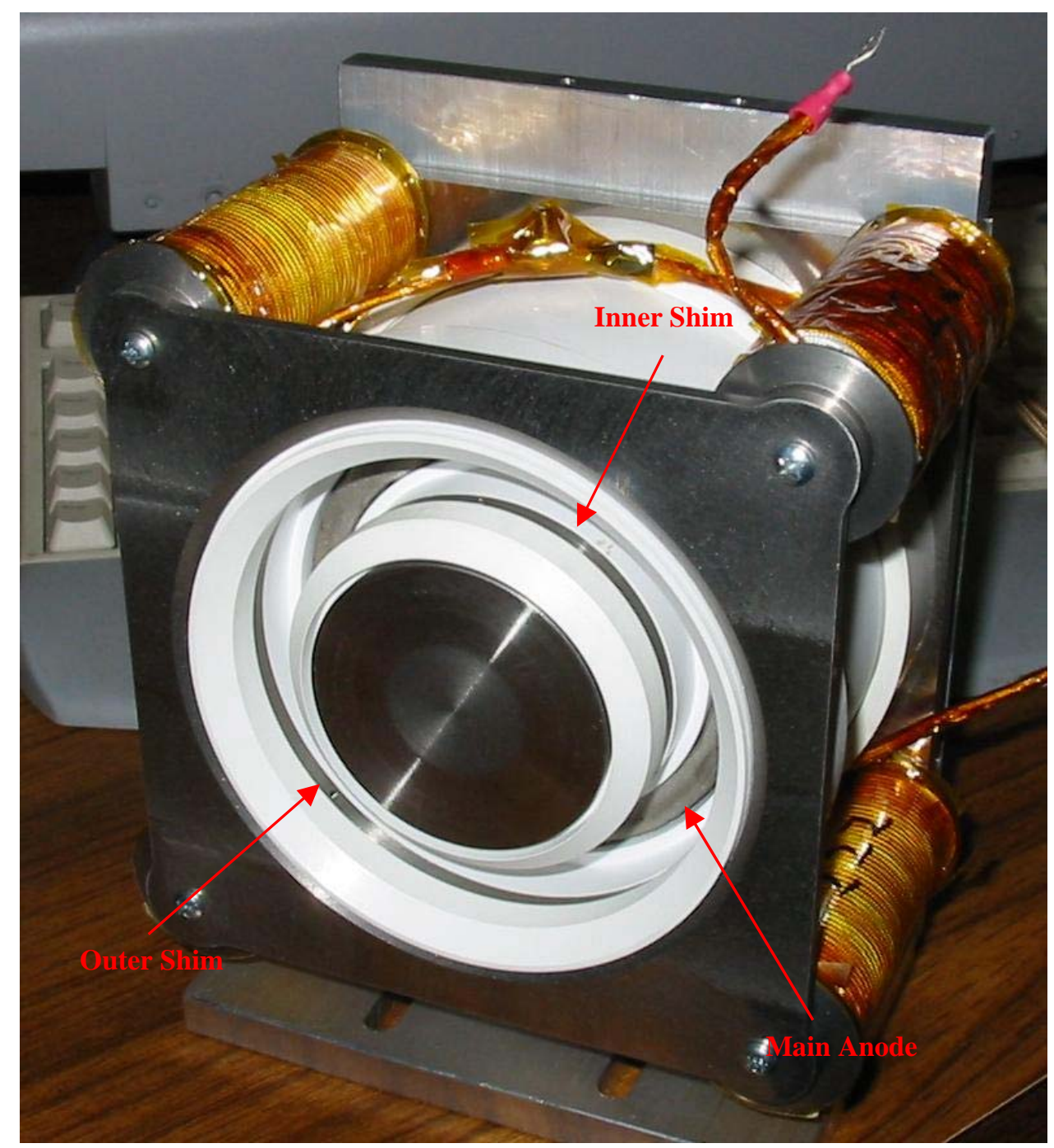

Figure 2-3: Photograph of the segmented electrode hall thruster 


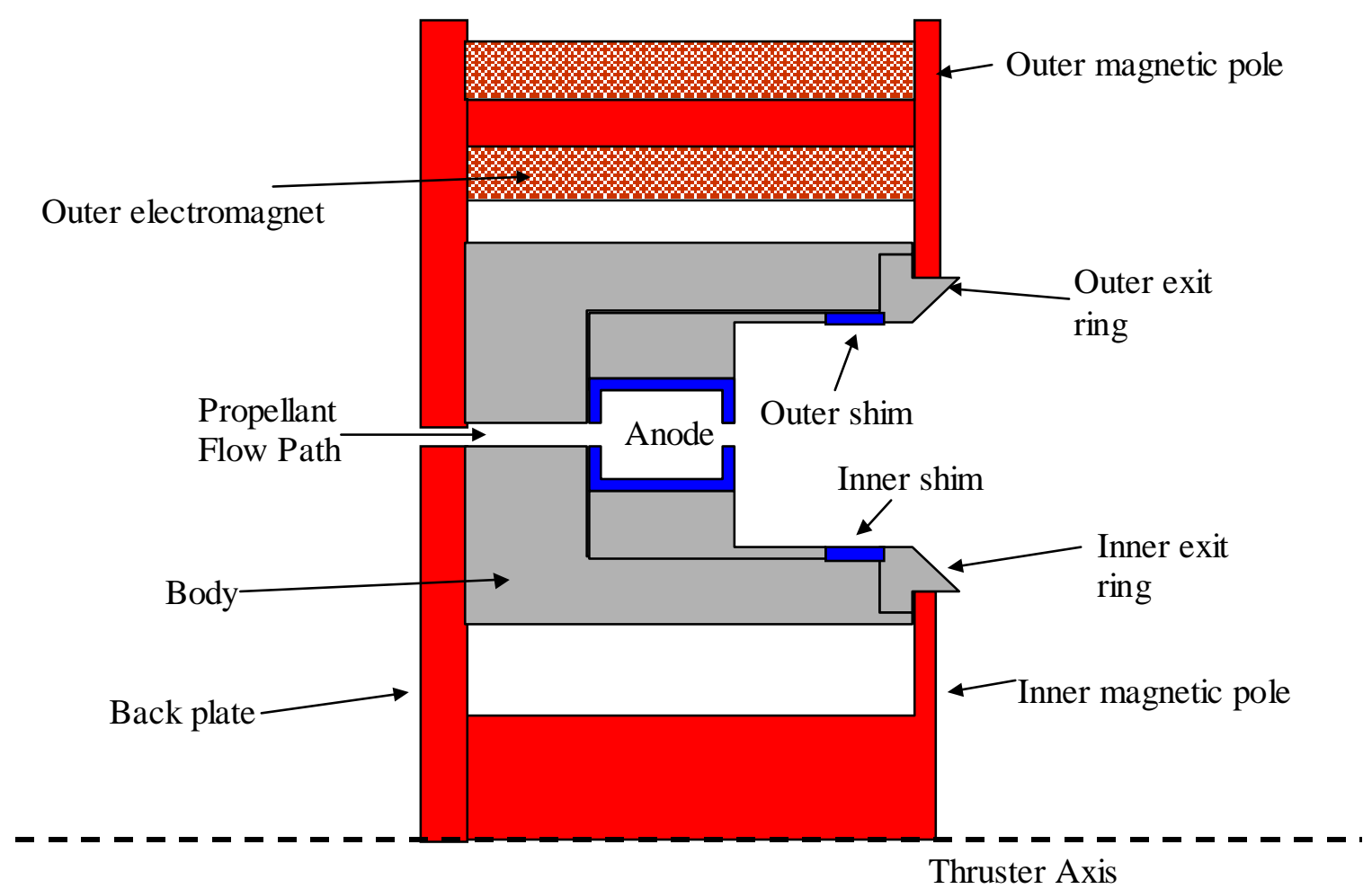

Figure 2-4: Cross section of the segmented anode thruster

\subsection{Cathode}

The laboratory cathode utilized for thruster operation consisted of a lanthanum hexaboride emitter pellet, enclosed in a titanium shell. A loop of tungsten outside the titanium shell functioned as a keeper electrode. The pellet was heated through tungsten resistive heaters. Thermal insulation was accomplished through use of molybdenum multilayer insulation, which served to greatly reduce radiative heat losses. Cathode operation consisted of heating the pellet to temperatures that would emit electrons while in the presence of a xenon flow. Once the cathode pellet became hot enough to emit electrons, a discharge was initiated between the cathode and the keeper. Once the cathode discharge was established, the thruster discharge could be initiated. The cathode was operated with xenon flow equal to $10 \%$ of the thruster mass flow. The heater and keeper were operated continuously throughout testing at low power levels, to maintain the cathode discharge in case the thruster discharge unexpectedly terminated. 


\section{Chapter 3: Performance Characterization}

There are three aspects of performance, which, while related, can each provide unique insight into thruster operation. These aspects are thrust, specific impulse, and efficiency. Thrust is of obvious importance to thruster operation. Specific impulse is defined as the total impulse provided by a propulsion system per unit weight of propellant. Total impulse provided by a thruster system is defined by:

$$
I=\int F d t
$$

Eqn. 3-1

In an EP system, however, the assumption can be made that thrust is constant for a given operating point, allowing for removal of the integral notation. Specific impulse is the total impulse provided per unit weight of propellant, and can be found by:

$$
I_{s p}=\frac{F t}{m g} .
$$

This can be further simplified by substituting for mass flow, giving the solution:

$$
I_{s p}=\frac{F}{\dot{m} g} \text {. }
$$

This solution is very similar to the equation for velocity as a function of the mass flow and thrust force of a fluid flow, which is calculated by:

$$
u_{e}=\frac{F}{\dot{m}}
$$

The only difference between the equations for specific impulse and flow velocity is that the gravitational constant $g$ has been added. Thus the specific impulse is a measurement of the average exhaust velocity of the thruster. A change in specific impulse thus can determine if the thruster acceleration mechanism is changing. It is expected, then, that specific impulse will change with shim and main anode voltages, as these quantities both directly affect the electric field in the thruster and thus the potential hill present. It is not expected that specific impulse will be dependant on thruster mass flow, so the specific impulse measurement is effectively removing mass flow from consideration of thruster performance. Moreover, as the exhaust velocity (and thus, specific impulse) has a direct effect on the amount of propellant a spacecraft 
must carry for a given mission as in Eqn. 1-1, the characterization of specific impulse will help determine if the proportional control of segmented anodes will affect the propellant requirements for a space mission.

The third aspect of thruster performance that will be considered is efficiency. This is calculated through Eqn. 1-2, which can be solved for efficiency, yielding the equation:

$$
\eta=\frac{F u_{e}}{2 P}
$$

Exhaust velocity has already been calculated in terms of thrust force and mass flow in Eqn. 3-4, however, and can be substituted to yield:

$$
\eta=\frac{F^{2}}{2 \dot{m} P}
$$

Thus the efficiency of the thruster can be calculated from thrust, mass flow and power. In an EP thruster power is equal to the output of the thruster power supplies. Several different values of thruster power can be used here, however. The thruster cathode and magnets typically require some power to operate, so total power used to calculate efficiency can be the sum of discharge, magnet, and cathode powers. This can complicate the analysis of the acceleration efficiency of the thruster, however, as magnet and cathode powers are non-propulsive and dependent largely on the specifics of their design. Thus only the thruster discharge power will be used to calculate efficiency. Discharge power is the product of voltage and current at the anode (and for a segmented thruster, the sum of power deposited on the main anode and the shims), so the efficiency when calculated with only the discharge power is called the anode efficiency.

\subsection{Vacuum Facility}

All tests were performed in the Xenon Test Facility (XTF) at Michigan Technological University. The facility is comprised of a 2-m-diameter by 4-m-long vacuum tank. Rough pumping is accomplished by a two-stage rotary oil-sealed vacuum pump with a Roots blower, capable of pumping at 200 l/s. High vacuum is achieved through dual 48-inch-diameter cryopumps, capable of a combined pumping rate of 120,000 l/s on nitrogen as in Figure 3-1. Tank pressure was measured via a Bayard-Alpert ionization gauge mounted behind the thruster, on one of the end-caps of the tank. Base pressure of the tank is below $7 \times 10^{-6}$ Torr $\left(5.25 \times 10^{-8} \mathrm{~Pa}\right)$, and the maximum pressure seen during testing was $3.84 \times 10^{-5}$ Torr $\left(2.88 \times 10^{-7} \mathrm{~Pa}\right)$, 
corrected for xenon. The probes utilized in the characterization of the thruster plume were mounted on a motion table with two linear axes, one parallel to the tank axis and one horizontal and perpendicular to tank axis. Rotation about the vertical axis was accomplished through use of a rotary stage, as shown in Figure 3-2. The motion tables were controlled via servomotor.

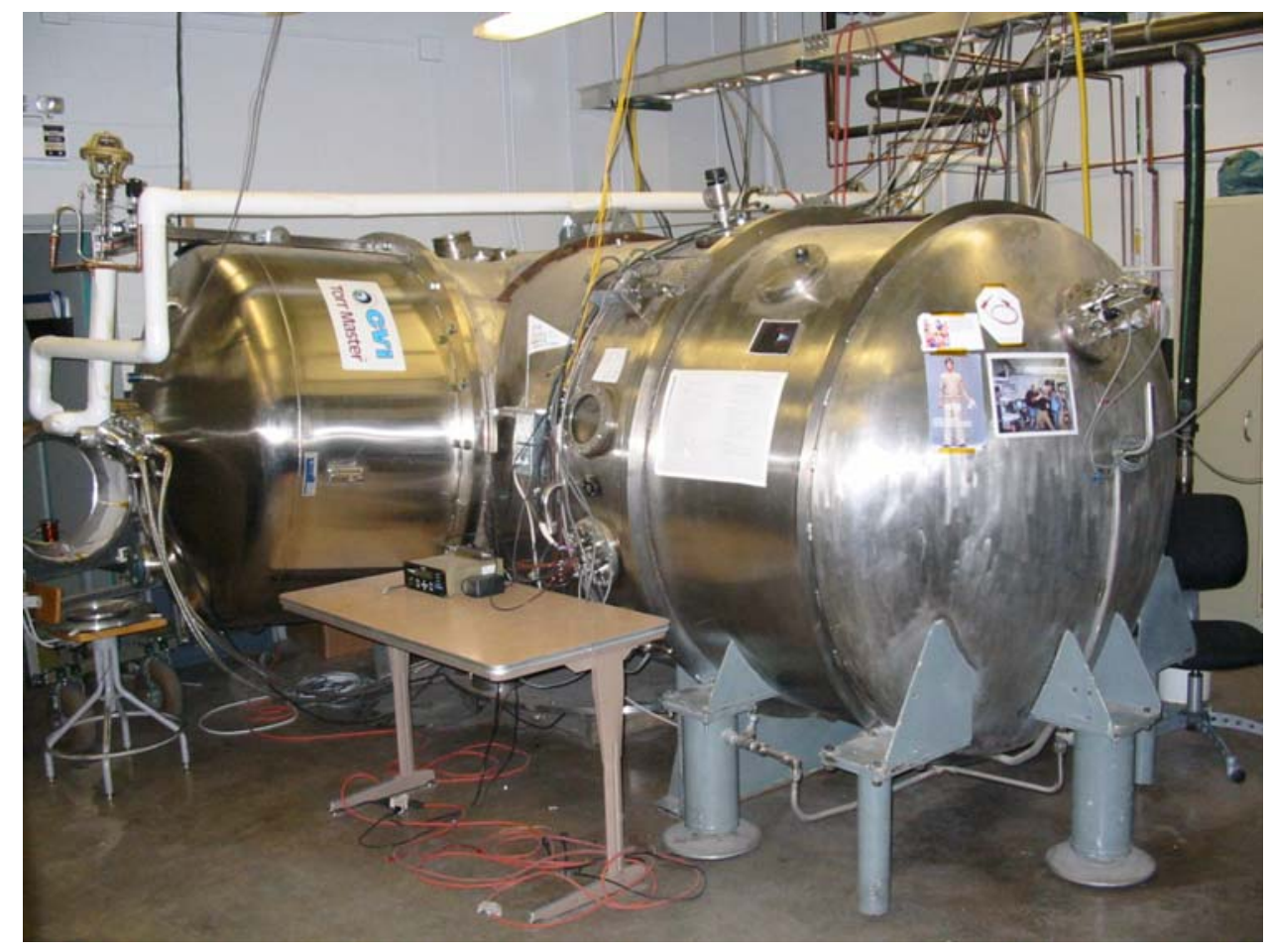

Figure 3-1: Photograph of the XTF 


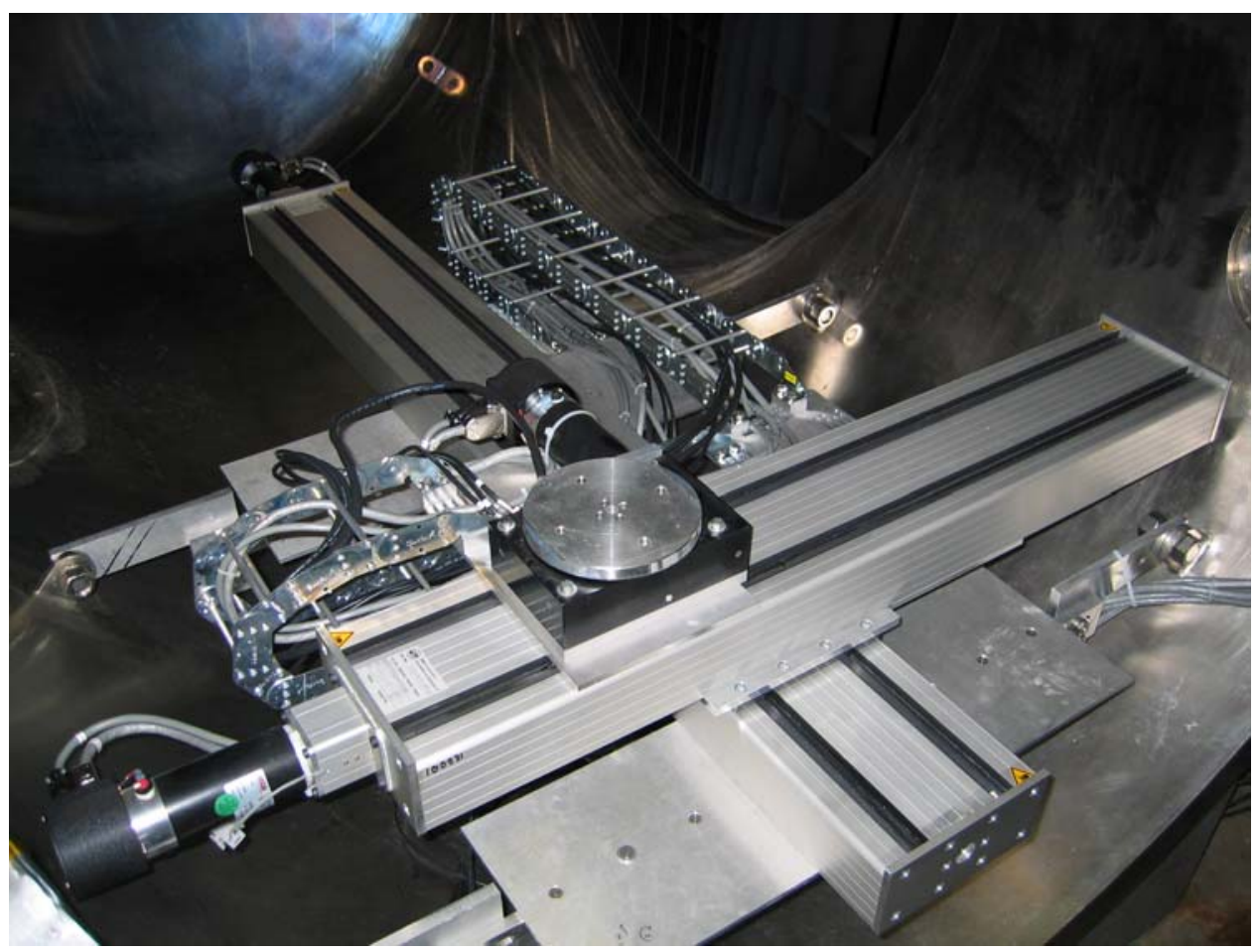

Figure 3-2: Photograph of the motion control table inside the XTF

\subsection{Thrust Stand}

A NASA-Glenn-style ${ }^{22}$ inverted pendulum thrust stand was utilized for thrust characterization, as shown in Figure 3-3 and Figure 3-4. The thrust stand takes advantage of the nature of a pendulum, that there are two equilibrium points where the pendulum will remain if unperturbed. The most well-known of these points is pointed downward. This state is the most stable position, as any perturbation which moves the pendulum from this state will cause the pendulum to swing through this point until friction, air resistance, and other drag forces reduce its energy to the point where it is stationary at the downwardpointed position again. The second equilibrium point is pointed directly upward. This is inherently unstable, as any perturbation will cause the pendulum to swing downward, away from its initial position. The pendulum will then swing normally until it is slowed by drag and goes to the downward-pointed equilibrium state. The inverted pendulum thrust stand places the thruster on top of a pendulum in the upward-pointed, unstable equilibrium state. This allows the small thrust forces produced by the thruster to cause large changes in displacement, which are then read by a linear variable displacement transducer 
(LVDT). The thrust stand is maintained in the upright position by a retention spring. The thruster mounting plate is supported by leaf springs, which allow the thruster mounting plate to move while assisting the retention spring in maintaining the thrust stand in the upright position. The system is extremely sensitive to vibration due to its unstable equilibrium and retention via spring force, so an electromagnetic damper is used to control motion of the thrust stand, effectively eliminating vibration from the thrust measurements.

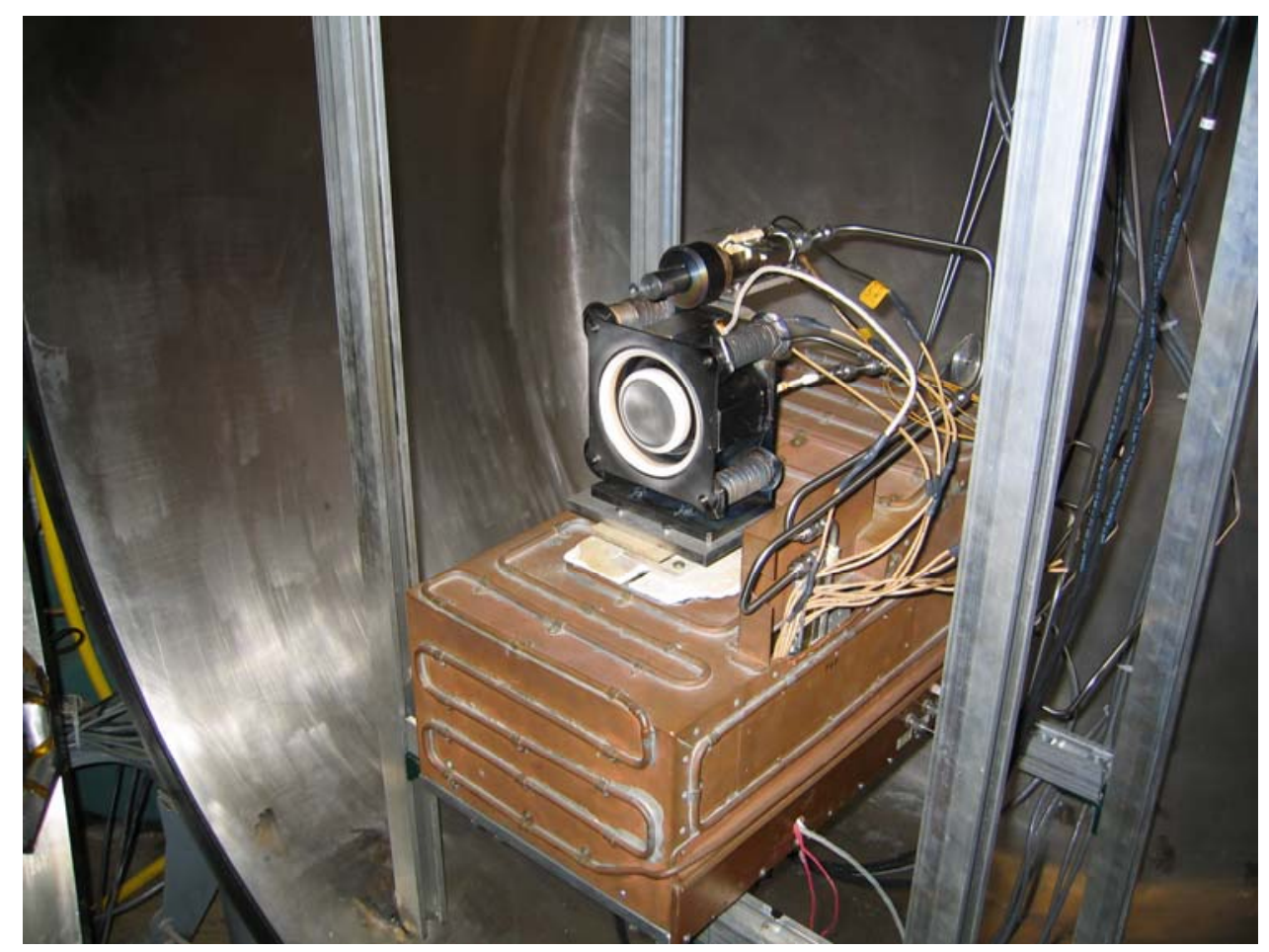

Figure 3-3: Photograph of a Hall thruster on the thrust stand 


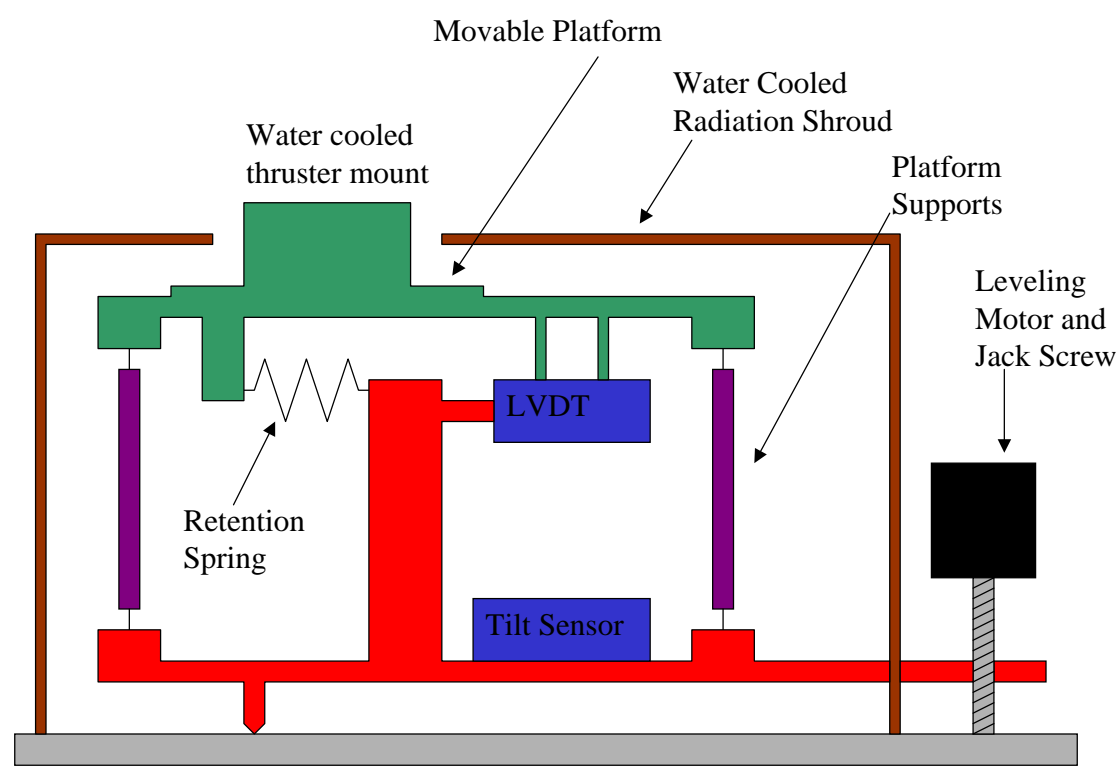

Figure 3-4: Inverted pendulum thrust stand. Green section is the movable platform which makes up the top of the inverted pendulum, violet is the tiltable supports held by leaf springs which support the movable platform, and red is the adjustable-tilt base.

Due to the unstable nature of the thrust measurement provided by an inverted pendulum, the thrust stand must be maintained at a precise angle relative to the horizontal plane. Any change in orientation angle from this best point will cause the thrust stand to gradually drift, as its null-force point is no longer facing directly upwards. Measurement of this angle is accomplished through the use of a high-precision tilt sensor, capable of 0.2 arc second resolution over a range of $+/-0.5$ degrees. The lower frame of the thrust stand was supported on the base plate in three places; two flexible posts at the front, which were threaded into the base plate and thus not able to move, and a threaded jack screw in the back. The jack screw was threaded into the thrust stand frame, with the bottom resting against the base plate. Adjustment of the screw would then raise or lower the back of the thrust stand, thus controlling the level. A computercontrolled stepper motor controlled of the screw. The resolution of the motor and the thread pitch of the screw were such that a single step of the motor translated to a 1.6-micron elevation change in the screw.

Given the distance between the front supports and the jack screw was approximately $0.5 \mathrm{~m}$, the change in angle was calculated to be 0.65 arc seconds per motor step, from the equation:

$$
\Delta \theta=\arcsin \left(1.6 \times 10^{-6} / 0.5\right)
$$


Calibration of the thrust stand is accomplished through the use of eight weights attached to a string, which is attached to a reel on an electric motor above the thrust stand as shown in Figure 3-5. Calibration is accomplished by turning the reel to shift the weights to pull on the thrust stand, adding a new weight for each measurement point in the calibration. As the masses (and thus, the weights) of the weights is known, the voltage output from the LVDT can be converted into a series of points which describe a linear relationship. Performing a linear regression on the calibration curve provides the slope and intercept necessary to obtain a thrust measurement from the thrust stand.

Thermal control of the thrust stand is highly important, as any change in temperature will change the stiffness of the leaf and retention springs, changing the calibration of the thrust stand. Control of temperature was accomplished by water-cooling the mounting block for the thruster, and enclosing the thrust stand in a water cooled copper shroud. The cooled mounting block prevented any

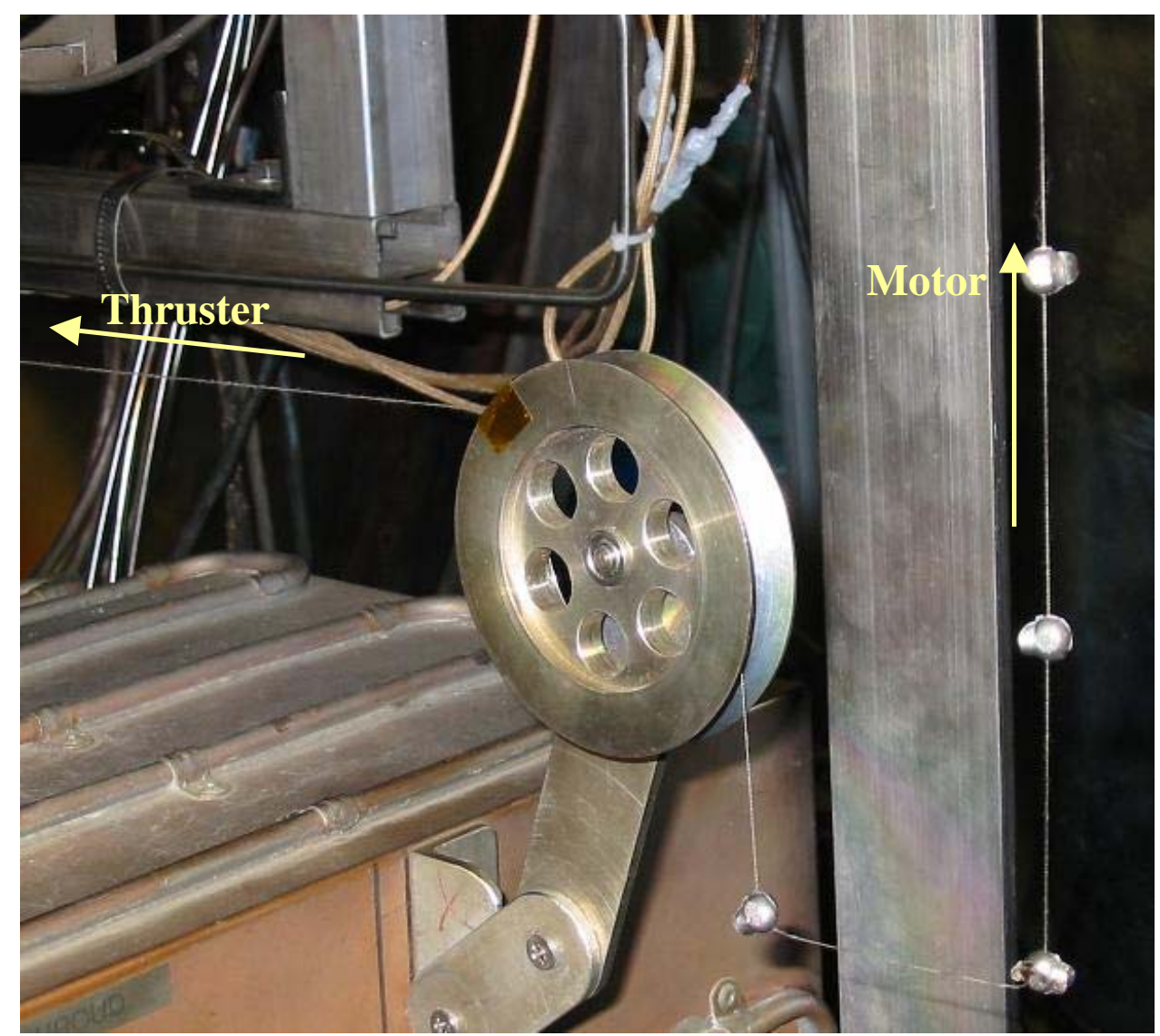

Figure 3-5: Photograph of the calibration system 
temperature change via conduction from the thruster, and the shroud prevented heating of the thrust stand via radiation. To ensure the thrust stand was at thermal equilibrium, the cooling water was turned on well in advance of the onset of testing, to allow several hours for the thrust stand to reach thermal equilibrium.

\subsection{Thruster Operating Characteristics}

Hall thrusters typically use the anode voltage, magnetic field, and propellant mass flow as control points. Discharge current is not actively regulated, and can rise or fall freely according to the three controlling factors. This is complicated somewhat in a segmented electrode Hall thruster. The voltage on the shims or the main anode needs to be adjusted to divert the discharge current from the main anode to the shims. In the experiments reported here, the shim voltage was used as the control for the shim current and hence, the discharge electron heating. A sample plot of the current on the shims and the main anode, as well as the total discharge current, is included in Figure 3-6. Additionally, the complete test matrix performed, with voltages and currents for every operating point examined are in Table 3-4.

There are two roughly linear regions in the current characteristic. The extremes of the characteristic show a roughly equal shallow slope, while the center of the characteristic has a much higher slope. This indicates that the change in voltage necessary to adjust the current attachment when it is equally shared between the main anode and the shims is much smaller than that required to force all of the current to either the shims or the main anode. This can complicate fine control of the level of discharge heating on either the shims or the main anode, as points in the center of the characteristic are very sensitive to small changes in voltage. An unexpected result of shifting all of the discharge current to the shims is that the voltage required to do so is higher than the anode voltage. This is a unique voltage configuration, and will be discussed in more detail in Chapter 7. 


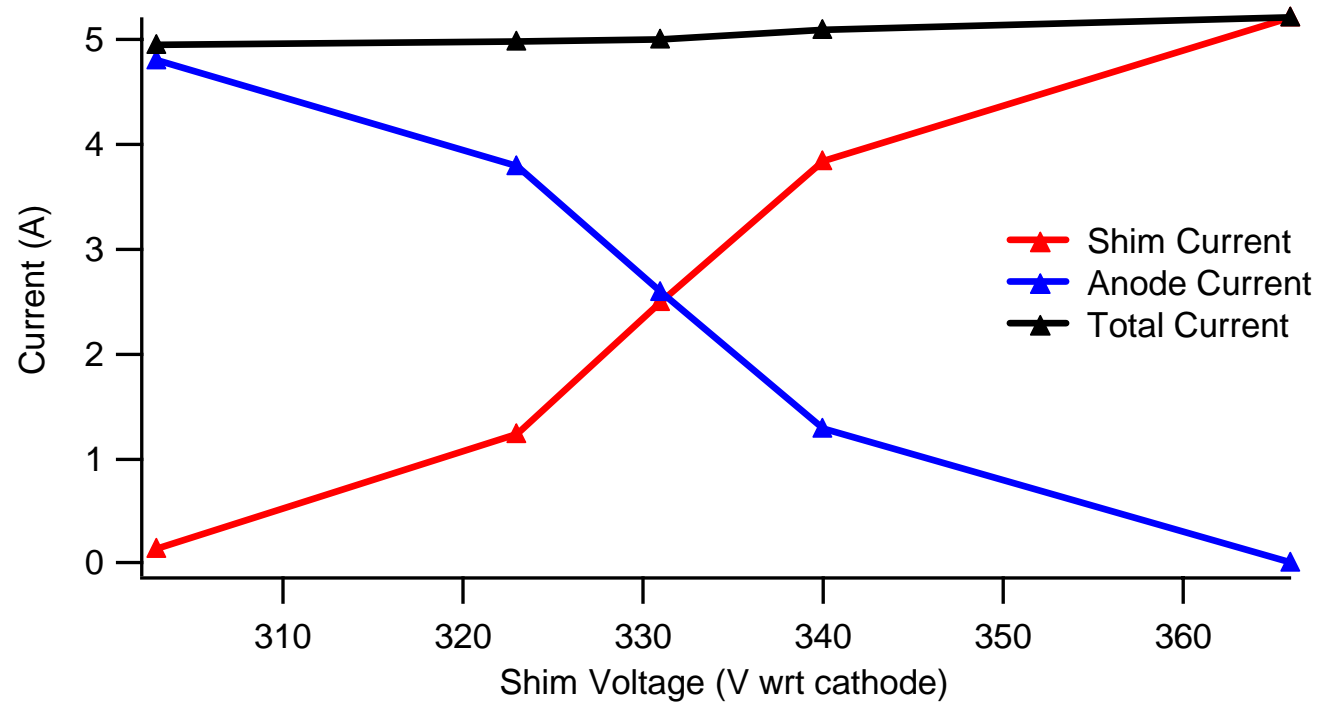

Figure 3-6: Characteristic shift in current on the shims and main anode as shim voltage was varied. Thruster was operating at $350 \mathrm{~V}$ on the anode and $5 \mathrm{mg} / \mathrm{s}$ of mass flow.

The other combinations of anode voltage and mass flow showed similar behavior, as shown for all anode voltages in Figure 3-7. At all combinations of mass flow and anode voltage, the initial increase as current was first moved to the shims was slow as a function of voltage, then increased rapidly through the operating points with $25 \%, 50 \%$, and $75 \%$ of the current on the shims, then slowed again as the current approached $100 \%$ on the shims.

The total current of the thruster was also slightly affected by the diversion of current from the shims to the main anode, as shown in Figure 3-8. The condensed x-axis shows the relationship of shim

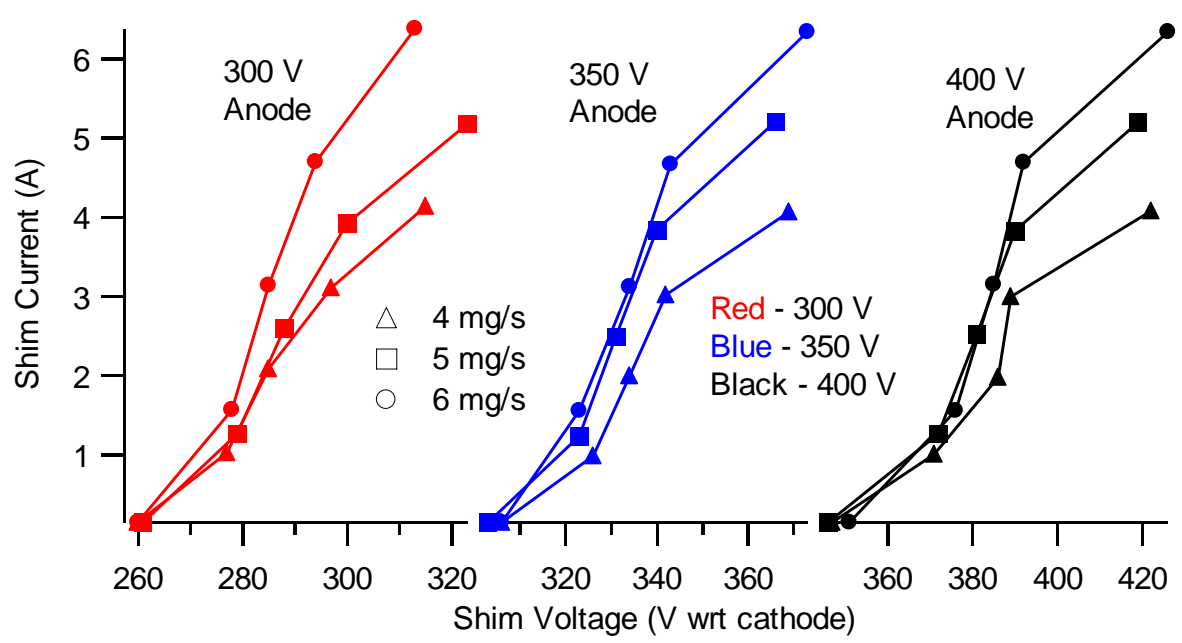

Figure 3-7: Shim Current as a function of shim voltage at all thruster operating points 


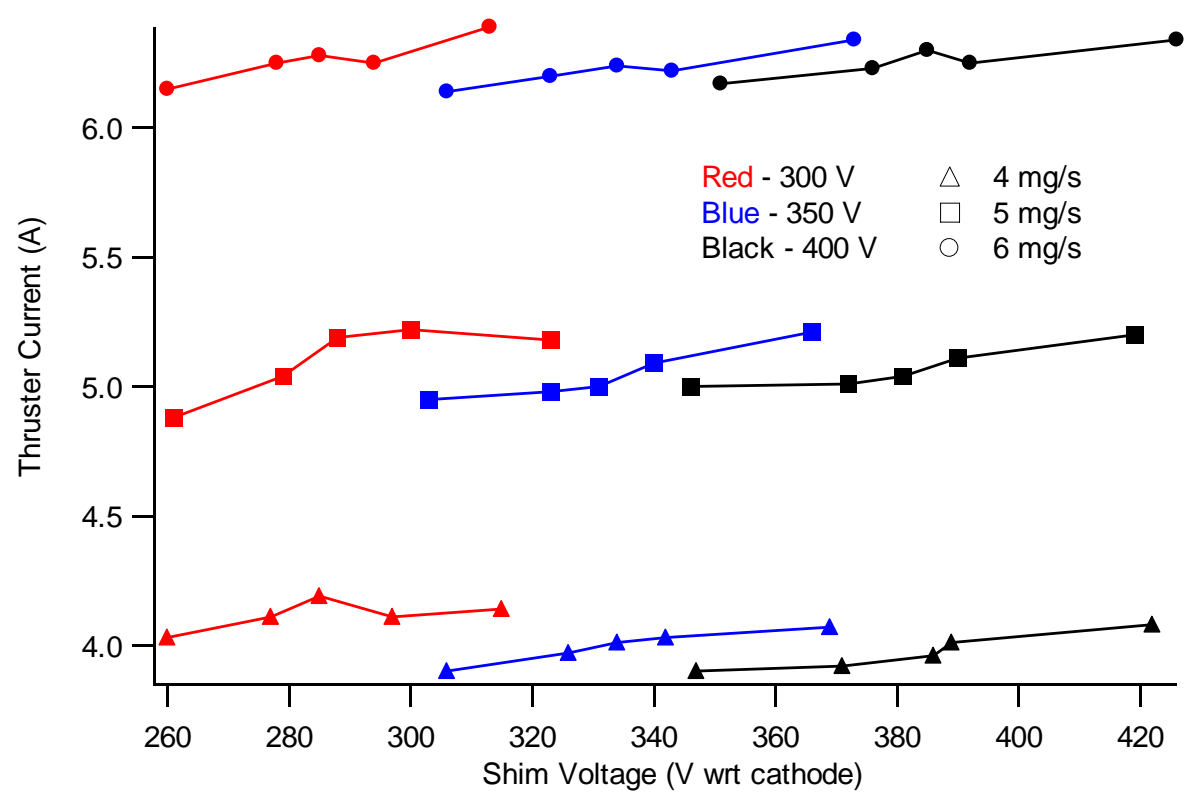

Figure 3-8: Total thruster current as a function of all three experimental factors

voltages among the different thruster operating points. At each combination of mass flow and anode voltage, the total current increased with shim voltage. In some cases the current reached a maximum when the current was evenly split between the anode and shims, but in the majority of cases the maximum was when all current was on the shims. A reason for this can be attributable to any number of reasons; increased ionization fraction of the propellant, increased multiply charged ion fraction, and/or increased electron mobility are the most likely sources of this change.

Total thruster power also changed with shim potential, as shown in Figure 3-9. The change in power does not have the same shape as the changes in current with shim voltage, rather at many of the combinations of anode voltage and mass flow the thruster power was minimized when $25 \%$ or $50 \%$ of the discharge current was on the shims and maximum when all discharge current was on the shims. The reason for maximum power when all current was on the shims is obvious; the combination of high total current and high shim voltage result in a higher total discharge power. The low power at $25 \%$ and $50 \%$ of the discharge current on the shims is due to two factors; first, the total current is lower, and second a significant percentage of current is on the shims, which are at a relatively low voltage. The subsequent rise in power as discharge current was shifted fully to the main anode is due to the anode voltage being higher than the shim voltage, thus the diverted current is being pulled through a higher potential and provides more power. 


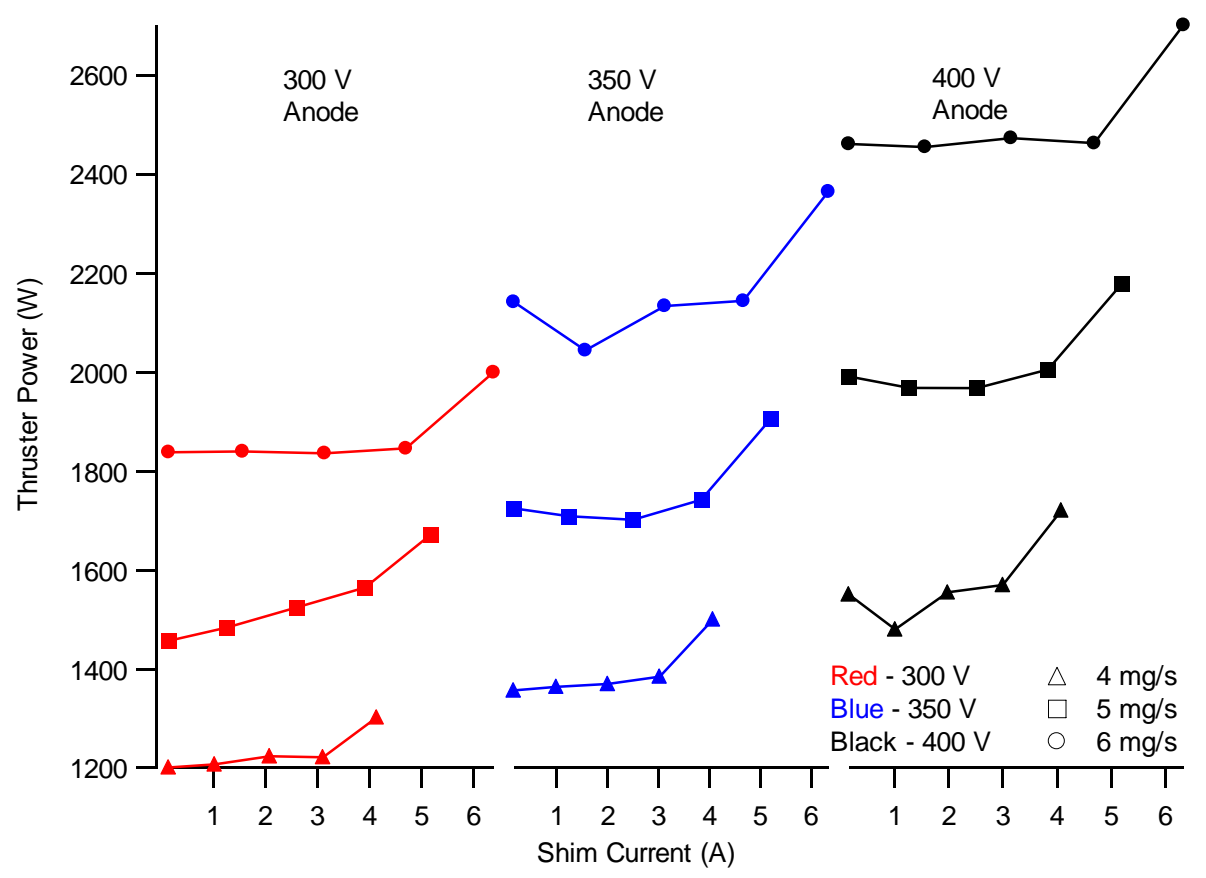

Figure 3-9: Discharge power as a function of all three experimental factors

\subsection{Thrust Characterization}

Thrust was measured directly by the thrust stand and recorded by a computer. Plotting the thrust measured for each anode voltage and mass flow combination as a function of shim voltage showed the general trends in thrust as all three factors were varied, as shown in Figure 3-10. The first two conclusions to be drawn from these data are that thrust increased with mass flow and with anode voltage. Both these conclusions are expected; an increase in anode voltage (and the corresponding increase in shim voltage required to divert electron heating to the desired location) will accelerate the ions to higher velocities than a low voltage, and hence, increase the thrust force. Increased mass flow will also directly increase the thrust force, assuming the ion acceleration mechanism (and hence, ion velocity) is unaffected. Additionally, the thrust appears to increase with shim voltage, a trend which indicates ion acceleration is dictated at least somewhat by the shim voltage. 


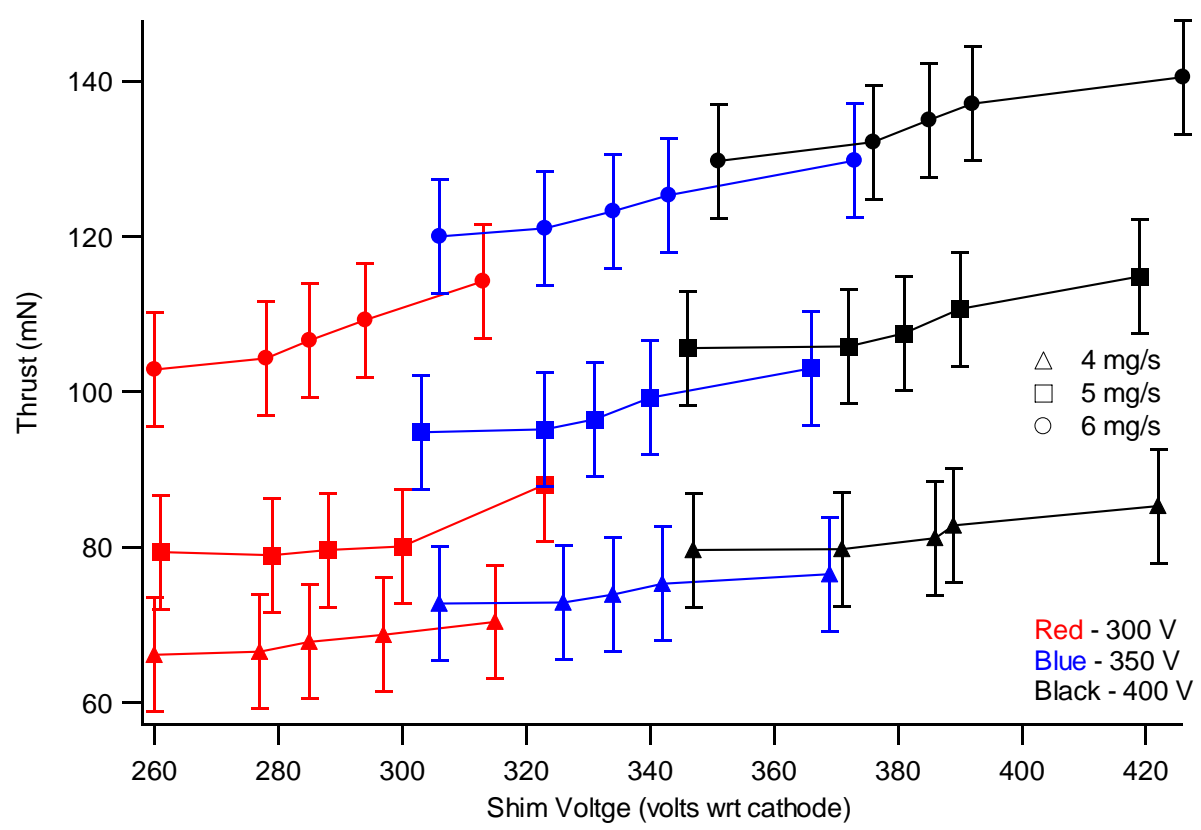

Figure 3-10: Thrust measured as a function of all three experimental factors. Higher shim voltages for a given mass flow and anode voltage combination correspond to higher discharge heating acceptance by the shims.

Application of the analysis of variance as outlined in Appendix B to these data show that all three experimental factors have an effect on the thrust, with excellent certainty, as shown in Table 3-1. The Pvalues estimate the probability that the null hypothesis is true. As each of them is well below one percent, it can be said with excellent certainty that shifting discharge electron heating from the anode to the shims has a net effect on the thrust.

The width of the $95 \%$ confidence interval on the thrust data, then, is calculated to be $6.68 \mathrm{mN}$. This is a moderate uncertainty, ranging from $5-10 \%$ of the measured values of thrust. This uncertainty could be greatly reduced if further testing of the thruster was performed, allowing a reduction in the t-distribution value used as well as a reduced contribution from the sample variance.

Table 3-1: ANOVA table of Thrust

\begin{tabular}{|c|c|c|c|c|c|}
\hline Factor & Sum of Squares & $\begin{array}{c}\text { Degrees of } \\
\text { Freedom }\end{array}$ & Mean Squared & $\mathrm{F}_{0}$ & P-Value \\
\hline Anode Voltage & 3978.7 & 2 & 1989.3 & 183.3 & $1.3 \times 10^{-19}$ \\
\hline Mass Flow & 16909.1 & 2 & 8454.6 & 779.2 & $2.3 \times 10^{-30}$ \\
\hline Shim Level & 370.2 & 4 & 92.6 & 8.5 & $6.0 \times 10^{-5}$ \\
\hline Error & 390.6 & 36 & 10.9 & & \\
\hline Total & 21648.6 & 44 & & & \\
\hline
\end{tabular}




\subsection{Specific Impulse Characterization}

Specific impulse of the thruster at all operating points is plotted in Figure 3-11. The trends in specific impulse are similar to thrust; specific impulse appeared to increase with all three factors. A notable exception to this is the specific impulse at $300 \mathrm{~V}$ on the main anode and $5 \mathrm{mg} / \mathrm{s}$ of mass flow. In this case the specific impulse was lower than that seen at $300 \mathrm{~V}$ and $4 \mathrm{mg} / \mathrm{s}$, the opposite of the trend seen at the other operating points.

Analysis of variance shows that, similarly to thrust, the specific impulse sees a significant effect from all three factors, as shown in Table 3-2. The P-values for each factor are extremely small, similarly to the thrust measurement, however the relationship of P-values to each other is slightly different. While in the thrust measurements the smallest P-value was for mass flow (thus indicating that mass flow was the most significant factor), the most significant factor in specific impulse was the anode voltage. In specific impulse, as in thrust, the shim discharge heating level was the least-important factor.

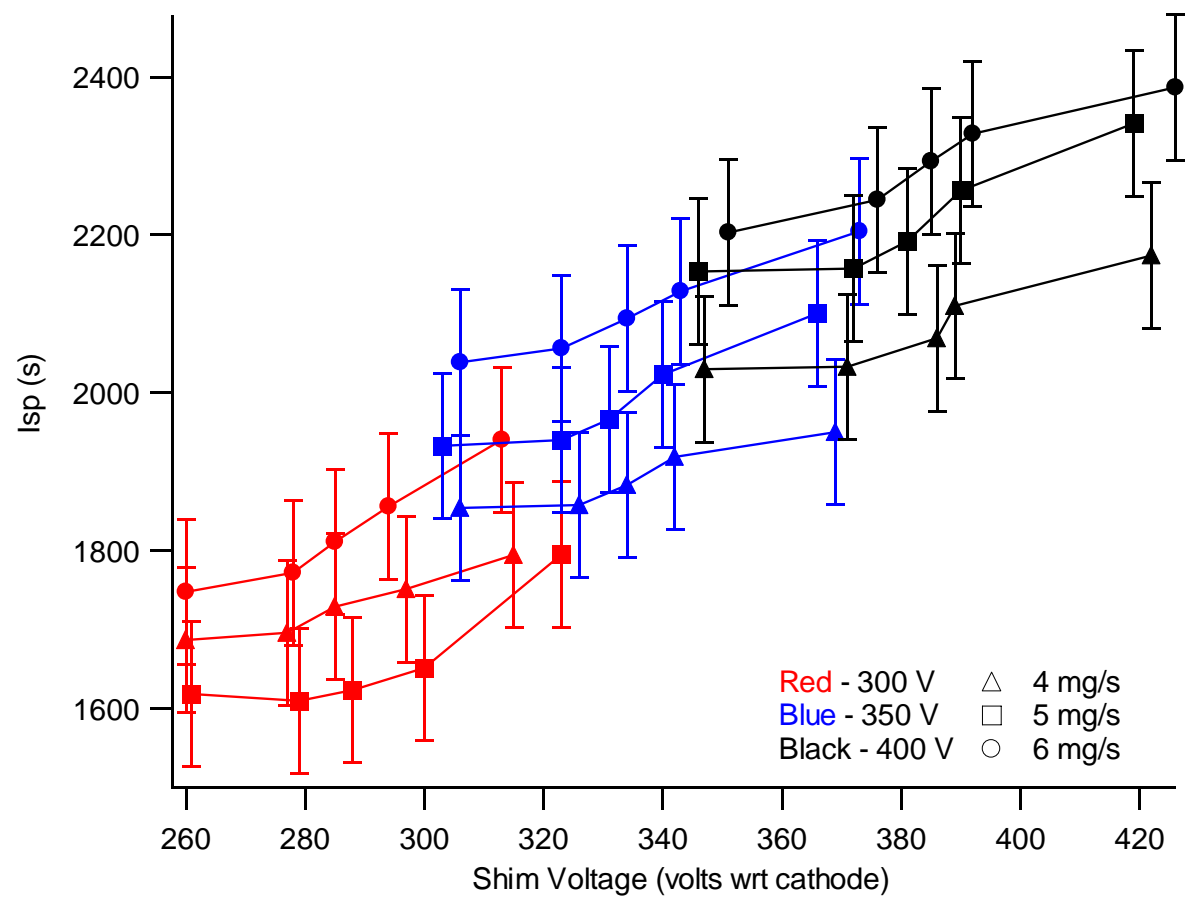

Figure 3-11: Specific Impulse as a function of shim voltage for all thruster operating conditions tested. Higher shim voltage for a given anode voltage indicates higher discharge electron heating on the shims. 
Table 3-2: ANOVA table of Specific Impulse

\begin{tabular}{|c|c|c|c|c|c|}
\hline Factor & Sum of Squares & $\begin{array}{c}\text { Degrees of } \\
\text { Freedom }\end{array}$ & Mean Squared & $\mathrm{F}_{0}$ & P-value \\
\hline Anode voltage & 1589512 & 2 & 794756 & 383.8 & $5.3 \times 10-25$ \\
\hline Mass flow & 229040 & 2 & 114520 & 55.3 & $1.1 \times 10-11$ \\
\hline Shim Level & 147821 & 4 & 36955 & 17.8 & $3.7 \times 10-8$ \\
\hline Error & 74539 & 36 & 2071 & & \\
\hline Total & 2040912 & 44 & & & \\
\hline
\end{tabular}

The 95\% confidence interval on the data is somewhat smaller for specific impulse than for thrust; the width of the interval is $92.3 \mathrm{~s}$, only $3.8-5.7 \%$ of the measurement. While this error is smaller as a percentage of the measurement than that for thrust, the uncertainty is still on the order of the specific impulse change over the range of shim sharing levels.

\subsection{Efficiency Characterization}

Efficiency is a direct measure of energy wasted during thruster operation. It is therefore important to spacecraft designers who desire the smallest possible power input (and thus the smallest possible power supply) for a given amount of thrust. Efficiency was calculated from the power and propellant mass flow supplied to the thruster and the thrust output. Efficiency for all thruster operating points is plotted in Figure 3-12. Similarly to thrust and specific impulse, the data indicate a significant dependence on anode voltage and mass flow. The increase in thruster efficiency with thruster voltage was expected, and has been seen in previous thruster investigations. ${ }^{21,23}$ Unlike thrust and specific impulse, however, the efficiency change with shim voltage does not appear to indicate that there is a significant effect on the efficiency as a function of shim voltage.

Examining the efficiency data through use of the analysis of variance in Table 3-3 shows that the conclusions reached by initial visual inspection are correct. Thruster efficiency is significantly affected by both thrust and propellant mass flow. The P-value of 0.522 for the discharge electron heating level, which corresponds to a $52.2 \%$ certainty that the null hypothesis is true, is large enough that it can be said with good confidence that the location of discharge electron heating does not significantly affect the efficiency of the thruster. 


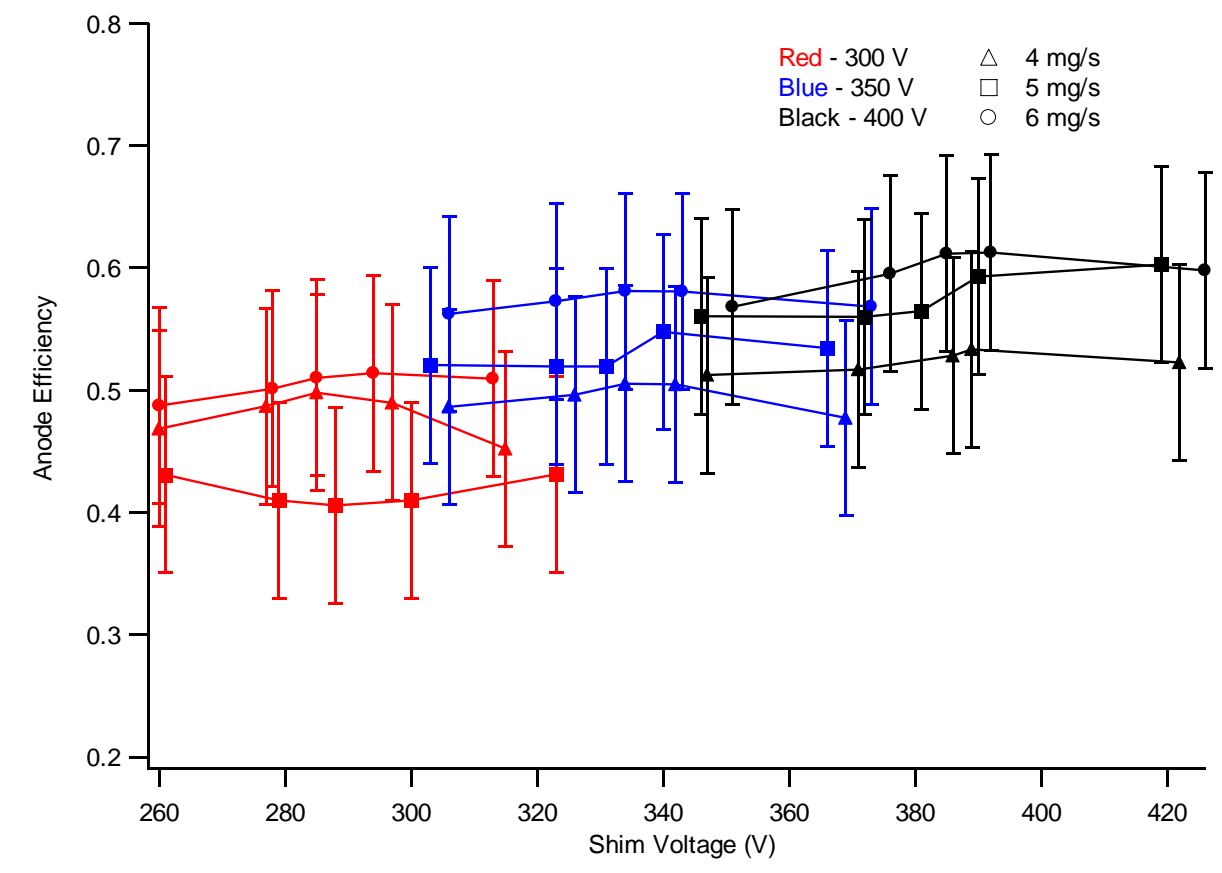

Figure 3-12: Thruster Efficiency as a function of all three experimental factors. Higher shim voltage for a given anode voltage indicates higher discharge heating on the shims

Table 3-3: ANOVA table of Efficiency

\begin{tabular}{|c|c|c|c|c|c|}
\hline Factor & Sum of Squares & $\begin{array}{c}\text { Degrees of } \\
\text { Freedom }\end{array}$ & Mean Squared & $\mathrm{F}_{0}$ & P-value \\
\hline Voltage & .07494 & 2 & .03747 & 55.72 & $9.515 \times 10-12$ \\
\hline Mass Flow & .03108 & 2 & .01554 & 23.11 & $3.499 \times 10-7$ \\
\hline Shim Level & .0022 & 4 & .00055 & .8183 & .522 \\
\hline Error & .02 & 36 & .00067 & & \\
\hline Total & .13 & 44 & & & \\
\hline
\end{tabular}

Application of the estimate of variance obtained by the analysis of variance, a confidence interval can be constructed of the thruster efficiency, using the same method as for thrust and specific impulse. The 95\% confidence interval on efficiency has a width of .0526 . This uncertainty is large relative to the measurements themselves; at best, the uncertainty is $8.6 \%$ of the measurement, and at worst it is $13 \%$ of the measurement; a much larger uncertainty than that seen in the specific impulse or thrust data. 
Table 3-4: Thruster operating conditions examined

\begin{tabular}{|c|c|c|c|c|c|c|}
\hline $\begin{array}{l}\text { Anode } \\
\text { Voltage }\end{array}$ & $\begin{array}{l}\text { Anode } \\
\text { Current }\end{array}$ & $\begin{array}{c}\text { Shim } \\
\text { Voltage }\end{array}$ & $\begin{array}{c}\text { Shim } \\
\text { Current }\end{array}$ & $\begin{array}{c}\text { Total } \\
\text { Current }\end{array}$ & $\begin{array}{c}\text { Total } \\
\text { Power }\end{array}$ & $\begin{array}{c}\text { Operating Point } \\
\text { Label }\end{array}$ \\
\hline \multicolumn{7}{|c|}{$4 \mathrm{mg} / \mathrm{s}$ of xenon } \\
\hline \multirow{5}{*}{$300 \mathrm{~V}$} & 0 & 323 & 4.14 & 4.14 & 1304.1 & 304100 \\
\hline & 1 & 300 & 3.11 & 4.11 & 1223.7 & 30475 \\
\hline & 2.1 & 288 & 2.09 & 4.19 & 1225.7 & 30450 \\
\hline & 3.08 & 279 & 1.03 & 4.11 & 1209.3 & 30425 \\
\hline & 3.88 & 261 & .15 & 4.03 & 1203 & 3040 \\
\hline \multirow{5}{*}{$350 \mathrm{~V}$} & 0 & 366 & 4.07 & 4.07 & 1501.8 & 354100 \\
\hline & 1.01 & 340 & 3.02 & 4.03 & 1386.3 & 35475 \\
\hline & 2.01 & 331 & 2 & 4.01 & 1371.5 & 35450 \\
\hline & 2.98 & 323 & .99 & 3.97 & 1365.7 & 35425 \\
\hline & 3.75 & 303 & .15 & 3.9 & 1356.4 & 3540 \\
\hline \multirow{5}{*}{$400 \mathrm{~V}$} & 0 & 419 & 4.08 & 4.08 & 1321.8 & 404100 \\
\hline & 1.01 & 390 & 3 & 4.01 & 1571 & 40475 \\
\hline & 1.98 & 381 & 1.98 & 3.96 & 1556.3 & 40450 \\
\hline & 2.98 & 372 & .94 & 3.92 & 1481.6 & 40425 \\
\hline & 3.75 & 346 & .15 & 3.9 & 1552.1 & 4040 \\
\hline \multicolumn{7}{|c|}{$5 \mathrm{mg} / \mathrm{s}$ of xenon } \\
\hline \multirow{5}{*}{$300 \mathrm{~V}$} & 0 & 323 & 5.18 & 5.18 & 1673.1 & 305100 \\
\hline & 1.3 & 300 & 3.92 & 5.22 & 1566 & 30575 \\
\hline & 2.59 & 288 & 2.6 & 5.19 & 1525.8 & 30550 \\
\hline & 3.77 & 279 & 1.27 & 5.04 & 1485.3 & 30525 \\
\hline & 4.73 & 261 & .15 & 4.88 & 1458.2 & 3050 \\
\hline \multirow{5}{*}{$350 \mathrm{~V}$} & 0 & 366 & 5.21 & 5.21 & 1906.9 & 355100 \\
\hline & 1.25 & 340 & 3.84 & 5.08 & 1743.1 & 35575 \\
\hline & 2.5 & 331 & 2.5 & 5 & 1702.5 & 35550 \\
\hline & 3.74 & 323 & 1.24 & 4.98 & 1709.5 & 35525 \\
\hline & 4.8 & 303 & .15 & 4.95 & 1725.5 & 3550 \\
\hline \multirow{5}{*}{$400 \mathrm{~V}$} & 0 & 419 & 5.2 & 5.2 & 2178.8 & 405100 \\
\hline & 1.28 & 390 & 3.83 & 5.11 & 2005.7 & 40575 \\
\hline & 2.52 & 381 & 3.53 & 5.04 & 1968.1 & 40550 \\
\hline & 3.74 & 372 & 1.27 & 5.01 & 1968.4 & 40525 \\
\hline & 4.85 & 346 & .15 & 5 & 1991.9 & 4050 \\
\hline \multicolumn{7}{|c|}{$6 \mathrm{mg} / \mathrm{s}$ of xenon } \\
\hline \multirow{5}{*}{$300 \mathrm{~V}$} & .01 & 313 & 6.38 & 6.39 & 1999.9 & 306100 \\
\hline & 1.55 & 294 & 4.7 & 6.25 & 1846.8 & 30675 \\
\hline & 3.14 & 285 & 3.14 & 6.28 & 1836.9 & 30650 \\
\hline & 4.68 & 278 & 1.57 & 6.25 & 1840.5 & 30625 \\
\hline & 6 & 260 & .15 & 6.15 & 1839 & 3060 \\
\hline \multirow{5}{*}{$350 \mathrm{~V}$} & 0 & 373 & 6.34 & 6.34 & 2364.8 & 356100 \\
\hline & 1.55 & 343 & 4.67 & 6.22 & 2144.3 & 35675 \\
\hline & 3.12 & 334 & 3.12 & 6.24 & 2134.1 & 35650 \\
\hline & 4.65 & 323 & 1.55 & 6.2 & 2044.5 & 35625 \\
\hline & 5.99 & 306 & .15 & 6.14 & 2142.4 & 3560 \\
\hline \multirow{4}{*}{$400 \mathrm{~V}$} & 0 & 426 & 6.34 & 6.34 & 2700.8 & 406100 \\
\hline & 1.56 & 392 & 4.69 & 6.25 & 2462.5 & 40675 \\
\hline & 3.15 & 385 & 3.15 & 6.3 & 2472.8 & 40650 \\
\hline & 4.67 & 376 & 1.56 & 6.23 & 2454.6 & 40625 \\
\hline
\end{tabular}




\section{Chapter 4: Plume Divergence Characterization}

Characterization of the plume divergence of the thruster may provide insight into the changes seen in performance as the discharge current is shifted from the main anode to the shims. There are two ways in which beam divergence is an important factor in thruster design; loss of performance and spacecraft integration issues. The aspect of beam divergence which is more important here is the effect on performance. It has already been established that the performance of the thruster changes as the location of discharge electron heating changes from the shims to the main anode, so the source of these changes must be determined. Any ions leaving the thruster with velocities at nonzero angles relative to the thrust axis will contribute less to thrust than ions accelerated directly along the axis. This is due to the fact that an ion accelerated at a nonzero angle relative to the thrust axis has two components of velocity; one along the thrust axis and one perpendicular to it (assuming a cylindrical coordinate system, the axial component would be in the $z$-direction and the off-axis component would be in the $r$-direction). Assuming the thruster is axisymmetric, any ion accelerated one direction relative to the thrust axis has a counter part accelerated at an equal angle off axis in the opposite direction. Thus the perpendicular components of thrust are canceled out, leaving only the axial component. As the axial component of velocity (and hence, thrust) is dictated by $v_{z}=v \cos \theta$, the total thrust of an ion accelerated at a nonzero angle from the axis of thrust will contribute less to thrust than one accelerated parallel to the axis. Spacecraft integration issues arise as anything placed within the plume of the thruster will be damaged by the plume ions, thus reducing its lifetime. Hall thrusters have significant plume divergence angles which are of major concern for spacecraft integration and design. With the addition of segmented electrodes, any change in beam divergence as discharge electron heating is moved between the shims and the anode can further complicate the design issues presented by the Hall thruster plume.

\subsection{Faraday Probe}

A Faraday probe is a planar electrostatic probe, ${ }^{24,25,26}$ biased negative relative to the plasma floating potential in the ion saturation regime. Biasing to this voltage allows the probe to pull in the ions in the discharge, while rejecting discharge electrons. Data are acquired by sweeping the probe across the face 
of the thruster, and measuring the current collected at each point in the sweep. Faraday probe sweeps are usually circular arcs at a constant radius from the thruster, allowing the current measurements to represent the relative flux of ions as a function of distance or angle from the thruster axis. Distance from the thruster has a large effect on ion density. Eliminating this as a factor in the ion current measurement allows determination of the plume divergence. Faraday probes have seen extensive use in characterization of Hall thruster plumes in previous studies ${ }^{16,17,18,19,20,21,24,25}$

Typical Faraday probes are constructed from a flat electrode surrounded by a conductive guard ring. The electrode and guard ring are electrically isolated and biased to the same potential. This allows for a flatter electric field profile directly in front of the probe, and thus prevents the probe from pulling in ions from the sides of its scan area, keeping the collected current density as accurate as possible. Ion current density is calculated by simply dividing the probe current by the area of the probe.

The Faraday probe utilized in the work reported here consisted of a 2.4-mm-dia. tungsten electrode enclosed in an alumina tube with diameter $4 \mathrm{~mm}$. A photograph of the probe used is included in Figure 4-1. The guard ring was constructed of stainless steel and surrounded the alumina tube at the end of the probe. Measurements were taken using a Keithley 2410 sourcemeter. Performing an I-V sweep and finding the voltage where the probe was fully in the ion saturation regime determined the bias voltage. The guard ring was operated on a separate power supply, set to the same voltage as the sourcemeter. Faraday probe scans were performed at $250 \mathrm{~mm}$ from the thruster exit, sweeping through off-axis distances of 200 $\mathrm{mm}$ on either side of the thruster axis. These off-axis distances corresponded to an off-axis angle of 53.1 degrees. The sweeps were divided into 51 equally-spaced points 2.124 degrees apart, such that the distance between points in the sweep was $9.3 \mathrm{~mm}$. Two measurements were made at every point in the probe sweep, allowing for determination of the sample variance of the current measurement. 


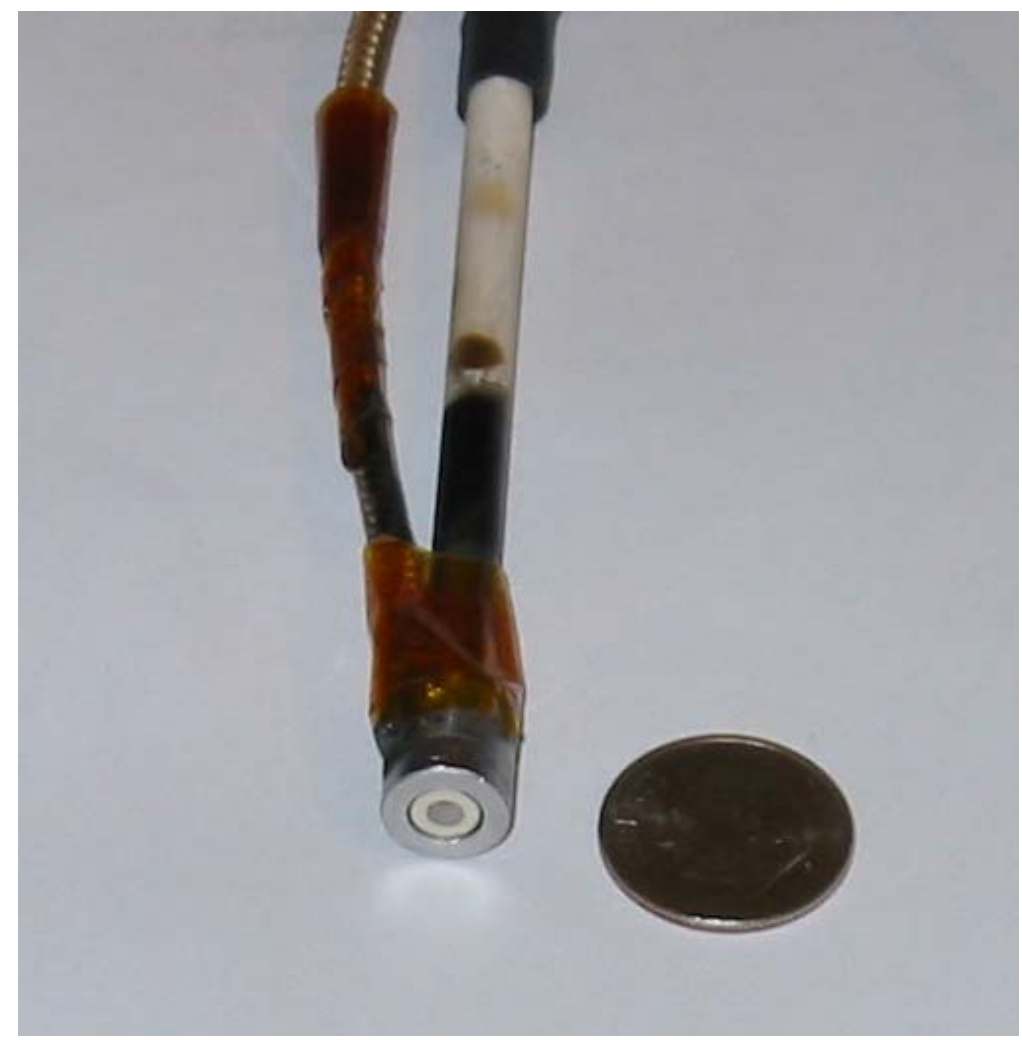

Figure 4-1: Photograph of the Faraday probe.

\subsection{Faraday Probe Sweep Data}

The sweeps taken by the Faraday probe are plotted in Figures 4-2 - 4-4. Several observations can be immediately made from these data: the ion current density is dependent on propellant mass flow in most cases and the level of discharge electron current on the shims, and that the thrust vector appears to change slightly as the discharge current is shifted between the main anode and the shims.

The first observation is that the collected ion current density is directly affected by the propellant mass flow in the thruster; in the majority of cases the current density increased with propellant mass flow. This was an expected change, as higher propellant flows should result in more ion production and thus more ion current in the plume of the thruster. An anomalous result is seen in the data when $300 \mathrm{~V}$ was on the anode, however. In the $25 \%$ and $<5 \%$ levels of discharge electron heating on the shims, the peak current density with $5 \mathrm{mg} / \mathrm{s}$ of mass flow was on the order of, or smaller than, the peak density at $4 \mathrm{mg} / \mathrm{s}$. This coincides with some of the performance measurements taken of the thruster at these operating points. A beam with poor focusing, as appears to be the case in the 305 set of operating conditions (as seen in 
Table 3-4) would exhibit lower thrust, specific impulse, and efficiency than a thruster with a wellcollimated beam.

It also can be seen from the data that the ion current density is dependent on the shim voltage. At all combinations of anode voltage and mass flow, the peak ion current density increased with increasing shim voltage, and thus increasing levels of discharge electron heating on the shims. 


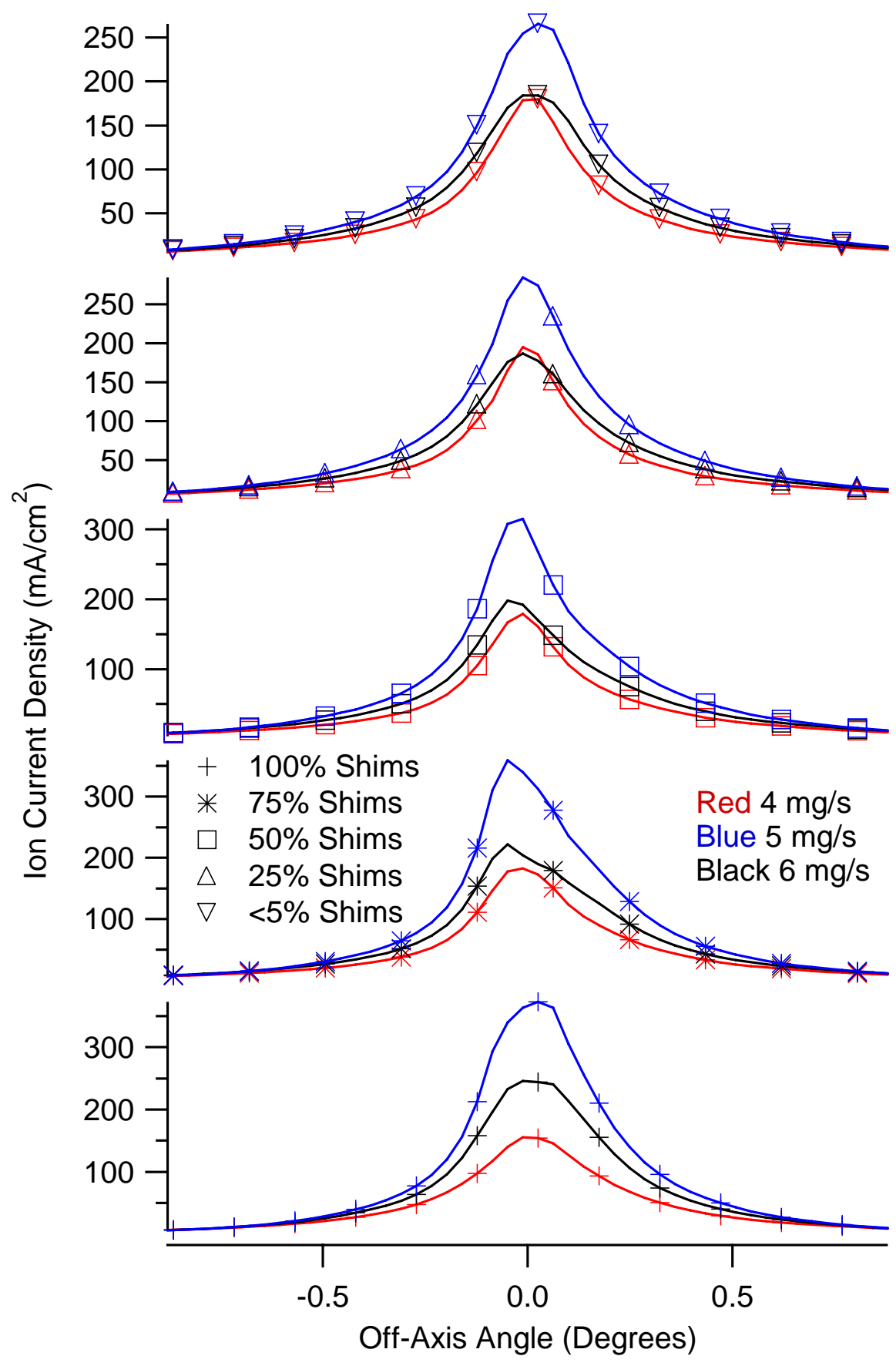

Figure 4-2: Beam Profiles with $300 \mathrm{~V}$ on the anode at all mass flows and levels of discharge current heating 


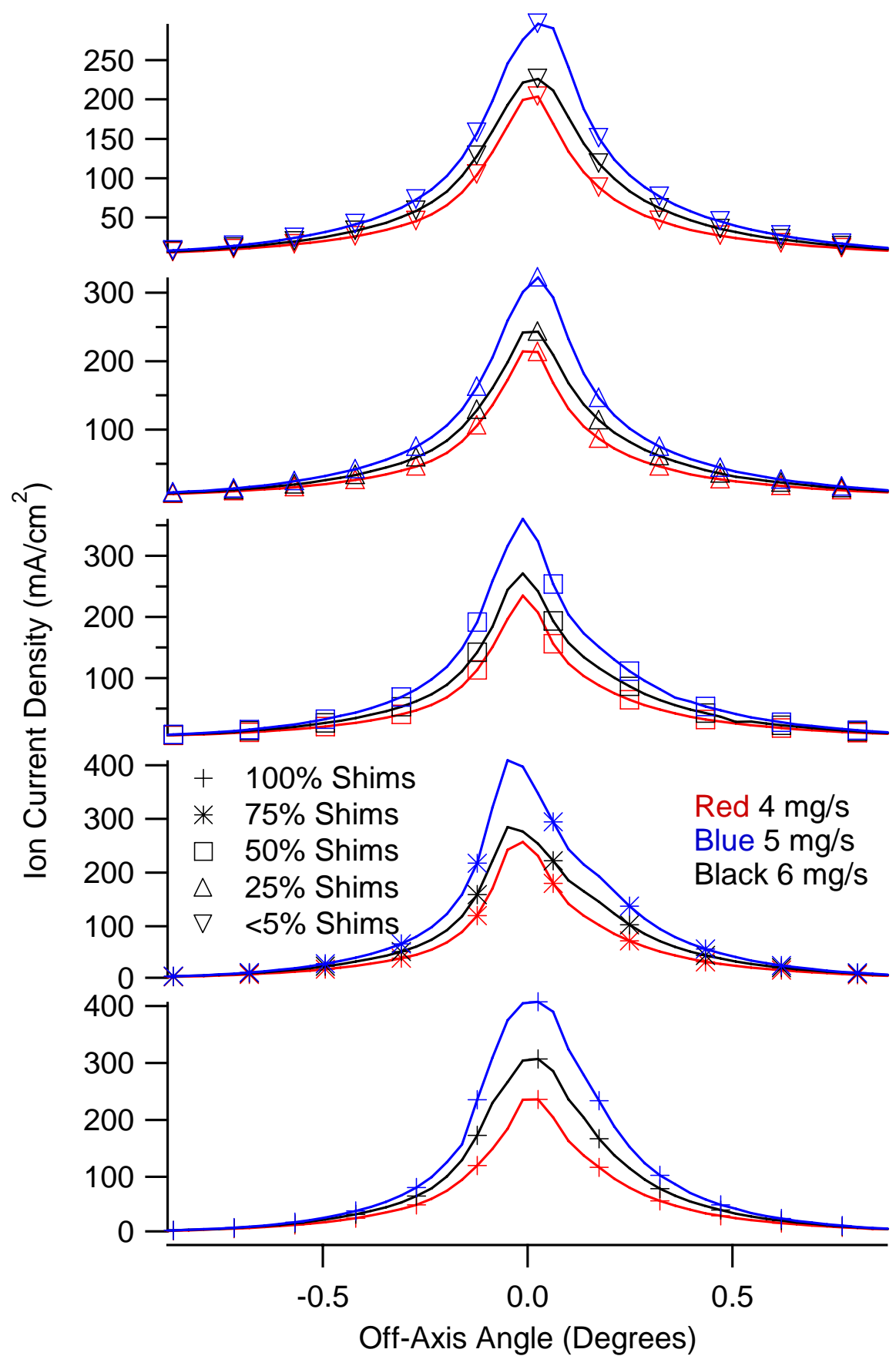

Figure 4-3: Beam Profiles with $350 \mathrm{~V}$ on the anode at all mass flows and levels of discharge current heating 


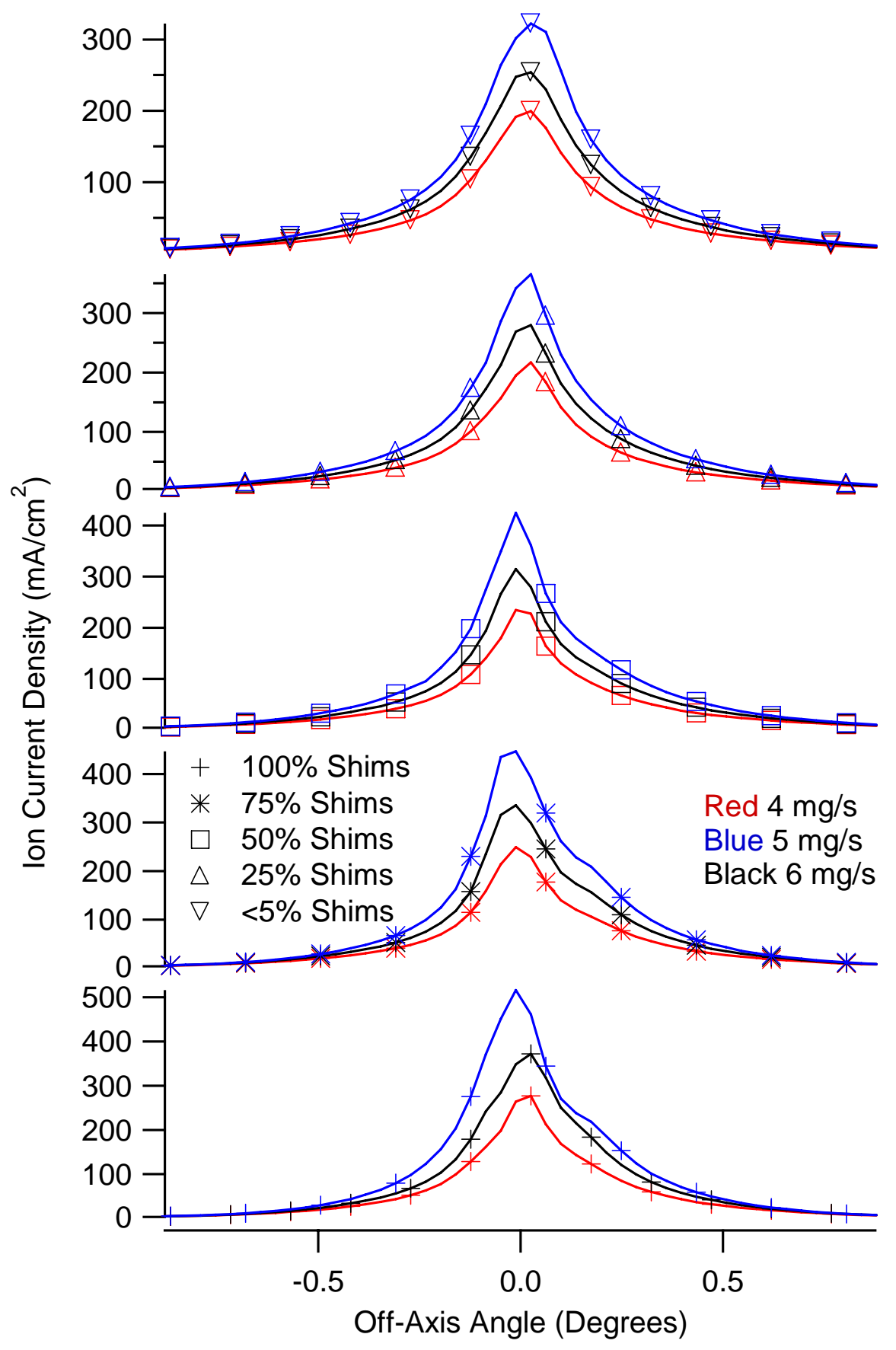

Figure 4-4: Beam Profile $s$ with $400 \mathrm{~V}$ on the anode at all mass flows and levels of discharge current heating 


\subsection{Beam divergence analysis}

Determination of the beam divergence can be accomplished through integration of the ion current density. Using a point along the axis and at the exit plane of the thruster as the zero point, an integration in spherical coordinates can be performed, which will yield the total beam current. Integration was performed using the following equation:

$$
I=\int_{-\theta_{m}}^{\theta_{m}} \int_{0}^{\pi} J_{i} r^{2} \sin \Theta d \varphi d \Theta
$$

The bound on the integration in $\Theta$ is due to the current measurements being taken at up to two equal angles off-axis of the thruster. Due to the double-sided nature of the integration in $\Theta$, the integration in $\varphi$ was performed between zero and $\pi$. $J_{i}$ is only a function of $\Theta$, so the double integral can be simplified to a single integral:

$$
I=\pi r^{2} \int_{-\theta_{m}}^{\theta_{m}} J_{i} \sin \Theta d \Theta .
$$

As both $\Theta$ and $J_{i}$ are discrete, the integral was solved numerically using the trapezoid method. The beam divergence was calculated by determining the half-angle required to include $90 \%$ of the total beam current. The results of this calculation are plotted in Figure 4-5. In nearly all of the data traces the beam divergence was lowest when all discharge electron heating was on the shims and highest when $25 \%$ of the discharge heating was on the shims. There were three notable discrepancies to this; in the 404-, 406-, and 306-set of operating conditions the divergence remained the same or slightly decreased as the discharge electron heating was shifted from $25 \%$ on the shims to $<5 \%$ on the shims. Additionally, the 404 -set of operating conditions showed a much higher than expected result at $100 \%$ of the discharge heating on the shims. The beam divergence also appeared to be dependent on thruster voltage and propellant mass flow. Increasing voltage and mass flow both appeared to contribute to a lower beam divergence.

Analysis of the calculated beam divergence through analysis of variance showed that as expected from the data, all three experimental factors contributed to the beam divergence, as shown in Table 4-1. All three experimental factors had a statistically significant effect on the beam divergence, as shown by the P-values. Additionally, the width of the 95\% confidence interval was 1.01 degrees, 


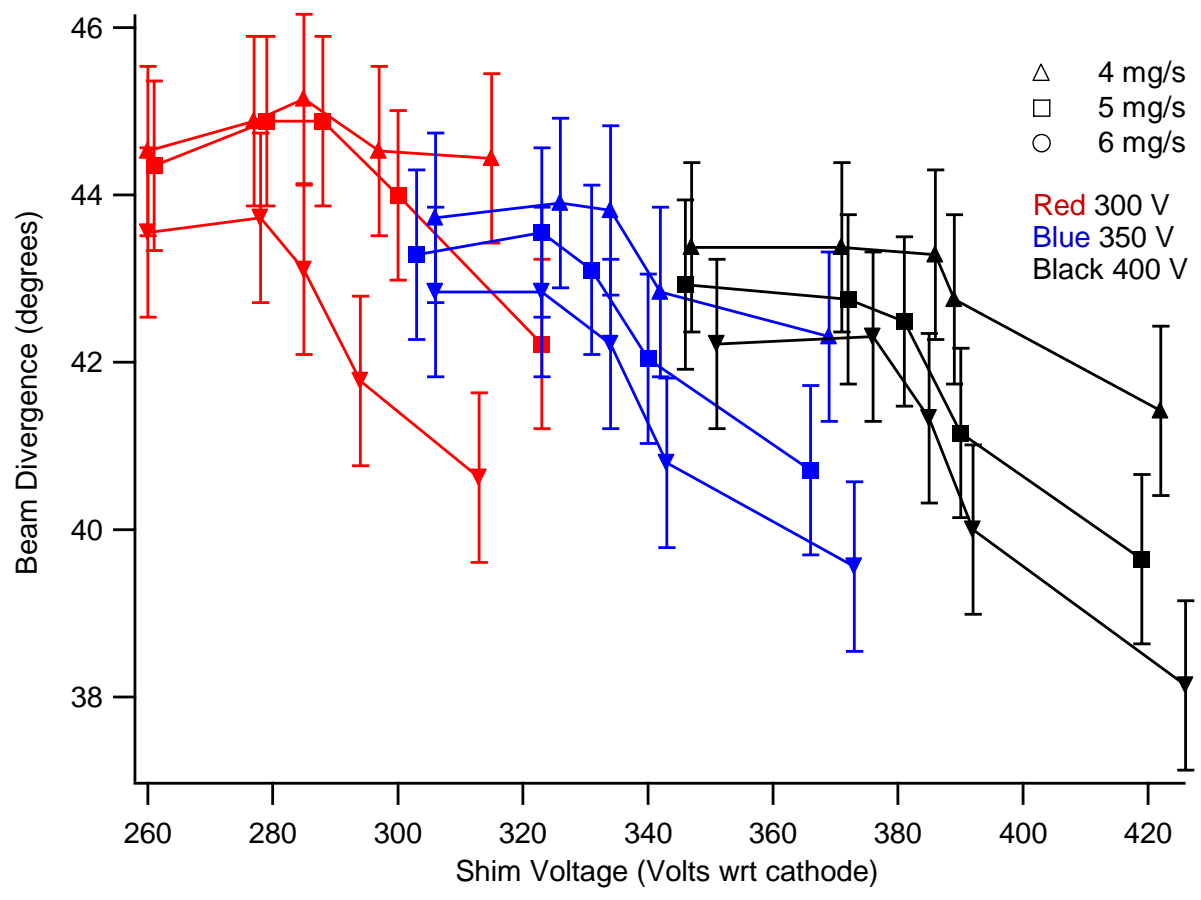

Figure 4-5: Beam divergence as a function of all three experimental factors

roughly one quarter of the total change in beam divergence caused by the shift of current from the shims to the main anode. An interesting result, however, is that the effect from the level of discharge current sharing on the shims has the most significant impact on the beam divergence. This can be seen in the plot of beam divergence; the shim voltage (and thus, amount of discharge heating on the shims) caused beam divergence to change by up to four degrees, while voltage or mass flow only accounted for two degrees at most. Thus the shims appear to have a focusing effect, forcing the ion beam to be more collimated with high levels of discharge heating on the shims. This is a significant change in beam divergence, as much as a $10 \%$ reduction as discharge electron heating is shifted from the main anode to the shims, so use of shim electrodes in a configuration similar to that examined here will reduce the beam divergence in potential flight applications. This is not completely unexpected, as the Princeton Plasma Physics Laboratory saw similar results with the addition of segmented electrodes for beam divergence control. ${ }^{17,18,19}$ However, as the nature of the operation of a bismuth thruster requires that the level of discharge electron heating be constantly modified, and also that it will be very rare that all discharge electron heating is on the shims, use of the highest value of beam divergence seen should be used in any spacecraft interaction analysis, as the thruster will likely need to operate at that point at some time during a mission. 
Table 4-1: ANOVA table of thruster beam divergence

\begin{tabular}{|c|c|c|c|c|c|}
\hline Factor & Sum of Squares & $\begin{array}{c}\text { Degrees of } \\
\text { Freedom }\end{array}$ & Mean Squared & $\mathrm{F}_{0}$ & P-Value \\
\hline Voltage & 29.75 & 2 & 14.87 & 59.71 & $3.68 \times 10^{-12}$ \\
\hline Mass Flow & 28.79 & 2 & 14.40 & 57.8 & $5.76 \times 10^{-12}$ \\
\hline Shim Level & 42.47 & 4 & 10.62 & 42.62 & $3.51 \times 10^{-13}$ \\
\hline Error & 8.97 & 36 & 0.25 & & \\
\hline Total & 109.97 & 44 & & & \\
\hline
\end{tabular}




\section{Chapter 5: Ion Energy Characterization}

Measurement of the ion energy in a Hall thruster plume can provide direct insight into the acceleration processes in the thruster. Any change in the height of the effective acceleration voltage will be reflected in the ion energies in the plume. Ion energy (and the corresponding ion velocity) are primary factors in both the thrust and specific impulse of the thruster. Determination of the ion energy will therefore aid greatly in determining the source of the performance changes seen in the thruster. Ion energy should behave similarly to thruster specific impulse; as both are effectively measuring the speed with which ions leave the thruster, both properties should show similar behavior.

\subsection{RPA}

A Retarding Potential Analyzer (RPA) is a gridded probe which is used to determine the directed ion energies present in a plasma ${ }^{27}$ and has been used to good effect in Hall thruster plume studies. ${ }^{28,29}$ An RPA provides a potential hill, which ions must climb in order to be detected. If this hill is much smaller than the accelerating potential hill in the thruster, all of the beam ions will pass over the hill and be collected. If the hill is larger than the potential hill in the thruster, no ions will be collected as they cannot 'roll up' the large potential hill in the probe. In this way, an RPA functions as a high-pass filter, allowing only those ions of sufficient energy to roll up the potential hill in the probe to be detected. Probes of this type usually filter out the plasma electrons so as to measure only the ions.

Determination of the ion distribution function from RPA data is the primary motivation for using the probe. The ions and electrons in a plasma have a distribution function, which determines what the probability is that an ion or electron will be found with a given energy or velocity. Determination of the distribution function can be begun by examining the current incident on a collecting surface:

$$
I=q e \Phi A
$$

The flux of ions on the surface is calculated from the ion density and average velocity:

$$
\Phi=n\langle v\rangle
$$


In this case, it is inappropriate to use the true average velocity. Rather, the average velocity perpendicular to the probe surface can be used. Additionally, as the ions can be assumed to have a bulk speed towards the collector, this becomes the average speed in the direction normal to the probe surface. The average velocity of a population of particles in a given direction, independent of the nature of the distribution function can be given by: ${ }^{30}$

$$
<v>=\int_{-\infty}^{\infty} v f(v) d v
$$

The lower bound of the integration can be replaced with a minimum velocity $\mathrm{v}_{\min }$, which is the minimum velocity of ions which can enter and be detected by the probe. $V_{\min }$ is a positive value for the purposes of this integration, as ions traveling in the direction opposite the probe (negative velocity) cannot enter and be detected. Substituting the ion density and average velocity for flux into the equation for current results in:

$$
I=q e n A \int_{v_{\min }}^{\infty} v f(v) d v
$$

The relation for current as a function of speed can be converted to a function of probe voltage through a change of variables. This is accomplished by replacing speed with the voltage required to prevent the ions from passing over the potential hill in the probe and being detected. Ion speed is related to kinetic energy through the equation:

$$
K=1 / 2 M_{x e} v^{2}
$$

As the potential required to accelerate an ion to kinetic energy $K$ (and hence, decelerate an ion of kinetic energy $\mathrm{K}$ to zero) is trivially found by multiplying the kinetic energy by the charge of the ion qe, the potential required to repel an ion with speed $v$ is thus:

$$
V=\frac{M_{x e} v^{2}}{2 q e} .
$$

Rearranging the equation for potential as a function of speed leads to:

$$
v=\sqrt{\frac{2 q e V}{M_{X e}}} .
$$


This allows substitution for the velocity in Eqn. 5-4. Replacing the $d v$-term simply required the derivative of this substitution:

$$
d v=\frac{1}{2} \sqrt{\frac{2 q e}{M_{X e} V}} d V .
$$

Substituting for $v$ and $d v$, replacing $f(v)$ with $f(V)$, thus eliminating the dependence of the distribution function on the velocity, yields the equation:

$$
I=A q e n \int_{V_{\min }}^{\infty} \sqrt{\frac{2 q e V}{M_{X e}}} \frac{1}{2} \sqrt{\frac{2 q e}{M_{X e} V}} f(V) d V,
$$

which can be simplified to:

$$
I=A e^{2} \frac{n q^{2}}{M_{X e}} \int_{V \min }^{\infty} f(V) d V
$$

The $\mathrm{V}_{\min }$ term can be defined here as the repelling voltage in the probe. Ions which were accelerated through a potential lower than $V_{\min }$ will be repelled by the probe and not detected, while ions accelerated through potentials equal to or greater than $\mathrm{V}_{\min }$ will be seen by the probe. Differentiating the current with respect to probe voltage, assuming that the value of the distribution function at infinity is zero, yields the equation:

$$
-\frac{d I}{d V}=A e^{2} \frac{n q^{2}}{M_{X e}} f(V) .
$$

This result shows that the derivative of the current seen by the RPA is directly proportional to the distribution function. The exact values of the collector area, ion density, and ion charge states are not known, so they can be combined into a single normalization constant $\mathrm{N}$, yielding:

$$
\frac{d I}{d V}=-N f(V)
$$

\subsection{RPA Design}

The most basic RPA can be constructed with a single grid and a collector, as shown in Figure 5-1. Ions approaching the grid roll up a potential hill equal to the voltage on the grid, and thus ions with 
insufficient energy to pass the potential hill are not detected. As the grid voltage is increased, ions of higher energies are repelled, with the result that when the grid voltage approaches and then begins to exceed the potential hill the ions rolled down on acceleration by the thruster, the probe will reject a significant fraction of the ions. Eventually the grid reaches a potential high enough that all ions are repelled, thus causing no current on the detector.

In practice, an RPA is a more complex probe. A single grid, biased to repel positive ions, is unable to prevent plasma electrons from reaching the collector. Electrons impacting the collector will register as the negative of an ion reaching the collector, thus canceling out an equal amount of ion current and severely impacting the current measurement. Electrons can also be emitted from the collector through secondary electron emission. If secondary electrons were allowed to escape, they would appear in the data as the collection of two ions, as the positive ion impacting the collector and the negative electron leaving the collector are indistinguishable to a current measurement. To avoid complications from plasma and secondary electrons, two grids are commonly added, one upstream and one downstream of the ion repeller grid. Both of these grids are biased negative, with the result that the upstream grid repels plasma electrons, preventing them from entering the probe. The negative bias on the downstream grid deflects secondary electrons emitted from the collector back towards it, thus negating any effect they may have on the current measurement. Due to their function, these grids are called the electron repeller and secondary electron

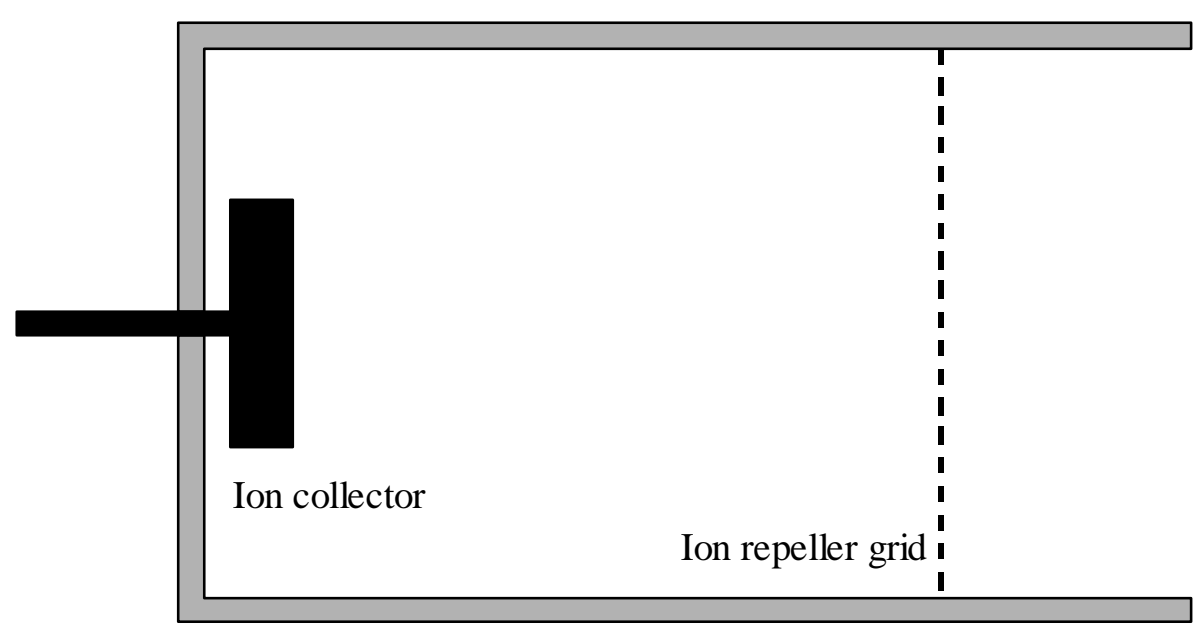

Figure 5-1: Single-grid RPA 
repeller grids, respectively.

A fourth grid is commonly added in many RPAs, as shown in Figure 5-2. This grid is called the entry grid, and is placed at the entrance of the probe, upstream of the electron repeller grid. This grid is biased at either the ambient plasma potential or isolated from ground and allowed to float. In doing so, the entry grid limits the perturbation to the surrounding plasma, by screening out the applied voltages on the other grids.

The RPA utilized in the experiments reported here is of the four-grid design. All conductive components of the RPA are constructed from stainless steel. The grids are fabricated from stainless steel mesh with a $0.139-\mathrm{mm}$ average grid gap, and $30 \%$ open-area fraction. The electrical connection to the grids are made via stainless-steel wire, run through the casing of the probe and welded to each grid. Alumina-silicate ceramic spacers separate the grids. The entry orifice of the probe is $12.7 \mathrm{~mm}$ in diameter. The front face of the probe is fastened with screws to the outer casing, and serves to press

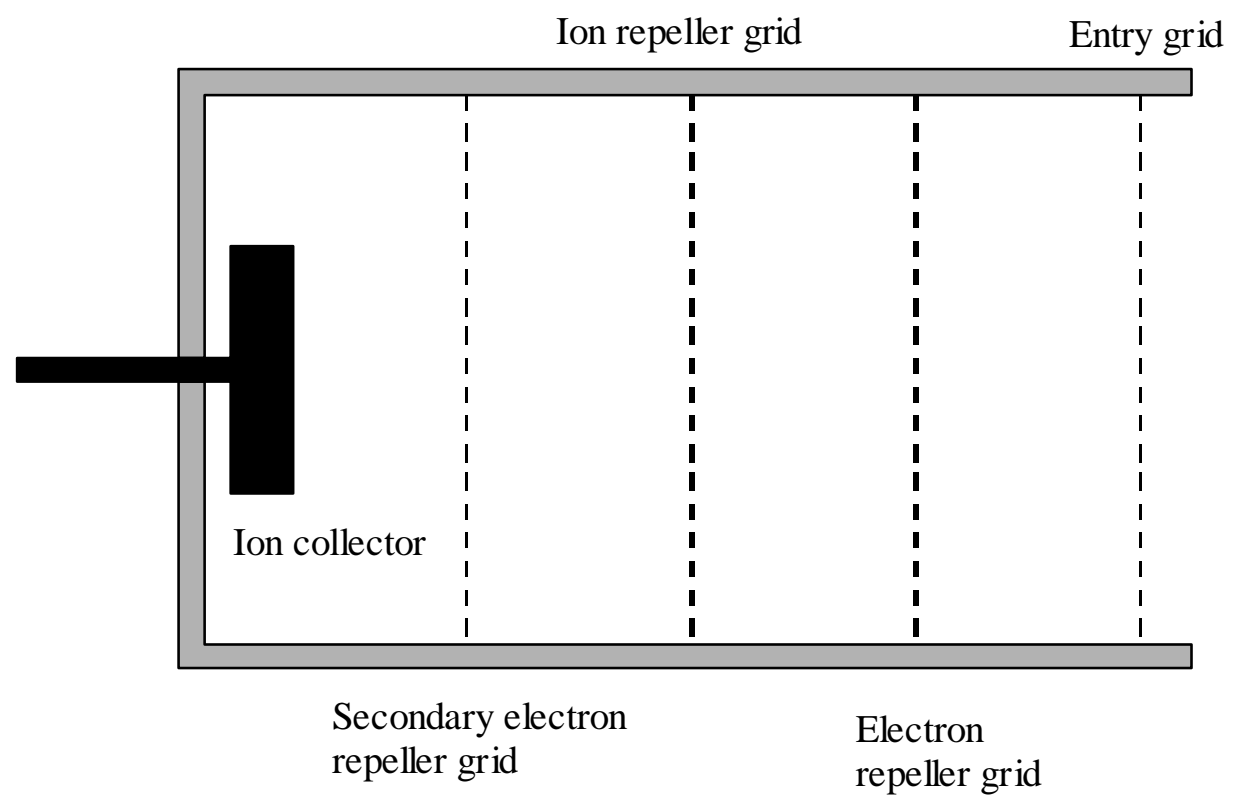

Figure 5-2: Four-grid RPA 


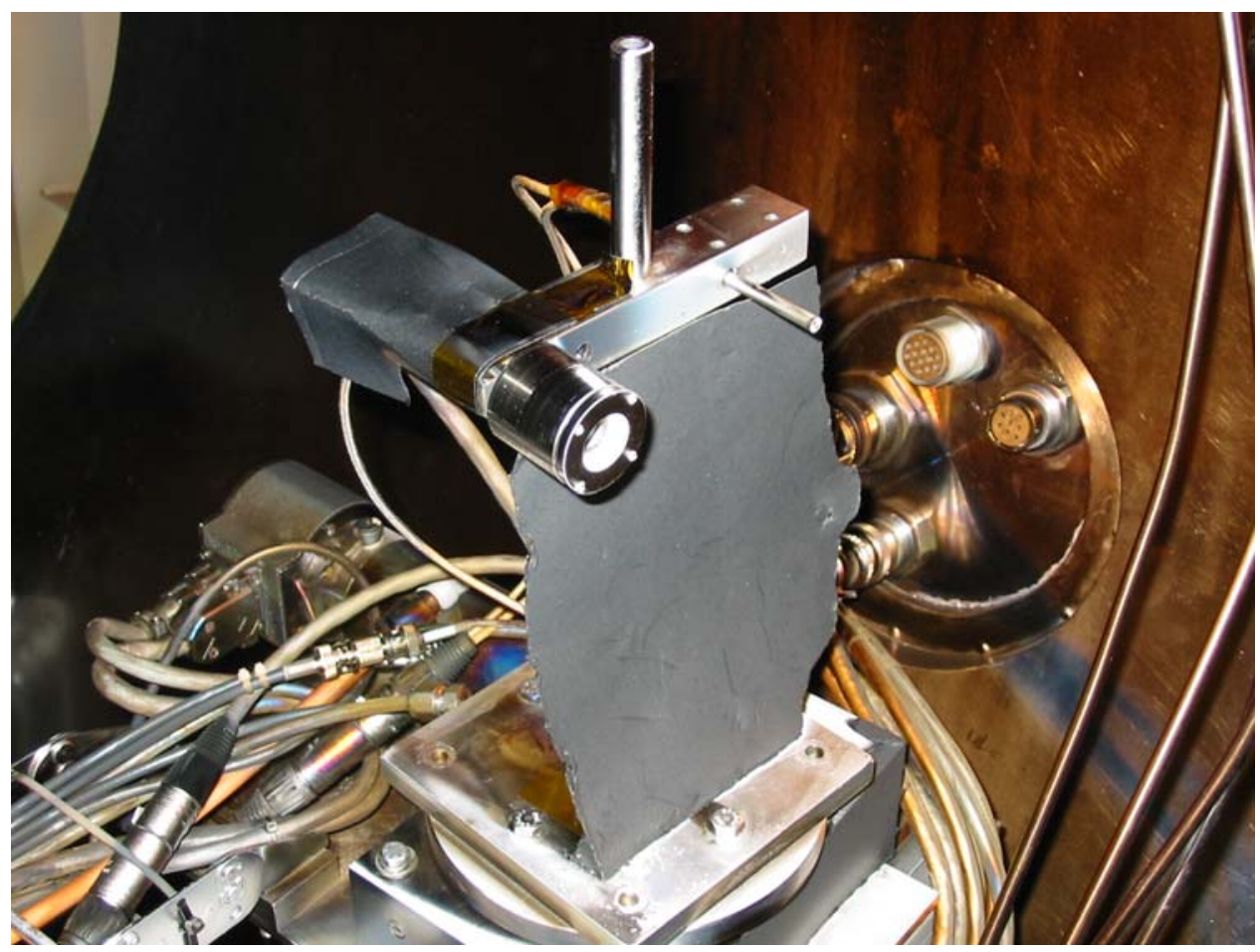

Figure 5-3: Photograph of an RPA in the vacuum facility

the grids and spacers together, preventing any movement during testing. The entry grid of the probe was in contact with the front face, and thus was floating at all times during testing.

The ion repeller grid of the RPA was swept from zero to as much as $600 \mathrm{~V}$ above ground. The maximum voltage varied according to thruster operating point; higher thruster voltages required higher ion repeller voltages to filter out all of the beam ions. Sweeping was accomplished through use of a Keithley 2410 in voltage sweep mode. Electron and secondary electron repeller grids were set at $10 \mathrm{~V}$ below ground and remained at the same potential through the entire testing sequence. The value of $10 \mathrm{~V}$ was chosen so as to be significantly higher than the voltage necessary to reject the plasma electrons on the electron repeller grid, which usually required a potential of only $2 \mathrm{~V}$ below ground. The current to the collector was amplified and converted to a voltage signal by an analog current amplifier, with a gain setting of either $10^{3}$ or $10^{4}$ volts per amp, dependent on the thruster operating point. An oscilloscope then read this voltage. The ion repeller voltage controlled the oscilloscope triggering, so that the current detected by the probe as a function of voltage could be easily extracted. A typical RPA probe trace with its associated derivative is plotted in Figure 5-4. 


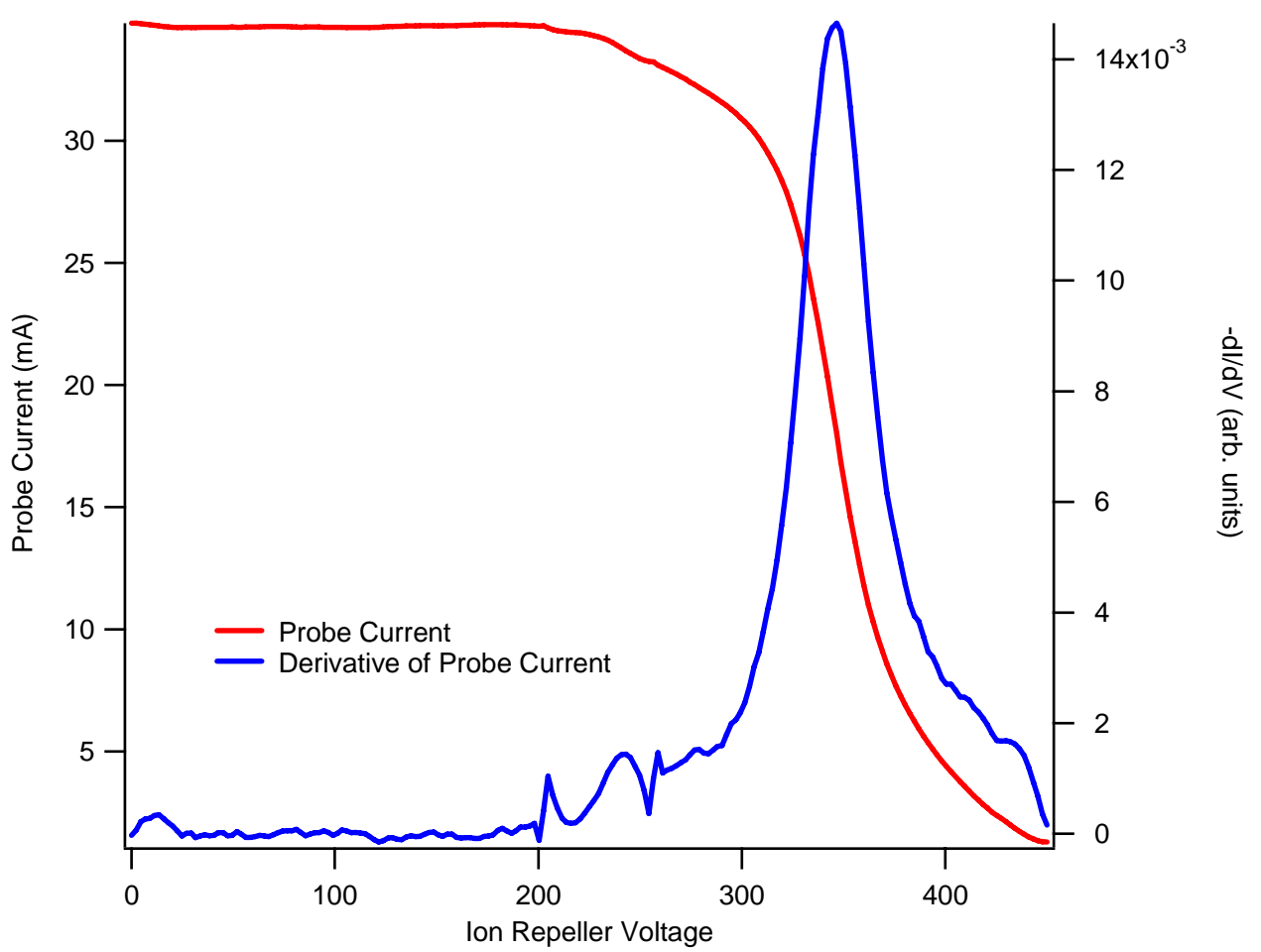

Figure 5-4: Typical RPA I-V sweep and associated derivative of current with respect to voltage, with $350 \mathrm{~V}$ on the anode, $5 \mathrm{mg} / \mathrm{s}$ of propellant flow, and all discharge heating on the shims.

A complication to the determination of ion energy from the RPA is that the ion repeller voltage is measured with respect to ground. There is an ambient potential in the plasma (plasma potential), through which ions will be accelerated or decelerated relative to ground. For a plasma potential ten volts above ground, ions will be accelerated through a ten-volt potential when passing through a grounded grid, thus ten volts must be subtracted from the ion repeller voltage in post-processing to account for the acceleration due to the plasma potential. Measurement of the plasma potential was accomplished through use of an emissive probe.

\subsection{Emissive Probe}

An emissive probe consists of a hot filament, heated such that it will emit electrons. ${ }^{31}$ At potentials below the plasma potential, the plasma electrons will be deflected away from the probe due to its negative potential relative to the plasma repelling the electrons. At potentials above the plasma potential, the probe will attract and collect plasma electrons. The purpose of the emission is that when the probe is below plasma potential, it will emit electrons, which are immediately repelled from the probe by the 
difference between it and plasma potential. For potentials above plasma potential, any emitted electrons are attracted to the filament by its high potential relative to the plasma, and thus provide no net current. This change in behavior between potentials above and blow plasma potential causes a large change in incident current on the probe due to the plasma. At high potentials, the probe current reaches a saturation point where emitted electrons are being recollected, and any plasma electrons capable of penetrating the Debye sheath around the probe are being collected. Only electrons which move into the sheath through thermal or other non-probe-induced motion can be collected, so the current will increase very slowly with voltage. This increase is due to the sheath increasing in size slightly as the probe reaches higher voltages. At potentials below plasma potential, the rate of change of current as a function of voltage is greatly different. The electron current decreases rapidly as the voltage is reduced from plasma potential. This is due to the fact that increasing potentials below the plasma potential give the electrons on the surface of the filament a larger and larger potential hill to fall down when they escape, thus making escape from the surface easier.

The plasma potential can then be found by examining the intersection between the shallow electron saturation regime and the steep emissive regime. The specific method used for this was to find a linear fit to the I-V characteristic in both the emission and electron saturation regimes, and find the point where the two fits intersected. ${ }^{32}$ The voltage at the intersection was the plasma potential. A typical emissive probe trace with the associated linear fits is plotted in Figure 5-5.

The emissive probes utilized consisted of 0.127 -mm-diameter thoriated tungsten filaments (2\% thorium content). The purpose of the thorium is to reduce the work function of the material, increasing the emission current and allowing the probe to be operated at lower temperatures. The probe filaments were approximately $5 \mathrm{~mm}$ long. Current was passed along the filaments to heat them to incandescence and allow them to emit electrons. The current necessary for onset of electron emission was approximately 2.6 amps, and the hot probes exhibited a resistance of approximately 2 ohms, varying slightly from probe to probe. Due to the fragile nature of the filaments, the probes required filament replacement after each thruster test as the filaments either broke during operation or became brittle. 


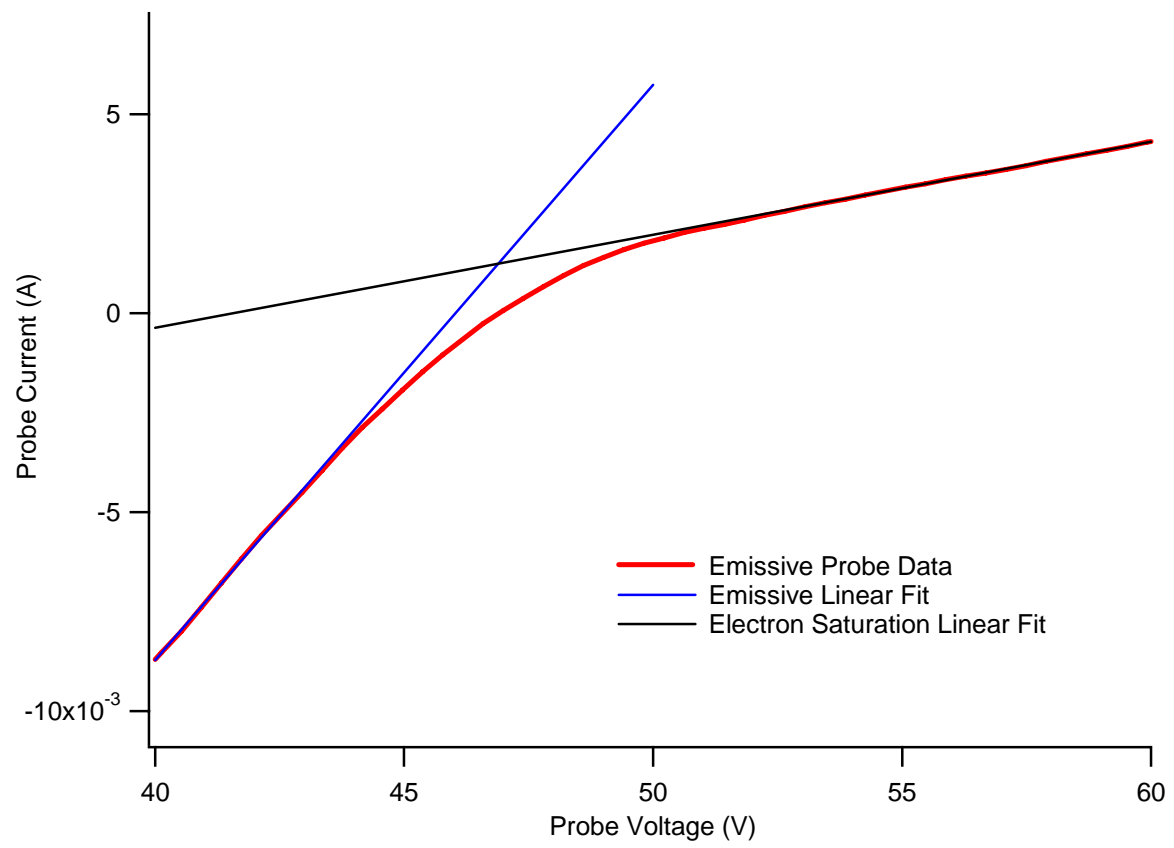

Figure 5-5: Typical emissive probe trace

\subsection{Determination of Plasma Potential}

Plasma potential was calculated from the emissive probe I-V characteristics, and has been plotted in Figure 5-6. The probe was located at the same point in the thruster plume in which RPA data were acquired, in this case on the thruster axis $500 \mathrm{~mm}$ downstream of the exit plane, thus eliminating error in the RPA measurements due to the plasma potential being different from that measured. The first conclusion that can be made based on these data is that any the changes in plasma potential as the experimental factors are modified are small. At nearly all combinations of anode voltage and propellant mass flow, the plasma potential was highest at high shim voltages (all discharge heating on the shims), and lowest at low shim voltages. The only exception to this was the 356-set of operating conditions, where the plasma potentials at the high and low shim voltage settings were roughly equal. The increase in plasma potential as discharge electron heating was diverted from the main anode to the shims was not linear; in several cases most of the increase in plasma potential came about between $25 \%$ and $75 \%$ of the discharge heating on the shims. One significant outlier on the plasma potential data is the 304-set of operating conditions. While the 304-0, 304-75, and 304-100 operating conditions have plasma potentials near the 305- and 306-sets of operating conditions, the plasma potential in the 304-25 and especially in the 304-50 conditions are smaller than the plasma 


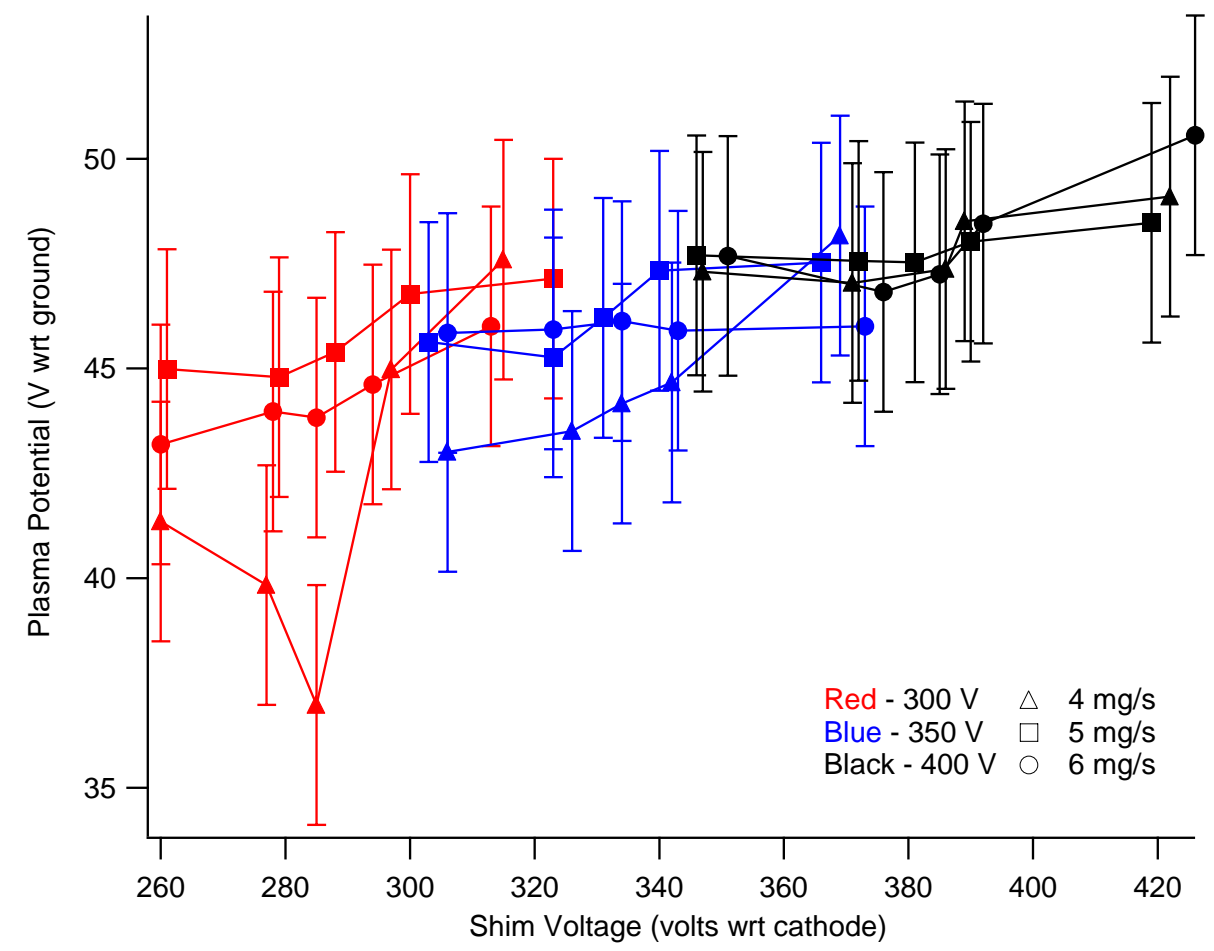

Figure 5-6: Plasma Potential relative to ground as a function of all three experimental factors, measured on the axis of the thruster, $500 \mathrm{~mm}$ from the exit plane.

potentials measured at all other operating points. These will contribute significantly to the experimental error estimate.

Analysis of the plasma potential data by the analysis of variance method shows that all three experimental factors had significant effects on the plasma potential, as shown in Table 5-1. While the Pvalues were much larger than those calculated for many of the other measurements taken such as thrust, specific impulse, and beam divergence, they are still significantly smaller than $1 \%$, and thus the null hypothesis can be dismissed as incorrect. Using the MSE calculated from the analysis of variance, the $95 \%$ confidence interval on the data was calculated to have a width of $2.9 \mathrm{~V}$. This error is quite large; $5.7-7.7 \%$ of the measured plasma potential. Furthermore, the error is several times larger than the change seen in plasma potential as many of the experimental factors were changed. While this large error does not change the conclusion that the plasma potential was significantly affected by all three experimental factors, it complicates the use of plasma potential in adjusting the ion repeller voltages used by the RPA. 
Table 5-1: ANOVA table of Plasma Potential

\begin{tabular}{|c|c|c|c|c|c|}
\hline $\begin{array}{c}\text { Experimental } \\
\text { Factor }\end{array}$ & Sum of Squares & $\begin{array}{c}\text { Degrees of } \\
\text { Freedom }\end{array}$ & Mean Squared & $\mathrm{F}_{0}$ & P-Value \\
\hline Anode Voltage & 113.0 & 2 & 56.5 & 28.4 & $3.95 \times 10^{-8}$ \\
\hline Mass Flow & 25.1 & 2 & 12.5 & 6.3 & .0045 \\
\hline Shim Level & 58.0 & 4 & 14.5 & 7.3 & .00021 \\
\hline Error & 71.6 & 36 & 2.0 & & \\
\hline Total & 267.7 & 44 & & & \\
\hline
\end{tabular}

\subsection{Measurement of Ion Energy}

Ion energy was measured using a retarding potential analyzer. The plasma- and secondaryelectron repellers in the probe were biased $10 \mathrm{~V}$ below ground to ensure no electrons from either the plasma or from secondary electron emission could affect the measurements. The entrance grid was physically connected to the probe shell and allowed to float, thus screening out the high voltages inside the probe, and minimizing the effect on the plasma. The ion repeller voltage was swept between 0 and up to $600 \mathrm{~V}$ at roughly $0.5 \mathrm{~Hz}$. The data were averaged via an oscilloscope up to eight times before output to a computer, to minimize the effect of noise on the measurements.

After normalization and taking the derivative of the probe current the average ion energy was calculated. Both the most probable energy and the average energy are of interest, however, as they each can provide insight into the ion acceleration process in the thruster. A change in the most probable ion energy indicates a change in the acceleration potential at the point where the majority of ions are accelerated, while changes in the average ion energy indicate a net increase or decrease in ion acceleration potential. These are not necessarily the same; an acceleration potential structure could be produced which will accelerate most of the ions to a given energy, but produce a significant population of ions at either a higher or lower velocity than the most probable. These populations of fast or slow ions would affect the average, despite the fact that the most probable ion velocity may be the same as in another operating condition.

The derivatives of probe current as a function of ion repeller voltage are plotted in Figures 5-7 - 59. The currents were normalized to unity before differentiating, thus the ion energy distribution functions calculated are area normalized and show the trend in the ion energy distribution. Aside from the expected increase in ion energy with anode voltage (and corresponding increase in shim voltage), the ion energy 
showed a significant change between the condition with all current on the shims and the other levels of shim current. In all of the cases displayed, the peak of the ion energy distribution was 20-30 eV/q higher when all discharge current was on the shims than any other amount of shim current. This indicates a strong dependence on shim voltage, but only at operating conditions where the shim voltage is higher than the main anode voltage. In each of these cases, the shim voltage was approximately equal to the main anode voltage with $75 \%$ of the discharge heating on the shims, and the shim voltage was always lower than the anode voltage when the shims were accepting less than $75 \%$ of the discharge current. These data suggest that the ion acceleration is governed by the highest potential present in the thruster: Shim voltage governs at high shim voltages, and anode voltage governs at low shim voltages. Another conclusion that can be drawn is that the ion distribution function is more spread out when all of the discharge current was on the shims. The short peak of the normalized distribution with all discharge heating on the shims indicates that the ion acceleration process is more spread out at that operating condition than when the shims are accepting less of the discharge electron heating. Thus while the most probable ion energy is higher when all discharge heating is on the shims, the thruster is accelerating ions over a much wider range of energies. 

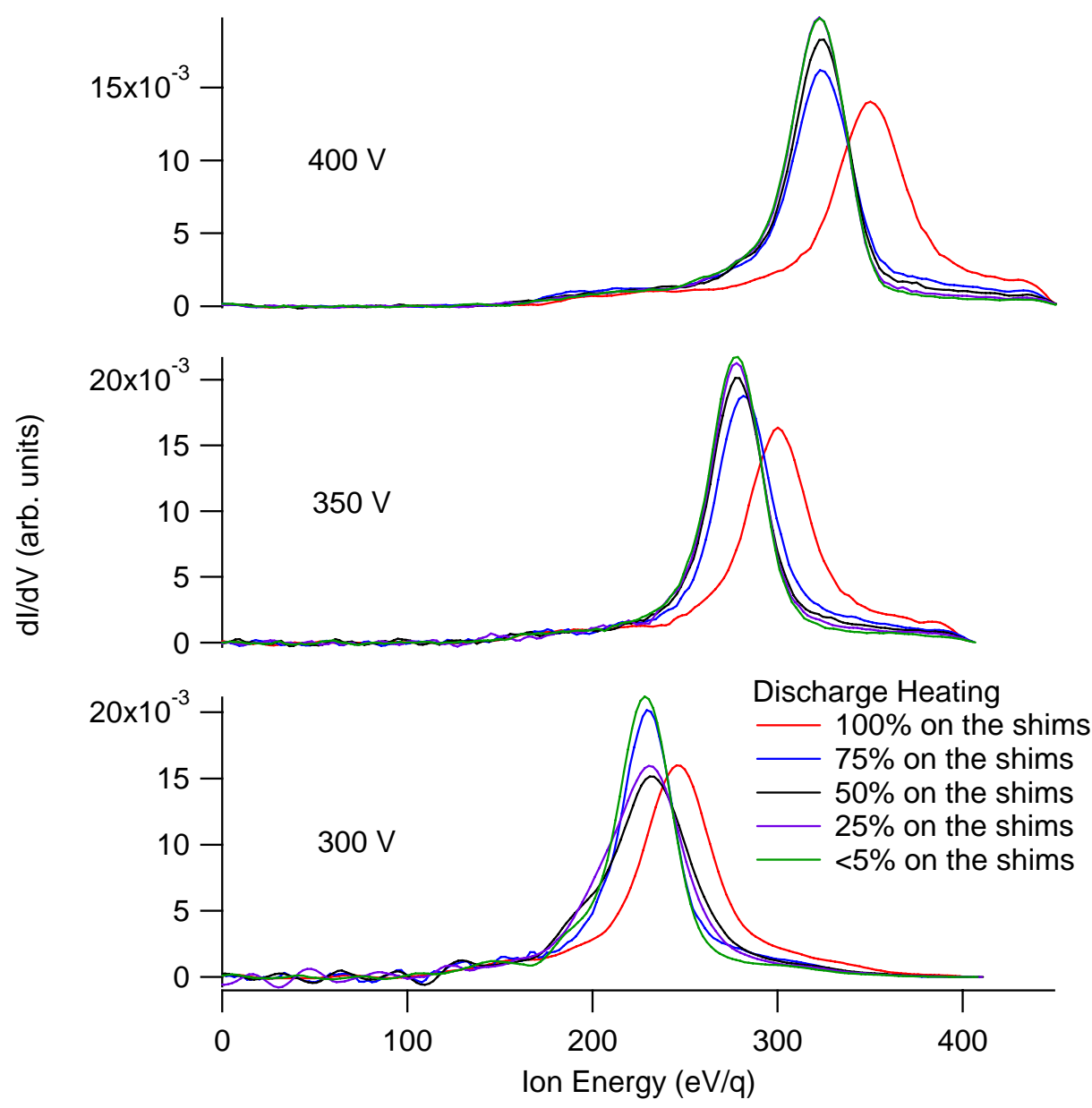

Figure 5-7: Derivatives of RPA current with at $4 \mathrm{mg} / \mathrm{s}$ of xenon, at all combinations of anode voltages and shim levels 


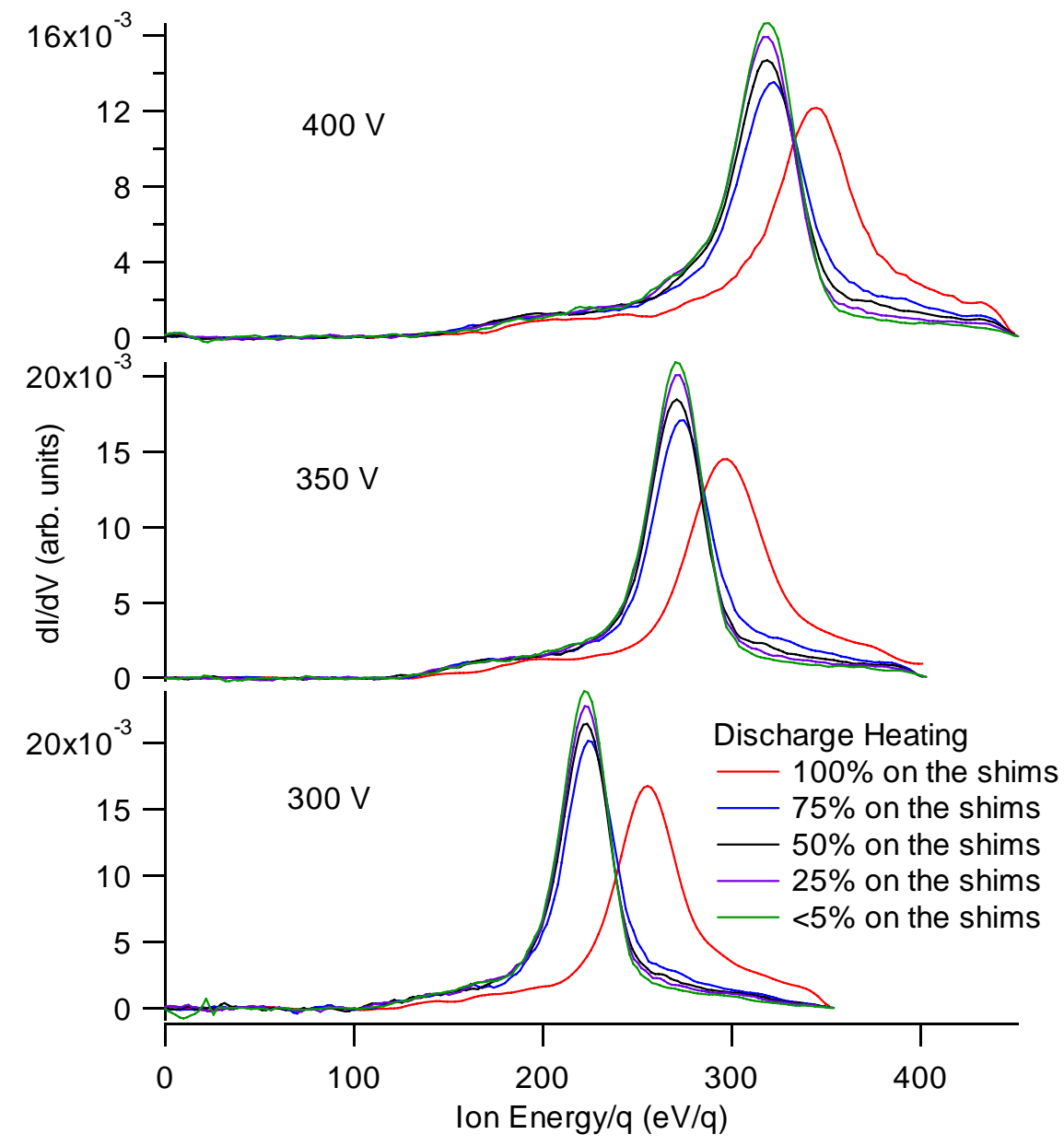

Figure 5-8: Derivatives of RPA current with at $5 \mathrm{mg} / \mathrm{s}$ of xenon, at all combinations of anode voltages and shim levels 

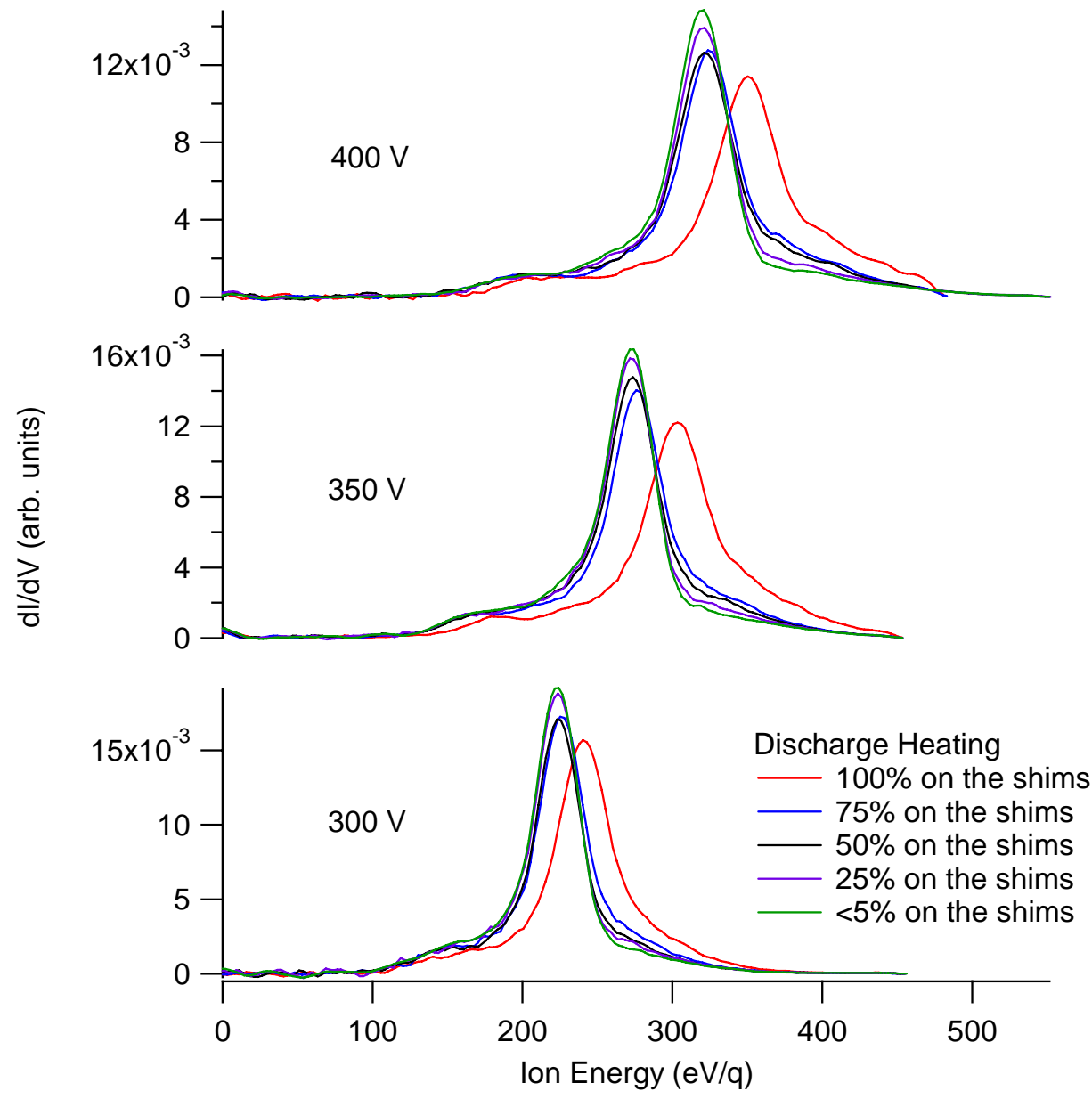

Figure 5-9: Derivatives of RPA current with at $6 \mathrm{mg} / \mathrm{s}$ of xenon, at all combinations of anode voltages and shim levels

The most probable ion energy, as plotted for all operating conditions in Figure 5-10, verifies the strong dependence seen in Figured 5-7 - 5-9 on anode and shim voltages. The ion energies show an approximately $50 \mathrm{eV}$ increase with each $50 \mathrm{~V}$ increase in anode voltage. Thus the effect of the anode voltage on the ion energy is roughly linear, with increases in anode voltage being mirrored in the most probable ion energy. The changes in ion energy as anode voltage was adjusted are expected, as alterations to the anode potential directly adjust the electric field through which the ions are accelerated. Very little change is seen in ion energy as a function of mass flow. The change in ion energy as a function of shim voltage verifies the dependence on the highest voltage in the thruster seen in Figures 5-7 - 5-9. For shim voltages above the main anode voltage, the ion energy appears to increase with shim voltage. At shim voltages at or below anode voltage, however, the ion energy becomes somewhat independent of the shim voltage; further reductions of shim potential below anode potential do not affect the ion energy. These 
results indicate that the highest voltage present in the thruster determines the most probable ion acceleration potential, whether that is anode or shim voltage. While it cannot determine the exact location at which the acceleration takes place, measurement of the most probable ion energy has revealed that the ion acceleration process is governed by the shim voltage in a slightly different way than the specific impulse; while specific impulse decreased with shim voltage, the ion energy appears to hit a minimum energy, dictated by the anode voltage, and remains roughly at that voltage for all operating points where the shim voltage was below anode voltage.

Application of the analysis of variance to the most probable ion energy, calculated from the ion energy distribution functions, verifies the expected dependence of ion energy on the anode and shim voltages in the thruster, as shown in Table 5-2. All three of the experimental factors have a significant effect on the most probable ion energy, however the contribution from anode voltage and shim level are the majority components of the change, with mass flow having a much less significant effect. The 95\% Confidence interval, has a width of $7 \mathrm{eV} / \mathrm{q}$. This is much smaller as a fraction of the measurement than many of the other errors calculated; the error is between 2 and $3.2 \%$ of the

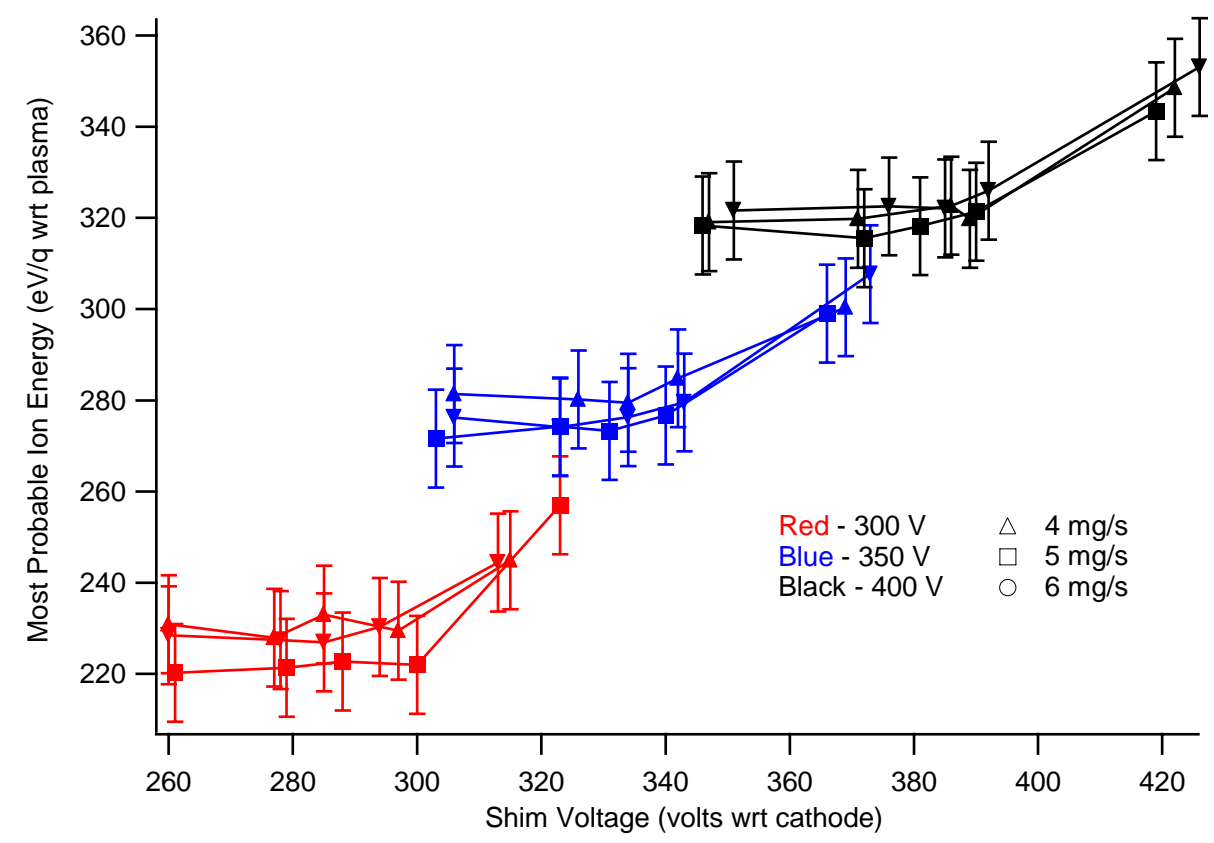

Figure 5-10: Most Probable Ion Energy as a function of all three experimental factors. Higher shim voltage indicates a higher proportion of discharge current on the shims. 
Table 5-2: ANOVA table of most probable ion energy

\begin{tabular}{|c|c|c|c|c|c|}
\hline $\begin{array}{c}\text { Experimental } \\
\text { Factor }\end{array}$ & Sum of Squares & $\begin{array}{c}\text { Degrees of } \\
\text { Freedom }\end{array}$ & Mean Squared & $\mathrm{F}_{0}$ & P-Value \\
\hline Anode Voltage & 67773.3 & 2 & 33886.6 & 2817.2 & $2.81 \times 10^{-40}$ \\
\hline Mass Flow & 186.5 & 2 & 93.2 & 7.8 & $1.59 \times 10^{-3}$ \\
\hline Shim Level & 4525.0 & 4 & 1131.3 & 94.0 & $1.52 \times 10^{-18}$ \\
\hline Error & 433.0 & 36 & 12.0 & & \\
\hline Total & 72917.8 & 44 & & & \\
\hline
\end{tabular}

measurement. Unlike the trends in thrust and specific impulse, the changes in ion energy with the most significant factors (anode voltage and shim level) are larger than the confidence interval; thus the anode voltage and level of discharge electron heating on the shims are both primary contributing factors to the most probable ion energy in the exhaust plume of the hall thruster.

The distributions plotted in Figures 5-7 - 5-9 show ion energy distributions that are asymmetrical, with both a high and low energy 'tail.' The average ion energy is then calculated by integrating the product of the energy distribution function and energy:

$$
\bar{E}=\int E f(E) d E
$$

. These data are plotted in Figure 5-11, and show a much more complex behavior than that shown in Figure 5-10. The ion energy data show that average ion energy decreased with shim voltage, and did not reach a minimum ion energy when the shim voltage was lower than the anode voltage. This means that, while the most probable ion energies were not changing significantly between the $50 \%, 25 \%$, and $<5 \%$ of discharge current on the shims operating points, the average energies were. Thus while most of the ions saw the same acceleration potential at these operating points, the proportion of ions which saw less acceleration became larger, thus leading to the lower average energy. This change could also be due to a reduction in high-energy ions. Both of these conclusions could be true. In Figure 5-8, the low-energy tails of the ion energy distributions appeared to become larger and more pronounced, while the high-energy tails became smaller. This double effect caused the average ion energy to decrease. This result agrees well with the observed reduction in thrust and specific impulse with shim voltage. Both the thrust and specific impulse were reduced with shim voltage, and the reduction in ion energy seen in Figure 5-11 verifies that the reduction in thrust is due at least in part to the energy the ions gain when accelerated out of the thruster. 


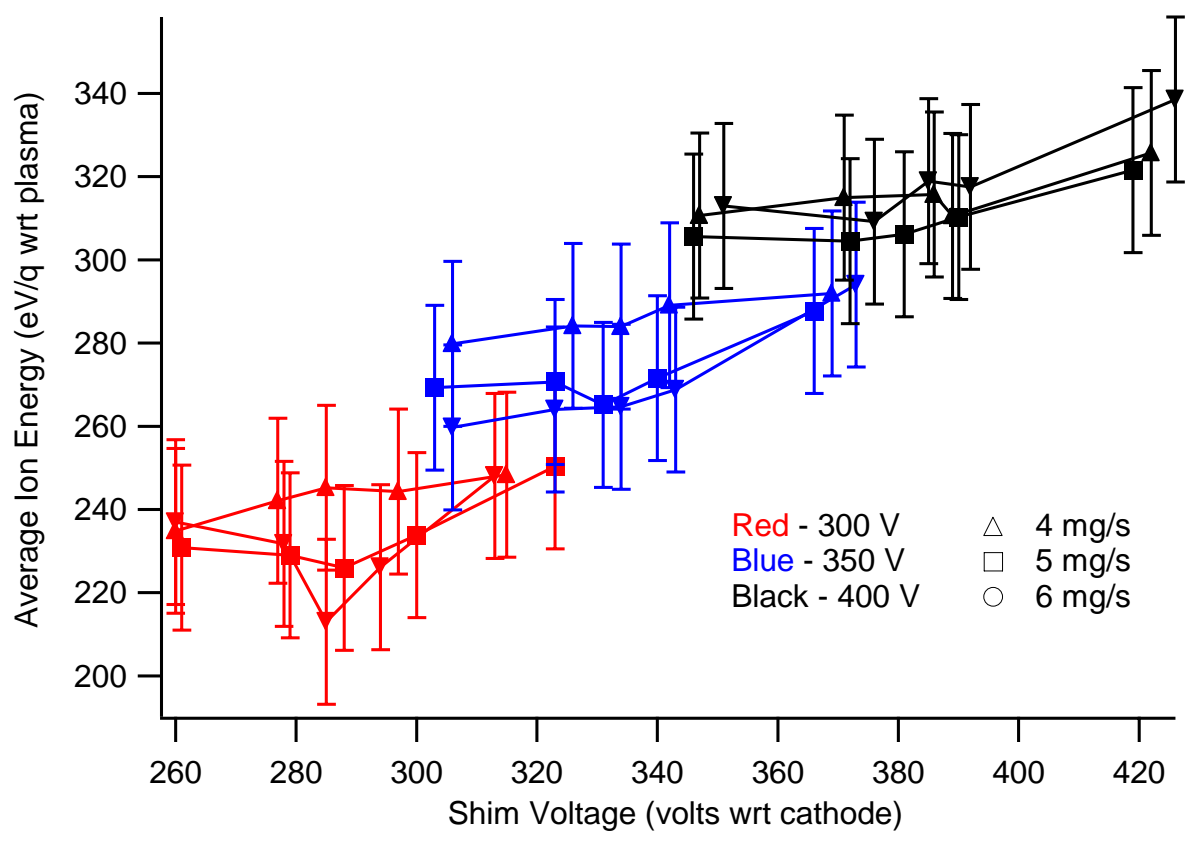

Figure 5-11: Average ion energy as a function of all three experimental factors. Higher shim voltage for a given combination of anode voltage and mass flow indicates higher discharge heating on the shims

Application of the analysis of variance to the average ion energy provides similar conclusions to those made with the most probable ion energy, as shown in Table 5-3. All three of the experimental factors are found to be significant, with the anode voltage being the largest contributor to average ion energy. Shim level (and thus shim voltage) has the second-largest effect on average energy, and mass flow has the smallest effect. These agree well with the analysis of the data as presented in Figure 5-11. The expected error, however, is much larger than that for the most probable ion energy. The 95\% confidence interval on the average ion energy has a width of $13 \mathrm{eV} / \mathrm{q}$, nearly twice the uncertainty in the most probable ion energy, and between 3.8 and $6.1 \%$ of the calculated average energy. This is due primarily to the much larger error inherent in the calculation of average energy; most probable energy is found simply by determining the ion energy at the peak of the distribution. The error in average energy will be larger because it is the product of the ion energy and the derivative of the normalized probe current, both of which have errors on the order of the most probable energy. In the average ion energy error approximation, unlike the most probable ion energy approximation, the uncertainty in the data is larger than the change due to not only mass flow, but also shim voltage. The large uncertainty does not prevent the factors from having a significant affect on the average ion energy, however it complicates determination of the nature of 
the effect. Further analysis of the average ion energy requires knowledge of the ion multiple charge

fraction. Addition of the effect of multiply charged ions will allow determination of the ion energy from the ion energy per unit charge, which will provide a much more accurate picture of the kinetic energy of ions in the plume, and thus allow comparison with the performance measurements.

Table 5-3: ANOVA table of average ion energy per unit charge

\begin{tabular}{|c|c|c|c|c|c|}
\hline $\begin{array}{c}\text { Experimental } \\
\text { Factor }\end{array}$ & Sum of Squares & $\begin{array}{c}\text { Degrees of } \\
\text { Freedom }\end{array}$ & Mean Squared & $\mathrm{F}_{0}$ & P-value \\
\hline Anode Voltage & 46627.2 & 2 & 23313.6 & 564.8 & $6.5 \times 10^{-28}$ \\
\hline Mass Flow & 743.2 & 2 & 371.6 & 9.0 & $6.8 \times 10^{-4}$ \\
\hline Shim Level & 2239.7 & 4 & 559.9 & 13.6 & $7.7 \times 10^{-7}$ \\
\hline Error & 1485.9 & 36 & 41.3 & & \\
\hline Total & 51096.1 & 44 & & & \\
\hline
\end{tabular}




\section{Chapter 6: Ion Multiple Charge Characterization}

The number of multiply charged ions present in a Hall thruster discharge has a direct effect on performance. The amount of energy gained by ions as they roll down the potential hill produced in the thruster is dependant on their charge, due to the electrostatic nature of the acceleration. The height of the potential hill is multiplied by the charge of an ion when determining the kinetic energy gained, thus multiply charged ions will travel much faster than those with just one unit of charge. This increase in ion energy is manifested in increased thrust and specific impulse, but a net decrease in efficiency. Recalling the equation:

$$
\eta=\frac{F^{2}}{2 \dot{m} P} .
$$

It is difficult to determine if the thruster efficiency is affected by the presence of multiply charged ions purely from the acceleration kinetics. Under the assumption that all ions are accelerated through the same potential, independent of charge state, the argument can be made that production of multiply charged ions does not affect thruster efficiency. Two factors contribute to this difficulty. First is that the thrust force from a flow of ions is proportional to the square root of the ion energy. Energy increases linearly with charge, so force will increase as the square root of the ion charge. The second factor is the power required to accelerate a multiply charged ion. This increases linearly with charge, as power is calculated as a voltage multiplied by a current. Voltage in the thruster is invariant, but current is linearly affected by charge. So the thrust force will increase as the square root of charge, while the power will increase with charge. In Eqn. 3-6, the numerator and denominator both will increase linearly with charge, as thrust force is squared. Thus multiply charged ions will not affect the thruster efficiency, assuming equal accelerating potentials for all species. The assumption that accelerating potential is equal for all charge species cannot be made in a real thruster, however, and without intimate knowledge of the accelerating potential inside the thruster and the locations of ion production a more accurate model cannot be used. Thus pure acceleration kinetics cannot do a good job of predicting whether efficiency will change as multiply charged ions are produced. 
The area where multiply charged ions will always affect efficiency is in ion production cost. It costs $12.1 \mathrm{eV}$ to ionize one xenon atom. To doubly ionize an atom requires $21.2 \mathrm{eV}$ above that required for production of a singly charged ion (33.3 eV total to doubly ionize a neutral xenon atom), thus creation of one doubly charged xenon ion is more expensive energetically than the production of two singly charged xenon ions. $32.1 \mathrm{eV}$ are required to create a triply charged xenon ion from a double; $65.4 \mathrm{eV}$ total to obtain triply charged xenon from the neutral atom. Thus any significant increase in the multiply charged ion fraction will significantly increase the power required for ionization, lowering total thruster efficiency.

The increased velocity of multiply charged ions also causes a reduction in thruster lifetime. As seen in Appendix A, the erosion rate of a surface under ion bombardment is directly related to the ion energy. Multiply charged ions, then, with their much higher exhaust velocities than singly charged ions, will increase the rate of thruster erosion and reduce lifetime.

\subsection{ExB Probe}

An ExB probe uses perpendicular electric and magnetic fields to filter ions by velocity, as shown in Figure 6-1. ExB probes have been successfully used for characterization of Hall thruster plasmas by several researchers. ${ }^{23,33}$ This is an important distinction from the filtering in an RPA. Due to the electrostatic nature of the RPA, it cannot distinguish between singly- and multiply-charged ions of the same energy per unit charge. The filtering in an ExB probe is based purely on ion velocity. As it is independent of charge state, the multiply-charged ions, which are typically traveling faster than singly-charged ions, can be resolved and their fraction of total density calculated. The magnetic field in an ExB probe is typically supplied by permanent magnets, as the magnetic field supplied by permanent magnets does not vary with time and is not dependent on current or core material as in electromagnets. Two parallel plates, placed on either side of the ion path, supply the electric field. These act like a parallel-plate capacitor, providing an electric field dependent only on the distance between the plates and the applied voltage.

For an ion passing through a combined electric and magnetic field, the force experienced by the ion can be found by the equation:

$$
\vec{F}=q \vec{v} \otimes \vec{B}+q \vec{E}
$$


As is shown in Figure 6-1, the electric and magnetic fields as well as the ion velocity are perpendicular to each other in an ExB probe. The electric and magnetic fields are oriented so the forces each impart on the ion are in the opposite direction. This allows the equation for force to be simplified significantly, as the vector notation can be eliminated, leading to the equation:

$$
F=q(E-v B)
$$

In order for an ion to pass through the probe unimpeded, it must experience zero net force from the electric and magnetic forces. Solving for velocity, this yields the definition:

$$
v_{0}=\frac{E}{B} .
$$

This relation allows ions of a specific velocity to pass through the probe on a straight-line trajectory, as the magnetic Lorenz force will be equal and opposite to the applied electric force. Ions of other velocities will not be able to pass, as they will either be diverted too much by the magnetic field, or not enough to counteract the electric force.

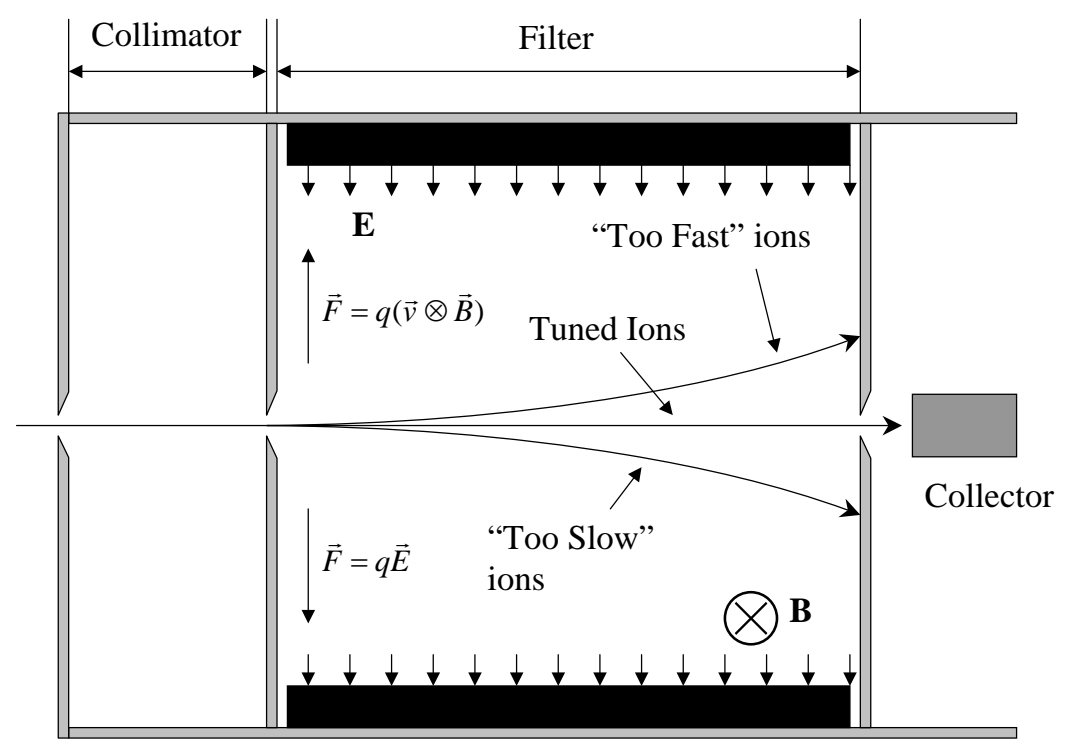

Figure 6-1: Major characteristics of an ExB probe 
The ExB probe is capable of a direct ion velocity distribution function measurement. This is justified using the flux of ions incident on the probe collector. For a given electrode voltage (electric field), the flux of ions is:

$$
\Phi=n_{v_{0}} v_{o}
$$

Eqn. 6-4

where $n_{v 0}$ is the density of ions with velocity $v_{0}$. Dividing the flux by the total ion density $n$ yields the ion flux per unit density as a function of the relative density of ions of velocity $v_{0}$ :

$$
\frac{\Phi}{n}=\frac{n_{v_{0}}}{n} v_{o}
$$

However, as the definition of the distribution function is the probability that ions of a given velocity will be found in the population, it can therefore be directly substituted for the relative density of ions. Thus:

$$
\Phi=n v_{0} f\left(v_{0}\right)
$$

This result shows that the flux of ions on the ExB detector is directly related to the ion velocity distribution function. Measured current is simply the flux multiplied by the collection area and ion charge, thus the ion velocity distribution function can then be directly found by:

$$
f\left(v_{0}\right)=\frac{I}{n q e A v_{0}}
$$

This result is important as it means the ExB probe is directly measuring the ion velocity distribution function, with the only necessary adjustment being the division of the probe current at all points by the velocity which corresponds to that current. The area of collection is difficult to determine in an ExB probe, so the constants $n, q, e$, and $A$ can be combined into a single normalization constant without affecting the nature of the data. A typical ExB probe trace is plotted in Figure 6-2, and clearly shows three peaks. Given the $300 \mathrm{~V}$ on the main anode for the operating condition displayed, singly charged xenon should have an energy of approximately $300 \mathrm{eV}$, doubly charged xenon $600 \mathrm{eV}$, and triply charged xenon $900 \mathrm{eV}$. These energies correspond to velocities of 21,000 m/s, 29,700 m/s, and 36,400 m/s for singly, doubly, and triply charged xenon, respectively. In the data in Figure 6-2, the velocities at the three peaks are $20,800 \mathrm{~m} / \mathrm{s}, 28,400 \mathrm{~m} / \mathrm{s}$, and 33,700 m/s. Each of these corresponds well to the predicted velocity of the three charge states of xenon. The difference between the observed ion velocities and those predicted by the 


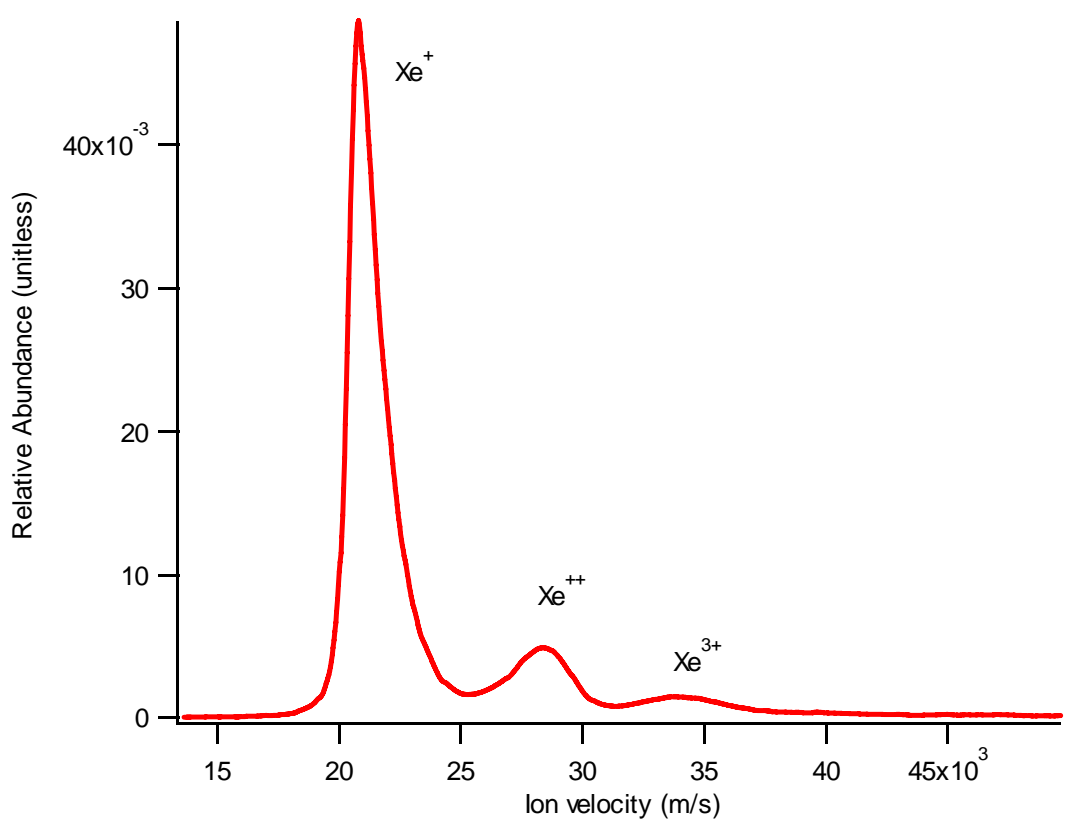

Figure 6-2: Typical normalized ExB probe distribution

thruster discharge voltage is due to the fact that ions are not always created at a point where they can be accelerated through the entire discharge potential of the thruster.

Determination of the ion velocity distribution function, then, can be accomplished by finding a functional fit for the currents detected by the ExB probe. There are two standard distribution functions which are used to describe ions in a plasma, with slightly different shapes and physical meanings. Both of these distribution functions are three-dimensional in nature. However, as the probe is in effect measuring the flux of ions on a flat plate, only the velocity component perpendicular to the collection area is important. Thus the one-dimensional forms of these distribution functions can be used. The first of the distributions is the Maxwellian distribution, as described by the equation:

$$
f(v)=\sqrt{\frac{\beta}{\pi}} e^{-\beta v^{2}} .
$$

The factor $\beta$ is calculated from the particle mass and temperature. The function as stated is centered on zero velocity. To account for bulk motion in the gas, the velocity $v$ can be replaced by $\left(v-v_{0}\right)$, thus shifting the maximum point of the distribution to the desired location in velocity space. The Maxwellian distribution is used extensively to describe the equilibrium properties of gases, where collisional processes between particles force the velocities to follow the distribution in Eqn. 2-23. This equilibrium condition is 
dependant on the atoms or molecules in the gas being in thermal equilibrium. A Hall thruster plume is not an equilibrium gas, however. Beam ions accelerated by the thruster cannot come to equilibrium before reaching the probe, and thus cannot be considered to have a purely Maxwellian distribution. Collisional processes still have an effect, however, and as such the Maxwellian distribution cannot be completely neglected.

A second distribution function that can be used is the Druyvesteyn function, as described by: ${ }^{34}$

$$
f(v)=N e^{-\beta v^{4}}
$$

This function is commonly used to describe a population of charged particles that are accelerated through a uniform electric field and undergoing collisions with other ions and neutral gas atoms. Due to the uniform acceleration the bulk of the ions will have velocities close to the average. Similarly to the Maxwellian distribution, the Druyvesteyn function can be adjusted for a population of ions with a bulk velocity by replacing $v$ with $\left(v-v_{0}\right)$. The Druyvesteyn function cannot fully describe the plasma in a Hall thruster, however. While collisional processes between particles in the beam are not sufficient to force the distribution to a Maxwellian form, they are also significant enough to prevent the function from being fully Druyvesteyn in nature. A combination of these must be used as neither of these models can fully describe the distribution function in a Hall thruster plume.

A method first used by $\mathrm{Kim}^{35}$ and later used by Hofer was to assume that prior to acceleration all ions had the same temperature. Thus when the ions were accelerated, the original ion temperature would determine the $\beta$ in the Maxwellian or Druyvesteyn models, and each population of ions in the distribution would have the same characteristic width. Due to the nature of the Maxwellian and Druyvestyn fits, however, the area under a distribution (and hence, the relative population of ions which that distribution describes) is directly proportional to the maximum height of the distribution and the width of the distribution. Width is described by $\beta$, and thus is the same for all populations of ions in the distribution. Thus the height is the only important factor in calculating the multiply charged ion fraction, and the need to perform a curve fit of multiple distribution functions (one for each peak in the distribution) is unnecessary. This method produced good results on an SPT-100 thruster as measured by Kim, with the multiply charged 
ion fractions showing good agreement with measurements taken using other methods by King $^{36}$ and Manzella. ${ }^{37}$

A complication arises in determination of the effect of multiply-charged ions on the collector, however. If the collector were a simple current collection plate or Faraday cup, the current seen by the probe could simply be divided by the charge state of the ions expected at a given velocity. Accounting for the increased current due to multiply charged ions, then, could be trivially performed by dividing the fraction calculated from the ExB distribution by the charge state of each measured species. Simple collection of probe current can be prohibitive due to the low incident current, however. Ion current densities typically in the regime of $10-50 \mathrm{~mA} / \mathrm{cm}^{2}$ are greatly reduced by both the filtering of the probe and the losses inherent in the fact that very few ions have the correct positions and angles with which they enter the probe to be detected, even if they are of the correct velocity and thus should be detected. To get around this, many ExB probes use charge-exchange multipliers to amplify the ion current directly. Charge exchange multipliers utilize either many plates or a hollow channel coated with semiconductor. Ions or electrons produce secondary electrons when they impact the entrance of the multipliers. These are accelerated towards the detector inside the multiplier, however as they travel they continually impact the walls or plates inside the multiplier. These impacts also produce secondary electrons, with the result that for a single ion or electron entering the probe, a great many secondary electrons are produced and detected. Typical maximum gains are on the order of $10^{8}$ amps per amp of incident current; thus up to one hundred million electrons are produced and detected for every incident ion or electron. The dynamics of chargeexchange multipliers make determination of whether the multiply-charged ions account for more current per ion difficult, however. In multipliers configured for ion detection, the entrance of the multiplier is typically biased to a large negative potential, intended to both accelerate the ions towards the multiplier and to accelerate secondary electrons produced deeper inside the multiplier. As this acceleration is electrostatic in nature, singly-charged ions will have a much lower velocity than multiply-charged ions as they impact the multiplier. The total effect on the collector current has been found to be that double ions cause current three times higher than singles and that triply charged ions cause current nine times higher than singles. Thus dividing the peak heights for the singles, doubles, and triples by 1,3 , and 9 , respectively, ${ }^{35}$ will give the correct relative abundance from which the multiply charged ion fraction can be calculated. 


\subsection{Ion Energy Error Analysis}

In an ideal probe, only ions with velocity $\vec{v}_{o}$ will pass through the probe and be detected. This is not possible, however, as the entry and exit orifices of the filter are of a finite size, and an ion that enters the probe with a velocity at an angle to the probe axis may be deflected back into line with ions that enter parallel to the axis, as shown in Figure 6-3.

Both the velocity and position of a non-optimal ion must be considered to determine if it will be detected by the probe. The position is important because an ion that may be deflected just enough not to pass if it were on axis may be deflected into the exit orifice of the filter and be detected. Velocity is important because an ion that is slightly faster or slower than the optimal velocity may not be deflected enough to prevent its detection. There are three components of velocity; parallel to the probe axis, parallel to $\vec{E}$, and parallel to $\vec{B}$. The velocity parallel to the probe axis is most important in determining if the ion will be detected; it is much larger than the other components and thus has much more of an effect on the magnetic force experienced than either of the other velocity components.

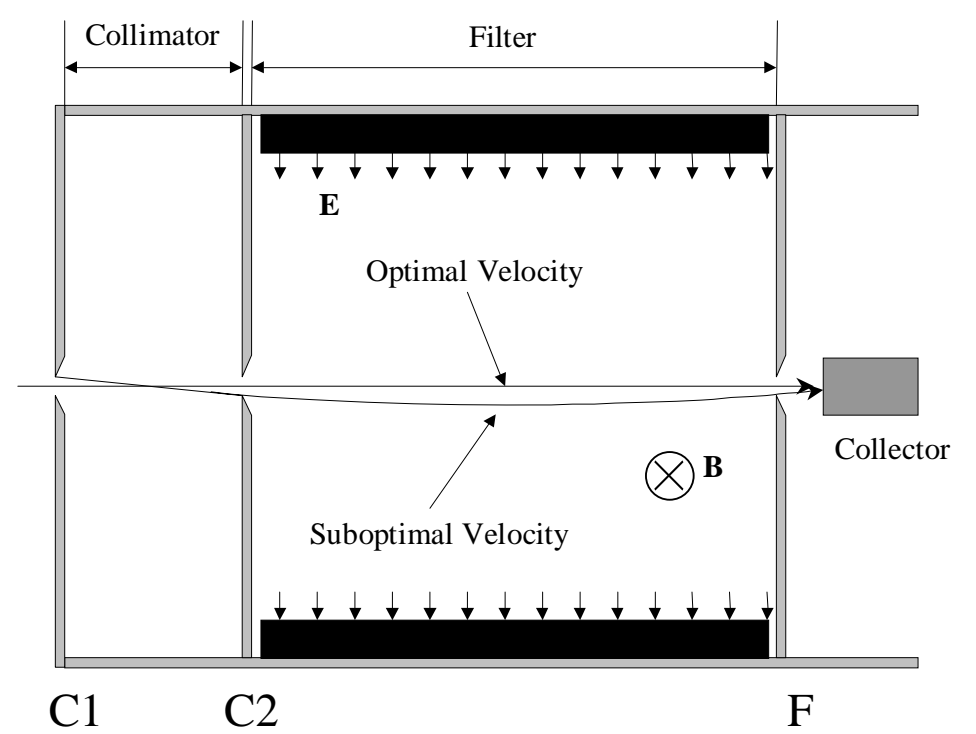

Figure 6-3: Schematic view of ExB probe showing detection of an off-axis ion. Plate $\mathrm{C} 1$ is the front plate of the probe, $\mathrm{C} 2$ is the filter entry or collimator exit plate, and $\mathrm{F}$ is the filter exit plate. 
In order to determine the off-axis angle and position with which an ion can enter the filter section of the ExB probe, the orifice size and spacing in the collimator section must be taken into account, as ions of only a small entry angle and off-axis distance can enter the filter. The largest distance off-axis the ion can enter is one-half the diameter of the orifice in plate C2; larger off-axis distances will impact plate C2 and never enter the filter. The largest entry angle allowed is determined by an ion entering at one side of the orifice in plate $\mathrm{C} 1$ and traveling unobstructed to the other side of the orifice in plate $\mathrm{C} 2$. The angle is given by the equation:

$$
\theta=\arctan \left(\frac{O_{C 1}+O_{C 2}}{2 l_{c}}\right)
$$

where $O_{c 1}$ is the diameter of the orifice in plate $\mathrm{C} 1, O_{c 2}$ is the diameter of the orifice in plate C2, and $l_{c}$ is the distance between plates C1 and C2.

Ions with components of velocity parallel to $\vec{B}$ will be detected only if that velocity component is small enough that the ion would pass through the filter if no electric or magnetic field were present, since velocity parallel to $\vec{B}$ is unaffected by either the electric or magnetic field. Thus the filter acts as a very large collimator, allowing ions with extremely small off-axis angles to pass, as the allowable angle (given no obstruction from the orifice in plate C2) will be the found by $\theta=\arctan \left(\left(O_{C 1}+O_{F}\right) / 2 l_{c}\right)$, where $O F$ is the diameter of the orifice in plate F.

Ions with components of velocity parallel to $\vec{E}$ are much harder to account for. Velocity along $\vec{E}$ can be changed by the fields in the filter, thus an ion with a total velocity different from $\vec{v}_{o}$ may be able to pass through the filter and be detected. In order to determine the range of ion velocities that allow such a trajectory, the acceleration of an ion under a nonzero sum of electromagnetic forces must be examined. For the purposes of this analysis, only the component of velocity parallel to the axis of the probe will be used to calculate magnetic force, as this component is nearly unaffected by either $\mathrm{E}$ or $\mathrm{B}$, and is much larger than the other components of velocity given a small entry angle. It can be assumed that the magnetic force is constant since the component of velocity used to calculate the magnetic force is unaffected by that force. Since the electric force does not change, the total acceleration can be assumed constant. Due to the combination of the assumed-constant acceleration due to the magnetic field and the constant acceleration 
due to the electric field, equation (2) can be combined with the general equation for the motion of a particle under constant acceleration:

$$
y=\frac{1}{2} a t^{2}+v_{p} t
$$

to give:

$$
y=\frac{q t^{2}}{2 m}\left(v_{l} B+E\right)+v_{p} t .
$$

where $y$ is the off-axis distance, $m$ is the mass of the ion, $v_{l}$ is the component of velocity parallel to the axis of the probe, $v_{p}$ is the component of velocity parallel to the electric field, and $t$ is time. The components of velocity are calculated using the maximum possible entry angle, as calculated in Eqn. 6-11. As the displacement of the ion at the end of the filter is of greatest importance, $t$ becomes the time required for the ion to traverse the filter section, which is expressed as the length of the filter divided by the velocity parallel to the probe axis. Thus, a substitution can be made into equation (6) to produce:

$$
y=\frac{q l^{2} B}{2 m v_{l}}+\frac{q l^{2} E}{2 m v_{l}^{2}}+\frac{v_{p} l}{v_{l}} .
$$

The equation can be further simplified by replacing the velocity components with expressions based on the total velocity:

$$
y=\frac{q l^{2} B}{2 m v \cos (\theta)}+\frac{q l^{2} E}{2 m v^{2} \cos ^{2}(\theta)}+\frac{v l \sin (\theta)}{v \cos (\theta)} .
$$

This change in variables removes the velocity dependence from the third term of the equation, which can now be trivially rearranged to:

$$
2 m \cos ^{2}(\theta)(y-l \tan (\theta)) v^{2}-q l^{2} B \cos (\theta) v-q l^{2} E=0 .
$$


The value of y to use in this equation is the total distance an ion can travel parallel to the electric field, assuming it enters at one side of the orifice in plate $\mathrm{C} 2$ and exits at the other side of the orifice in plate F. By substituting the average of the orifice sizes in plates C2 and F for $y$, equation (9) becomes a quadratic equation for $v$. Since $\theta$ can be positive or negative, maximum and minimum possible velocities can be calculated by changing the polarity of $\theta$. The maximum and minimum velocities are not necessarily the same distance from the optimal velocity, and must be calculated separately. This velocity spread is nontrivial to account for mathematically. Without extensive knowledge of the ion density and ion velocity, known with a sufficiently small resolution that the changes across the open area of the ExB collector plates are known, no mathematical compensation can be accomplished. As such, improvement of the energy resolution is the only way to accurately eliminate the spread in the distribution caused by this error.

\subsection{Probe Design}

The ExB probe utilized in the experiments reported here had a 5-cm collimator (distance between plates C1 and C2), and a 23-cm filter (distance between plates C2 and F). A photograph of the probe with major features labeled is in Figure 6-4. The orifices in plates C1 and C2 were $1.6 \mathrm{~mm}$ in diameter. The orifice in plate F was $2.4 \mathrm{~mm}$ in diameter. The expected ion energy resolution ranges above and below the tuned energy are plotted in Figure 6-5, and show that the resolution for a given probe design is a function of ion energy. All of the orifices had 90-degree chamfers on their downstream sides, to prevent ions from hitting the interiors of the orifices and unnecessarily reducing the measured current. The magnetic field was supplied by two 5-cm x 23-cm x 1.3-cm banks of NdFeB rare earth magnets. Rare earth magnets, with their very high field strength, were chosen to improve the resolution of the probe over that available with ceramic magnets. The magnets were separated by Teflon spacers, which also held and insulated the electrode plates from the structure of the probe. The current was read by a K and M Electronics model $7550 \mathrm{~m}$ channel electron multiplier. The multiplier was operated at a supply voltage of $-2330 \mathrm{~V}$, which correlated to a gain of up to $10^{8} \mathrm{~A}$ per amp of ion current collected. The casing of the probe as well as the three orifice plates were fabricated of magnetic stainless steel, to direct and focus the magnetic field while preventing oxidation as is typical of magnetic iron or mild steels. The output current of the electron multiplier was converted to a voltage signal via the current amplifier used with the RPA. The gains were 
much higher than in the RPA experiments, however, ranging from $10^{6}$ to $10^{8} \mathrm{~V}$ per amp, for a total system gain of $10^{14}$ to $10^{16}$ volts per amp. Voltages measured were on the order of $20 \mathrm{mV}$ at peak for gains of $10^{14}$ volts per amp (increasing roughly linearly with current amplifier gain), indicating the ion current seen by the probe was on the order of $0.2 \times 10^{-15}$ amps; 0.2 femtoamps, or approximately 3,000 singly-charged ions per second at peak. Tuning of the electrodes and thus the electric field in the probe was performed by use of a Keithley 2410 sourcemeter, which typically swept the voltage from zero to $300 \mathrm{~V}$ at a rate of $0.5 \mathrm{~Hz}$. The output of the sweep power supply was passed through a voltage divider with a grounded center tap, allowing one of the plates to be biased at half the total electrode voltage positive, and the other plate an equal distance below ground. The splitting of the sweep voltage was to prevent any deceleration effects in the probe. If one electrode was at a high potential while the other was grounded, the potential at the centerline of the probe (halfway between the electrode plates) is approximately half the applied voltage. This behaves as a potential hill similar to that in an RPA. In an ExB probe, however, deceleration of the ions will prevent proper filtering of velocity, as the ions are unnecessarily slowed as they enter the probe. Forcing the electrodes to equal voltages above and below ground allows the potential at the centerline of the probe to be at or near ground, thus eliminating any deceleration of the ions.

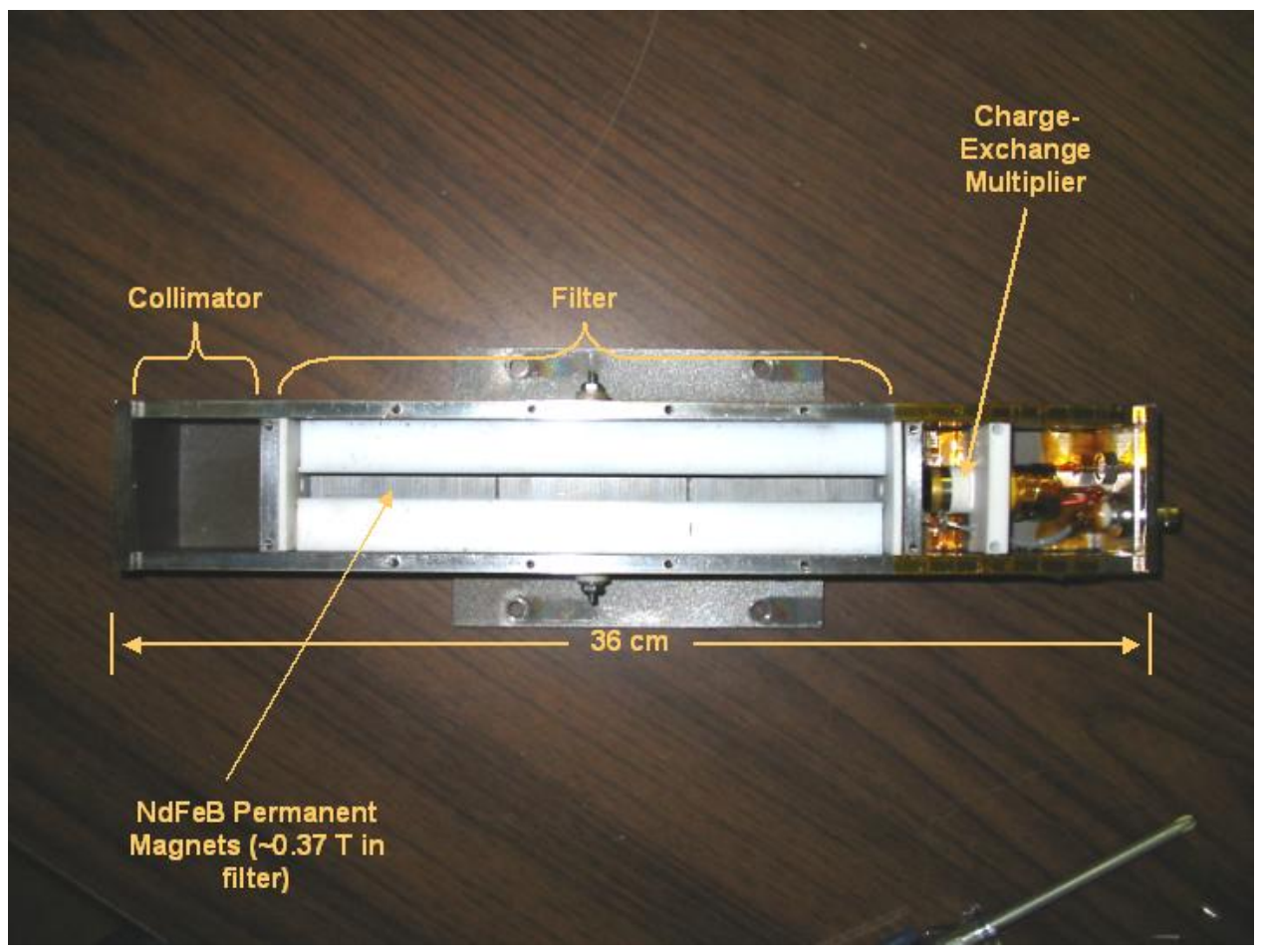

Figure 6-4: Photograph of the ExB probe with the top plate removed 


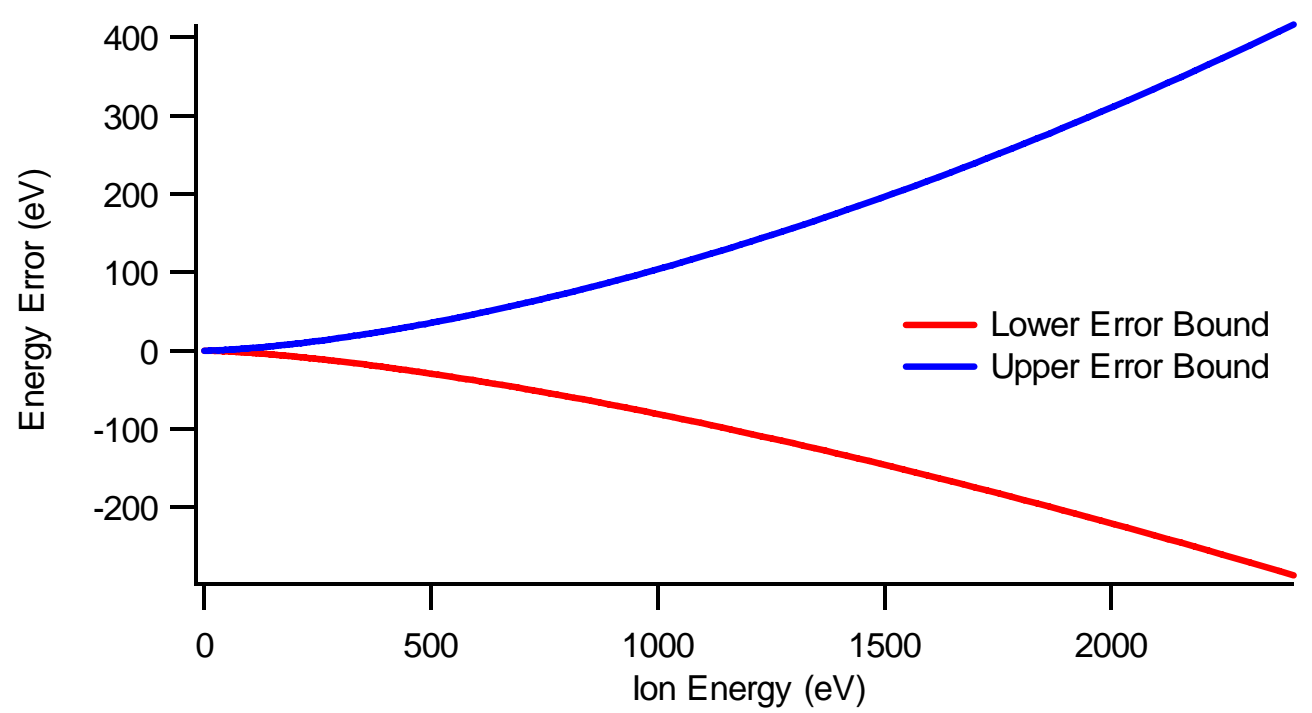

Figure 6-5: Resolution error of the ExB probe as a function of ion energy

One major change in the construction of the ExB probe over those used in previous studies was the addition of water-cooling. During development and initial testing of the probe, it was determined that the probe was becoming excessively hot. The excessive heat caused the ion peaks to start appearing at progressively lower velocities over the course of an experiment. This was due to the magnets inside the probe becoming too hot and becoming weaker. Permanent magnets exhibit a temporary loss of strength at high temperatures, and if they become too hot they will demagnetize permanently and have to be replaced. An additional problem with the probe heating was that one of the internal Teflon parts softened and slumped, nearly obscuring one of the collimator orifices. The solution to the heating problem was to enclose the probe in a water-cooled copper shroud, as shown in Figure 6-6. The shroud allowed the probe to be left in the thruster exhaust plume for long periods of time with no detrimental effect to the probe magnetic field or structural components. Addition of the copper water-cooled shroud caused significant amounts of sputtered copper deposition on the interior surfaces of the vacuum facility, which in turn required that the view ports in the facility be cleaned after each test. The thruster also gained a thin coating of copper on the front magnet poles and the cathode face. The coating did not affect thruster operation, and the coating was effectively removed through thruster operation without the ExB probe present in the facility.

Calibration of the ExB probe was accomplished by comparing the most probable ion energies calculated by the RPA analysis and the most probable ion energies obtained from the probe. As the probe 


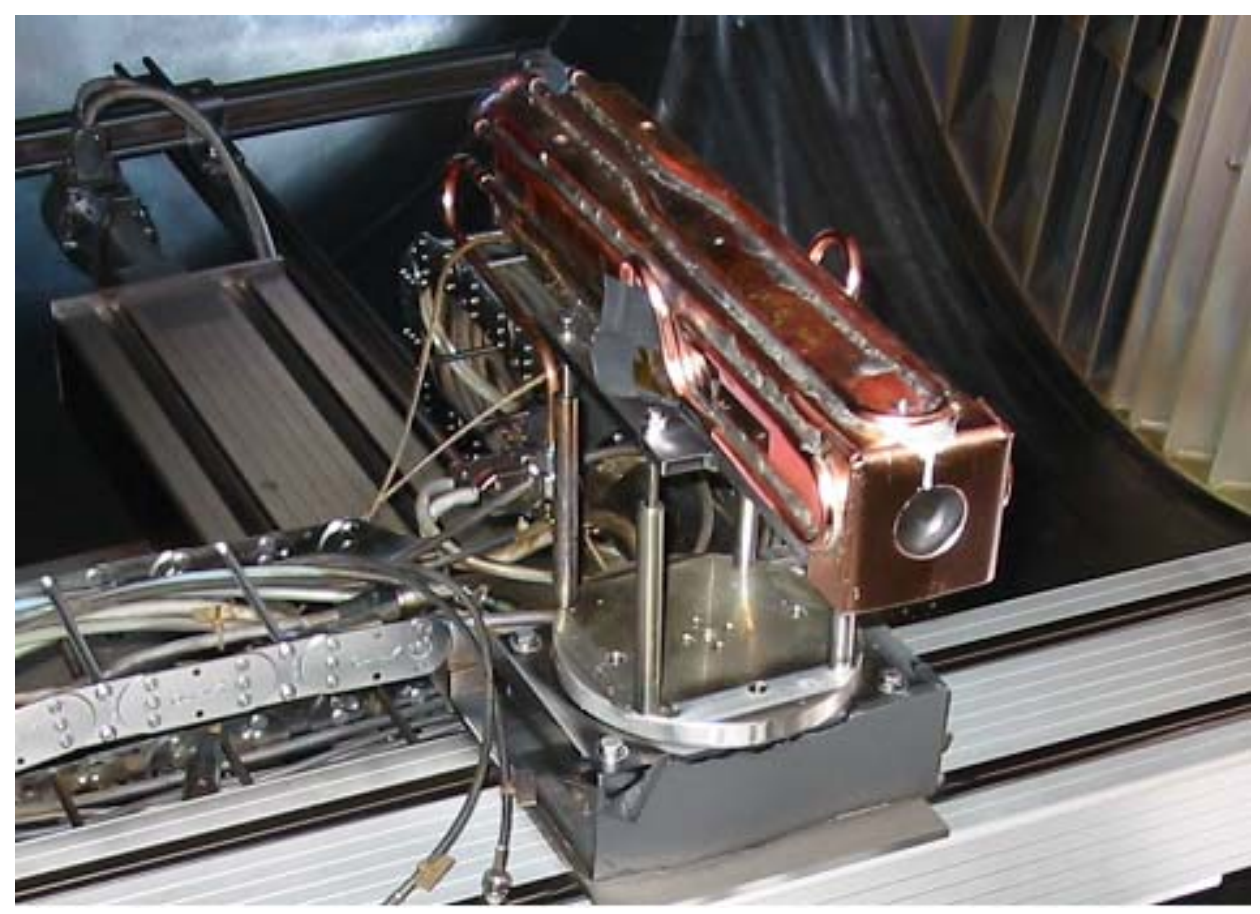

Figure 6-6: Photograph of the ExB probe in the water-cooled shroud

voltage is directly related to velocity and energy is proportional to the square of velocity, the square of the probe voltage is proportional to energy and the calibration must be performed with the square of the probe voltage as the independent variable. The ExB probe was calibrated using the raw RPA data, before compensation for plasma potential. This was done because the ExB probe is grounded and thus will accelerate the ions through the same potential as the RPA. However, as the ExB probe differentiates ions based on velocity and not through an electrostatic means, each peak in the distribution needs to be shifted by a different amount; the singly-charged ion peaks need to be shifted by an energy equivalent to the plasma potential, while the multiply-charged ions need to be shifted by energies equivalent to $q e V$; the plasma potential multiplied by the charge of the ions. This cannot be done simply by subtracting the plasma potential from all ion energies in the distribution, rather by subtracting the appropriate energy from each component of the distribution only after determining the charge state of each peak in the distribution.

Probe calibration was performed by using the most probable ion velocity from the ion energy measurements (assuming the peak of the ion energy distribution was the same as the peak of the singly charged xenon distribution) before compensation for plasma potential, and setting the velocity of the peak of the singly charged ion distribution in the ExB probe trace to the same velocity. The calibration was performed 45 times, one for each set of thruster operating conditions, thus generating 45 estimates for the 
calibration factor. This factor was calculated to be $225.659 \mathrm{~m} / \mathrm{s}$ per volt on the ExB electrode plates. The estimated error in the calibration factor is very low, despite the much larger uncertainty in the most probable ion energy measurements due to the fact that the calibration was repeated 45 times. The mean squared error of the calibration was $22.6 \mathrm{~m} / \mathrm{s} / \mathrm{V}$, leading to a $99 \%$ confidence interval with a width of $0.0067 \mathrm{~m} / \mathrm{s} / \mathrm{V}$. Thus despite the errors in the RPA measurements, the confidence in the calibration factor for the ExB probe was very good. The average calibration factor was used to calibrate the velocities on each ExB probe trace.

A typical set of ExB probe traces is in Figure 6-7, taken at $400 \mathrm{~V}$ on the anode and $4 \mathrm{mg} / \mathrm{s}$ of xenon. All of the ExB traces taken are plotted at the end of this chapter, in Figures 6-13 - 6-21. The ion velocities are not corrected for plasma potential. As the probe was grounded, the ions were accelerated into the probe similarly to the RPA. Multiply charged ions are affected to a greater degree by this acceleration, however, so the plasma potential cannot be compensated for merely by subtracting the plasma potential from the ion energies the probe were tuned to. The exact position of the peaks of ions of different charge states is unimportant for calculation of the multiply charged ion fraction, however, as the peak height is the only important parameter in this determination.

Each trace in Figure 6-7 therefore shows the velocity of ions as they passed through the probe filter. Three peaks are clearly visible in each trace. The spacing between peaks indicates that the first peak is singly charged xenon, the second doubly charged xenon, and the third peak triply charged xenon. This determination can be made because the velocity of the doubly charged xenon peak is roughly 1.4 times the 


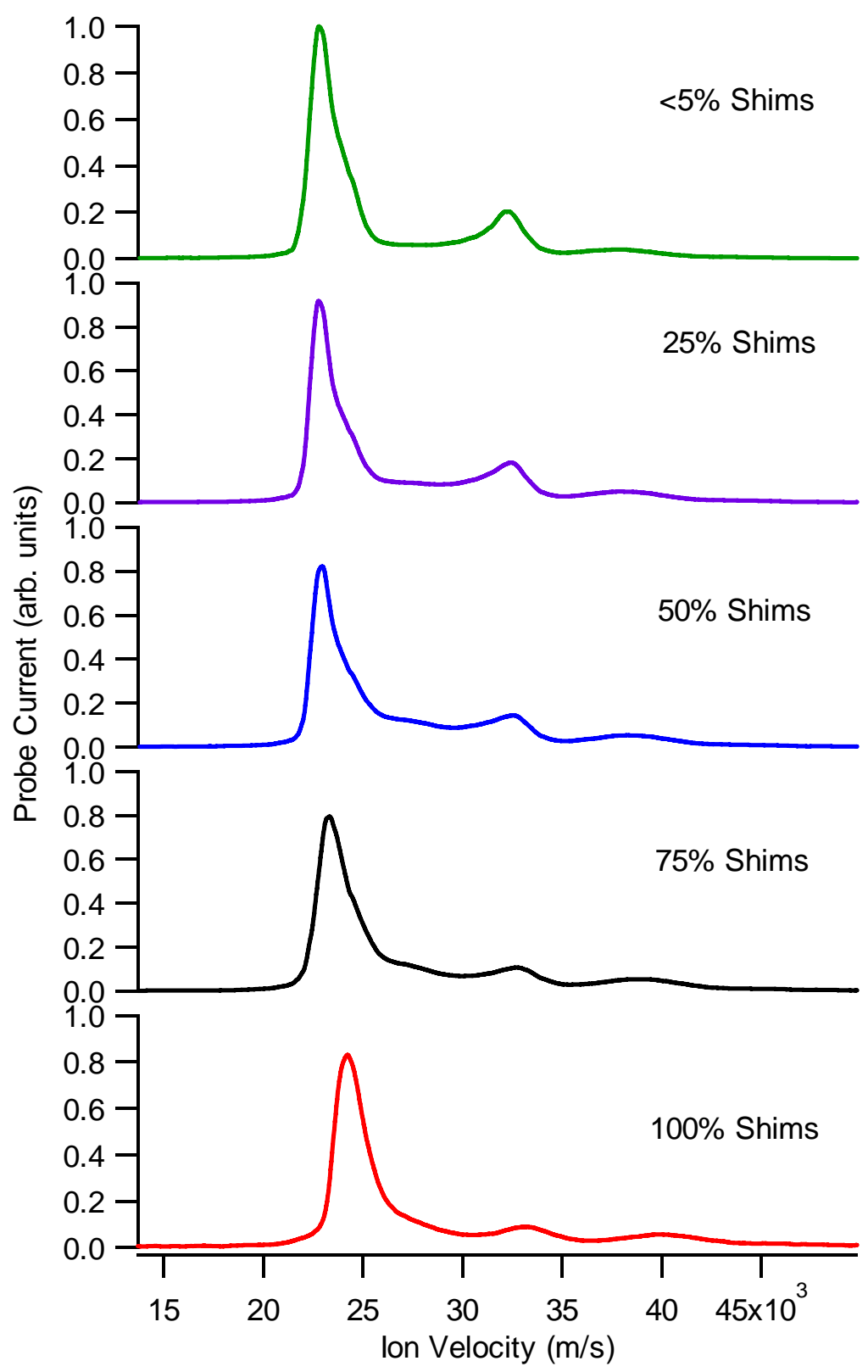

Figure 6-7: typical set of ExB probe traces taken as the level of shim current was varied. Data are for $400 \mathrm{~V}$ on the anode and $4 \mathrm{mg} / \mathrm{s}$ of xenon

velocity of the singly charged xenon peak. Doubly charged xenon, when accelerated through the same acceleration potential as a singly charged ion will have $\sqrt{2}$ (approximately 1.4) times the velocity as a singly charged xenon ion, so the peak is most likely to be doubly charged xenon. The velocity is far too high to be singly charged xenon produced and accelerated by a thruster at $400 \mathrm{~V}$, and thus must be doubly charged ions. The third peak in the distribution is similarly at approximately $\sqrt{3}$ times the velocity of the singly charged peak; the expected velocity of triply charged xenon, while being too fast to be either singly 
or doubly charged xenon. Xenon ions of higher charge states were not characterized. Extremely weak peaks were present at very high velocities at some thruster operating points that may have been quadruplecharged xenon, however the inconsistent appearance and small size of these peaks made comparison across all thruster operating points impossible and thus were neglected. Distribution peaks at velocities corresponding to higher charge states were not seen.

The data in Figure 6-7 show decreasing velocities at peak as discharge current was shifted from the shims to the anode. This is in agreement with the RPA measurements, which showed that the most probable velocity decreased as discharge current was shifted from the shims to the main anode. The shapes of the peaks are also slightly different. At the lower levels of current on the shims, the distributions are slightly narrower and therefore taller due to normalization. A more direct comparison of these traces is in Figure 6-8. The velocity axis has been expanded to show the behavior of the single and double xenon peaks more clearly. The most probable velocities of each peak show very similar behavior to the most probable energies measured by the RPA. The most probable velocity is highest when all discharge current is on the shims (high shim voltage), decreases as current is initially removed from the shims, then stabilizes as the shim voltage is reduced below the anode voltage. This provides further evidence for the ion acceleration being controlled by the highest potential present in the thruster. The shapes of the peaks indicate the acceleration is somewhat broadened when all of the discharge current was on the shims, as well. This is also in agreement with the data taken by the RPA. The similarities seen between the ExB and RPA energy distributions mean that the probes can be used interchangeably for information about most probable ion energy. 


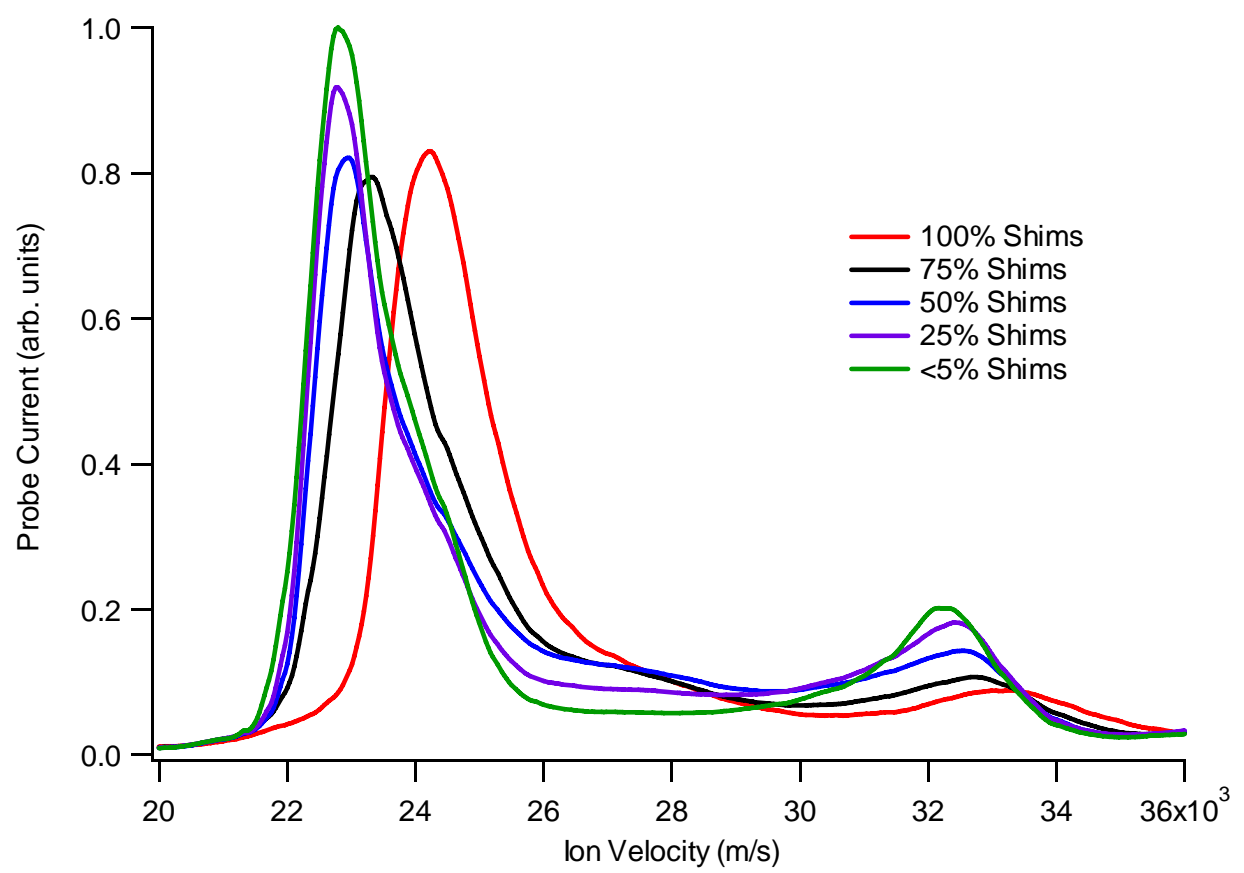

Figure 6-8: ExB probe traces taken with $400 \mathrm{~V}$ on the main anode and $4 \mathrm{mg} / \mathrm{s}$ of xenon.

The primary function of the $\mathrm{ExB}$ probe in the experiments reported here was to determine the multiply charged ion fraction in the thruster plume. Using the methods outlined in Section 6-1, the density fraction of ions in each charge state was calculated. These are plotted in Figures 6-9-6-11. Conclusions are difficult to make based on these data, however. Some of the combinations of anode voltage and mass flow show an increasing fraction of doubly charged and triply charged xenon at high shim voltages, while others show the inverse; reduced fractions of doubly and triply charged ions at high shim voltages. The multiply charged ion fractions calculated for the operating points with $6 \mathrm{mg} / \mathrm{s}$ of mass flow are much higher than those at lower mass flows, especially at low shim voltages. The anode voltage appears to have an effect on the multiply charged ion fraction, however. The fraction of doubles and triples in general increased as anode voltage was raised. This is consistent with measurements by previous researchers ${ }^{33}$ which showed the multiply charged ion fraction increases with anode voltage. 


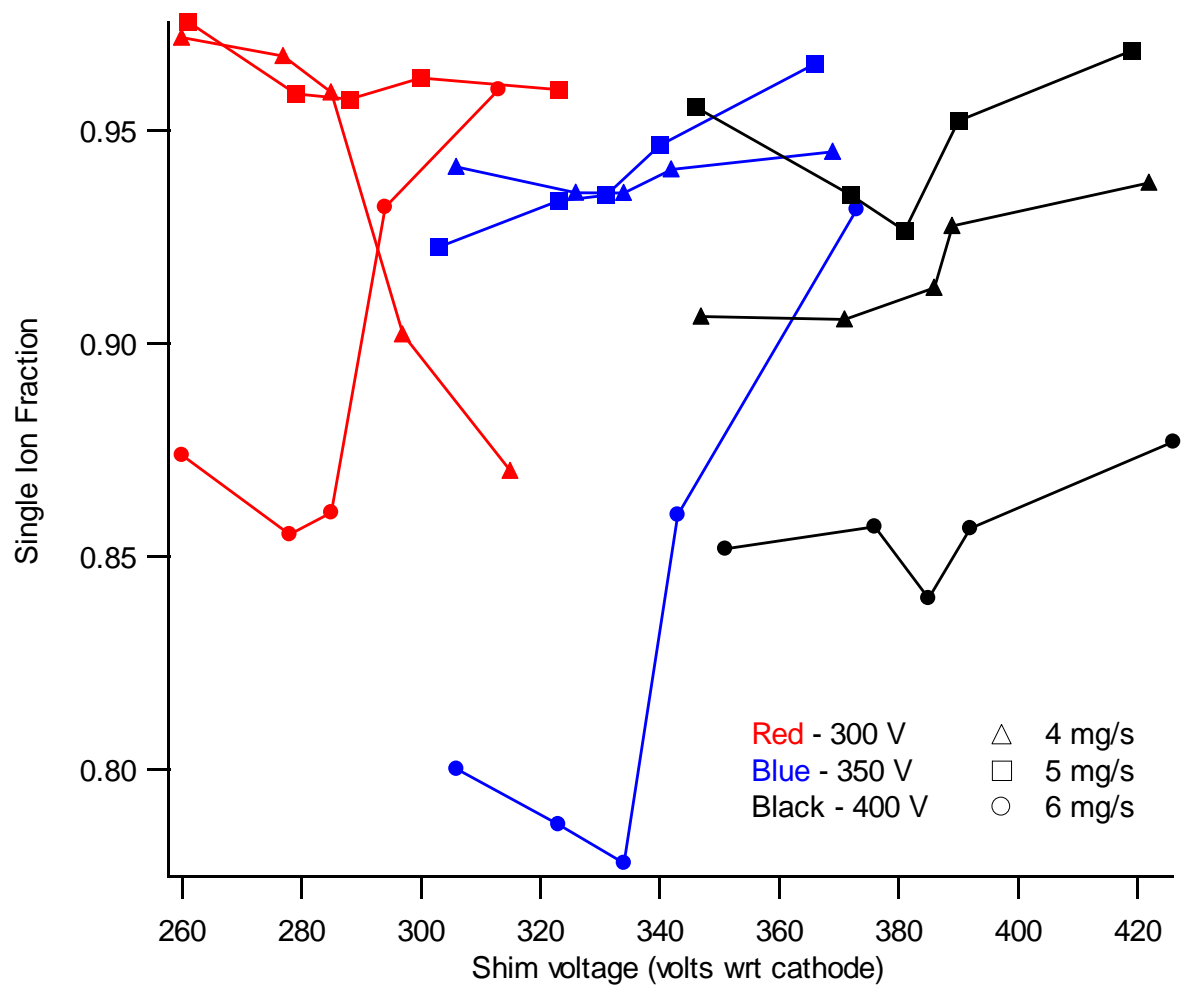

Figure 6-9: Singly charged ion density fraction for all thruster operating points examined. High shim voltages indicate high amounts of discharge current attachment on the shims

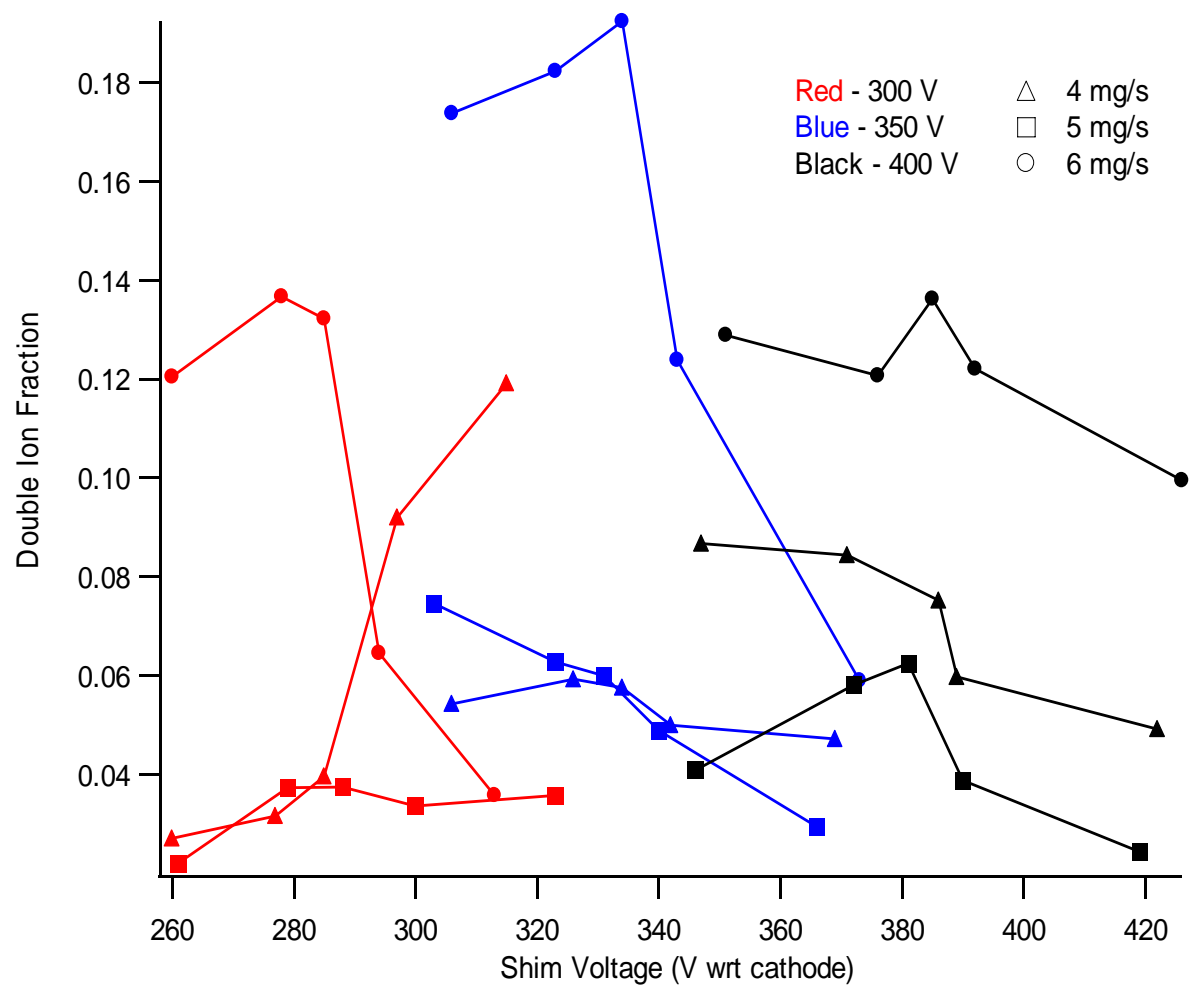

Figure 6-10: Doubly charged ion density fraction for all thruster operating points examined. High shim voltages indicate high amounts of discharge current attachment on the shims 


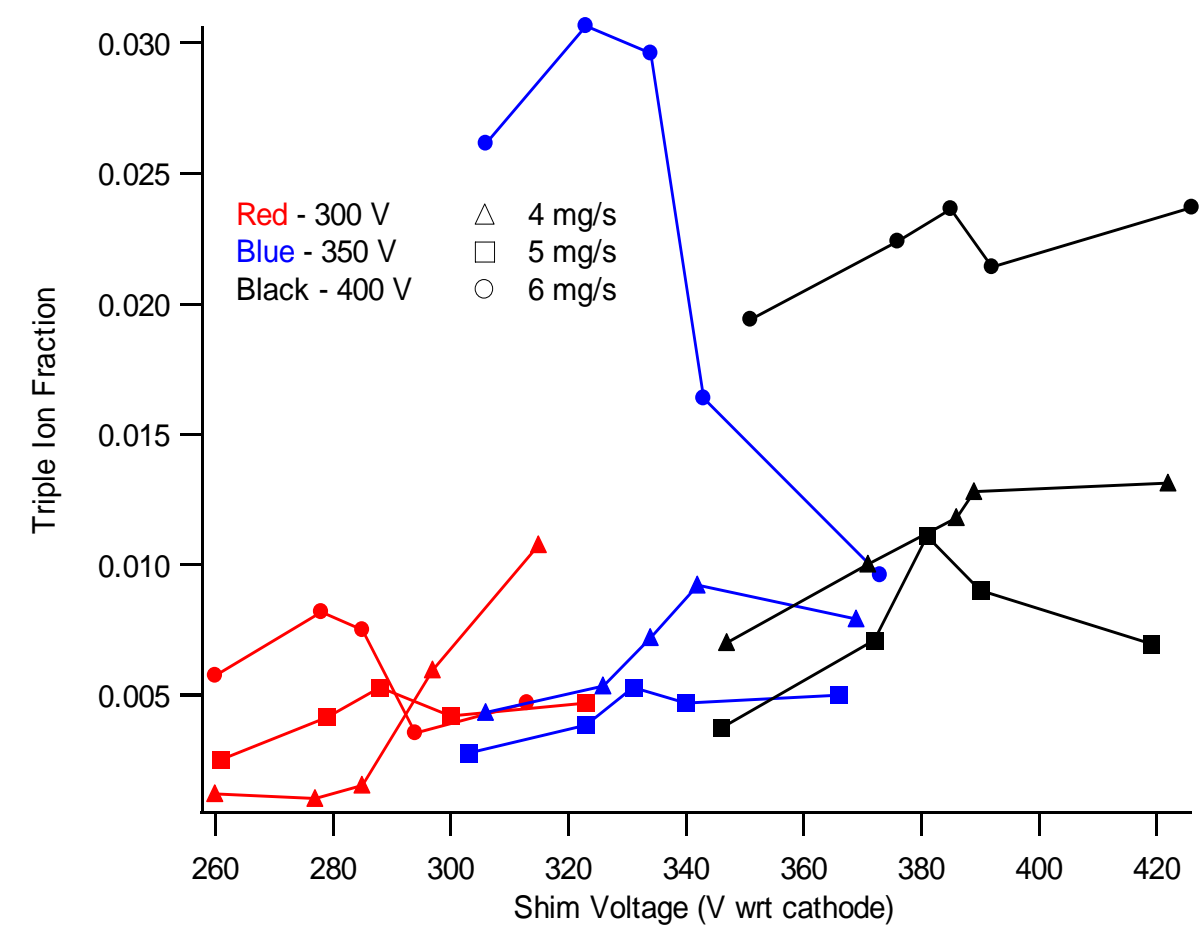

Figure 6-11: Triply charged ion density fraction for all thruster operating points examined. High shim voltages indicate high amounts of discharge current attachment on the shims

Application of the analysis of variance to each charge population provided more information about the nature of the factor effects on multiply charged ion fractions, and are in Tables 6-1 - 6-3. In all cases, the ion fraction was strongly influenced by mass flow. The dependence on anode voltage was much smaller than was theoretically expected, and in the case of the doubly charged ions was not a significant factor. The P-value of 0.055 for the effect of anode voltage on the singly charged ion fraction indicates the relationship is on the border between being significant and being insignificant. The triply charged ion fraction showed a strong dependence on the anode voltage, however. The level of discharge heating on the shims was not statistically significant as a factor in all three ion charge fractions. This is a very important conclusion to the future development of segmented electrode Hall thrusters. The discharge heating in a condensible propellant thruster can be shifted from shims to main anode without a significant change in multiply charged ion fraction.

Development of a confidence interval on the multiply charged ion fractions shows that the error is very large in the acquisition and analysis of these data through use of an ExB probe. The variances of each fraction, calculated to be $0.001,0.0008$, and $2.45 \times 10^{-5}$ for singles, doubles, and triples respectively, cause 
Table 6-1: ANOVA table for singly charged ion fraction

\begin{tabular}{|c|c|c|c|c|c|}
\hline $\begin{array}{c}\text { Experimental } \\
\text { Factor }\end{array}$ & Sum of Squares & $\begin{array}{c}\text { Degrees of } \\
\text { Freedom }\end{array}$ & Mean Squared & $\mathrm{F}_{0}$ & P-Value \\
\hline Anode Voltage & 0.0065 & 2 & 0.0033 & 3.1423 & 0.055 \\
\hline Mass Flow & 0.0656 & 2 & 0.0328 & 31.5337 & $1.22 \times 10^{-8}$ \\
\hline Shim Level & 0.0069 & 4 & 0.0017 & 1.6690 & 0.179 \\
\hline Error & 0.0400 & 36 & 0.0010 & & \\
\hline Total & 0.1165 & 44 & & & \\
\hline
\end{tabular}

Table 6-2: ANOVA table for doubly charged ion fraction

\begin{tabular}{|c|c|c|c|c|c|}
\hline $\begin{array}{c}\text { Experimental } \\
\text { Factor }\end{array}$ & Sum of Squares & $\begin{array}{c}\text { Degrees of } \\
\text { Freedom }\end{array}$ & Mean Squared & $\mathrm{F}_{0}$ & P-Value \\
\hline Anode Voltage & 0.0034 & 2 & 0.0017 & 2.0860 & 0.139 \\
\hline Mass Flow & 0.4950 & 2 & 0.0248 & 30.2889 & $1.93 \times 10^{-8}$ \\
\hline Shim Level & 0.0065 & 4 & 0.0016 & 1.9917 & 0.117 \\
\hline Error & 0.0300 & 36 & 0.0008 & & \\
\hline Total & 0.0888 & 44 & & & \\
\hline
\end{tabular}

Table 6-3: ANOVA table for triply charged ion fraction

\begin{tabular}{|c|c|c|c|c|c|}
\hline $\begin{array}{c}\text { Experimental } \\
\text { Factor }\end{array}$ & Sum of Squares & $\begin{array}{c}\text { Degrees of } \\
\text { Freedom }\end{array}$ & Mean Squared & $\mathrm{F}_{0}$ & P-Value \\
\hline Anode Voltage & 0.0006 & 2 & 0.0003 & 12.72 & $6.64 \times 10^{-5}$ \\
\hline Mass Flow & 0.0011 & 2 & 0.0006 & 23.10 & $3.51 \times 10^{-7}$ \\
\hline Shim Level & $5.29 \times 10^{-5}$ & 4 & $1.32 \times 10^{-5}$ & 0.54 & 0.71 \\
\hline Error & 0.0009 & 36 & $2.45 \times 10^{-5}$ & & \\
\hline Total & 0.0027 & 44 & & & \\
\hline
\end{tabular}

the uncertainty in the data to be quite large. For singly charged xenon, the $95 \%$ confidence interval has a width of 0.065 , or nearly $7 \%$ of the total ion population and $6.7-8.4 \%$ of the calculated fraction of singly charged ions. The width of the confidence interval on the doubly charged xenon is 0.058 , nearly $6 \%$ of the total ion population. This uncertainty is $30-265 \%$ of the calculated quantity of doubly charged ions. For triply charged ions, the width of the confidence interval is $1 \%$ of the total ion population, or between 33 and $960 \%$ of the measured fraction of triply charged ions.

An alternative to separate examination of each species is to develop an average charge for ions in the thruster discharge. The average charge can be trivially calculated from the equation:

$$
\bar{q}=\sum_{i=1}^{Z} q_{i} F_{i}
$$

As the highest charge state measured was triply charged ions, the value of $\mathrm{Z}$ is 3 . The average charge at all operating points examined is in Figure 6-12. The results are very similar in appearance to the doubly charged ion fraction, and show many of the same trends. As in the fractions for each charge state of ions, the trends as shim voltage is changed are somewhat indeterminate. The general conclusion from the ion 


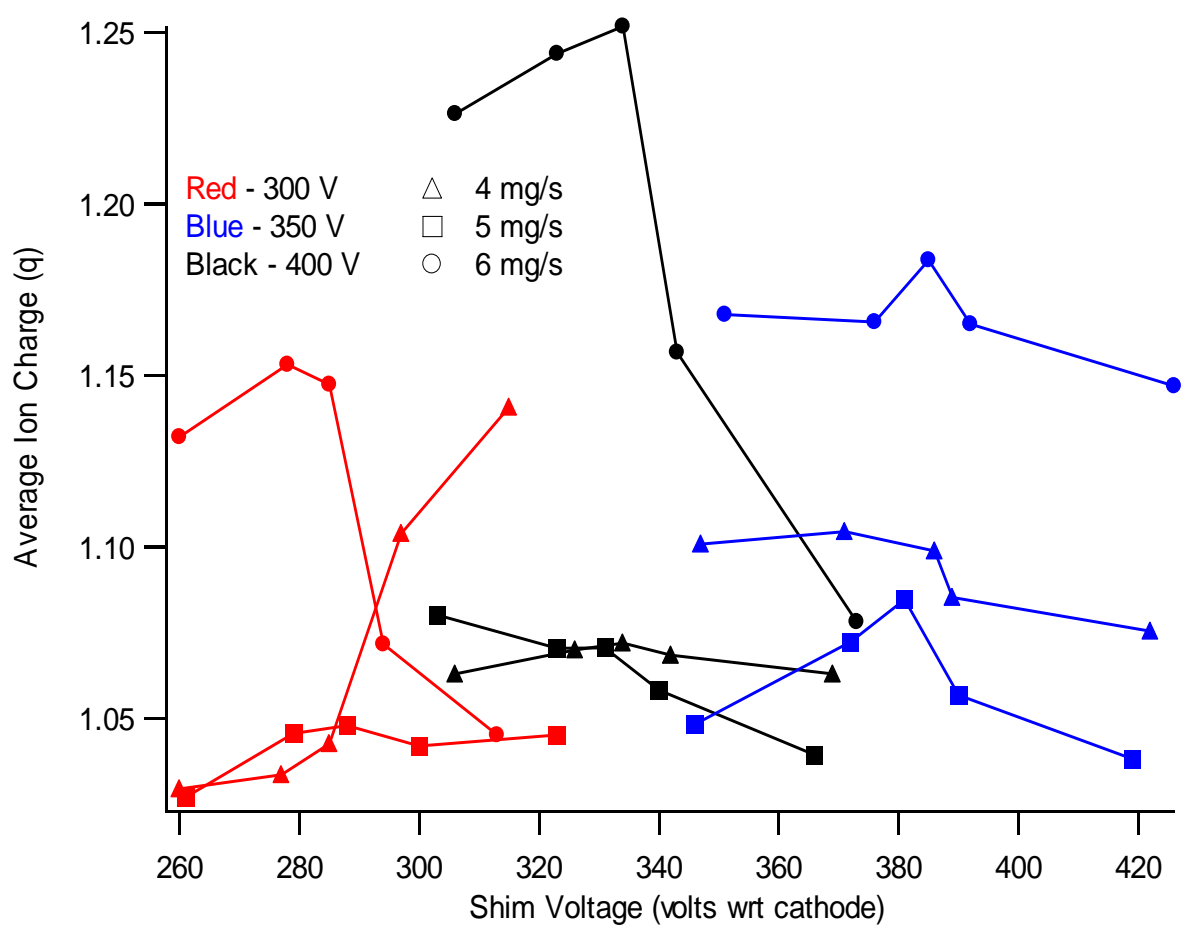

Figure 6-12: Average ion charge as a function of all three experimental factors. High shim voltage for indicates a higher fraction of discharge current heating on the shims.

fractions, that multiply charged ion fraction increases with anode voltage, is apparent in the average charge, as for the most part the average charge at the various operating points increases with anode potential.

Determination of the statistically significant factors in the average ion charge is different than finding the significant factors for each population. The average charge is a composite measurement of all charge states, thus finding the statistically significant factors is therefore applicable to the entire population of ions and not merely to one charge state. After the analysis of variance in Table 6-4, it was found that the mass flow is the most significant factor in average ion charge, with anode voltage being significant but not as large as mass flow. Shim level, with a $24.5 \%$ certainty in the null hypothesis, was determined not to be a significant factor in average ion charge. Thus much the same conclusion is reached by analyzing the average ion charge as was reached by analysis of each component separately; mass flow has the largest effect on the multiply charged ion fraction, anode voltage is still significant but not to the same degree as mass flow, and shim level was not a significant factor. Using the mean squared error generated by the analysis of variance, the 95\% confidence interval on the average ion charge has a width of 0.07 fundamental units of charge. This is between 5.9 and $7.2 \%$ of the calculated average ion charges, slightly 
Table 6-4: ANOVA table for average ion charge

\begin{tabular}{|c|c|c|c|c|c|}
\hline $\begin{array}{c}\text { Experimental } \\
\text { Factor }\end{array}$ & Sum of Squares & $\begin{array}{c}\text { Degrees of } \\
\text { Freedom }\end{array}$ & Mean Squared & $\mathrm{F}_{0}$ & P-Value \\
\hline Anode Voltage & 0.0109 & 2 & 0.0055 & 4.158 & 0.024 \\
\hline Mass Flow & 0.0839 & 2 & 0.0419 & 31.994 & $1.034 \times 10^{-8}$ \\
\hline Shim Level & 0.0075 & 4 & 0.0019 & 1.426 & 0.245 \\
\hline Error & 0.0472 & 36 & 0.0013 & & \\
\hline Total & 0.1495 & 44 & & & \\
\hline
\end{tabular}

smaller than the confidence interval on the singly charged ion population and much smaller than the interval on the doubly and triply charged populations.

The uncertainties calculated on the multiply charged ion fractions are very large, and complicate further analysis of other thruster parameters as a function of multiply charged ion fraction. Some of this is inherent to the measurement method; an error of a few percent of the total ion population can be several times the fraction of triply charged ions. There are some fundamental limits to the analysis method utilized, however, which limit the accuracy of an ion charge fraction measurement. The only way to truly determine the ratios of each charge state of ions is to find a function that describes the shape of the distribution function exactly. This cannot be trivially done on the data reported here, however, as the uncertainty in the ion energies allowed to pass through the ExB probe is too large. Broadening of the distribution function caused by the energy resolution error makes finding a functional fit impossible, as the function will classify some ions of a given charge state as belonging to a different charge state. Increased resolution of the probe would allow for much finer resolution of the ion population peaks, and make either a functional fit or a more accurate measurement by peak height possible.

As it was determined that shifting current from the shims to the main anode was not a significant factor in the average ion charge, this factor can be eliminated from the statistical model, and thus the new experimental model for the average ion charge can be expressed as a two-factor model with five replicates. This allows resolution of the interaction between the anode voltage and mass flow effects, and also provides a single average number for the average ion charge at every combination of anode voltage and mass flow. The new analysis of variance, based on this reduced-factor model, is in Table 6-5. As in the analysis performed with the full three-factor model, the mass flow is the most significant factor in the average ion charge, and hence, the multiply charged ion fraction. Anode voltage is still a significant factor, but similarly to the analysis performed earlier, it is a much smaller contributor than mass flow. The 
interaction between anode voltage and mass flow is not significant, with a P-value of over $13 \%$. The 95\% confidence interval on the average charge calculated through this method is $0.032 q$, a much smaller amount than that calculated with the three-factor model.

Table 6-5: ANOVA table of average ion charge under the two-factor model approximation

\begin{tabular}{|c|c|c|c|c|c|}
\hline $\begin{array}{c}\text { Experimental } \\
\text { Factor }\end{array}$ & Sum of Squares & $\begin{array}{c}\text { Degrees of } \\
\text { Freedom }\end{array}$ & Mean Squared & $\mathrm{F}_{0}$ & P-Value \\
\hline Anode Voltage & 0.0109 & 2 & 0.0055 & 4.38 & 0.019 \\
\hline Mass Flow & 0.0839 & 2 & 0.0420 & 33.67 & $2.23 \times 10^{-9}$ \\
\hline Interaction & 0.0098 & 4 & 0.0025 & 1.97 & 0.134 \\
\hline Error & 0.0449 & 36 & 0.0012 & & \\
\hline Total & 0.1495 & 44 & & & \\
\hline
\end{tabular}




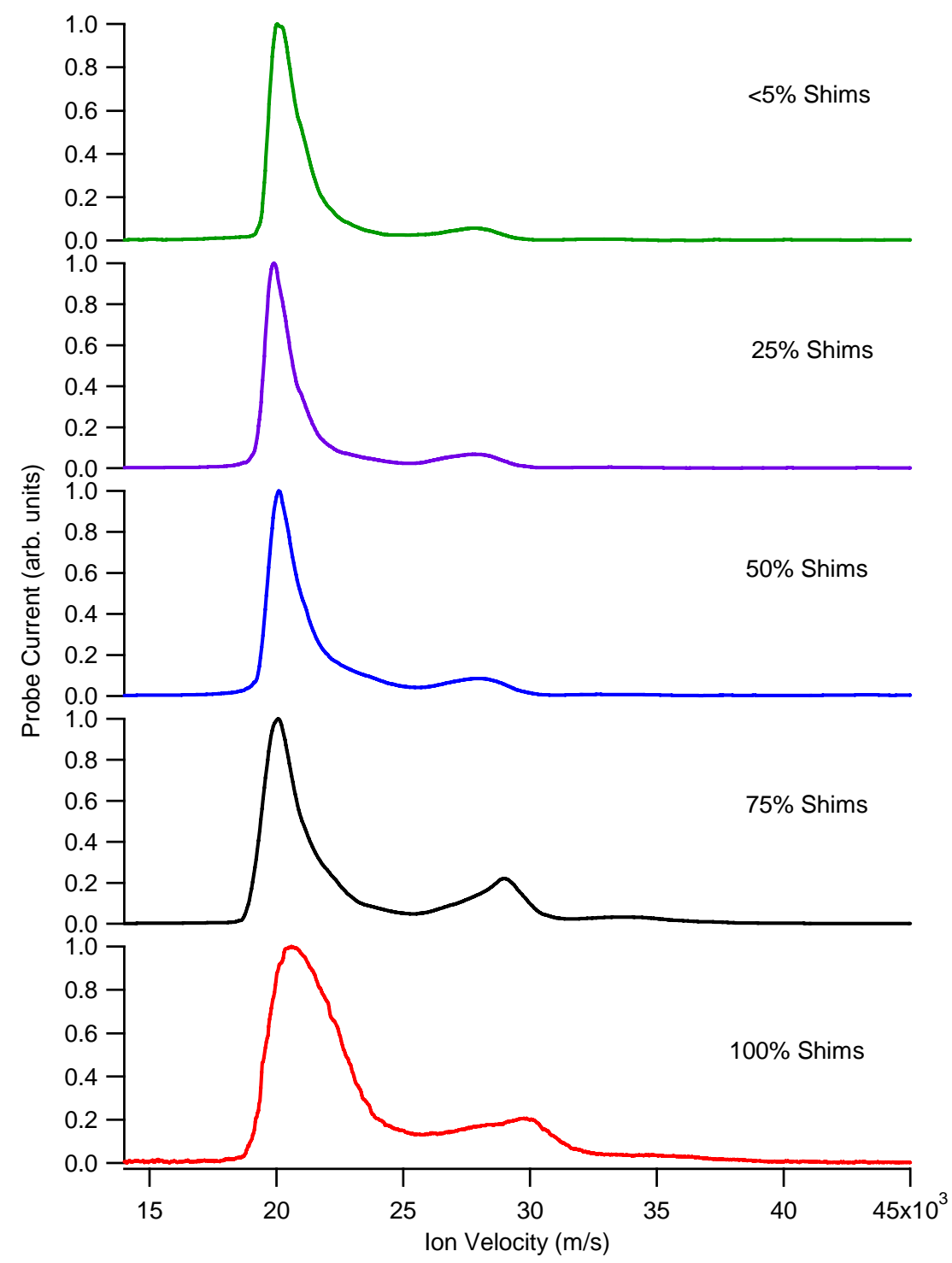

Figure 6-13: ExB probe traces taken as the level of shim current was varied. Data are for $300 \mathrm{~V}$ on the anode and $4 \mathrm{mg} / \mathrm{s}$ of xenon 


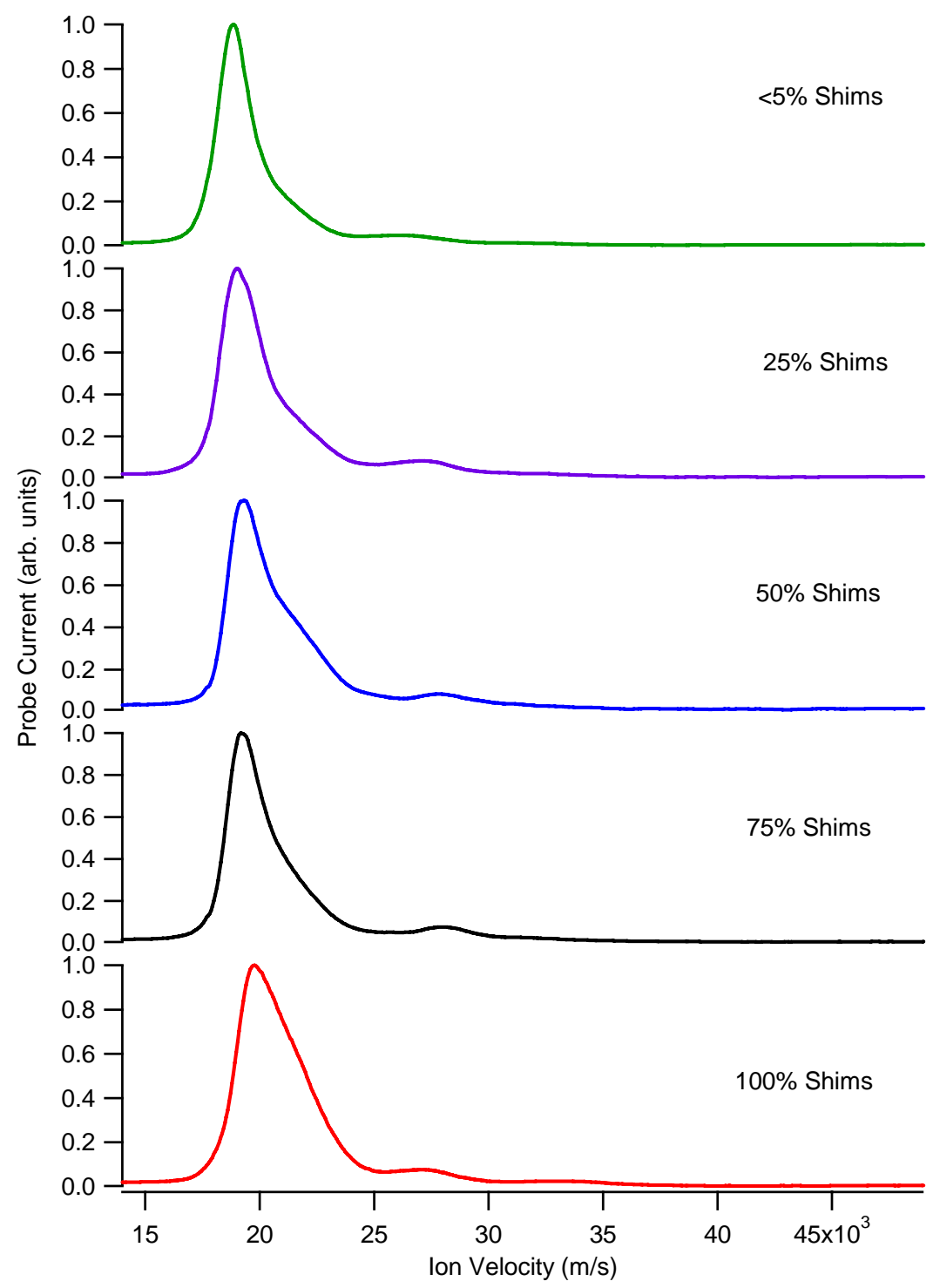

Figure 6-14: ExB probe traces taken as the level of shim current was varied. Data are for $300 \mathrm{~V}$ on the anode and $5 \mathrm{mg} / \mathrm{s}$ of xenon 


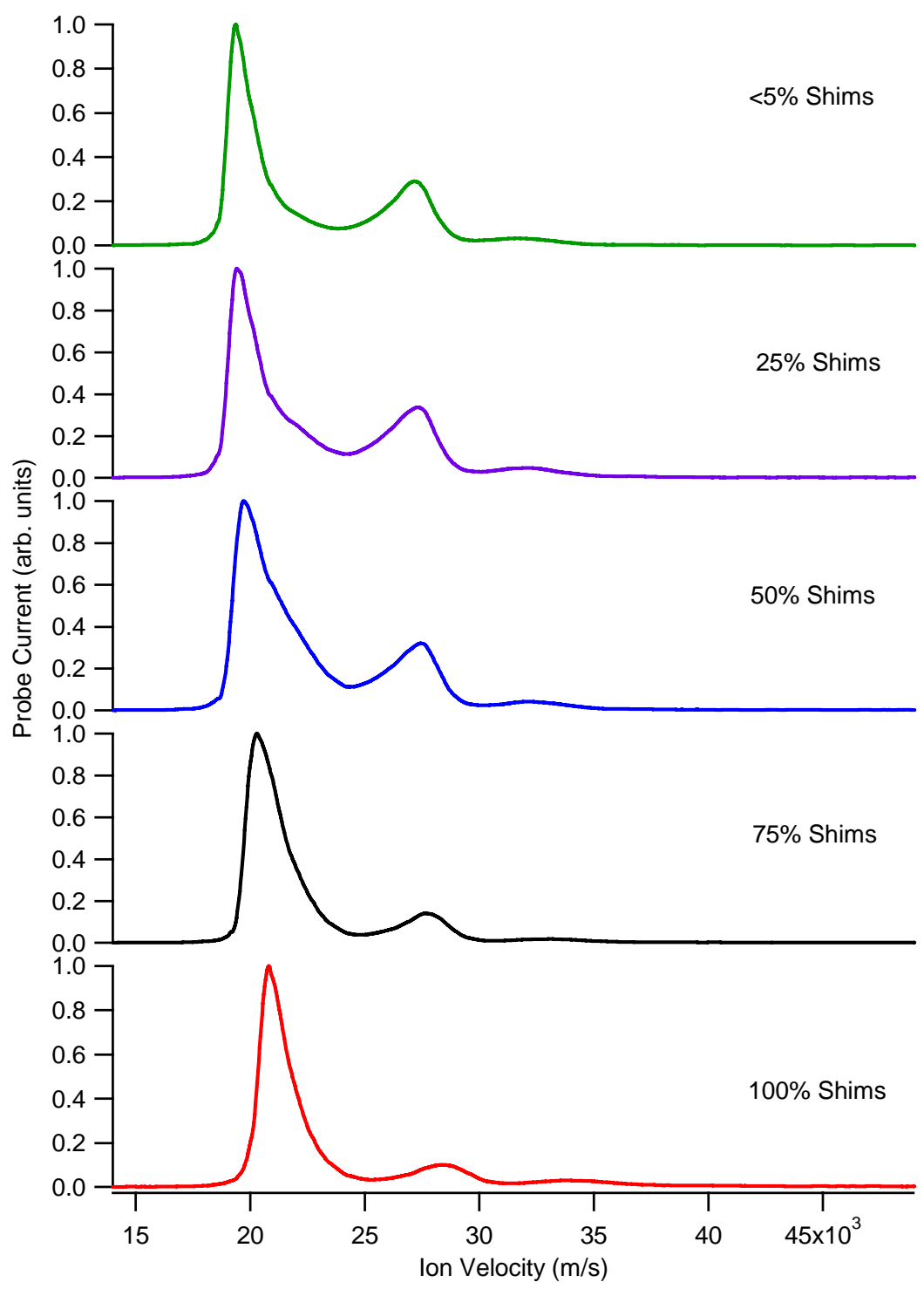

Figure 6-15: ExB probe traces taken as the level of shim current was varied. Data are for $300 \mathrm{~V}$ on the anode and $6 \mathrm{mg} / \mathrm{s}$ of xenon 


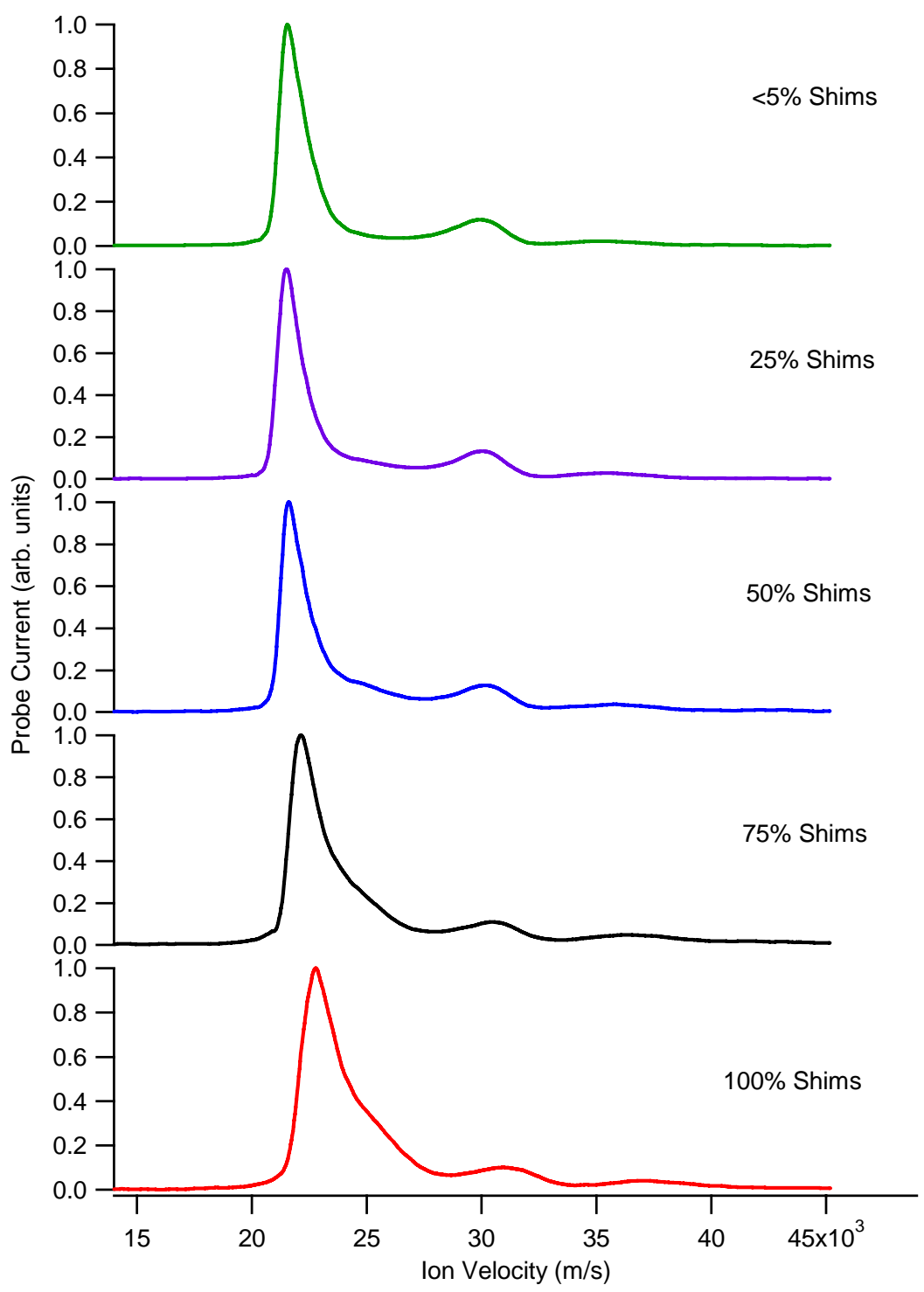

Figure 6-16: ExB probe traces taken as the level of shim current was varied. Data are for $350 \mathrm{~V}$ on the anode and $4 \mathrm{mg} / \mathrm{s}$ of xenon 


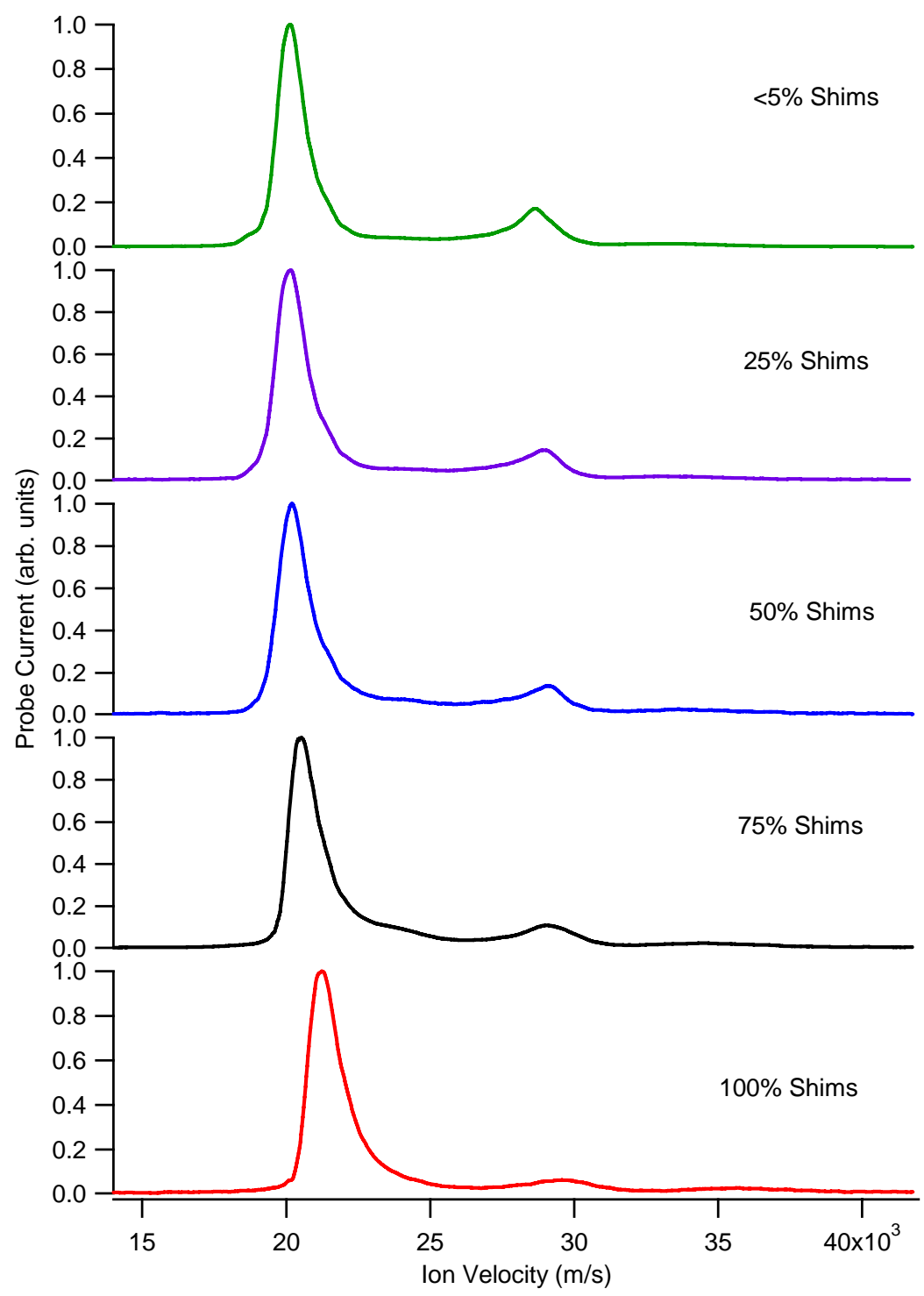

Figure 6-17: ExB probe traces taken as the level of shim current was varied. Data are for $350 \mathrm{~V}$ on the anode and $5 \mathrm{mg} / \mathrm{s}$ of xenon 


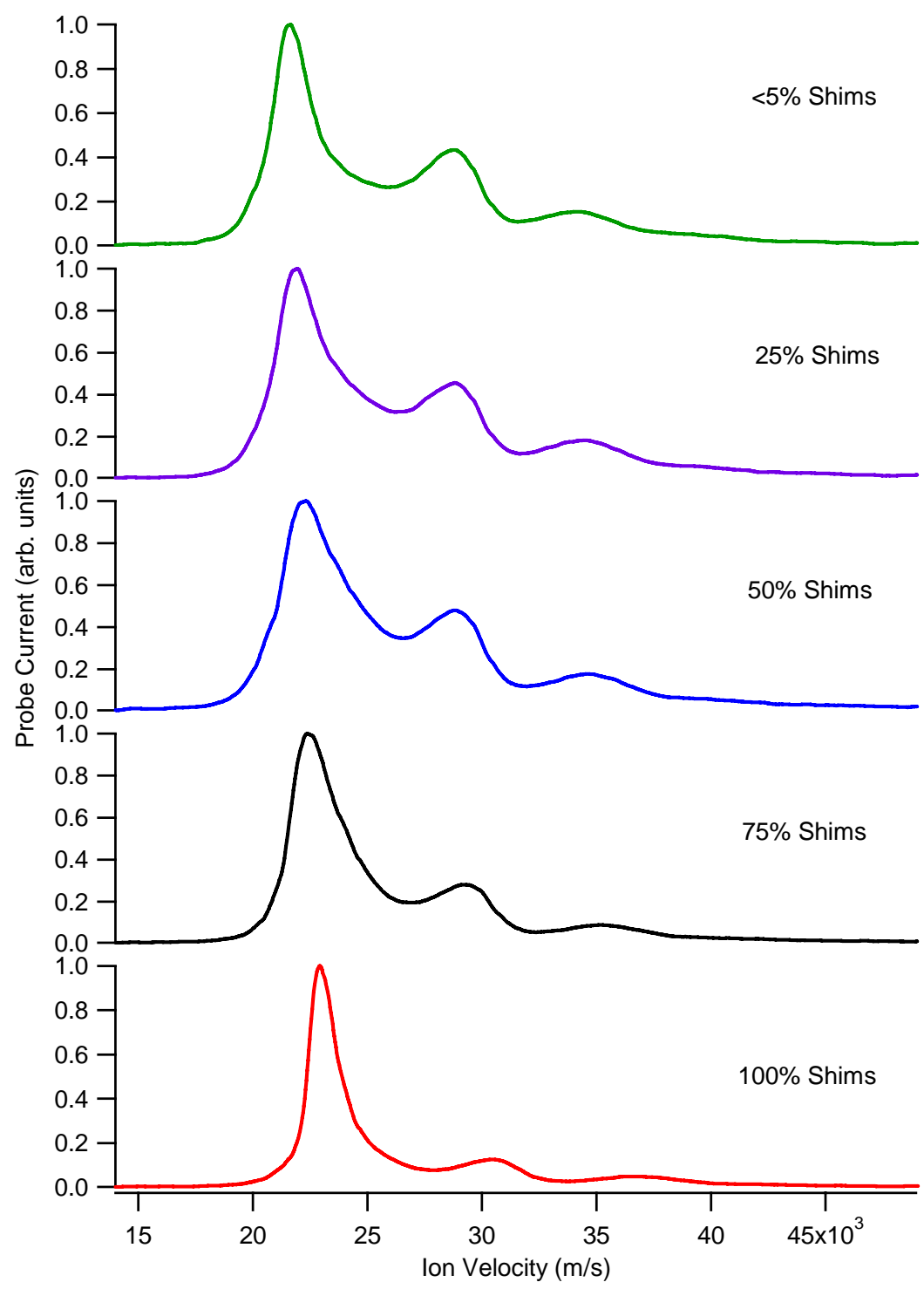

Figure 6-18: ExB probe traces taken as the level of shim current was varied. Data are for $350 \mathrm{~V}$ on the anode and $6 \mathrm{mg} / \mathrm{s}$ of xenon 


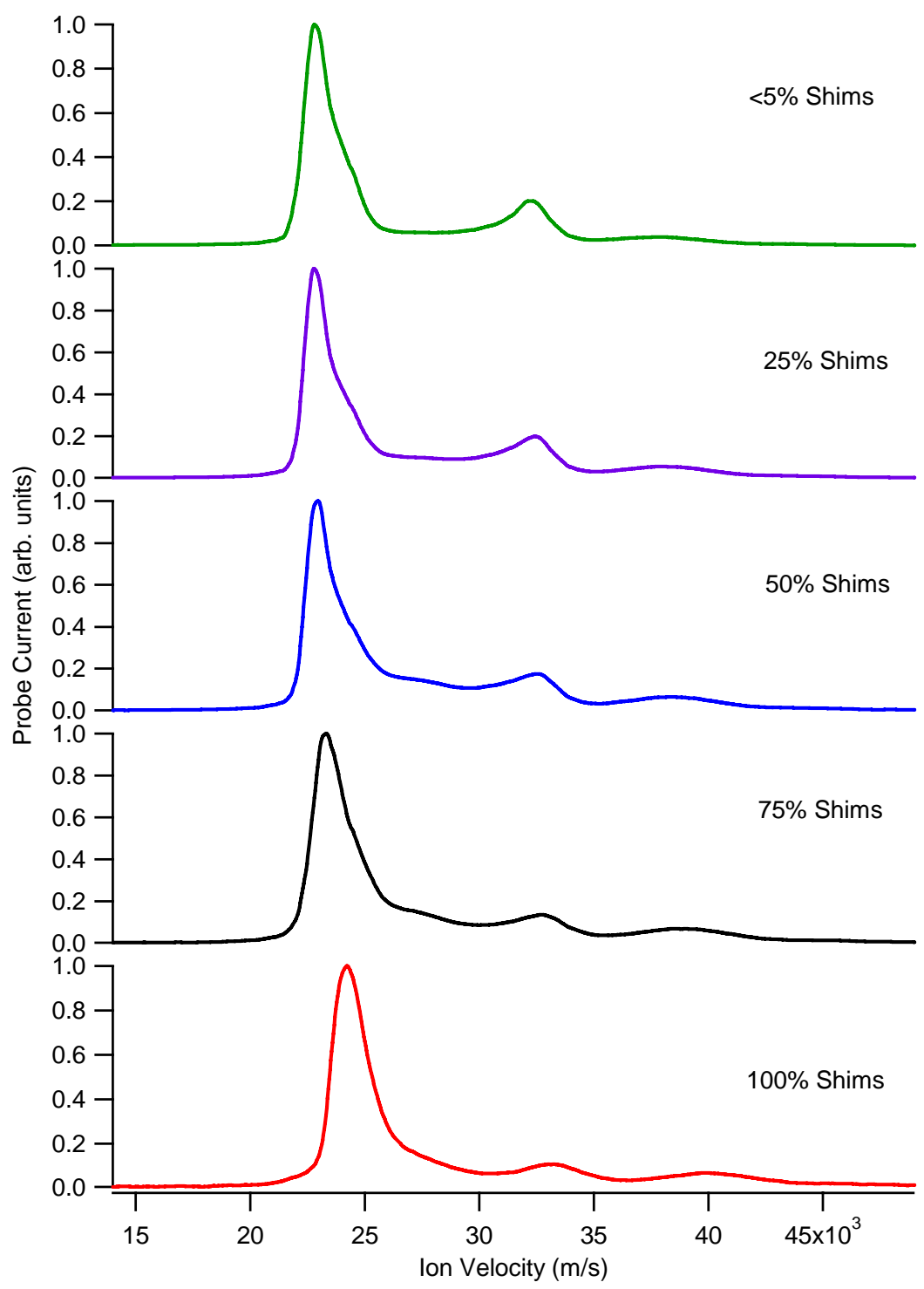

Figure 6-19: ExB probe traces taken as the level of shim current was varied. Data are for $400 \mathrm{~V}$ on the anode and $4 \mathrm{mg} / \mathrm{s}$ of xenon 


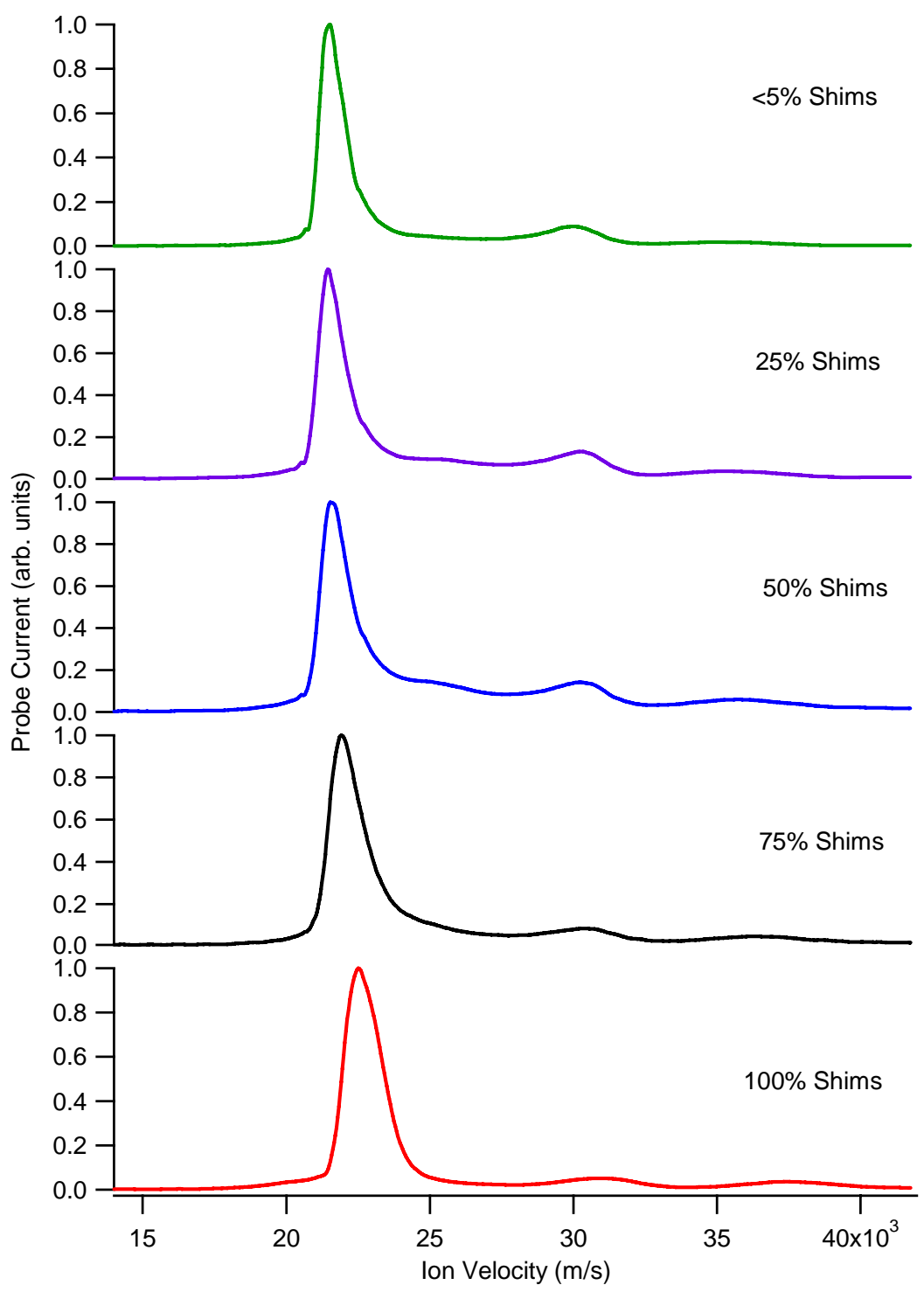

Figure 6-20: ExB probe traces taken as the level of shim current was varied. Data are for $400 \mathrm{~V}$ on the anode and $5 \mathrm{mg} / \mathrm{s}$ of xenon 


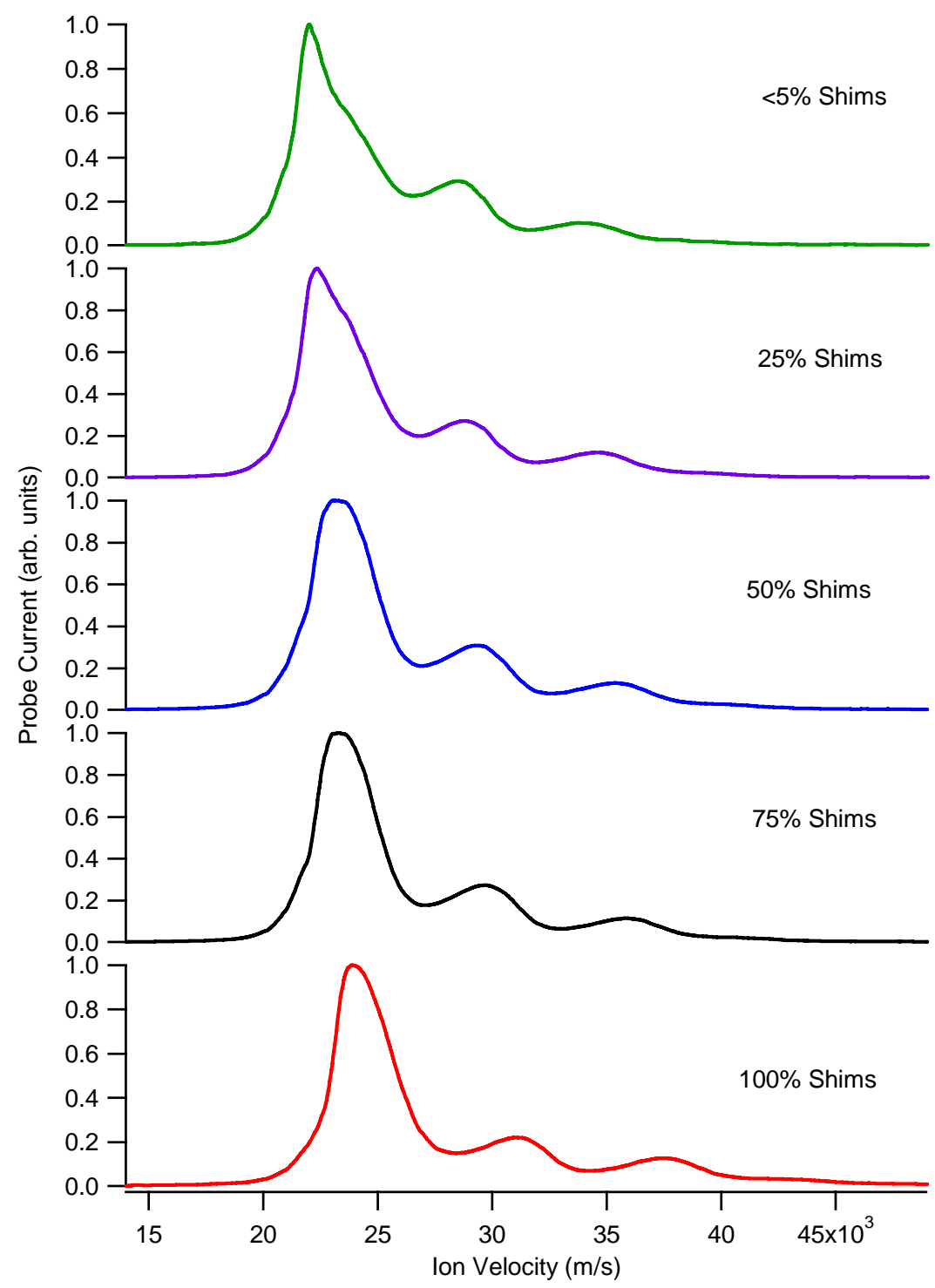

Figure 6-21: ExB probe traces taken as the level of shim current was varied. Data are for $400 \mathrm{~V}$ on the anode and $6 \mathrm{mg} / \mathrm{s}$ of xenon 


\section{Chapter 7: Determination of the Source of Observed Thruster Performance Changes}

\subsection{Performance Changes}

The segmented-electrode thruster showed some performance changes as discharge electron heating was shifted from the shims to the main anode. Thrust and specific impulse both increased with increasing shim voltage, indicating that the shim voltage is one of the factors that determine the total acceleration voltage experienced by the beam ions. Some hypotheses can be made about the accelerating potential and location of ionization from these results. Since the most probable ion energy was only dictated by the shims when the shim voltage was higher than the anode voltage, the highest voltage in the thruster must also affect the accelerating potential. Ionization could be happening either upstream or downstream of the shims. If the ionization is taking place upstream of the shims, there is a potential issue where an ion is created and can be accelerated towards the anode instead of out of the thruster. This could happen if ionization is taking place at the peak of the thruster potential. Ions could then fall through the potential created toward or away from the main anode. This backward acceleration would cause a large efficiency loss, as ions created and accelerated towards the anode would cost energy to create and accelerate, but provide no thrust. No large decrease in efficiency was seen, so backward acceleration of ions must not be happening in any significant quantity. Thus if ionization is taking place upstream of the shims, the potential hill inside the thruster must be such that the decrease to anode potential is very close to the anode itself. Ions thus would not be created where they could fall back towards the anode and would be accelerated out of the thruster. As shim voltage is decreased, the effect on the potential inside the discharge channel is reduced and the potential will become more dictated by the anode. Thus when the shim voltage is lower than the anode voltage, ions created upstream of the shims would be accelerated by the anode potential, not the shim potential, and show the ion energy behavior seen.

Ionization could also be taking place downstream of the shims. In this case, there is little potential for backward acceleration of ions at high shim voltages. This is because ions downstream of the shims are not at the crest of a potential hill, rather partway down a slope. Thus the newly created ions could not force 
their way past the shims to strike the anode, rather they would be accelerated out of the thruster. This creates a complication, however, in that the shim voltage was not the primary determining factor in ion energy at low shim voltages. If ionization is taking place downstream of the shims, then the accelerating potential downstream of the shims will be affected less by the shims and more by the main anode as shim voltage is lowered. When shim voltage was lower than the anode, then, the shims would have little to no effect on the accelerating potential. This indicates that the electron current impacting the shims has a large effect on potential, and thus as less current is diverted to the shims the accelerating potential will be affected more and more by the main anode.

The absence of a statistically significant change in efficiency as discharge electron heating was shifted from the shims to the main anode is important to future development of a shim electrode thruster. In a bismuth thruster, the discharge current will need to be constantly adjusted to maintain the anode at the proper temperature. If the efficiency was significantly lower at any of these levels of discharge current sharing, the power needs of the thruster will increase, thus increasing the size and mass of the power supply, making such a thruster system less desirable over chemical or gaseous EP systems. A change in efficiency was not seen, so the conclusion can be made that a bismuth thruster of the design described in Chapter 2 could be operated at a controlled propellant mass flow without a detrimental effect to the thruster efficiency.

A final conclusion which can be made from the performance measurements is that there is no backwards acceleration of propellant ions when the shims are at a higher voltage than the main anode. This was a concern due to the fact that when the shim voltage was higher than the anode voltage, it should have been possible for ions to be created upstream of the shims and accelerated back towards the anode. Backward acceleration can manifest in two ways, none of which were present to a significant degree in the performance measurements. First of these is a reduction in anode current due to positive ions canceling out some of the current from the negatively charged electrons impacting the anode. In the majority of thruster operating points, the current did not decrease as the shim voltage was raised above the anode voltage, rather in most cases the discharge current increased with shim voltage. The second manifestation of backward acceleration is in the performance of the thruster, especially efficiency. Any ions accelerated backwards will cost the thruster energy, both in ionization and the energy used to accelerate the ions. This will manifest as a direct efficiency loss to the thruster, as well as a reduction in thrust and specific impulse. It is 
unlikely that any significant quantity of ions are being accelerated back towards the anode when the shim voltage exceeds the main anode voltage since the thruster showed higher thrust and specific impulse at high shim voltages, and efficiency did not change.

\subsection{Plume Changes}

Each of the three plasma diagnostics performed assisted in determination of the source of the performance change seen as the thruster discharge current was shifted from the main anode to the shims. Through the use of each of these diagnostic methods, a separate aspect of the thruster plume was investigated, and could be correlated with the observed performance changes.

The results of these plasma diagnostics showed that the plume changes in two significant ways as discharge current is shifted from the main anode to the shims. When all of the thruster current was attached to the shims through high shim voltage, the thruster plume was narrower and consisted of higher-energy ions. This change was seen in the plume divergence data from the Faraday probe, which showed that the thruster plume exhibited a roughly $10 \%$ lower beam divergence at high shim voltage than at low shim voltage. This reduced plume divergence should have caused an efficiency increase, as the ions accelerated closer to the thruster axis will provide more benefit (thrust) than ions accelerated perpendicular to the thrust axis to a significant degree. This expected efficiency increase was not seen, however. The efficiency of the thruster did not significantly change as the discharge current was shifted from the main anode to the shims, thus the results from the plume divergence study and the thruster performance study are in conflict. Despite the statistical calculations which say that thruster efficiency did not change significantly, the efficiencies plotted in Figure 3-12 show slight increases with shim voltage. While the increases in efficiency with shim voltage were statistically insignificant, the possibility remains that the increase was real and simply overwhelmed by the error. This conflict will require future experimentation to resolve. Duplication of the performance measurements taken here will reduce the estimated variance, and may allow for resolution of a small change in thruster efficiency with shim voltage. Plasma diagnostics inside the thruster discharge channel may also provide insight into the nature of the discharge as the shim voltage is adjusted and may explain the lack of significant efficiency changes with plume divergence. 
Characterization of the ion energy showed that the ion acceleration is directly dependant on the shim voltage. Both the most probable and average ion energies were high for high shim voltage and low for low shim voltage. While the average ion energy decreased with respect to ground as the shim voltage was reduced the most probable ion energy did not. The most probable energy seemed to exhibit a large dependence on shim voltage only when the shim voltage was higher than the main anode voltage. When the shims were at a lower voltage than the main anode, the most probable ion energy remained roughly constant, and did not appear to be dependant on the shim voltage. This change in behavior indicates that the most probable ion energy is dependant on the highest voltage present in the thruster.

Ion energy has a small impact on thruster efficiency. As shown in the analysis in Appendix A, the fraction of thruster power required to ionize the propellant decreases as specific impulse increases. An increase in specific impulse (and thus ion energy) will cause a corresponding increase in efficiency. Both the average and most probable ion energies changed by approximately $10 \%$ over the range of shim voltage adjustments, which will reduce the ionization penalty to the thruster by roughly $10 \%$. Thus the efficiency of the thruster should have been higher at high shim voltages than it was at low shim voltages. This agrees with the conclusions seen from the plume divergence characterization, that the statistically insignificant efficiency changes seen as shim voltage was increased may be real, and will require additional experimentation to establish. The ion energy characterization will benefit greatly from plasma diagnostics inside the thruster discharge channel, as interior diagnostics may help determine why the ion energy changed.

Determination of the ion multiply charged fraction showed that there is little change in multiply charged ions as discharge current is shifted from the main anode to the shims. None of the ion fractions measured exhibited a statistically significant change as the thruster current was shifted from the main anode to the shims. While the extremely large error on these measurements complicates the determination of the multiply charged ion fraction somewhat, the lack of a change is in good agreement with the calculated thruster efficiency. An increased production rate of multiply charged ions will decrease thruster efficiency, thus the absence of a change as discharge current is beneficial to bismuth thruster operation. In this way, the multiply charged ion fractions correlate well with the observed efficiency of the thruster, in that neither changed as discharge current was shifted from the main anode to the shims. 


\subsection{Efficiency Analysis}

The anode efficiency calculated from the performance measurements does not show the increase with shim voltage expected from the plume diagnostics. Both the ion energy and plume divergence diagnostics showed changes with shim voltage which should have affected the anode efficiency. The difference between the expected versus observed changes in efficiency can be calculated from the equation:

$$
\eta_{A}=\eta_{B} \eta_{\varepsilon} \eta_{i} \eta_{\dot{m}} \frac{I_{i}}{I_{d}}
$$

As the anode efficiency is defined here as the product of several separate efficiencies, each component can be calculated separately and combined to determine how much the efficiency should have changed due to the plume measurements. Of importance to this calculation are the beam efficiency and acceleration efficiency, $\eta_{\mathrm{B}}$ and $\eta_{\varepsilon}$. The other factors are based on parameters which were not directly measured, and thus cannot be calculated. These parameters will thus be assumed equal to one for the purposes of this analysis. Beam efficiency is a measure of collimation in the exhaust plume. It is calculated by: ${ }^{6,38}$

$$
\eta_{B}=\frac{\int \cos ^{2}(\theta) j(\theta) d \theta}{\int j(\theta) d \theta} .
$$

The acceleration efficiency is the ratio of ion energy per unit charge to discharge voltage:

$$
\eta_{\varepsilon}=\frac{\bar{E}}{q V_{d}} .
$$

Acceleration efficiency is thus calculated from voltage and the average energy per unit charge determined by the RPA. The results of the Faraday probe sweeps provide the $j$ required for the beam efficiency. The discharge voltage $V_{d}$ used for these calculations will be the highest voltage present in the thruster. This was done because it provides an upper limit to the acceleration efficiency.

The efficiency analysis was performed on the data taken for $350 \mathrm{~V}$ on the anode and $5 \mathrm{mg} / \mathrm{s}$ of xenon. When all of the discharge current was on the shims, the beam efficiency was calculated to be $93 \%$. With all of the discharge current on the main anode, the efficiency was reduced to $92 \%$. The acceleration efficiency also decreased as shim voltage was lowered. The acceleration efficiency when all discharge current was on the shims was $92 \%$, and the efficiency with all current on the main anode was $90 \%$. The 
product of these two efficiencies will provide an estimate of how much the thruster efficiency should have changed when discharge current was shifted from the shims to the main anode. The approximate efficiency with all current on the shims is $86 \%$, while the efficiency with all current on the main anode was $83 \%$.

This would indicate that the anode efficiency with all of the discharge current on the anode should be $96.5 \%$ of the efficiency with all current on the shims.

Use of the calculated anode efficiency in this calculation is complicated by the large error in the measurement. None of the changes in anode efficiency were determined to be statistically significant. For the purposes of this analysis, then, the confidence interval will be ignored, and the calculated values used. The efficiency calculated from the thrust measurements showed a decrease from approximately 53\% to $52 \%$ anode efficiency with the same change in shim and anode current. Thus the efficiency of the thruster with all current on the anode was approximately $98 \%$ of that when all current was on the shims. This means that the calculated change in anode efficiency was roughly half that expected by the plume divergence and ion energy. When the confidence interval on the anode efficiency is added to these calculations, the potential reduction in efficiency as the discharge current is shifted from the shims to the main anode could feasibly be equal to or larger than the change predicted by the probe diagnostics.

Several potential processes could cause the change in anode efficiency to be smaller than that predicted by the plume diagnostics. Most likely of these are increased electron mobility and a possible backward flow of ions at high shim voltages. Electron mobility is a measure of how many electrons are able to escape the magnetic field in the thruster and strike the anode. It is a significant source of efficiency loss as each electron that strikes the anode must be re-emitted by the thruster cathode, thus costing power. Ion backflow is the possible acceleration of ions towards the anode when the shims are at a high voltage. Significant amounts of ion backflow would be disastrous for thruster operation, as these ions would still cost energy to ionize, but provide no thrust and cause erosion of the anode face. Ion backflow, if present, should only affect the thruster when the shim voltage was higher than the anode voltage. For other operating conditions, with shim voltage below anode voltage, ions should not be accelerated towards the anode. 


\section{Chapter 8: Conclusions}

Development of a Hall thruster that operates on condensible propellant requires a method for delivering propellant vapor to the discharge channel of the thruster. This is a significant change from gaspropelled thrusters that require no such system. A method has been developed which uses the main anode of the thruster as a reservoir and evaporator for propellant, and thus can inject the propellant directly into the thruster discharge. The heat energy obtained from thruster operation, which is normally radiated into space, can be harnessed to provide the necessary anode temperature for condensible propellant evaporation. This prevents an unfavorable positive feedback loop condition, however, in that an evaporation rate slightly higher than desired will increase the thruster power, increasing heating on the anode, which will in turn increase the evaporation rate of propellant. Modification of the thruster to a segmented electrode design may be an enabling innovation, allowing direct thermal control of the anode. Addition of shim electrodes in the Hall thruster discharge channel allows control of the discharge current attachment, and hence, the discharge current heating. This diversion of current also diverts the discharge electron heating, which accounts for roughly $10 \%$ of the total discharge power of the thruster. This diversion of discharge electron heating allows for direct regulation of the anode temperature by removing a significant portion of the heat flux into the anode. Development of such a thruster as a future flight technology requires characterization of the effect the addition of segmented electrodes has on performance.

To characterize the effect adjustable segmented anodes have on the performance of a Hall thruster, a thruster was constructed based on an Aerojet BPT-2000 thruster. The original electromagnet and ceramic structures were copied directly from the original thruster, and the single main anode and propellant distributor was replaced with a smaller anode and shim electrodes separated by ceramic spacers. The new main anode also functioned as a propellant distributor. The thruster was operated with xenon propellant. This allowed the anode temperature to be decoupled from mass flow, enabling accurate mass flows to be provided to the thruster. The thruster was operated with several performance and plasma diagnostics to determine the nature of the performance changes and to attempt to determine the source of the changes. 


\subsection{Thruster Performance}

The first diagnostic employed was to measure the performance of the thruster. The measurement of thrust under this experimental paradigm showed that the performance of the thruster is in fact altered by the diversion of discharge current from the shims to the main anode. While a change in thruster performance with the addition of segmented electrodes has been seen before, ${ }^{17,18,19}$ previous experimental efforts did not focus on using the segmented anodes for thermal control. The segmented anode control mechanism used in the experiments reported here represents a new approach to thruster operation.

The thruster showed statistically significant changes in both thrust and specific impulse as a function of shim voltage, while thruster anode efficiency did not change within the limits of the experimental error. Both the thrust and specific impulse increased with shim voltage. This was not an entirely unexpected result; high voltages accelerate propellant ions to high velocities, thus increasing both thrust and specific impulse. The dependence on shim voltage indicates that both the main anode shim electrode voltages were driving factors in the ion exit velocity.

In an eventual bismuth evaporating scheme, discharge current must be shifted from the main anode to the shims on a constant basis to maintain or adjust the main anode temperature; thus the thrust and specific impulse will be variant over the course of a mission. The changes in thrust and specific impulse are small relative to the absolute measurements, however, at a less than $10 \%$ reduction in either measurement between the maximum and minimums for a given anode voltage and mass flow configuration.

Thruster efficiency did not change as a function of the discharge current on the shims. This result is extremely important to future development of a segmented anode thruster as power requirements, and thus power supply size, will not change as discharge current is shifted from the shims to the main anode. This means that a segmented electrode thruster can divert current from the shims to the main anode as necessary to control the evaporation rate of a condensible propellant without negatively impacting the efficiency of the thruster.

The performance measurements thus showed that a segmented electrode Hall thruster is feasible for future development as a flight technology. Overall, the changes to thrust and specific impulse with shim voltage were small and do not present a significant challenge to implementation on a spacecraft. The 
lack of a statistically significant change in thruster efficiency means that a thruster can be operated at all levels of shim voltage without requiring an increase in power to provide a given thrust.

\subsection{Plume Diagnostics}

Three different plasma diagnostics were utilized to attempt to determine where the changes in thruster performance come from. Thruster plume divergence, ion energy, and ion multiply charged fraction were measured, and each contributed to determining the nature of the thruster discharge and the source of the performance changes.

The thruster plume divergence was reduced by roughly $10 \%$ when the discharge current was moved from the main anode to the shims. This means that the thruster is effectively accelerating the propellant more efficiently, and less energy is being wasted accelerating propellant ions perpendicular to the thruster axis. This reduction in plume divergence can account for the increased thrust and specific impulse of the thruster. The reduced plume divergence is in disagreement with the efficiency measurements, however. The reduced plume divergence should manifest as an increase in efficiency due to less acceleration power being wasted on off-axis acceleration.

The ion energy diagnostics showed that average and most probable ion energy were dependant on the shim voltage. The average ion energy decreased with shim voltage over the entire range of shim voltage adjustment, while the most probable energy showed a large decrease as current was initially shifted away from the shims, then remained relatively constant over the rest of the adjustment of discharge current back to the main anode. This change occurred over the range in which the shim voltage was higher than the main anode voltage, thus indicating that the bulk of the ions saw an accelerating potential which was dictated by the highest voltage in the thruster. At operating points where the shims were at low voltages relative to the anode, the effective acceleration was dictated by the anode and thus relatively independent of the shim voltage. At high shim voltages, the energy increased rapidly, indicating that the dominating factor in the size of the accelerating potential became the shim voltage. Thus the highest voltage present in the thruster is the dominant factor in the acceleration of the bulk of the propellant ions. Coupling these measurements with measurements inside the thruster discharge channel may assist in determination of the nature of the potential hill and the effect the shim electrodes have on the plasma inside the thruster. 
Combination of the ion energy and plume divergence measurements yielded an estimate for the expected change in thruster efficiency. The thruster should have shown an approximate $4 \%$ multiplicative decrease in efficiency as the discharge current was shifted from the main anode to the shims. The thruster showed an approximately $2 \%$ multiplicative decrease in efficiency, however, indicating that there are other sources of thruster efficiency which were not characterized by this study. It is also possible that the large experimental error on the thruster efficiency measurements overwhelmed a real change in efficiency. Acquiring duplicate data to that analyzed here will greatly assist in reducing the width of the confidence interval on efficiency, and determine if the change in efficiency predicted by the plume diagnostics is present in the thruster.

Measurement of the multiply charged ion fraction showed no significant change as a function of the location of discharge current. Additionally, calculation of the average ion charge from these data showed the same was true, that the average charge is unaffected by the location of discharge current attachment. This conclusion means that a thruster operating with a condensible propellant, using the segmented anodes for thermal control, will not produce multiply charged ions at a variable rate, and thus that the thruster can be operated at any level of discharge current sharing without an increase in the detrimental effects of multiply charged ions. The error of the probe and charge fraction measurements were very high, however, complicating the analysis of the charge fractions. While some error is to be expected when measuring the extremely small currents in the ExB probe, the resolution of the probe served to complicate analysis. Development of a model for the ions in the thruster discharge plasma that can predict and describe the data acquired by the $\mathrm{ExB}$ probe will greatly improve the accuracy of the probe as a plasma diagnostic for Hall thrusters.

There are three primary ways to improve the resolution of an ExB probe beyond that seen in the experiments reported here. First is to reduce the orifice sizes in the collimator and filter of the probe. As with any spectrometric device, the smaller the orifice through which the signal to be measured can pass, the better the resolution. The maximum distance an off-axis ion can travel, is directly calculated from the orifice sizes. Thus ions with large off-axis angles and/or positions will not be able to pass through small orifices, reducing the resolution error of the measurement. Orifice size reduction may cause signal strength issues, however, as fewer ions will be able to enter the probe and be filtered. Smaller orifices also present 
an alignment problem, as all three orifices must be collinear to allow ions with the tuned velocity to pass directly through unimpeded. Furthermore, orifices of much smaller sizes than those used in the experiments reported here present a manufacturing and maintenance problem; small orifices can become easily occluded with sputtered debris. This is not a large problem in a xenon thruster, however it could potentially be a large problem in a bismuth thruster, as the condensible propellant could collect on the orifice plates and occlude the orifices.

A second method to improve the resolution of the probe is addition of a drift tube at the exit of the filter. ${ }^{35}$ The tube would act as a collimator at the exit of the probe, requiring that ions not only be in the proper position to pass through the filter exit orifice, but also have the correct velocity so that they can pass through the drift tube exit. This is a much more stringent requirement on ion detection than simply being able to pass the filter exit orifice, and thus will filter out many of the ions which have velocities greater or less than the tuned velocity, but still are deflected in such a way that they pass through the filter exit orifice. This method would also lead to a smaller signal than that seen in the experiments reported here, however it should not affect the ions at the tuned velocity and thus will provide smaller but much more resolved signals.

A potential third method involves increasing the magnetic field strength in the probe. The velocity measurement error is directly proportional to the magnetic field strength in the probe. Magnetic fields of high strength cause very high forces on charged particles passing through them, which requires a much higher electric field to compensate for. A small change in ion velocity at these high magnetic field strengths will cause a much higher change in force than at low strengths, and thus a small change in ion velocity will cause the ion to deflect too much to pass through the exit orifice of the filter. Increasing the magnetic field strength is problematic, however. The rare earth magnets already in the probe are the strongest available, preventing any upgrade of field strength from another magnet material. Use of better ferromagnetic materials in the construction of the probe may aid in keeping the magnetic field contained within the probe, but the increase would be small. In order to significantly increase magnetic field strength, a series of electromagnets could be used. This solution has its own problems, however. The magnetic field produced by an electromagnet is highly dependant on temperature, so the probe must be either very closely temperature controlled or monitored so that the magnetic field is known at all times. Addition of a 
powerful water- or nitrogen-cooled electromagnet system, however, would allow nearly unlimited magnetic field strength. Application of $1 \mathrm{~T}$ in the filter of the probe would reduce the energy resolution error by approximately a factor of three. Such a system would require a complete redesign of the probe casing, however, as the magnetic stainless steel used is not sufficient for such high field strengths and will saturate too easily to be useful. Operation of such a probe would require extremely high electric field strengths, and thus very high voltages on the electrode plates. In the probe utilized for the experiments reported here, it required approximately $100 \mathrm{~V}$ on the electrode plates to tune the probe to the most probable ion velocity for singly charged ions. If the magnetic field strength was raised to $1 \mathrm{~T}$, it would require almost $300 \mathrm{~V}$ to achieve the same tuning. A final problem is the physical size of such a system. The probe utilized in the experiments reported here had a mass of roughly $20 \mathrm{~kg}$ with the water-cooling shell. Addition of a large electromagnet system would further increase this mass and may present vacuum facility integration issues, especially on a motion table.

\subsection{Future Work}

The experiments and conclusions reported here would benefit significantly from the following additional work:

- Duplication of thrust, ion energy, and ion multiple charge fraction measurements. The primary advantage of duplicate measurements is an increase in statistical accuracy of the analysis. A secondary advantage to duplicate measurements is that the interactions between experimental factors can be resolved.

- Redesign of the ExB probe to increase accuracy. This could include any of three methods, all of which will reduce the signal seen by the probe and could complicate ion current measurements due to the low signal. The easiest of these to implement would be addition of a drift tube to the end of the ExB filter, however this would require a completely new analysis of the resolution of the probe, while the other methods would only require application of the same analysis to a different set of constants. Development of a theoretical model which can determine the ion charge fractions from the ExB probe 
data could also greatly increase accuracy, and would allow the probe to be used for much more information than currently available.

- Finally, the same set of experiments can be performed on a bismuth thruster using the same general design as the xenon thruster employed here. The data and conclusions reported here would greatly aid the characterization of a bismuth thruster and provide significant insight into the differences in performance between bismuth and xenon 


\section{Appendix A: Motivation for Condensible Propellants}

An emerging hurdle in high-power Hall thruster development has been the use of xenon as the propellant of choice. While xenon has several advantages as a propellant, namely low ionization energy, high atomic mass and easy storage and flow metering, several disadvantages preclude the use of xenon in very high power thrusters. The first disadvantage to xenon as an EP propellant is its high cost. Currently xenon can be purchased for approximately $\$ 6.65$ per standard liter $(\$ 1140 / \mathrm{kg})$ in small quantities. ${ }^{39}$ Using current commercial prices, a 500-kW Hall thruster operating at 60\% anode efficiency and 2,000 s Specific Impulse ( $\mathrm{I}_{\mathrm{sp}}$ ) will consume $\$ 6,400$ of xenon per hour of operation. These costs can be extrapolated to \$153,600 per test day, and \$64M for a 10,000-hour mission. Longer-duration missions utilizing larger thrusters or many smaller thrusters can quickly become relatively expensive to supply with propellant. To reduce this cost, more economical propellants need to be utilized. Studies have focused on krypton as a more-economical alternative to xenon, while maintaining the general design of a gas-propelled thruster. ${ }^{40}$

The second major disadvantage to xenon is in ground testing. Thruster exhaust must be evacuated from a test facility in order to maintain a space-like vacuum. Typically, this is accomplished with cryogenic vacuum pumps. High facility pressures will affect the thruster performance and beam characteristics through an increase in charge-exchange ions created by collisions between exhaust ions and neutral atoms that remain the vacuum chamber. For a $500-\mathrm{kW}$ thruster operating at 2,000 s $\mathrm{I}_{\mathrm{sp}}$ and $60 \%$ efficiency, $1.6 \mathrm{~g} / \mathrm{s}$ of xenon will enter the chamber. In order to maintain a pressure of $1 \times 10^{-5}$ Torr $\left(6.7 \times 10^{-4}\right.$ Pa) of xenon, the facility vacuum pumps must be capable of nearly 20M liters-per-second of pumping throughput. The pressure chosen was previously determined by Randolph to be sufficient to nearly eliminate facility backpressure effects. More recent observations ${ }^{41}$ have shown this not to be entirely accurate, however for lack of another estimate of facility pressure, the estimate of Randolph will be used. At a cost of roughly $\$ 1$ per-l/s, ${ }^{42}$ this translates to roughly $\$ 20 \mathrm{M}$ in pumping equipment. The pumping requirements scale linearly with thruster power, so a 1-MW thruster will require nearly $\$ 40 \mathrm{M}$ in pumping equipment. Additional costs include the large vacuum chamber, support infrastructure and recurring costs such as liquid nitrogen. Unlike propellant costs, facility costs cannot be reduced unless gaseous propellant usage is eliminated, as any gaseous propellant will require evacuation from the facility. 
Condensible propellants, defined as those species existing in either solid or liquid state at Standard Temperature and Pressure $\left(0{ }^{\circ} \mathrm{C}\right.$, one atmosphere pressure) (STP) offer significant advantages for facility cost. While xenon, krypton, and other gaseous propellants must be actively evacuated from the test chamber, condensible propellants will naturally condense on the chamber walls, requiring no pumping. Thus, condensible propellants are 'self-pumping,' requiring only enough vacuum pump capacity to reach and maintain high vacuum with little to no gas load. Condensible propellants may also cost less than xenon or krypton, reducing testing costs further. Vacuum facility contamination may be an issue with condensable propellants, however. The coating of interior surfaces with propellant may require special handling procedures, especially for toxic and reactive propellants.

\section{A.1 Analysis of alternative Propellants}

Many factors contribute to the suitability of a Hall thruster propellant. Factors that affect performance include ionization energy, atomic mass, and ease of mass flow system construction. Condensible propellants offer a challenge in mass flow systems, as traditional gas-fed systems utilized on xenon and krypton thrusters cannot be used. Generally, the higher the melting and boiling points of a propellant, the harder it will be to design and fabricate a mass flow system. Propellant ionization is another source of inefficiency. As energy spent on ionization is not available for acceleration, reduction of ionization energy will directly increase the efficiency of a thruster. Several practical issues also contribute to the suitability of a candidate propellant. These include cost, toxicity/reactivity, and potential for spacecraft contamination. Reducing propellant cost is of obvious benefit, as less-expensive propellants allow for reduced testing and mission costs. Several alternative propellant options have been

considered. ${ }^{43,44,45,46,47,48}$ Some physical properties of these propellants, including xenon as a point of comparison, are in Table A-1. This section will discuss the relative merits and difficulties with the many propellant alternatives.

Several of the Hall thruster propellants considered are toxic and/or reactive. These include cadmium, ${ }^{49}$ cesium, ${ }^{50}$ iodine, ${ }^{51}$ and mercury. ${ }^{52}$ While each of these propellants offer lower propellant costs and ionization energy than xenon, they all present problems in a testing environment. 
Table A-1: properties of several Hall thruster propellant alternatives

\begin{tabular}{|c|c|c|c|c|c|}
\hline Propellant & $\begin{array}{c}\text { Melting } \\
\text { Point }\left({ }^{\circ} \mathrm{C}\right)\end{array}$ & $\begin{array}{c}\text { Boiling } \\
\text { Point }\left({ }^{\circ} \mathrm{C}\right)\end{array}$ & $\begin{array}{c}\text { First Ionization } \\
\text { Energy }(\mathrm{eV})\end{array}$ & $\begin{array}{c}\text { Atomic Mass } \\
(\mathrm{amu})\end{array}$ & Cost Per Kg \\
\hline Bismuth (Bi) & 271 & 1559 & 7.3 & 209.0 & $\$ 6^{53}$ \\
\hline Cadmium (Cd) & 321 & 765 & 9.0 & 112.4 & $\$ 25^{54}$ \\
\hline Cesium (Cs) & 29 & 685 & 3.9 & 132.9 & $\$ 40,000^{55}$ \\
\hline Iodine (I) & 113 & 182 & 10.4 & 126.9 & $\$ 484^{56}$ \\
\hline Krypton (Kr) & -157 & -153 & 14.0 & 83.8 & $\$ 295^{57}$ \\
\hline Mercury (Hg) & -39 & 357 & 10.4 & 200.6 & $\$ 4^{58}$ \\
\hline Xenon (Xe) & -112 & -108 & 12.1 & 131.3 & $\$ 1138$ \\
\hline
\end{tabular}

Of these, iodine and cesium present significant reactivity hazards, while each presents a significant chronic and/or acute toxicity hazard. Currently there are no active development efforts on these propellants.

Three of the propellants considered are relatively non-toxic and non-reactive. Xenon and krypton present essentially no toxicity threat except through displacement of oxygen ${ }^{59,60}$ Reactivity is also of little concern, as krypton will not spontaneously react with any substance, and xenon will react only with fluorine. Bismuth presents little hazard. It is considered mildly toxic and safety precautions involve exposure limitation only. ${ }^{61}$ While conversion of a Hall thruster from xenon to krypton requires trivial redesign of the mass flow system, bismuth presents significant development issues due to its condensable nature. Bismuth thruster development is under consideration at Stanford/Jet Propulsion Laboratory (JPL) under the Very High Impulse Thruster with an Anode Layer (VHITAL) program, ${ }^{62}$ Busek, and Michigan Technological University. ${ }^{63}$

\section{A.2 Energetics of propellant alternatives}

\section{A.2.1 Acceleration Kinetics}

The most obvious disparity between propellant performance characteristics is a change in thrust under identical discharge voltage due to the different atomic mass. The mass ratio of the propellants is defined as:

$$
M R=\frac{M_{n}}{M_{X e}}
$$


where $M R$ is the mass ratio of the propellant to xenon. The thrust produced by a thruster is defined as $F=u_{e} \dot{m}$. Assuming that ions of any species are accelerated through the same voltage, the exhaust velocity will be:

$$
u_{e}=\sqrt{\frac{2 q_{i} V_{a}}{M_{x e}}} \sqrt{\frac{1}{M R}}=\left(u_{e}\right)_{X e} \sqrt{\frac{1}{M R}}
$$

and the thrust force is:

$$
F=\left(u_{e}\right)_{X e} \dot{m} \sqrt{\frac{1}{M R}}
$$

which is the thrust force of a xenon thruster divided by the square root of the mass ratio. Mass flows may either be the same between different propellants, or they may be adjusted so the same number of moles of propellant are utilized per second. In the case where mass flow is constant, $\dot{m}$ will be the same for all propellant options. In the case where molar flow is constant, the mass flow will change according to MR, while the discharge current will remain approximately constant. If the mass ratio is adjusted so molar flow rate and thus thruster current is constant, then the thrust will be the thrust of a xenon thruster multiplied by the square root of the mass ratio. The trends in thrust for different propellant ion masses are plotted in Figure A-1. $I_{\mathrm{sp}}$ under constant discharge voltage will be modified similarly to exhaust velocity. As $\mathrm{I}_{\mathrm{sp}}$ is given by the equation:

$$
I_{s p}=\frac{u_{e}}{g}
$$

$I_{s p}$ for any propellant is equal to the value for a xenon thruster, divided by the square root of the mass ratio. So a thruster that would normally operate on xenon at 2,000 s $\mathrm{I}_{\mathrm{sp}}$ would operate on krypton at 2,500 s, and on bismuth at 1,583 s for the same discharge voltage, with the other propellant options falling between the krypton and bismuth specific impulses. In order to maintain the same $\mathrm{I}_{\mathrm{sp}}$ with different propellants, the discharge voltage would need to be modified. Large variations in the discharge voltage 


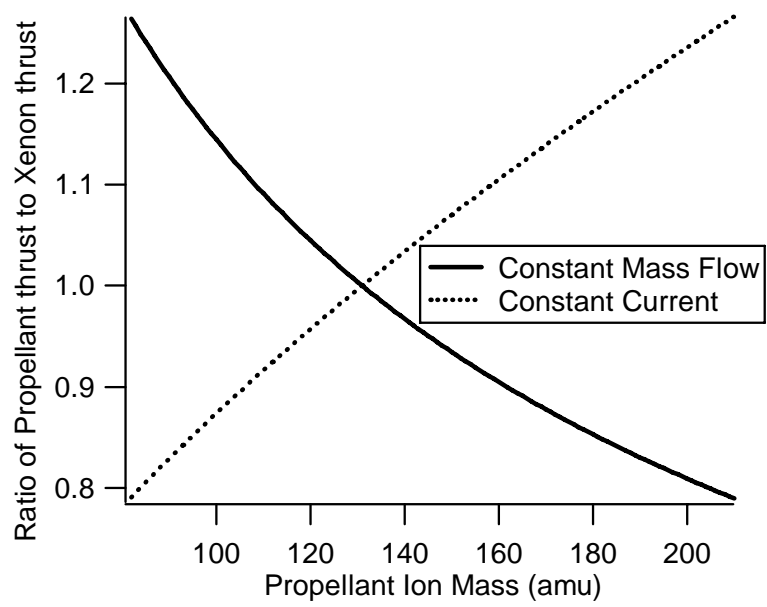

Figure A-1: Change in thrust as a function of propellant ion mass, assuming constant acceleration voltage

(outside the current SOA of 200-600V) cause complications in plasma acceleration physics that may preclude the use of heavy atoms at high $\mathrm{I}_{\mathrm{sp}}$, or light atoms at low $\mathrm{I}_{\mathrm{sp}}$.

Aside from the exhaust kinetics, the choice of propellant has a direct influence on thruster efficiency. Ionization production represents a fundamental source of inefficiency, since any energy spent in creating an ion from a neutral is not available for conversion to beam kinetic energy. As the ionization energy is exhibited as a direct power loss from the thruster, keeping the ionization energy at a minimum will increase thruster efficiency. While ionization energy is typically expressed as the energy required to singly ionize one neutral atom (eV/atom), a more convenient form for thruster analysis is the amount of energy required per-kilogram of mass flow. This factor, $E_{i o n}$, then accounts for the difference in ionization potentials as well as the difference in atomic masses of the candidate species, and can be calculated by dividing the ionization energy by the mass of an atom. $\mathrm{E}_{\mathrm{ion}}$ can then be employed in the equation:

$$
P_{\text {ion }}=\dot{m} E_{\text {ion }} \text {. }
$$

We can use $P_{\text {ion }}$ to calculate the maximum theoretical efficiency of an acceleration process assuming that the only energy loss is propellant ionization. Expressing the kinetic power in the exhaust beam as

$$
P_{k i n}=\frac{1}{2} \dot{m} u_{e}^{2}=\frac{\dot{m} g^{2} I_{s p}^{2}}{2} .
$$

Combining Eqn (A-5) with Eqn (A-6) yields: 


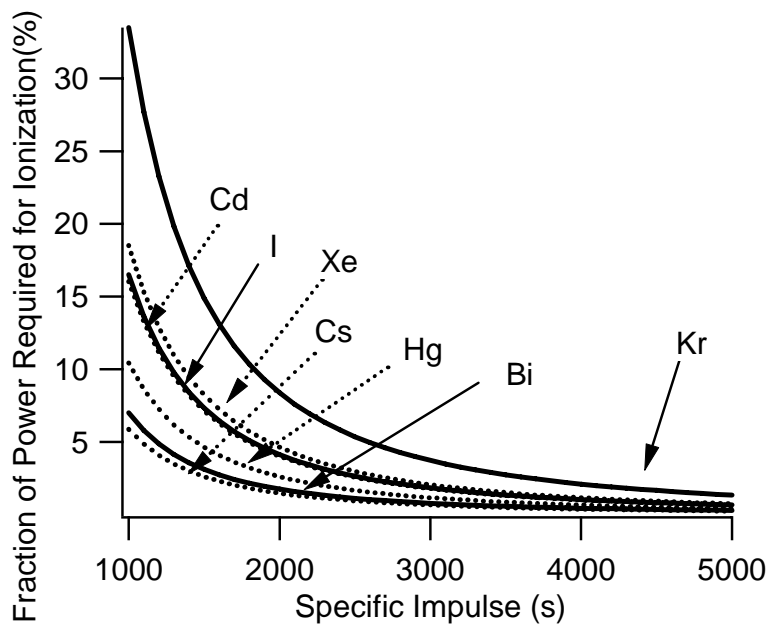

Figure A-2: Fraction of thruster power required for ionization vs. $\mathbf{I}_{\mathrm{sp}}$ for all propellants examined

$$
\frac{P_{i o n}}{P_{k i n}}=\frac{2 E_{i o n}}{g^{2} I_{s p}^{2}} .
$$

Eqn A-7 is then the theoretical minimum efficiency penalty required to singly ionize and accelerate the propellant as a function of $\mathrm{I}_{\mathrm{sp}}$. These ratios are plotted in Figure A-2.

The propellants with lower ionization energy-per-mass $\left(E_{\text {ion }}\right)$ require a smaller fraction of the total thruster power to ionize the propellant. It is also interesting to note that the difference between propellants becomes nearly insignificant at high $\mathrm{I}_{\mathrm{sp}}$. At 1,000 s, the fractions range from approximately $6 \%$ for cesium up to $34 \%$ for krypton, while at 5,000 s no propellant option requires significantly more than one percent of total thruster power (krypton is highest at 1.3\%). It should be noted that these calculations are a theoretical minimum where all of the propellant is ionized, there are no doubly-charged ions, no energy is spent promoting atoms or ions to excited states, and all of the ions are produced only once; there is no wall recombination and re-ionization. Any re-ionization process will present an energy loss as an ion is essentially being created twice, requiring twice the energy expenditure. The ionization power will differ in a real thruster, however the relative scaling between propellants should remain similar.

\section{A.2.2 Collision Considerations}

Any neutral propellant that is not ionized within the discharge chamber prior to escaping the thruster internal volume represents inefficiency in propellant utilization (it is not electrostatically accelerated and contributes negligibly to thrust). The probability of ionization for a given atom subject to 
an electron collision is given by the ionization cross section. These cross sections are dependent on the energy of the impacting (ionizing) electron and the atomic structure of the propellant atom. Experimentally determined ionization cross-sections for bismuth ${ }^{64}$, cesium ${ }^{65,66}$, iodine ${ }^{67}$, krypton $^{68}$, mercury ${ }^{69}$, and xenon were available in literature. Cross-section measurements for cadmium could not be found. A comparison of xenon with the other propellants is plotted in Figure A-3.

In general, the lower the atom's ionization energy the larger the ionization cross section. Thus cesium shows itself to be the most amenable to ionization, due to the extremely low ionization energy. However, bismuth and mercury do not have significantly lower cross sections than cesium. The smallest cross sections calculated were for the two propellants currently in use; xenon and krypton. Thus any change from xenon or krypton to another propellant discussed here will result in an increased probability of ionization within the discharge chamber.

Using the ionization cross-section, the rate of ionizing collisions can be calculated. The rate of ionizing collisions is given by: ${ }^{70}$

$$
v=\bar{\sigma}_{e} \sqrt{\bar{v}_{n}^{2}+\bar{v}_{e}^{2}} \text {. }
$$

In order to calculate estimates of $v$ the electron velocity distribution function was calculated based on an

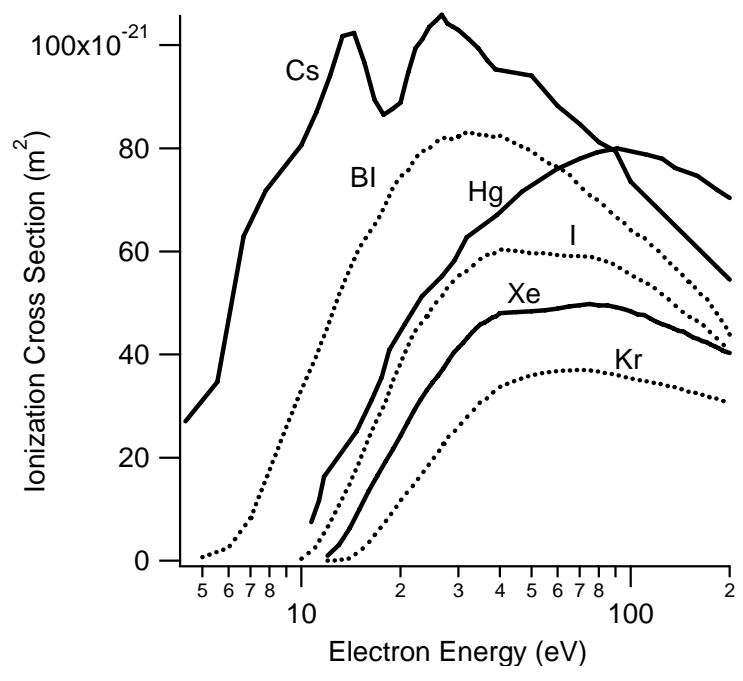

Figure A-3: Electron-Impact Ionization Cross Sections for bismuth, cesium, iodine, krypton, mercury, and xenon between threshold and $200 \mathrm{eV}$ 
assumed $20 \mathrm{eV}$ temperature and the Maxwellian distribution. ${ }^{71}$ The average electron-impact ionization cross section was calculated by integrating the product of the electron energy distribution function and the ionization cross section. $\bar{\sigma}$ is estimated assuming the propellant atoms are emitted with a thermal velocity distribution in equilibrium with the anode/diffuser temperature. Average electron velocity is estimated similarly, assuming the temperature of the electrons is $20 \mathrm{eV}$. Given typical anode temperatures of $700{ }^{\circ} \mathrm{C}$, the neutral velocity will be in the range of $300-500 \mathrm{~m} / \mathrm{s}$ for all species. Since the average electron velocity is four orders of magnitude higher, the neutral velocity can be neglected. Thus, collision frequencies will scale simply with ionization cross-section and electron density. An electron number density of $2 \times 10^{18} \mathrm{~m}^{-3}$ was measured by Haas and Gallimore in Ref. Error! Bookmark not defined. and is used here as a representative value to calculate collision frequencies that may be typical within a Hall thruster discharge. Of critical importance to determination of the propellant utilization of a thruster is the electron temperature in the discharge channel. As it is impossible to determine the exact effect switching to another propellant will have on the electron temperature, values of electron temperature measured in xenon thrusters are used here for calculations. Calculated values of the collision frequency are in Table 2.

While the trends in collision frequency among the propellant candidates simply mirror the cross section scaling of Figure 3, the magnitude of $v$ becomes particularly important in calculating the propellant utilization efficiency. In order to measurably contribute to the thrust, a propellant atom must be ionized before it is permitted to escape the discharge chamber. Thus if the residence time is defined as $t_{r}=L / v_{n}$ and $v_{n}$ is defined as:

$$
\bar{v}_{n}=\sqrt{\frac{k T}{2 \pi m}}
$$

The factor $v_{r}$ gives the ratio of residence time to collision time. Physically, this factor represents the average number of ionizing collisions experienced by a propellant atom before diffusing out of a discharge chamber of length $L$. If this factor is low, it is probable that propellant atoms will escape the discharge without being ionized. Using the calculated collision frequencies, assuming neutral atoms leave the gas diffuser with a temperature of $700^{\circ} \mathrm{C}$, and assuming the neutral atoms must travel $100 \mathrm{~mm}$ to leave the channel, the average number of ionizing collisions for each propellant species is shown in Table A-2. 
Table A-2: Number of ionizing collisions experienced by propellant atoms in a 100-mm channel.

\begin{tabular}{|c|c|c|c|}
\hline Propellant & $\begin{array}{c}\text { Collision Frequency } \mathbf{( x 1 0} \\
\mathbf{H z}\end{array}$ & $\begin{array}{c}\text { Neutral Diffusion Velocity } \\
(\mathbf{m} / \mathbf{s})\end{array}$ & Number of Collisions \\
\hline Bismuth & 4.1 & 161 & 26 \\
\hline Cesium & 5.5 & 202 & 32 \\
\hline Iodine & 2.7 & 207 & 18 \\
\hline Krypton & 1.4 & 254 & 20 \\
\hline Mercury & 3.3 & 165 & 10 \\
\hline Xenon & 2.1 & 203 & \\
\hline
\end{tabular}

While the gross simplification used in determining the number of collisions precludes any confidence in the absolute magnitude of the numbers, the relative trends between propellants is a reliable indicator of utilization. The calculated ionizing collision rates show significant differences between species. When compounded with the neutral diffusion velocity, some propellants show a much higher utilization than others. Krypton suffers here, as it has the lowest ionization cross section and the highest neutral diffusion velocity. Cesium, as expected, will experience the most ionizing collisions due to its very high ionization cross section. Of particular interest here, however, is bismuth, with an estimated number of collisions only slightly less than cesium. This is due in large part to the large cross section and extremely low neutral diffusion velocity of bismuth.

While it is important to have an electron population with enough energy to ionize the propellant, it is equally important not to have an electron population so energetic as to produce significant amounts of multiply-charged ions. Previous studies on xenon thrusters have given maximum electron temperatures above $15 \mathrm{eV} .^{72,73}$ Probe-based studies of a 5-kW thruster at several operating points show the temperature is dependent on flow rate, and can be nearly $30 \mathrm{eV}$ in low mass-flow conditions. ${ }^{\text {Error! Bookmark not defined. }}$ Higher mass flows appear to cool the electrons, as the drifting electrons cannot acquire as much energy through 'falling' towards the thruster anode after a collision. Any population of electrons with temperature greater than the second ionization potential of the propellant may create multiply ionized propellant ions. Multiply charged ions represent an inefficient use of propulsive power. For instance, a doubly charged ion will contribute twice the discharge current (and hence, draw twice the power) as a singly charged ion, however will only be accelerated to a velocity $\sqrt{2}$ times more than its single counterpart. 
Table A-3: First and Second Ionization Energies

\begin{tabular}{|c|c|c|}
\hline Propellant & First Ionization Energy (eV) & Second Ionization Energy (eV) \\
\hline Bismuth & 7.3 & 16.7 \\
\hline Cadmium & 9.0 & 16.9 \\
\hline Cesium & 3.9 & 23.2 \\
\hline Iodine & 10.4 & 19.1 \\
\hline Krypton & 14.0 & 24.4 \\
\hline Mercury & 10.4 & 18.8 \\
\hline Xenon & 12.1 & 21.2 \\
\hline
\end{tabular}

Formation of multiply charged ions may be an issue for some propellants as shown in Table A-3. Xenon has the drawback of a high first ionization energy, but its second ionization energy is quite high (21.2 eV); thus it does not readily produce $\mathrm{Xe}^{2+}$ in typical Hall thruster plasmas, with approximately $90 \%$ of the xenon ions singly charged. ${ }^{74}$ Cesium and krypton also will not form large fractions of multiple ions. Other elements, such as bismuth and cadmium, may be sensitive to multiply charged ion efficiency losses in an electron population with temperatures similar to those seen in xenon devices.

\section{A.3 Analysis of spacecraft interactions for bismuth, xenon, and krypton}

At this point, bismuth, xenon, and krypton will be chosen for further analysis. As bismuth is nearly as efficient as cesium, without the toxicity or reactivity drawbacks, it appears to be the best choice for a highthrust, low-Isp thruster. Krypton is chosen as an alternative only for high-Isp operation, as it is too energetically costly to ionize to be useful in a low-Isp thruster.

The sputter rate of a propellant is highly important to thruster lifetime, especially at high Isp when propellant ions have significant energy. Typically, the maximum lifetime of the thruster is determined by the time required for sputter erosion to wear through the discharge channel walls and start eroding the electromagnet poles. The sputter yield of ions normally incident on a solid surface can be calculated by: ${ }^{75}$

$$
Y(E)=0.42 \frac{\alpha_{s} Q_{s} S_{n}(E)}{U_{0}\left[1+0.35 U_{0} S_{e}(\varepsilon)\right]}\left[1-\sqrt{\frac{E_{t h}}{E}}\right]^{2.8}
$$

Of particular interest is $E_{t h}$, as sputtering does not occur when incident ions have energies below $E_{t h}$. The sputtering threshold energy is calculated by: 


$$
E_{t h}=\left(\frac{4}{3}\right) \frac{U_{0}}{\gamma}
$$

where $\gamma$ is the elastic energy transfer factor. This factor is involved in the calculation of the threshold sputtering energy, and thus affects the sputtering rate at all energies. $\gamma$ is calculated by the equation:

$$
\gamma=\frac{4 M_{i} M_{s}}{\left(M_{i}+M_{s}\right)^{2}} .
$$

Calculations were made for bismuth, krypton, and xenon ions incident on a carbon surface as a representative thruster material (it is unclear whether or how this model can be applied to BN as a target material). While carbon is not normally used in Hall thrusters, there has been experimental effort into using it as an electrode material. ${ }^{76,77}$

The sputtering threshold for ions on carbon was found to be different for each species; $202 \mathrm{eV}$ for bismuth, $135 \mathrm{eV}$ for xenon, and $95 \mathrm{eV}$ for krypton. These results mean that for a given distribution of ion energies, fewer bismuth ions will be above the sputtering threshold than xenon or krypton. For ions above threshold, the model can be used to predict sputter rates. The calculated sputter rates over a range of ion energies are plotted in Figure A-4.

The results of sputtering calculations show that bismuth will induce less sputtering on carbon substrates at energies anticipated in Hall thruster operation. At high energies (greater than 1,200 eV) bismuth will cause higher sputtering than xenon and krypton, however this range is beyond the operational

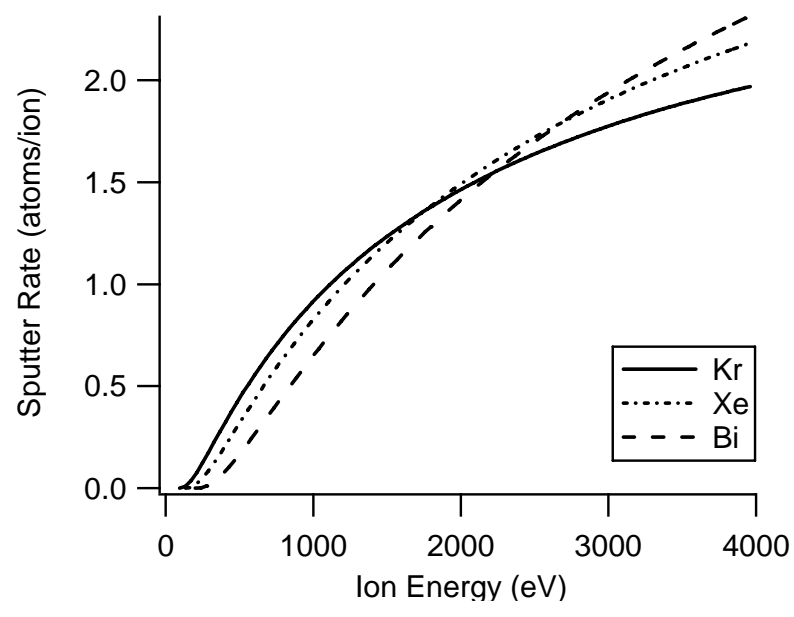

Figure A-4: Sputter Yield of carbon under bismuth, xenon and krypton ion collisions 
envelope of SOA Hall thrusters. Bismuth’s erosion advantage is further extended if sputtering is analyzed under constant mass flow. As bismuth has an atomic mass of $209 \mathrm{amu}$, nearly $60 \%$ greater than the atomic mass of xenon (131 amu) and 2.5 times as high as krypton (84 amu), a given mass flow of bismuth will contain fewer ions. To determine the effect of the increased mass of bismuth, the calculations were scaled to indicate the mass of surface sputtered away per unit mass of ions. These results are displayed in Figure A-5 and show that bismuth will cause less erosion than xenon or krypton given equal mass flows. These results are encouraging for the lifetime of a bismuth thruster, as the thruster structures should exhibit lower erosion for the same total mass throughput. The erosion rate for bismuth remains less than xenon or krypton until the ion energies approach 10,000 eV, at which point the erosion rates converge. For ion energies higher than 10,000 eV, which are not included in Figure 5, the lighter propellants become favorable. It should be noted that the erosion rate is primarily driven by the ion mass and atomic number, and the ratios of these to the atoms in the surface. As the other propellants analyzed fall between krypton and bismuth in size, they will exhibit sputter rates somewhere between those of krypton and bismuth.

One complicating factor for use of bismuth or any other condensable propellant is deposition on spacecraft surfaces. As the surfaces of a spacecraft may be well below the melting point of a condensable propellant, ions impacting on spacecraft surfaces will stick similarly to the interior of a vacuum tank. This would present a problem for solar arrays and other optical systems, as it is not desirable to have coatings of opaque metals on the transparent lenses and plates of such systems. Other spacecraft integration issues include coating of radiator and dielectric surfaces. Coatings of propellant atoms on radiators will reduce

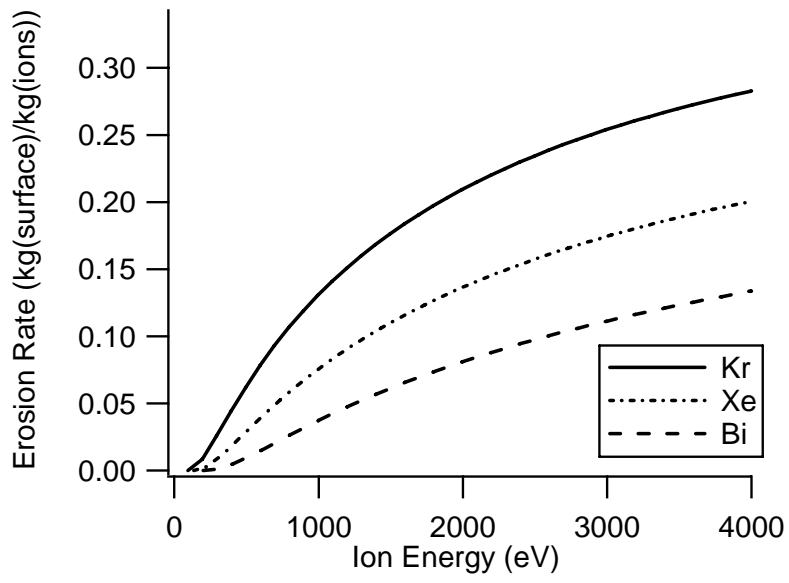

Figure A-5: Erosion Rate of Carbon per kilogram of bismuth, xenon, and krypton ions 
emissivity and radiative efficiency. Dielectrics are very vulnerable to propellant deposition, as conductive coatings on the surface of a dielectric will render it ineffective. Spacecraft design and interaction studies will require future research.

\section{A.4 Feed System for Condensible Propellant Operation}

Development of a feed system for a condensible propellant is a major technical obstacle. A large advantage to mercury, as used in NASA thrusters, was that it was a liquid at room temperature; it could be transported through propellant lines with little heat input and the only significant need for external heating was for the evaporator. ${ }^{78}$ This system may not be practical for bismuth or the other condensible propellants analyzed here, as the entire propellant feed system would need to be maintained above the melting point of the propellant. This would not present much of a problem for cesium due to the very low melting point, but for bismuth operation, the propellant must be maintained in excess of $271^{\circ} \mathrm{C}$. Bismuth thrusters in Soviet research utilized a propellant feed system that was maintained in excess of $1,000^{\circ} \mathrm{C}$, in order to flow gaseous bismuth directly into the thruster. Additionally, the thruster body was heated above $1000^{\circ} \mathrm{C}$ using resistive heaters. This method may present a problem, however, in that a number of heaters consuming significant amounts of power are likely required. While using large amounts of power for a propellant evaporation system is possible in ground testing, any development of a flight system cannot allow for such

expenditures. The use of condensible metal propellants in future Hall thrusters will require development of an energy-efficient feed system. An additional energy expenditure is the evaporation of the propellant, requiring enough heat input to overcome the heat of fusion of the propellant. The energy required to overcome the heat of fusion of the propellant is negligible compared to the total thruster power; Bismuth, for example, requires $54 \mathrm{~mW}$ of power per $\mathrm{mg} / \mathrm{s}$ of mass flow to evaporate.

\section{A.5 Summary of analysis of propellant options}

For high- $\mathrm{I}_{\mathrm{sp}}$ missions, krypton may prove advantageous as it provides the highest $\mathrm{I}_{\mathrm{sp}}$ of the options presented here for a given acceleration voltage, and at such high exhaust energies the large ionization cost is minimized. The erosion rate due to sputtering will also be lower for krypton than other propellants at very high energies (above 10,000 eV). For high-thrust, low- $\mathrm{I}_{\mathrm{sp}}$ missions, however, the large heavy atoms 
may provide a benefit. Bismuth provides higher thrust per unit mass than the lighter propellant options given equal accelerating voltages and is very easy to ionize. While cesium is the easiest to ionize, once the average number of collisions in a Hall thruster channel is analyzed, it proves to be only incrementally better than bismuth. Cesium is also only marginally better in the fraction of thruster power required for ionization than bismuth. Heavy atoms also provide an advantage in erosion rate for most ion energies, up to extremely high specific impulses.

Condensible propellants also offer significant advantages over gases. Primary of these is the elimination of the costly and complex pumping apparatus required to maintain acceptable vacuum levels. This benefit is complementary to the increased efficiency of condensable species for low-Isp, and correspondingly high flow rate, missions that are most expensive to ground test. Condensible propellants may cause spacecraft contamination issues, as unlike the gaseous propellants condensibles will deposit on spacecraft surfaces.

Among the condensible propellant options, bismuth shows the most promise for Hall thruster use and is likely superior to other candidates for high-thrust, low-Isp missions. It combines the advantages of higher thrust at the same discharge current as a xenon thruster, low cost, ease of ionization, and lower sputter erosion rate than the other propellants examined. The main disadvantage to bismuth, however, is that any thruster design must incorporate a method of heating the bismuth evaporator to temperatures where evaporation is significant. It is also much less likely that spacecraft surfaces will be warm enough to avoid or reduce bismuth deposition due to the high melting and boiling points. A second, and possibly significant, disadvantage of bismuth may be its propensity to form doubly charged ions at lower electron temperatures than xenon. 


\section{Appendix B: Experimental and Statistical Analysis Methods}

In order to obtain a thorough characterization of the performance and plume changes as shim voltage is adjusted to move discharge current from the shims to the anode, data were taken at several thruster operating points. The operating points examined consisted of a full factorial design consisting of three factors. The first two factors, main anode voltage and propellant mass flow, had three levels each (main anode voltages of 300, 350, and $400 \mathrm{~V}$, and propellant mass flows of 4, 5, and $6 \mathrm{mg} / \mathrm{s}$ ). These were chosen as several of these operating conditions were near the original BPT-2000 anode voltages and power levels, while also providing a throttling range of approximately $800 \mathrm{~W}$ above and below the $2 \mathrm{~kW}$ design. The location of current deposition was the third factor in the experiments, and was split into five levels; $100 \%, 75 \%, 50 \%, 25 \%$, and $<5 \%$ of the discharge heating on the shims. Control of the shim voltage relative to the main anode voltage was the source of the changes in discharge heating levels, however the use of shim voltage as a factor in the experiment instead of the discharge heating could not be done, as the shim voltages required for a given level of discharge heating was not constant across different main anode voltages and propellant flow rates. As the experiment used different numbers of levels of each factor, a fractional design could not be used and the full factorial design of 45 thruster operating points was necessary. No duplicate measurements were performed. Determination of whether a factor caused a significant effect on any of the measurements performed was accomplished through the use of the analysis of variance method. ${ }^{79}$ This method uses all data collected to develop an estimate of the sample variance, and is dependent on the experimental factors being independent from each other and that the data are normally distributed. The requirement that all factors be independent is well-met in the experiments reported here; anode voltage, propellant mass flow, and the location of discharge electron heating were controlled separately from each other and thus one factor could be easily changed without any effect on the other two. The requirement that the data be normally distributed is difficult to determine from these data. Determination of whether the data are normally distributed can usually only be accomplished through many repetitions of the measurements, determining the distribution about the sample mean, and deciding whether 
the distribution is normal or otherwise. As there were no duplicate measurements taken, there can be no proof of a normal distribution of the measurements.

Two results can be obtained from the analysis of variance and the ANOVA table generated by the method; a highly accurate estimate of the variance, and the statistical probability that a given factor or combination of factors have a significant effect on a measured property. In the experiments reported here, no duplicate measurements were taken. As the use of duplicates is typically necessary to determine the experimental error, another method must be used to find the error estimate. In this case, the interactions between experimental factors were used to estimate the error. In any three-factor experiment, there are three two-factor interactions and one three-factor interaction; the interactions between factors A and B, between A and C, between B and C, and between A, B, and C. In this case, factor A was the anode voltage, factor B the mass flow, and factor $\mathrm{C}$ the amount of discharge electron heating on the shims. As previously stated, factor $\mathrm{C}$ was chosen based on having the independent variable of the data points equally spaced and in the same place for every combination of factors A and B; using shim voltage for C would not allow this, as it was not the same for all operating points. Analysis was done under the null hypothesis assumption; that none of the experimental factors had a significant effect on the property of interest (thrust, specific impulse, efficiency, beam divergence, ion energy, etcetera). Thus the P-value calculated in each table is the confidence that the hypothesis is true; large numbers mean the hypothesis is true, that the factor had no effect on the measurement, while small numbers mean that the factor had a significant effect. Typically P-values over 5\% indicate the factor had no statistically significant effect. This assumption is open to interpretation, however, and larger P-values may be chosen to invalidate the null hypothesis. An additional benefit of performing analysis of variance is it supplies an estimate of the sample variance. The mean squared error $\left(M S_{E}\right)$ produced by the analysis is a direct estimate of the sample variance, thus the square root of $M S_{E}$ is an estimate of the standard deviation of the sample. The tdistribution is used for calculation of the confidence interval. Under the normality assumption, a distribution of data will approach the perfect normal distribution as the number of data points goes to infinity. This is clearly not possible, so the t-distribution is used, which is a modification of the normal distribution to adjust for the increased uncertainty inherent with a small number of measurements. Calculation of the confidence interval, then, is accomplished by: 


$$
X=X_{m} \pm t_{1-\alpha, d f} \sqrt{\frac{M S_{E}}{d}} .
$$

Eqn. B-1

In the case of the experiments performed, the number of measurements at each factor combination is one, so the equation simplifies to:

$$
X=X_{m} \pm t_{1-\alpha, d f} \sqrt{M S_{E}} .
$$


${ }^{1}$ Jahn, R.G., Physics of Electric Propulsion, McGraw-Hill, 1968

${ }^{2}$ Martinez-Sanchez, M. and Pollard, J.E., “Spacecraft Electric Propulsion - an Overview”, Journal of Propulsion and Power, Vol. 14, No. 5, p. 688-693 1998

${ }^{3}$ Gulczinski III, F. S., Spores, R.A., "Analysis of Hall-effect Thrusters and Ion Engines for Orbit Transfer Missions,” 32nd Joint Propulsion Conference, Orlando, FL, July 1996, AIAA-96-2973

${ }^{4}$ Sutton, G.P., and Biblarz, O., Rocket Propulsion Elements, John Wiley and Sons, 2001, ISBN 0-471$32642-9$

${ }^{5}$ Bugrova, A.I., et. al., "Physical Processes and Characteristics of Stationary Plasma Thrusters with Closed Electrons Drift,” $22^{\text {nd }}$ International Electric Propulsion Converence, Viareggio, Italy, 14-17 October, 1991, Viareggio, Italy

${ }^{6}$ Kim, V., "Main Physical Features and Processes Determining the Performance of Stationary Plasma Thrusters,” Journal of Propulsion and Power Vol. 14, No. 5, p. 736, September-October 1998

${ }^{7}$ Choueiri, E.Y., "Fundamental Difference Between the Two Hall Thruster Variants," Physics of Plasmas Vol. 8, No. 11, p.5025, November 2001

${ }^{8}$ Pote, B., and Tedrake, R., "Performance of a High Specific Impulse Hall Thruster," $27^{\text {th }}$ International Electric Propulsion Conference, Pasadena, CA, 15-19 October, 2001, IEPC-2001-035

${ }^{9}$ Guerrini, G., Michaut, C., et. al., “An Intense Hall-Type Ion Source for Satellite Propulsion,” Review of Scientific Instruments Vol. 69, No. 2, p.804, February 1998

${ }^{10}$ Koppel, Christophe R., Marchandise, Frederic, et. al., "The SMART-1 Electric Propulsion Subsystem In Flight Experience,” 40 ${ }^{\text {th }}$ Joint Propulsion Conference, 11-14 July 2004, Fort Lauderdale, FL, AIAA-2004-3435

${ }^{11}$ Grishin, S.D., et. al., "Characteristics of a Two-Stage Ion Accelerator with an Anode Layer," Zhurnal Prikladnoi Mekhaniki I Technicheskoi Fiziki no. 2, pp. 28-36, March-April, 1978

${ }^{12}$ Tverdokhlebov, S., Semenkin, A., et. al., "Bismuth Propellant Option for Very High Power TAL thruster,”, 40th AIAA Aerospace Sciences Meeting, Reno, NV., AIAA-2002-0348 
${ }^{13}$ King, L.B., Michigan Technological University, Houghton, MI, U.S. Patent \#70591111, published $06 / 13 / 2006$

14 Massey, D.R., “Development of a Vaporizing Liquid Anode for Hall Thrusters,” 40 ${ }^{\text {th }}$ Joint Propulsion Conference and Exhibit, 11-14 July, 2004, Ft. Lauderdale, FL, AIAA-2004-3768

${ }^{15}$ Massey, D.R., King, L.B., et. al., “:Progress on the Development of a Direct Evaporation Bismuth Hall Thruster," 41 $1^{\text {st }}$ Joint Propulsion Conference and Exhibit, Tucson, AZ, 10-13 July 2005, AIAA-4232

${ }^{16}$ Kieckhafer, A.W., Massey, D.M., et. al., "Performance and Active Thermal Control of a Hall Thruster with Segmented Anodes,” Journal of Propulsion and Power, Accepted for publication 02/2007

17 Fisch, N.J., Raitses, Y., et. al., "Variable Operation of Hall Thruster with Multiple Segmented Electrodes,” Journal of Applied Physics Vol. 89, No. 4, p.2040, 15 February 2001

${ }^{18}$ Raitses, Y., Dorf, L.A., et. al., "Plume Reduction in Segmented Electrode Hall Thruster,” Journal of Applied Physics Vol. 88, No. 3, p. 1263, August 2000

19 Diamant, K.D., Pollard, J.E., et. al., “Investigation of a Segmented Electrode Hall Thruster,” $40^{\text {th }}$ Joint Propulsion Conference and Exhibit, Fort Lauderdale, FL, 11-14 July 2004, AIAA 2004-4098

${ }^{20}$ Kieckhafer, A.W., et. al., "Effect of Segmented Electrodes on the Beam Profile of a Hall Thruster,” $40^{\text {th }}$ Joint Propulsion Conference and Exhibit, Ft. Lauderdale FL, July 11-14, 2004, AIAA-2004-4101

21 King, D., Tilley, R., et. al., "Development of the BPT Family of US-Designed Hall Current Thrusters for Commercial LEO and GEO Applications, 34 ${ }^{\text {th }}$ Joint Propulsion Conference, July 12-15, 1998, Cleveland OH, AIAA-1998-3338

${ }^{22}$ Haag, T., "Design of a Thrust Stand for High Power Electric Propulsion Devices," $25^{\text {th }}$ Joint Propulsion Conference and Exhibit, July 10-13, 1989, Monterey, CA, AIAA-89-2829

${ }^{23}$ Hofer, R. R., "Development and Characterization of High-Efficiency, High-Specific Impulse Xenon Hall Thrusters," Ph.D. Dissertation, University of Michigan, 2004

${ }^{24}$ Domonkos, M. T., Marrese, C. M., et. al., "Very Near-Field Plume Investigation of the D55," 33 Joint Propulsion Conference, Seattle, WA, July 6-9, 1997. AIAA-97-3062

${ }^{25}$ Kusamoto, D., Mikami, K., and Komurasaki, K., “Exhaust Beam Profiles of Hall Thrusters,” 
${ }^{26}$ Hagstrum, H.D., “Auger Ejection of Electrons from Tungsten by Noble Gas Ions,” Physical Review vol. 96, no. 2, p.325, October 15, 1954

${ }^{27}$ Hutchinson, I.H. Principles of Plasma Diagnostics, Cambridge University Press, 2002, ISBN 0521803896

${ }^{28}$ Absalamov, S.K., et. al., "Measurement of Plasma Parameters in the Stationary Plasma Thruster (SPT-100) Plume and its Effect on Spacecraft Components," 28 ${ }^{\text {th }}$ Joint Propulsion Conference, 6-8 July, 1992, Nashville, TN, AIAA-1992-3156

${ }^{29}$ Garkusha, V., et. al., "Ion Energy Measurement of a D-55 Hall Thruster," $3^{\text {rd }}$ International Conference of Spacecraft Propulsion, 10-13 October, 2000, Cannes, p. 359

${ }^{30}$ Gombosi, T., Gaskinetic Theory, Cambridge University Press, 1994 ISBN 0-521-43966-3

${ }^{31}$ Marrese, C. M., Haas, J. M., Domonkos, M. T., Gallimore, A. D., Tverdoklebov, S., and Garner, C., “D-100 Performance and Plume Characteristics on Krypton,” 32nd Joint Propulsion Conference, Orlando, FL, July, 1996. AIAA-96-2969

${ }^{32}$ Kemp, R.F., and Sellen, J.M., "Plasma Potential Measurements by Electron Emissive Probes," Review of Scientific Instruments vol. 37, no. 4, pp. 455-461, 1966.

${ }^{33}$ Kim, S-W, and Gallimore, A.D., "Plume Study of a 1.35-kW SPT-100 Using an ExB Probe,” AIAA$99-2423$

${ }^{34}$ Lieberman, M.A., and Lichtenberg, A.J., Principles of Plasma Discharges and Materials Processing, Wiley-Interscience, 1994, ISBN 0-471-00577-0

${ }^{35}$ Kim, S. W., Experimental Investigations of Plasma Parameters and Species-Dependent Ion Energy Distribution in the Plasma Exhaust Plume of a Hall Thruster, Ph.D. Dissertation, University of Michigan, 1999

${ }^{36}$ King, L. B., Transport-property and Mass Spectral Measurements in the Plasma Exhaust Plume of a Hall-effect Space Propulsion System, Ph.D. Dissertation, University of Michigan, 1998

${ }^{37}$ Manzella, D.H., “Stationary Plasma Thruster Plume Emissions,” IEPC-93-097, Sept. 1993

${ }^{38}$ Ross, J.L., et. al., "Efficiency Analysis of a Low Discharge Voltage Hall Thruster,” Journal of Propulsion and Power, submitted 06/2007 
${ }^{39}$ Praxair, Inc. Verbal quotation, 6/2005, www.praxair.com

${ }^{40}$ Kim, V., Popov, G., et. al., "Investigation of SPT Performance and Particularities of it’s Operation with $\mathrm{Kr}$ and $\mathrm{Kr} / \mathrm{Xe}$ Mixtures,” 27th International Electric Propulsion Conference, Pasadena, CA, October, 1997. IEPC-01-065

${ }^{41}$ Walker, M.R., "Effects of Facility Backpressure on the Performance and Plume of a Hall Thruster," Ph.D. Dissertation, University of Michigan, 2004.

${ }^{42}$ PHPK Inc. Verbal Quotation for TM-1200 model cryopump, 6/2005

${ }^{43}$ Grishin, S.D., et. al., "Characteristics of a Two-Stage Ion Accelerator with an Anode Layer," Zhurnal Prikladnoi Mekhaniki I Tekhnicheskoi Fiziki 2, p 28-36, March-April 1978

${ }^{44}$ Tverdokhlebov, O.S., and Semenkin, A.V., "Iodine Propellant for Electric Propulsion - To Be Or Not To Be,” 37th AIAA/ASME/SAE/ASEE Joint Propulsion Conference, Salt Lake City, UT, July, 2001, AIAA 2001-3350

45 Bugrova, A.I., Kim, V., et. al., "Physical Processes and Characteristics of Stationary Plasma Thrusters with Closed Electrons Drift,” 22nd International Electric Propulsion Conference, Viareggio, Italy, 1991, IEPC-91-079

${ }^{46}$ Dressler, R.A., et. al., "Propellant Alternatives for Ion and Hall Effect Thrusters," 38th AIAA Aerospace Sciences Meeting, AIAA 2000-0602

${ }^{47}$ Kerslake, W.R., and Ignaczak, L.R., "Development and Flight History of SERT II Spacecraft," Journal of Spacecraft and Rockets 30, p.258, 1993

${ }^{48}$ Sovey, J.S., Rawlin, V. K, and Patterson, M.J., “A Synopsis of Ion Propulsion Development Projects in the United States: SERT I to Deep Space I,” AIAA 99-2270, NASA TM-1999-209439

${ }^{49}$ See, for example, http://www.osha.gov/SLTC/cadmium/, accessed 6/2005

${ }^{50}$ See, for example, http://www.espi-metals.com/msds's/cesium.htm, accessed 6/2005

${ }^{51}$ See, for example, http://www.jtbaker.com/msds/englishhtml/i2680.htm, accessed 6/2005

${ }^{52}$ See, for example, http://www.jtbaker.com/msds/englishhtml/M1599.htm, accessed 6/2005

${ }^{53}$ See, for example, http://minerals.usgs.gov/minerals/pubs/commodity/bismuth/bismumyb03.pdf

${ }^{54}$ See, for example, minerals.usgs.gov/minerals/ pubs/commodity/cadmium/cadmmyb02.pdf 
${ }^{55}$ Catalog price \$198/5g 99.999\% purity, from Sigma-Aldrich, Inc., www.sigmaaldrich.com

${ }^{56}$ Catalog price \$242/500g USP, from Sigma-Aldrich, Inc., www.sigmaaldrich.com

${ }^{57}$ Praxair, Inc. Verbal quotation, 6/2005, www.praxair.com

${ }^{58}$ See, for example, http://minerals.usgs.gov/minerals/pubs/commodity/mercury/430798.pdf, accessed $6 / 2005$

${ }^{59}$ See, for example, http://www.vngas.com/pdf/g54.pdf, accessed 6/2005

${ }^{60}$ See, for example, http://www.scottecatalog.com/msds.nsf/0/86e8c8fec163c40985256a0a004e2f4c?OpenDocument, accessed $6 / 2005$

${ }^{61}$ See, for example, http://www.sciencestuff.com/msds/C1309.html, accessed 6/2005

${ }^{62}$ Tverdokhlebov, S., Semenkin, A., and Polk, J., “Bismuth Propellant Option for Very High Power TAL Thruster,” 40th AIAA Aerospace Sciences Meeting, AIAA 2002-0348

${ }^{63}$ Massey, D.R, King, L.B., and Makela, J.M., "Progress on the Development of a Direct Evaporation Bismuth Hall Thruster,” $41^{\text {st }}$ Joint Propulsion Conference, 10-13 July 2005, Tucson, AZ, AIAA 2005-4232

${ }^{64}$ Freund, R.S., et. al., “Cross-Section Measurements for Electron-Impact Ionization of Atoms,” Phys. Rev. A 41(7), p. 3575, 1 April 1990 (APS)

${ }^{65}$ Nygaard, K.J., “Electron-Impact Ionization Cross Section in Cesium,” J. Chem. Phys. 49(5), p.1995, 1 September 1968 (AIP)

${ }^{66}$ McFarland, R.H., and Kinney, J.D., “Absolute Cross Sections of Lithium and Other Alkali Metal Atoms for Ionization by Electrons,” Phys. Rev. 137(4A), p.A1058, 15 February 1965 (APS)

${ }^{67}$ Hayes, T.R., et. al., “Absolute Electron-Impact-Ionization Cross-Section Measurements of the Halogen Atoms,” Phys. Rev. A. 35(2) p. 578, 15 January 1987 (APS)

${ }^{68}$ Wetzel, R.C., et. al., “Absolute Cross Sections for Electron-Impact Ionization of the Rare Gas Atoms by the Fast-Neutral-Beam Method,” Phys. Rev. A. 35(2) p.559, 15 January 1987 (APS)

${ }^{69}$ Kieffer, L.J., and Dunn, G.H., “Electron Impact Ionization Cross-Section Data for Atoms, Atomic Ions, and Diatomic Molecules: I. Experimental Data,” Rev. Mod. Phys. 38(1) p.1, January 1966 (APS)

${ }^{70}$ Gombosi, T.I., Gaskinetic Theory, Cambridge University Press, 1994, ISBN 0-521-43966-3 
${ }^{71}$ Haas, J.M., and Gallimore, A.D., "Considerations on the Role of the Hall Current in a LaboratoryModel Thruster,” 37th AIAA/ASME/SAE/ASEE Joint Propulsion Conference, Salt Lake City, UT, July 2001, AIAA 2001-3507

${ }^{72}$ Meezan, N. B., Hargus, W.A., and Cappelli, M.A., “Anomalous Electron Mobility in a Coaxial Hall Discharge Plasma,” Phys. Rev. E. 63, 026410

${ }^{73}$ Bishaev, A.M., and KIM, V., "Local Plasma Properties in a Hall-Current Accelerator with an Extended Acceleration Zone,” Sov. Phys. Tech. Phys. 23(9), p.1055

${ }^{74}$ King, L.B., Transport-property and Mass Spectral Measurements in the Plasma Exhaust Plume of a Hall-effect Space Propulsion System, Ph.D. Dissertation, University of Michigan, 1998.

${ }^{75}$ Nastasi, Michael, Mayer, James W., and Hirvonen, James K., Ion-Solid Interactions Fundamentals and Applications, Cambridge University Press, 1996, ISBN 0-521-37376-X

${ }^{76}$ Raitses, Y. and Fisch, N.J., "Parametric Investigations of a Nonconventional Hall Thruster,” Phys. Plasmas 8(5), p. 2579, May 2001 (AIP)

${ }^{77}$ Raitses, Y., Keidar, M., et. al., “Effects of Segmented Electrode in Hall Current Plasma Thrusters,” J. Appl. Phys. 92(9), p.4906, 1 November 2002 (AIP)

${ }^{78}$ Kerslake, William R., "Design and Test of Porous-Tungsten mercury Vaporizers," 9th AIAA Electric Propulsion Conference (AIAA 72-484), Bethesda, MD April 17-19, 1972

${ }^{79}$ Montgomery, D.C., Design and Analysis of Experiments, John Wiley and Sons 2001, ISBN 0-471$31649-0$ 